\title{
Neurovestibular analysis and falls in Parkinson's disease and atypical parkinsonism
}

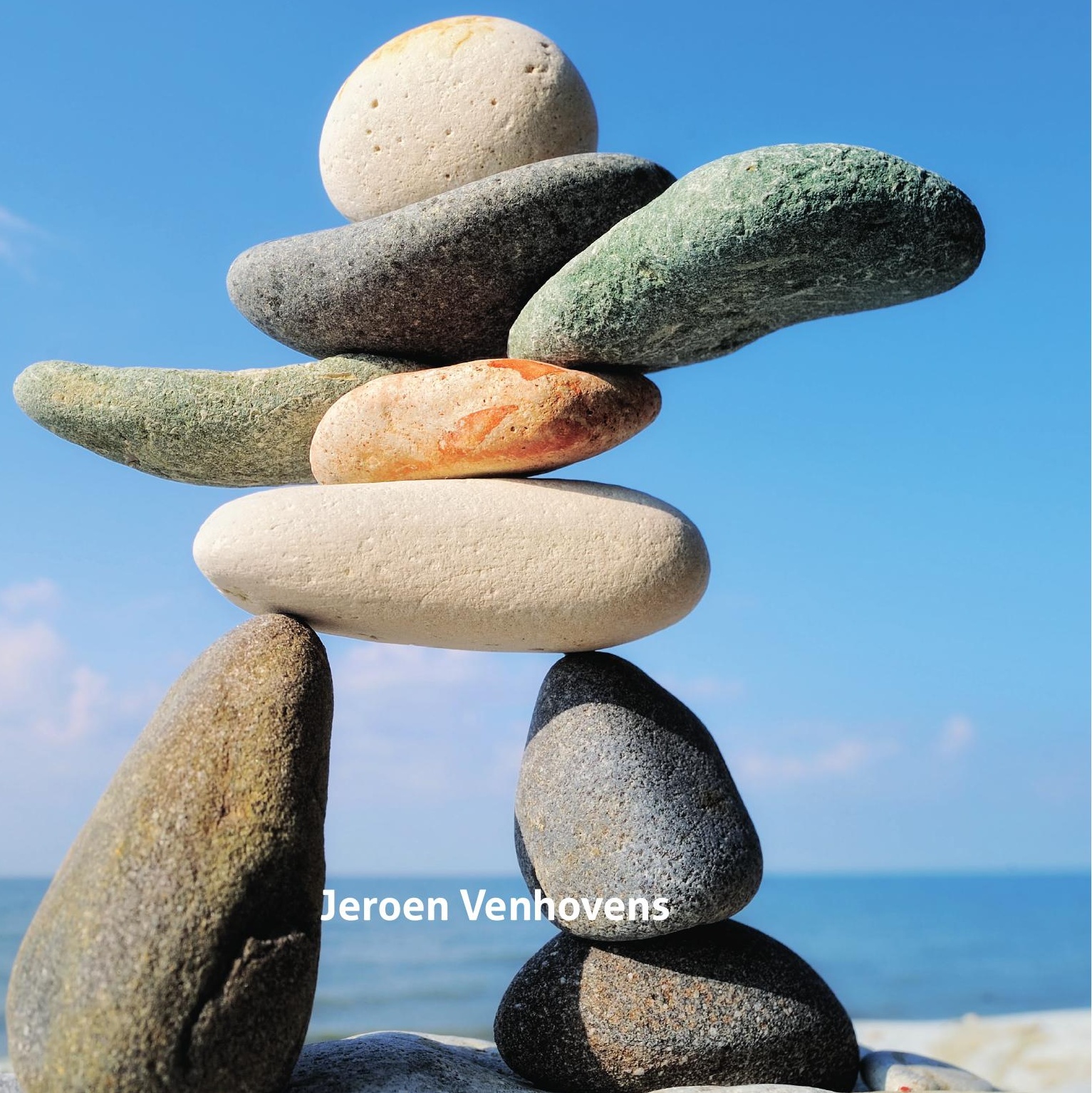





\section{Neurovestibular analysis and falls in Parkinson's disease and atypical parkinsonism}


Neurovestibular analysis and falls in Parkinson's disease and atypical parkinsonism (C) Jeroen Venhovens, 2018

ISBN: 978-94-6295-858-6

Lay-out by: wenz iD, Wendy Schoneveld

Printed by: ProefschriftMaken || Proefschriftmaken.nl

Financial support by the vestibular society (vestibulaire vereniging). 


\title{
Neurovestibular analysis and falls in Parkinson's disease and atypical parkinsonism
}

Proefschrift ter verkrijging van de graad van doctor aan de Radboud Universiteit Nijmegen

op gezag van de rector magnificus prof. dr. J.H.J.M. van Krieken, volgens besluit van het college van decanen

in het openbaar te verdedigen op

\author{
dinsdag 20 maart 2018 \\ om 10:30 uur precies \\ door \\ Jeroen Venhovens \\ geboren op 14 maart 1981 \\ te Gorinchem
}




\section{Promotor}

Prof. dr. B.R. Bloem

\section{Copromotoren}

Dr. W.I.M. Verhagen (Canisius-Wilhelmina ziekenhuis, Nijmegen)

Dr. J. Meulstee (Canisius-Wilhelmina ziekenhuis, Nijmegen)

\section{Manuscriptcommissie}

Prof. dr. H.A.M. Marres (voorzitter)

Prof. dr. A.C.H. Geurts

Prof. dr. J.E. Bos (Vrije Universiteit Amsterdam) 
Voor mijn vrouw en dochters.

Vandaag is de dag, hij komt maar één keer, morgen dan is het vandaag al niet meer. Niet zeuren, geniet van het leven, het mag, maar doe het vandaag, want vandaag is de dag.

(Toon Hermans, 1916-2000) 


\section{TABLE OF CONTENTS}

Chapter 1 General introduction and outline

\section{PART ONE}

Neurovestibular laboratory testing - practical and technical issues

Chapter 2 Vestibular evoked myogenic potentials (VEMPs) in central neurological disorders

Chapter 3 Ocular and cervical vestibular evoked myogenic potentials (VEMPs) in healthy volunteers: The intra-, interobserver, and the test re-test reliability

Chapter 4 Static Subjective Visual Vertical (SVV) in healthy volunteers: The effects of different preset angle deviations and test re-test variability

\section{PART TWO}

Neurovestibular analysis and falls in Parkinson's disease and atypical parkinsonism

Chapter 5 Neurovestibular analysis and falls in Parkinson's disease and 77 atypical parkinsonism: Results from the baseline study

Chapter 6 Neurovestibular analysis and falls in Parkinson's disease and 101 atypical parkinsonism: Results from the prospective one-year follow-up study 
Chapter 7 Summary, general discussion, and perspectives for future research

Chapter 8 Nederlandse samenvatting, algemene discussie en perspectieven 135 voor toekomstig wetenschappelijk onderzoek

\section{APPENDICES}

List of abbreviations 158

List of publications $\quad 160$

Dankwoord (Acknowledgements) 161

Curriculum Vitae 164 


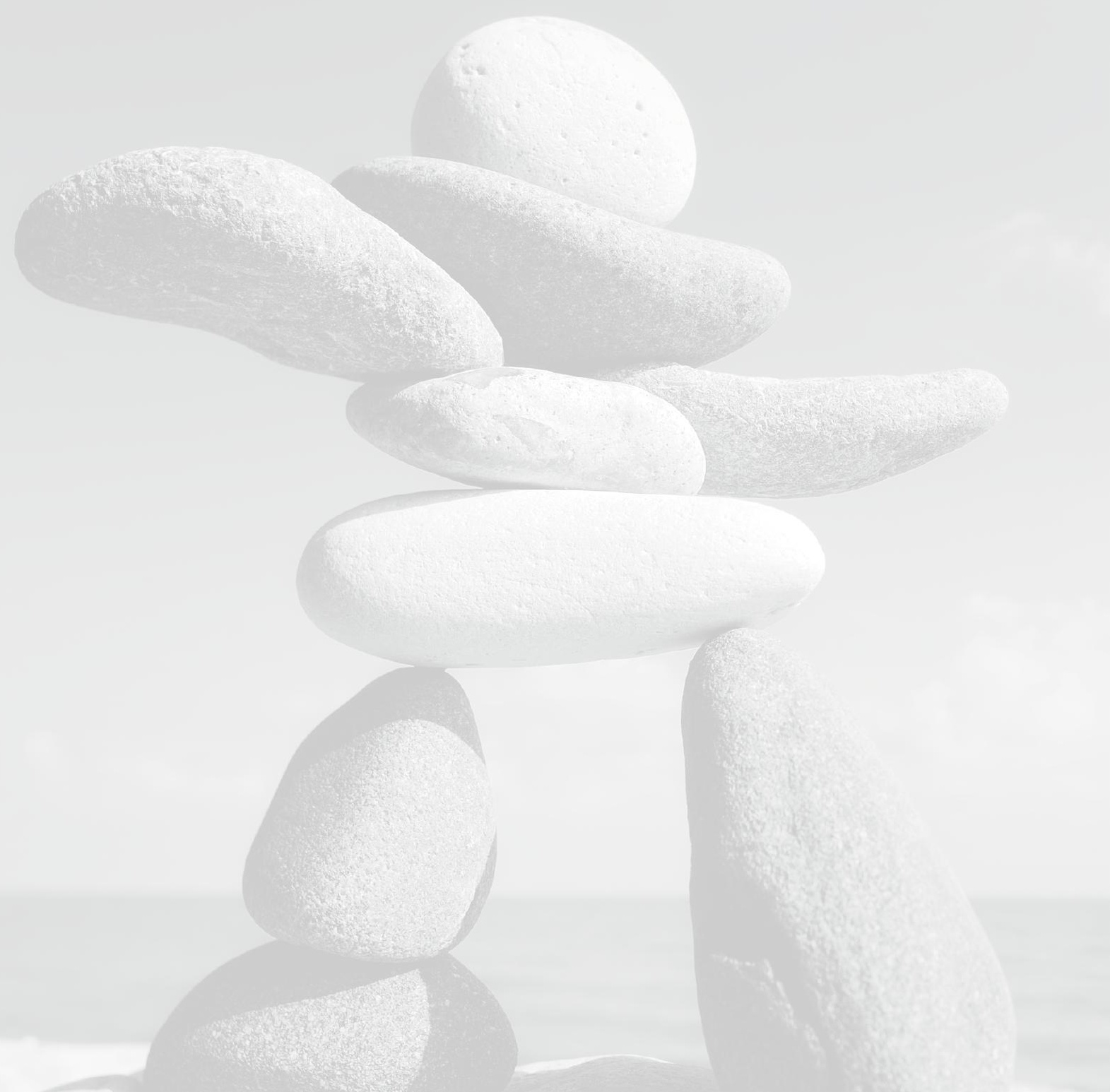




\section{Chapter 1}

General introduction and outline 
The contents of this thesis are divided into two parts. The first part covers both existing and novel neurovestibular laboratory tests, with an emphasis on technical and practical issues. The second part offers a detailed overview of a study concerning neurovestibular analysis in persons with either Parkinson's disease or a form of atypical parkinsonism, performed both at baseline and after one-year prospective follow-up.

\section{PART ONE}

\section{Neurovestibular laboratory testing - practical and technical issues}

The first step in making management decisions in patients with complaints of vertigo and/or dizziness is to find the correct diagnosis. With a few exceptions, it is an illusion that neurovestibular laboratory tests will provide the physician with an aetiological explanation concerning the patient's underlying medical problem. The basis of making the correct diagnosis in neurology in general - and perhaps even more so in patients with neurotological medical problems - is to perform a detailed medical history, and a careful bedside clinical neurological as well as a neurotological examination. These findings will help in creating a clinical context which will then lead to a preliminary diagnosis or a list of differential diagnoses. Subsequently, neurovestibular laboratory tests can be helpful to: a) localize the problem to the peripheral or central vestibular system, b) determine the extent of the lesion, c) assess the status of the compensation process, and d) determine the prognosis in combination with the clinical context ${ }^{1}$. It is often necessary to complement the neurovestibular laboratory tests with additional audiometric analysis, neuroimaging, and/or laboratory examinations in order to make a final diagnosis. In the first part of this thesis, I will discuss a few practical and technical issues of several neurovestibular laboratory examinations (i.e. vestibular evoked myogenic potentials, VEMP; and the subjective visual vertical, SVV), which have not yet been addressed in the literature.

\section{Nystagmography, caloric and rotatory chair stimulation}

Nystagmography is a method for recording eye movements, nystagmus in particular. These recordings can be made in two different ways: a) by using eye goggles with an infrared recording system (i.e. videonystagmography, VNG), or b) by using surface electrodes along the horizontal and vertical axis of the eyes to indirectly determine the eye movements by measuring the changes in the vector of the corneal-retinal dipole (i.e. electronystagmography, ENG) ${ }^{1,2}$. Eye movement recordings are subsequently made: a) during gaze in the neutral midline position and during eccentric 
gaze in all directions, both with and without fixation, b) during saccades at different angles, c) during smooth pursuit with the targets moving at different speeds, d) during optokinetic stimulation with the stimulus moving at different speeds, e) during and/ or after rotatory chair stimulation, and f) after bithermal caloric stimulation with warm and cold water (i.e. 44 and 30 degrees Celsius) or air. Tests a. through d. are used to study the central ocular motor pathways and tests e. and f. are used to dynamically examine the vestibulo-ocular reflex pathways. We refer to the excellent textbook of Baloh and Kerber for a more detailed description of the tests and their interpretation ${ }^{2}$.

\section{Vestibular Evoked Myogenic Potentials (VEMP)}

For the last few decades, VEMP testing has come into use as a test suitable for detailed neurophysiological assessment of both the peripheral and central vestibular system. VEMP testing can be divided into a cervical and ocular response. VEMP responses can be evoked by short intense air conducted sound stimulation, bone conducted vibration, forehead tap stimulation, and galvanic stimulation ${ }^{3-8}$.

Cervical VEMP measurements determine the integrity of the vestibulo-collic reflex pathway starting at the sacculus otolith end-organ through the inferior vestibular nerve, subsequently travelling through the vestibular nuclei and the ipsilateral medial vestibulospinal tract to end in the ipsilateral sternocleidomastoid muscle evoking an inhibitory motor response ${ }^{3,5,6,8,9}$. These responses can be measured by placing an active surface electrode over the upper middle part of the ipsilateral sternocleidomastoid muscle belly and a reference electrode over the sternal manubrium. The sternocleidomastoid muscles, however, have to be tightened before cervical VEMP responses can be elicited by flexing the neck or rotating the head contralaterally to the stimulated ear.

Ocular VEMP measurements determine the integrity of the vestibulo-ocular reflex pathway most likely starting at the utriculus otolith end-organ through the superior vestibular nerve, subsequently travelling through the vestibular nuclei and the medial longitudinal fasciculus to ultimately evoke an excitatory ocular motor response in the contralateral inferior oblique external eye muscle. The utriculus is the most likely otolith end-organ from where the ocular VEMP response originates, yet, this origin is debated extensively in the literature (whether it is the utriculus solely or a combination of both the utriculus and sacculus). However, there is consensus that the relevant afferents travel through the superior vestibular nerve $e^{3,5,6,8,10-13}$. Ocular VEMP responses can be obtained by placing active surface electrodes over the middle 
infra-orbital margin and the reference electrodes 1-2 centimetres below them. The eyes have to be deviated upward during stimulation before ocular VEMP responses can be recorded.

Historically, the clinical neurophysiological VEMP studies focussed on the peripheral vestibular system. Chapter 2 critically reviews the latest VEMP literature concerning central neurological disorders and explains how careful evaluation of the VEMP abnormalities can aid in localizing neurovestibular disorders. I would also like to refer to figure 1, chapter 2, for a schematic representation of the ocular and cervical VEMP pathways. Figure 2, chapter 2, shows an example of the characteristic ocular and cervical VEMP responses in a healthy volunteer, elicited by forehead taps. Chapter 3 offers the results of an intra-, interobserver and test re-test reliability study. Both the intra- and interobserver reliability concerning the assessment of cervical and ocular VEMP responses need to be good or excellent in order for VEMPs to be a reliable clinical diagnostic tool. However, these important technical aspects of clinical VEMP assessment have as yet not been studied before. Also, the test-retest reliability has thus far received rather limited attention in literature, especially concerning ocular VEMPs and forehead tap evoked VEMPs. I would therefore like to refer to the methodology sections of chapters 3 and 5 for a detailed overview concerning the performance of both cervical and ocular VEMP measurements. Fortunately, an international guideline concerning the performance of cervical VEMPs ${ }^{4}$ was published recently.

\section{Subjective Visual Vertical (SVV)}

The static SVV test measures the integrity of the graviceptive pathways. Graviception is the ability of a person to correctly estimate the absolute vertical, being the gravitational force of the earth. These graviceptive pathways integrate the visual, vestibular, and proprioceptive sensory inputs to subsequently estimate the position of the person's body relative to the absolute vertical ${ }^{14-17}$. The SVV test is performed in a completely darkened environment and in an upright sitting position in order to exclude visual references and to minimize proprioceptive inputs. Therefore, the static SVV results are primarily determined by otolithic balance as these are the primary receptors in the body to detect gravitational forces (i.e. linear accelerations) ${ }^{17}$. During SVV testing in our laboratory, subjects are asked to adjust a tilted laser projected line to the absolute vertical by means of a remote controller in a completely darkened environment. Chapter $\mathbf{4}$ has three main technical aims: a) to construct a robust static SVV testing protocol incorporating both binocular and monocular measurements, as SVV testing in literature is severely hampered by the lack of a uniform protocol, making generalization of SVV findings from literature very difficult, b) to study the 
effects of different preset angle deviations and sides, and c) to study the test re-test variability. As noted above the test re-test reliability is an important technical parameter in determining whether the SVV test is a reliable clinical diagnostic tool yet it has thus far received rather limited attention in literature. Also, the size of the preset angle and side of the tilted laser projected line are important technical considerations as these factors can possibly influence the outcome of the SVV test results and have thus far not been studied extensively in literature. However, these technical issues need to be clarified before a uniform static SVV testing protocol could be constructed, which is one of the primary aims of this study.

\section{PART TWO}

\section{Neurovestibular analysis and falls in persons with Parkinson's disease and atypical parkinsonism - a baseline case-control study and prospec- tive follow-up study after one year}

Fall incidents and postural instability are a major problem in persons with Parkinson's disease (PD), and even more so in persons with a form of atypical parkinsonism (AP). Approximately $70 \%$ of PD patients fall once each year, and about $50 \%$ of them experience recurrent fall episodes yearly ${ }^{18-22}$. PD patients have a 2.2-fold increase in fractures overall and a 3 to 4 -fold increase in hip fractures in comparison to an agematched healthy population. Moreover, PD patients with hip fractures have higher rates of surgical and medical post-operative complications (e.g. hospital acquired pneumonia, urinary tract infection, pressure sores), longer post-operative hospital duration, and higher mortality rates ${ }^{23}$. Also, fall incidents in PD patients result in a fear of renewed falls, secondarily leading to social isolation and a reduced quality of life following self-imposed restrictions of daily activities ${ }^{18,20}$.

Recently, a practical consensus-based overview concerning the risk factors for falling in PD patients was published ${ }^{24}$. However, vestibular function abnormalities were not addressed as an individual risk factor, which is surprising since the vestibular system is one of the key systems in maintaining balance by integrating multimodal sensory information (i.e. vestibular, proprioceptive, and visual sensory input) and by secondarily adjusting the outgoing motor response by means of the vestibulospinal reflexes $^{25}$. Moreover, there is very little literature concerning the risk of falling in PD and AP patients in relation to vestibular system abnormalities. Recent work showed that complaints of dizziness are common in patients with PD, and this is related not only to orthostatic hypotension (which most clinicians assume to be the most likely explanation), but also to underlying - and commonly undetected! - benign paroxysmal 
positional vertigo ${ }^{26,27}$. The literature concerning vestibular system abnormalities in $\mathrm{PD}$ and $\mathrm{AP}$ patients in general, too, is very limited and consists of small case-series and case-control studies ${ }^{28-32}$. Chapter 5 presents the results of a case-control study in which the vestibular system function in PD and AP patients was systematically investigated by means of a vestibular testing battery, and the results were compared to an age and gender-matched healthy control group. The results of the vestibular testing battery were subsequently compared to the clinical neurological and neurotological test results and a possible correlation with fall incidents was examined. Chapter $\mathbf{6}$ shows the prospective follow-up results one year after the baseline study as described in chapter five. All patients and healthy volunteers received a telephone interview concerning their falls in the prior year and their fear of falling. The followup results were compared to the baseline results, particularly the results of the baseline vestibular testing battery. The sensitivity and positive predictive values of the individual vestibular function tests were calculated in relation to future fall incidents. 


\section{References}

1. Shepard, N., Janky, K. \& Eggers, S. in Oxford textbook of Vertigo and Imbalance (ed. Bronstein, A. M.) 145-155 (Oxford, University Press, 2013).

2. Baloh, R. W. \& Kerber, K. A. Clinical Neurophysiology of the Vestibular System. (Oxford, University Press, 2011).

3. Curthoys, I. S. A critical review of the neurophysiological evidence underlying clinical vestibular testing using sound, vibration and galvanic stimuli. Clinical Neurophysiology 121, 132-144 (2010).

4. Papathanasiou, E. S., Murofushi, T., Akin, F. W. \& Colebatch, J. G. International guidelines for the clinical application of cervical vestibular evoked myogenic potentials: an expert consensus report. Clin. Neurophysiol. 125, 658-66 (2014).

5. Rosengren, S. M., Welgampola, M. S. \& Colebatch, J. G. Vestibular evoked myogenic potentials: Past, present and future. Clin. Neurophysiol. 121, 636-651 (2010).

6. Rosengren, S. \& Kingma, H. New perspectives on vestibular evoked myogenic potentials. Curr. Opin. Neurol. 26, 74-80 (2013).

7. Welgampola, M. S., Welgampola, M. S., Colebatch, J. G. \& Colebatch, J. G. Characteristics and clinical applications of vestibular-evoked myogenic potentials. Neurology 64, 1682-1688 (2005).

8. Venhovens, J., Meulstee, J. \& Verhagen, W. Vestibular Evoked Myogenic Potentials (VEMPs) in Central Neurological Disorders. Clin. Neurophysiol. 127, 40-49 (2016).

9. Eleftheriadou, A. \& Koudounarakis, E. Vestibular-evoked myogenic potentials eliciting: An overview. European Archives of Oto-Rhino-Laryngology 268, 331-339 (2011).

10. Papathanasiou, E. S. \& Papacostas, S. S. Vestibular evoked myogenic potentials: the fuzzy picture of different stimulation types is beginning to come into focus. Clin. Neurophysiol. 124, 1926-7 (2013).

11. Papathanasiou, E. S. Ocular vestibular evoked myogenic potentials (OVEMPs): saccule or utricle? Clin. Neurophysiol. 123, 216 (2012).

12. Curthoys, I. S. A balanced view of the evidence leads to sound conclusions. A reply to J.G. Colebatch 'Sound conclusions?'. Clin. Neurophysiol. 121, 977-8 (2010).

13. Todd, N. The ocular vestibular evoked myogenic potential (OVEMP), ten years old. Clin. Neurophysiol. 125, 439-41 (2014).

14. Brandt, T. \& Dieterich, M. Vestibular syndromes in the roll plane: topographic diagnosis from brainstem to cortex. Ann. Neurol. 36, 337-47 (1994).

15. Tarnutzer, a a, Bockisch, C., Straumann, D. \& Olasagasti, I. Gravity dependence of subjective visual vertical variability. J. Neurophysiol. 102, 1657-1671 (2009).

16. Yelnik, A. P. et al. Perception of verticality after recent cerebral hemispheric stroke. Stroke 33, 2247-2253 (2002).

17. Venhovens, J., Meulstee, J. \& Verhagen, W. I. M. Static Subjective Visual Vertical in Healthy Volunteers: The Effects of Different Preset Angle Deviations and Test-Retest Variability. Neuroophthalmology. 40, 113-119 (2016).

18. Grimbergen, Y. A. M., Munneke, M. \& Bloem, B. R. Falls in Parkinson's disease. Curr. Opin. Neurol. 17, 405-415 (2004).

19. Bloem, B. R., Grimbergen, Y. A., Cramer, M., Willemsen, M. \& Zwinderman, A. H. Prospective assessment of falls in Parkinson's disease. J. Neurol. 248, 950-8 (2001).

20. Bloem, B. \& Bhatia, K. in Clinical disorders of balance, posture and gait. (ed. Bronstein AM, Brandt T, Nutt JG, W. M.) 173-206 (Arnold: London, 2004).

21. Wood, B. H., Bilclough, J. A., Bowron, A. \& Walker, R. W. Incidence and prediction of falls in Parkinson's disease: a prospective multidisciplinary study. J. Neurol. Neurosurg. Psychiatry 72, 721 725 (2002).

22. Bloem, B. R., Steijns, J. A. G. \& Smits-Engelsman, B. C. An update on falls. Curr. Opin. Neurol. 16, 15-26 (2003).

23. Critchley, R. J., Khan, S. K., Yarnall, A. J., Parker, M. J. \& Deehan, D. J. Occurrence, management and outcomes of hip fractures in patients with Parkinson's disease. Br. Med. Bull. 115, 135-42 (2015). 
24. van der Marck, M. A. et al. Consensus-based clinical practice recommendations for the examination and management of falls in patients with Parkinson's disease. Parkinsonism Relat. Disord. 20, 360 9 (2014).

25. Venhovens, J., Meulstee, J., Bloem, B. R. \& Verhagen, W. I. M. Neurovestibular analysis and falls in Parkinson's disease and atypical parkinsonism. Eur. J. Neurosci. 43, 1636-46 (2016).

26. van Wensen, E., van Leeuwen, R. B., van der Zaag-Loonen, H. J., Masius-Olthof, S. \& Bloem, B. R. Benign paroxysmal positional vertigo in Parkinson's disease. Parkinsonism Relat. Disord. 19, 1110-2 (2013).

27. Becker-Bense, S. et al. Prevalence of Parkinson symptoms in patients with different peripheral vestibular disorders. J. Neurol. 264, 1287-1289 (2017).

28. Wang, S. R. \& Young, Y. H. Multiple system atrophy manifested as dizziness and imbalance: A report of two cases. Eur. Arch. Oto-Rhino-Laryngology 260, 404-407 (2003).

29. Bassetto, J. M., Zeigelboim, B. S., Jurkiewicz, A. L. \& Klagenberg, K. F. Neurotological findings in patients with Parkinson's disease. Braz J Otorhinolaryngol 74, 350-355 (2008).

30. Liao, K. et al. Why do patients with PSP fall? Evidence for abnormal otolith responses. Neurology 70, 802-809 (2008).

31. Pollak, L., Prohorov, T., Kushnir, M. \& Rabey, M. Vestibulocervical reflexes in idiopathic Parkinson disease. Neurophysiol. Clin. 39, 235-240 (2009).

32. Pötter-Nerger, M., Govender, S., Deuschl, G., Volkmann, J. \& Colebatch, J. G. Selective changes of ocular vestibular myogenic potentials in Parkinson's disease. Mov. Disord. 30, 584-9 (2015). 


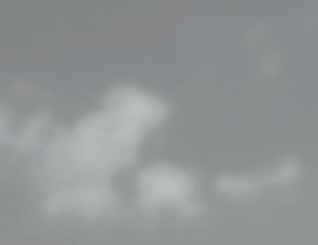

$-\infty$

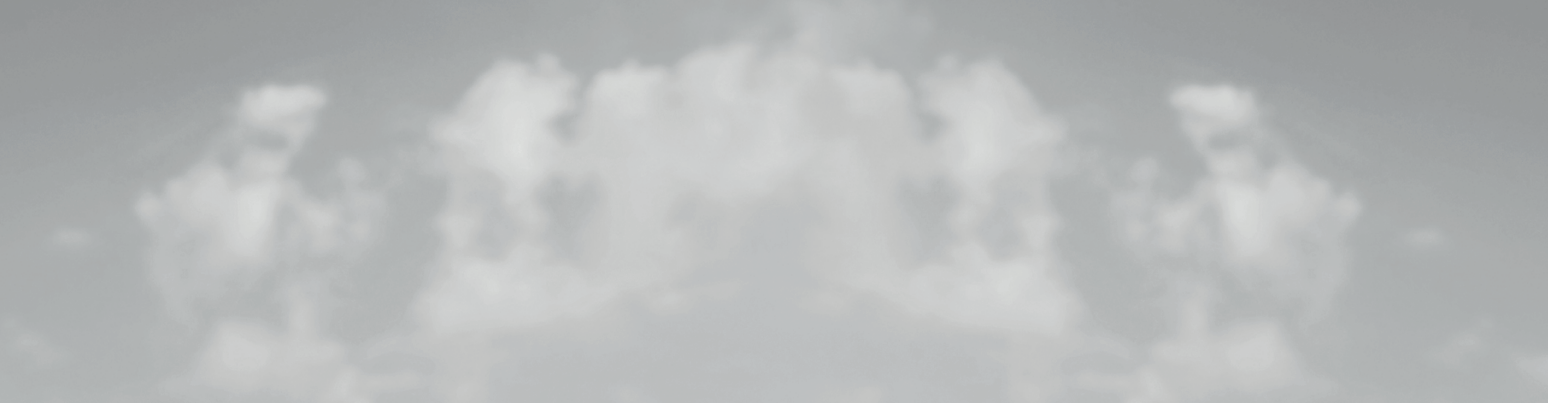




\section{PART ONE}

Neurovestibular laboratory testing Practical and technical issues

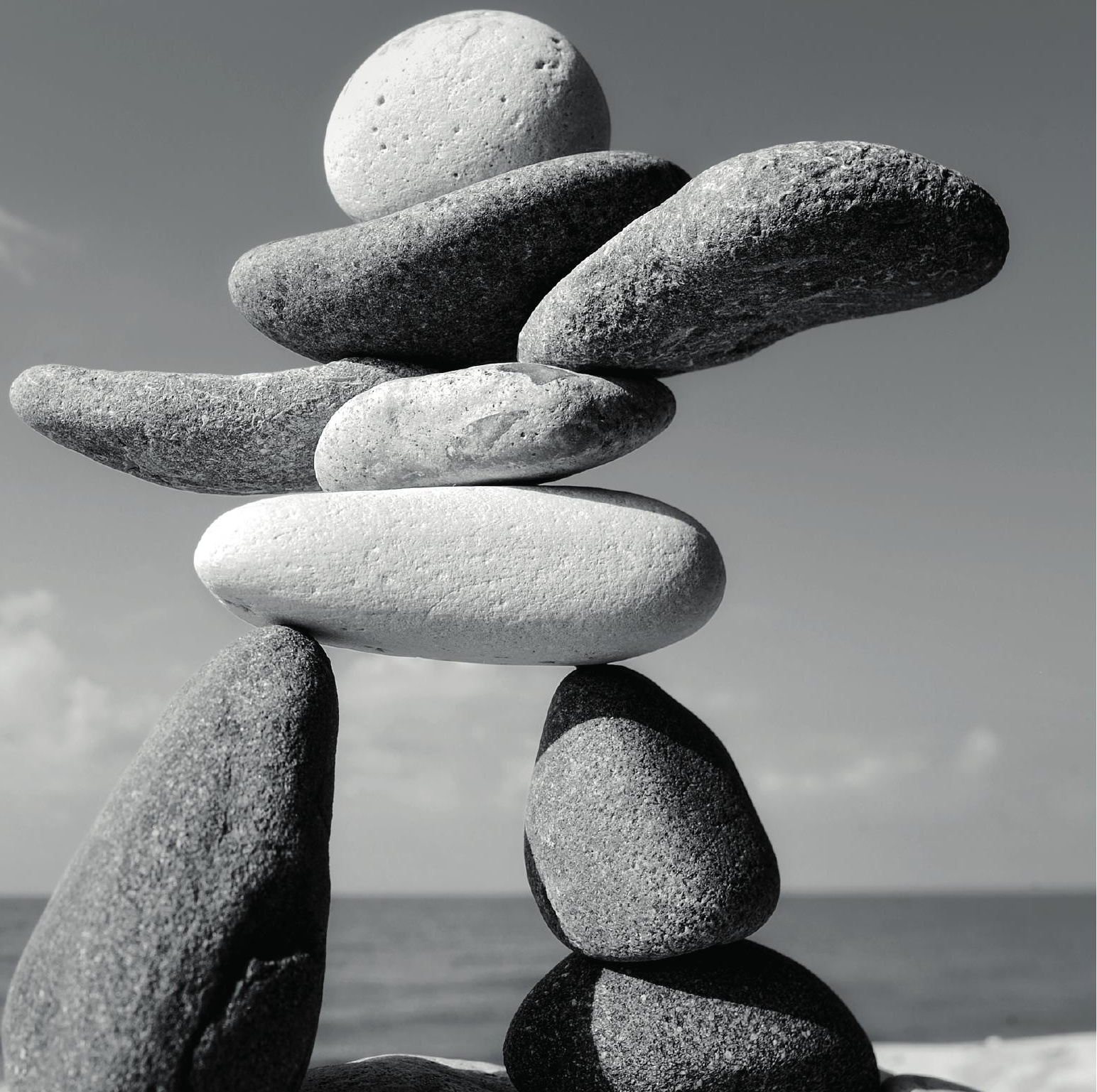




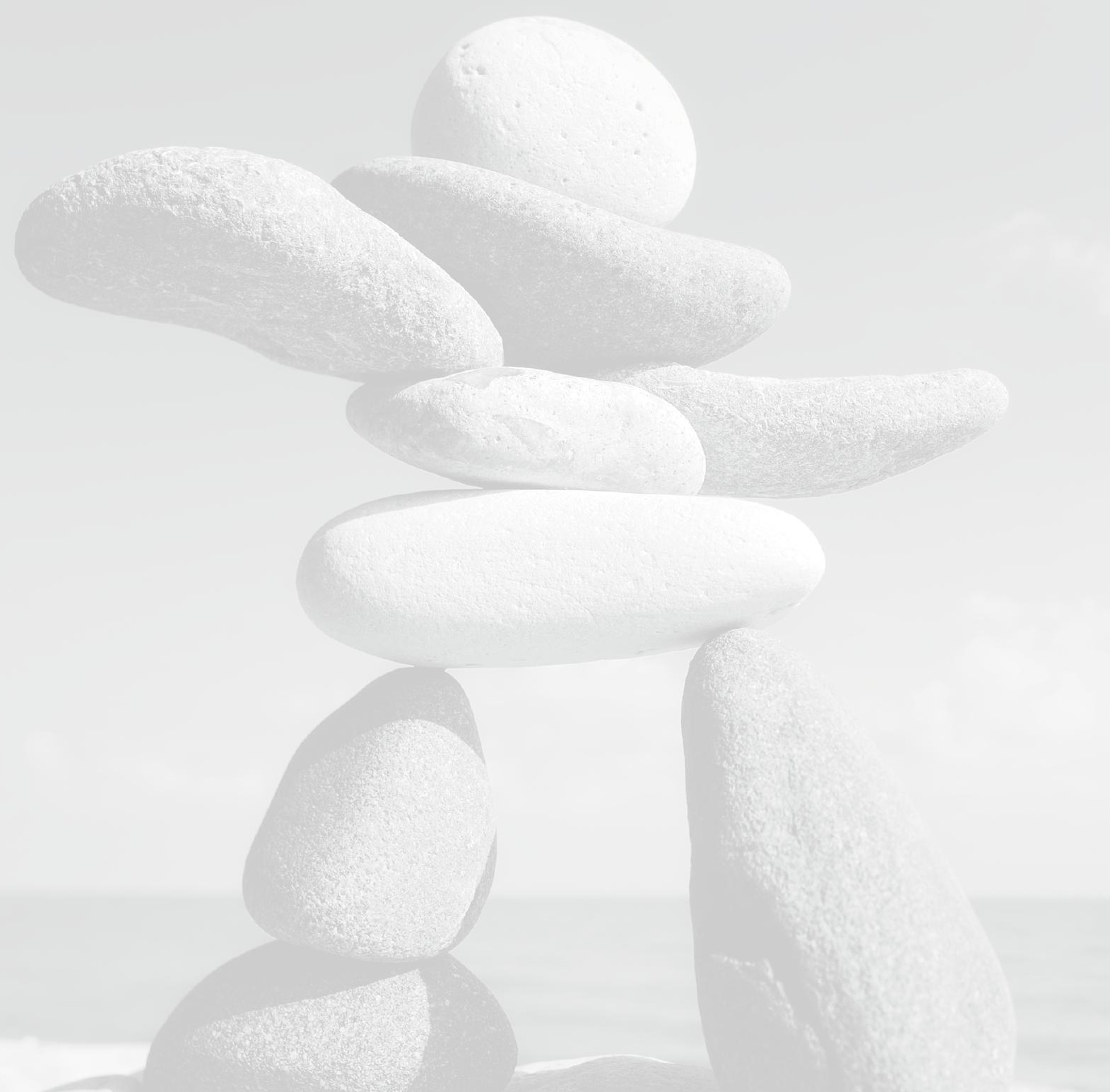




\section{Chapter 2}

Vestibular evoked myogenic potentials (VEMPs) in central neurological disorders

J. Venhovens, J. Meulstee, W.I.M. Verhagen

Clin. Neurophysiol. 127, 40-49 (2016) 


\section{Abstract (review)}

Several types of acoustic stimulation (i.e. tone bursts or clicks), bone-conducted vibration, forehead taps, and galvanic stimulation may give rise to myogenic potentials. These may be recorded in cervical and ocular muscles, the so called vestibular evoked myogenic potentials (VEMPs). The cervical VEMP (cVEMP) resembles the vestibulo-collic reflex and the responses may be recorded from the ipsilateral sternocleidomastoid muscle. The ocular VEMP (oVEMP) resembles the vestibulo-ocular reflex and may be recorded from extra-ocular muscles by a surface electrode beneath the contralateral infraorbital margin. Initially, the literature concerning VEMPs was limited to peripheral vestibular disorders, however, the field of VEMP testing is rapidly expanding with an increasing interest for central neurological disorders. The current literature concerning VEMP abnormalities in central neurological disorders is critically reviewed, especially regarding the methodological aspects in relation to quality as well as clinical interpretation of the VEMP results. Suggestions for further research are proposed as well as some clinical useful indications. 


\section{Introduction}

In the last two decades vestibular evoked myogenic potentials (VEMPs) emerged as a test suitable for detailed physiological assessment of the vestibular system. VEMPs can be evoked by short intense auditory stimuli (i.e. tone bursts or clicks), bone-conducted vibration, forehead taps, and galvanic stimulation ${ }^{1-5}$. In daily practice, air-conducted acoustic stimuli are used most often, followed by bone-conducted vibration and forehead taps. The responses can be divided into a cervical response and an ocular response.

The cervical vestibular evoked myogenic potential (cVEMPs) can be recorded by placing an active surface electrode over the upper middle part of the sternocleidomastoid muscles with a reference electrode on the sternal manubrium. The sternocleidomastoid muscles, however, both have to be activated by flexing the neck, or have to be ipslaterally activated by rotating the head contralaterally away from the stimulated ear (i.e. when cVEMPs are acoustically elicited by air-conducted clicks or tone bursts, AC-cVEMPs). cVEMPs check the integrity of the vestibulo-collic reflex from the saccular afferents to the brainstem vestibular nuclei (through the inferior vestibular nerve), evoking an inhibitory motor response in the ipsilateral sternocleidomastoid muscle (through the medial vestibulospinal tract, upper cervical motor neurons and the accessory nerve) $)^{1,3,4,6}$ (figure 1 ).

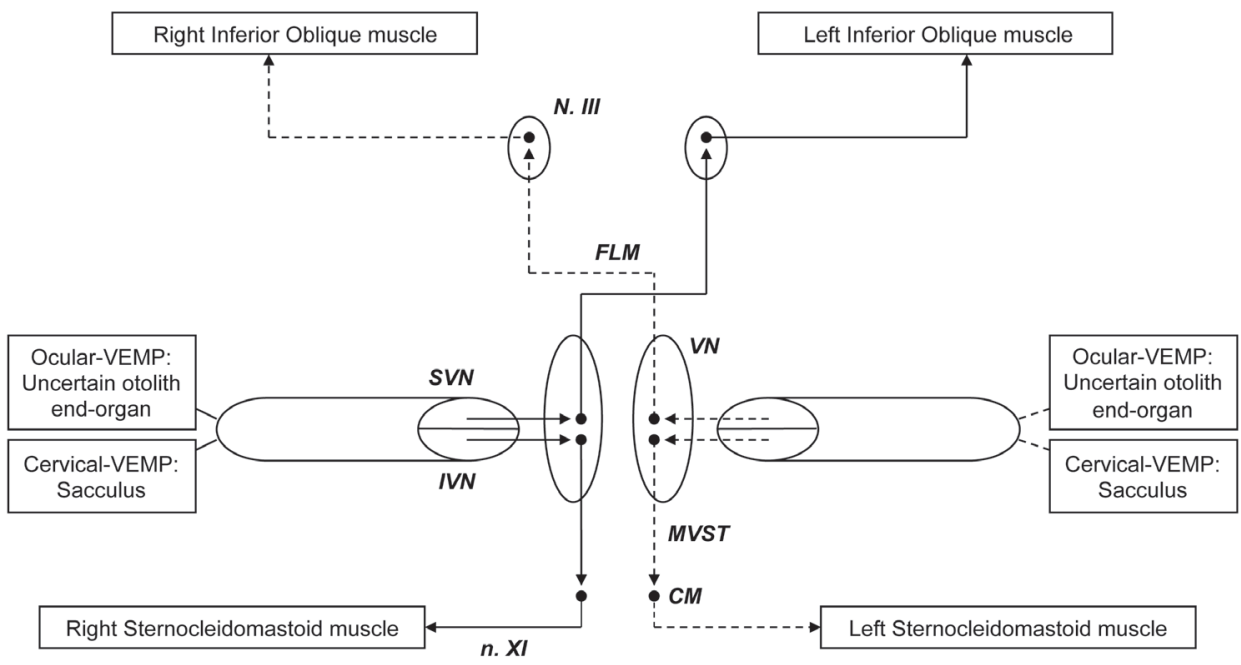

Figure 1.

Neurophysiological pathways concerning the ocular and cervical vestibular evoked myogenic potentials. We refer to the text for further explanation. CM: cervical motor neuron; FLM: medial longitudal fasciculus; $I V N$ : inferior division of the vestibular nerve; MVST: medial vestibulospinal tract; $N$. III: oculomotor nucleus; $n$. XI: accessory nerve; $S V N$ : superior division of the vestibular nerve; $V N$ : vestibular nuclei. 
The ocular vestibular evoked myogenic potential (oVEMP) measures the function of the vestibulo-ocular reflex arc from the otolith end-organ(s) to the brainstem vestibular nuclei through the superior vestibular nerve and medial longitudal fasciculus, evoking an excitatory oculomotor response in the inferior oblique muscle mainly in the contralateral eye. The precise localisation of the end-organ involved in the origin of the oVEMP is controversial and extensively debated in the literature (i.e. whether the end-organ is predominantly the sacculus, the utriculus, or a combination of both), however there is consensus that the relevant afferents travel through the superior division of the vestibular nerve ${ }^{1,3,4,6-11}$ (figure 1). By maintaining an upward gaze during the stimulation oVEMPs can be recorded by an active electrode just below the middle of the infraorbital margin and the reference electrodes 1-2 centimetres below the active electrode $e^{1,12}$.

The cVEMPs morphology can be divided into two parts. The early biphasic positivenegative component (p13-n23) is presumed to be of mainly saccular origin. The second biphasic negative-positive complex (n34-p44) is thought to be auditory in acoustic stimulation and of an unknown origin in forehead tap evoked responses (possibly a stretch reflex) (figure 2). The oVEMPs morphology consists of a quadriphasic negative-positive deflection (n1-p1 and n2-p2 complex) (figure 2).
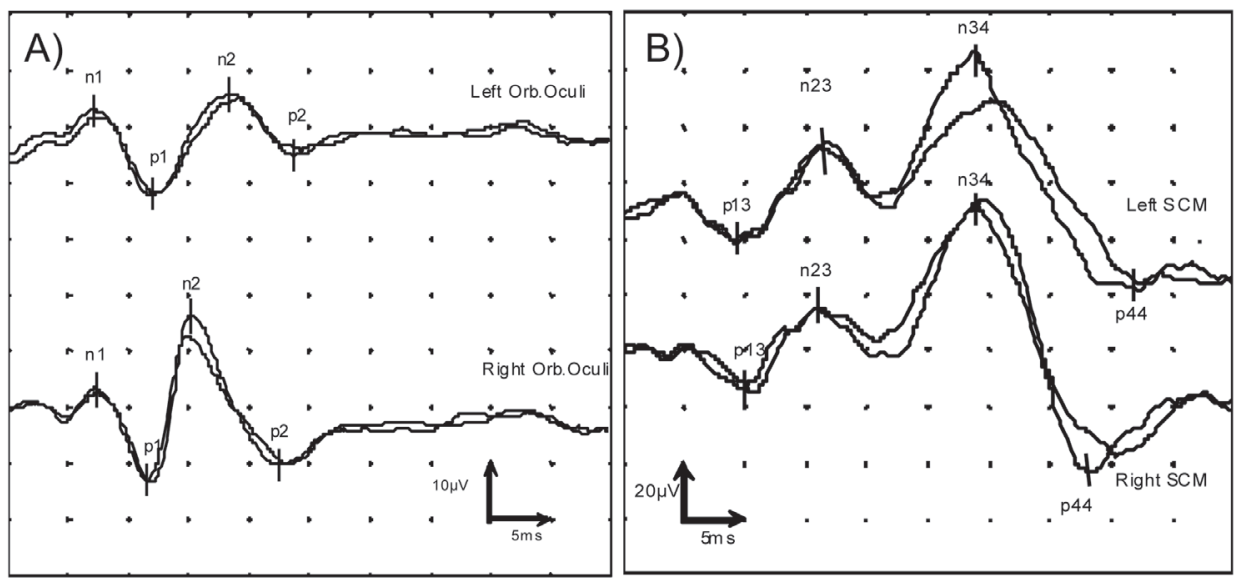

Figure 2.

A: Ocular vestibular evoked myogenic potentials in a healthy subject elicited by forehead taps with the typical quadriphasic negative-positive-negative-positive response (n1-p1-n2-p2) with normal latencies and amplitudes. B: Cervical vestibular evoked myogenic potentials in a healthy subject elicited by forehead taps with a typical biphasic p13-n23 response of vestibular origin followed by a biphasic n34-p44 biphasic response with normal latencies and amplitudes. (Both tap-evoked oVEMP and cVEMP responses were obtained in our own neurovestibular research laboratory) ${ }^{*}$ The trigger delay, as used in forehead tap evoked ocular and cervical vestibular evoked myogenic potentials, apparently leads to the shortening of both oVEMP and cVEMP latencies (e.g. when compared to acoustically elicited oVEMP and cVEMP latencies). 
Historically the clinical research concerning VEMPs has so far focused on peripheral neurovestibular disorders. However the field of VEMP testing is rapidly expanding with an increasing interest for central neurological disorders. For an overview on VEMP abnormalities in mainly peripheral vestibular disorders we refer to Brantberg $(2009)^{13}$. Reviews concerning VEMP abnormalities in central neurologic conditions are scarce, despite the growing number of clinical studies. In this paper, we present an overview concerning VEMP abnormalities in central neurological disorders, the interpretation of these VEMP results, and clinical application. In addition we stress the importance of applying a correct methodological procedure in future clinical VEMP research and clinical VEMP testing in individual patients. Therefore we critically assessed the available literature concerning VEMP abnormalities in central neurological disorders to get an impression of the applied technical aspects as well as the methodology and the interpretation of the studies in order to estimate the level of diagnostic evidence.

\section{Demyelinating Disease}

Multiple sclerosis (MS) is a chronic, demyelinating disease of the central nervous system. MS is a leading cause of disability in young adults with a prevalence of 1 out of 1000 persons living in northern Europe ${ }^{14}$.

The presence of AC-cVEMP abnormalities varies from 31 to 70 percent in the literature; delayed p13 often in combination with increased $\mathrm{n} 23$ latencies are the most common abnormalities, followed by absent responses. There was a significant correlation between abnormal AC-cVEMPs and vestibular symptoms, expanded disability status scale (EDSS) equal or greater than 5.5, EDSS brainstem functional system score equal or greater than 1 , and a longer disease duration (i.e. disease duration more than 10 years) $)^{15-24}$.

Recently, studies of acoustic air-conducted ocular vestibular evoked myogenic potentials were reported (AC-oVEMPs). The presence of abnormal responses ranged from 37.5 to 69 percent ${ }^{19,21,25}$. Increased latency of the response was the most prevalent abnormality followed by absent AC-oVEMPs. Rosengren and Colebatch (2011)25 studied AC-oVEMPs in thirteen patients (twelve of whom had MS and one had a presumed vascular aetiology) with internuclear ophthalmoplegia (INO). In the patients with a unilateral INO three out of five (60 percent) had abnormal ACoVEMPs. In one patient the side of the INO was in concordance with the absent AC-oVEMPs response and in the other two patients AC-oVEMPs were bilaterally absent or delayed. All of the eight tested patients with bilateral INO showed abnormal AC-oVEMPs (five patients showed bilaterally abnormal AC-oVEMPs responses and three patients showed unilaterally abnormal AC-oVEMPs responses). The patients 
with a partial INO (slowing of ipsilesional ocular adduction rather than failure of adduction) had prolonged latencies of the AC-oVEMPs, and patients with a complete INO showed absent AC-oVEMPs ${ }^{25}$.

Most of these studies include a limited number of patients (i.e. mostly between the 20 and 40 patients with a few exceptions). The variation in sensitivity between the studies could possibly be explained by a difference in patient's disease characteristics (i.e. some studies contain substantially more multiple sclerosis patients with a longer disease duration, or have more patients with progressive forms of multiple sclerosis compared to other studies). Most studies ${ }^{15,16,18,20-23}$ also did not correct the cVEMP amplitudes (i.e. by using the rectified mean pre-stimulus EMG background signal at the desired level of sternocleidomastoid muscle contraction to correct the raw p13-n23 amplitude from the unrectified EMG signal), or by standardizing the level of sternocleidomastoid muscle contraction (e.g. for instance with a pressure gauge to assure that the same level of force is applied during muscle contraction at both sides and between measurements as described by Vanspauwen et al. 2006) ${ }^{26,27}$. The raw p13-n23 cVEMP amplitudes cannot be reliably compared between groups, sides within the same patient, or between measurements within the same patient at different moments if standardization and correcting methods, as described above, are not used (i.e. changes in the level of muscle contraction will influence the raw p13-n23 amplitude) $)^{3,28}$. Sartucci and Logi (2002) ${ }^{23}$ examined cVEMPs in fifteen MS patients; the control group consisted of fifteen volunteers in whom the mean age was considerably lower than the MS group (i.e. $44.5 \pm 10.3$ years in the MS group, and $26.7 \pm 3.4$ years in the control group). The difference in the raw $\mathrm{p}_{13} \mathrm{n}_{23}$ amplitudes between groups was significantly different (i.e. with lower amplitudes in the MS group). However, the cVEMP amplitudes, especially when acoustically elicited, decrease with advancing age ${ }^{29,30}$, so differences in p13-n23 amplitudes should be interpreted with caution when the study group and control group are not agematched.

The hypothesis is that relatively small demyelinating lesions in the brainstem can cause conduction slowing in the vestibulo-spinal and vestibulo-ocular pathways resulting in prolonged latencies with a more or less preserved configuration of the responses. When demyelination is more pronounced, a partial conduction block may occur (possibly in combination with axonal damage) and a lack of synchronisation will induce phase cancellation and a decrease in amplitude and prolongation of latencies of the vestibulo-ocular and vestibulo-spinal responses. More severe demyelination eventually accompanied by secondary axonal loss will eventually result in a total conduction block with absence of responses ${ }^{18,22,23,25}$. 
In our opinion cVEMP and oVEMP may be useful in the evaluation of brainstem involvement in MS patients with neurovestibular complaints without brainstem lesions on cerebral MRI. So in our clinic VEMPs are used in these patients as a complementary diagnostic tool when the cerebral MRI is not explanatory. At this moment there is no place for standard VEMP testing in all MS patients, or in the work-up of new suspected MS patients due to the lack of treatment consequences. VEMP research can be relevant in evaluating brainstem involvement in early MS and clinically isolated syndromes or during the course of the disease for further pathophysiological clarification and may play a part in selecting those patients with clinically isolated syndromes that would benefit from early immunomodulatory treatment.

\section{Cerebrovascular Disease}

Ischemic stroke occurring in the vascular territory of the vertebrobasilar arterial system accounts for approximately 20 percent of all ischemic strokes. The most common aetiologies are atherosclerotic large and/or small vessel disease. Vertebrobasilar stroke can present with a variety of symptoms such as vertigo, ataxia, dysphagia, hearing loss, diplopia, alterations of consciousness, and hemiparesis which may rapidly progress to quadriparesis or locked-in-syndrome ${ }^{31}$. Several small case series in the literature describe VEMP abnormalities in patients with recent vertebrobasilar artery strokes. Abnormalities of AC-cVEMPs in localized brainstem strokes range from 11 percent to 100 percent, however most reside between the 40 and 50 percent; for BCV-oVEMPs and AC-oVEMPs the range is from 50 to 80 percent. Most VEMP abnormalities consist of absent responses followed by a diminished amplitude, however, delayed latencies were described in a few patients ${ }^{32-40}$. Kim et al. $(2014)^{35}$ described AC-oVEMP and AC-cVEMP studies in twelve patients with isolated internuclear ophthalmoplegias due to vascular origin. Eight of the twelve patients (67 percent) had abnormal ACoVEMPs and 25 percent had abnormal AC-cVEMPs. The abnormal AC-oVEMPs were on the lesion side in 86 percent of the patients (seven out of eight patients) in combination with a contraversive ocular tilt reaction (i.e. triad consisting of skewdeviation, ocular torsion and head tilt ${ }^{35}$. In another case series by Oh et al. $(2013)^{36}$ fifty-two patients with acute brainstem strokes were studied with AC-oVEMPs. Out of five patients with midbrain strokes, four had abnormal AC-oVEMPs, mostly in combination with a contraversive ocular tilt reaction and tilt in the subjective visual vertical. Out of twenty-eight patients with a pontine stroke, sixteen (57 percent) had abnormal AC-oVEMPs. Responses in medullary strokes were abnormal in 47 percent of the patients. The authors concluded that abnormal AC-oVEMPs are associated with lesions of the dorsomedial tegmentum from the upper medulla oblongata to the 
midbrain, where the medial longitudinal fasciculus, the crossed ventral tegmental tract, oculomotor nuclei, and oculomotor neurons are located ${ }^{36}$.

VEMP studies in cerebellar infarctions concern small case series and most patients also have concomitant brainstem involvement ${ }^{32,34,37,38,40}$. Weng and Young $(2014)^{40}$ studied AC-cVEMPs and BCV-oVEMPs in eighteen patients with anterior inferior cerebellar artery (AICA) strokes and posterior inferior cerebellar artery (PICA) strokes. AC-cVEMPs were abnormal in 50 percent in PICA stroke patients and 66 percent in AICA stroke patients; while BCV-oVEMPs were abnormal in 86 percent and 100 percent, respectively (i.e. only one AICA patient had BCV-oVEMPs testing which was abnormal). Abnormal caloric testing in combination with abnormal visual suppression tests were present in 93 percent of the patients. Electronystagmography also showed high rates of abnormal visual pursuit, saccade, and optokinetic nystagmus disturbances in both stroke groups varying from 67 to 100 percent. There was, however, a significant difference between the two groups concerning hearing loss complaints and documented mean hearing levels greater than 25 decibels (i.e. 100 percent of the AICA stroke patients and 9 percent of the PICA stroke patients, respectively $)^{40}$. From this it can be concluded that the higher AC-cVEMPs and BCVoVEMPs abnormality rate in AICA stroke patients may partially be explained by labyrinthine ischemia (because the proximal branches of the AICA supply the labyrinthine artery). This conclusion is supported by the result of Ahn et al. (2011) 32 who studied AC-cVEMPs in sixteen AICA stroke patients. Eight out of sixteen patients (50 percent) had abnormal AC-cVEMPs (i.e. absent responses or a decrease in amplitude). In the AICA stroke group with abnormal AC-cVEMPs 75 percent had sensorineural hearing loss and a canal paresis on caloric testing versus none of the patients with normal AC-oVEMPs (however, 25 percent of the latter group had an isolated sensorineural hearing loss and 13 percent had an isolated canal paresis $)^{32}$.

Choi et al. (2014) ${ }^{34}$ studied AC-cVEMPs and BCV-oVEMPs in patients with acute unilateral cerebellar infarction (i.e. PICA strokes in 63 percent, combined PICA and superior cerebellar artery strokes in 26 percent, superior cerebellar artery strokes in 7 percent, and AICA strokes in 4 percent). The patients with an ocular tilt reaction had significantly more AC-cVEMP and BCV-oVEMP abnormalities versus patients without an ocular tilt reaction (eleven out of fifteen patients versus three out of twelve patients, respectively) ${ }^{34}$. The authors concluded that the cerebellum has lateralized effects on the otolithic modulation as shown by the asymmetric VEMP responses in unilateral cerebellar lesions. However, the absence of correlation between the directionality in the ocular tilt reaction and the VEMP abnormalities concerning the lesion side suggests deactivation or disinhibition of the cerebellar structures involved in otolithic modulation ${ }^{34}$. However in a small case series by Su and Young (2011) 38 
oVEMPs and cVEMPs in all patients with localized cerebellar lesions (i.e. three patients with a localized cerebellar meningioma, and one patient with a metastatic adenocarcinoma, all without brainstem involvement on MRI) were normal. This in contrast to the other eight patients with cerebellar lesions and adjacent brainstem involvement, whom showed a high degree of VEMP abnormalities (i.e. 88 percent of the oVEMPs where abnormal, and 75 percent of the cVEMPs were abnormal). Also Pollak et al. (2006) ${ }^{37}$ could not demonstrate significant cVEMP differences in patients with a cerebellar stroke. The topic whether localized cerebellar lesions without adjacent brainstem involvement cause VEMP abnormalities has therefore yet to be clarified, with additional research needed.

Miwa et al. (2013) ${ }^{41}$ described five patients with superficial siderosis of the central nervous system (SSCNS). This disorder is the result of iron deposition from hemosiderin onto the surface of the brain and around some of the cranial nerves (i.e. the nerves and brain tissue areas rich in microglia as they synthesize ferritin) due to continued bleeding into the subarachnoid space. SSCNS is clinically characterized by sensorineural hearing loss, ataxia, dizziness, pyramidal signs and dementia. ACcVEMPs were abnormal in three out of five patients and calorics were abnormal in all patients. There was a correlation between the results of AC-cVEMP measurements and the caloric testing; when AC-cVEMPs were absent, the caloric testing showed areflexia. When the AC-cVEMPs were normal, caloric testing showed hyporeflexia. There also was a correlation between the duration of the disease and vestibular function testing. Disease duration ranging from weeks to months showed normal AC-cVEMPs and a duration longer than several years showed absent AC-cVEMPs. This could be the result of secondary fibre tract degeneration. Brain MRI, however, showed hemosiderin depositions around the vestibulocochlear nerve in just one patient, so the authors concluded that the symptoms and vestibular dysfunction in SSCNS are probably the result of a peripheral labyrinthine problem due to secondary decreased blood flow ${ }^{41}$. Ushio et al. (2006) ${ }^{42}$ described a patient with SSCNS for a period of 21 years who also had absent AC-cVEMPs.

Most studies however share the same methodological problems as described earlier in the chapter on demyelinating disorders, namely that a) cVEMP raw p13-n23 amplitudes were neither corrected nor was the level of muscle contraction standardized ${ }^{32,34,37-41,43}$, and b) most small case series do not have a control group, whereby the laboratory's own normative data were used as a reference ${ }^{36,39-41,43}$, from which the group characteristics (e.g. (mean) age) were unknown. Therefore amplitude differences between the groups or inter-aural differences should be interpreted with caution. Also Choi et al. (2014) ${ }^{34}$ described remarkable cVEMP and oVEMP amplitude differences (i.e. oVEMP amplitudes were about five times higher than the 
cVEMP amplitudes in both the control group and the patient group), whereas mostly the cVEMP amplitudes in the literature and in our laboratory are much higher than the oVEMP amplitudes.

In our opinion VEMP testing should not be performed in the standard work-up of all stroke patients. However when confronted with a patient with acute vertigo, it can be difficult sometimes to differentiate between a peripheral or central neurovestibular disorder. Kattah et al. (2009) ${ }^{44}$ described the HINTS examination (i.e. normal headimpulse test, direction-changing nystagmus, and/or skew deviation) which, as stated by the authors, has a high sensitivity of 100 percent and high specificity of 96 percent for the clinical detection of a vestibular disorder of central neurological origin in patients with a moderate to high risk acute vestibular syndrome (AVS). However, the study has some methodological issues whereby some patients with an AVS could possibly have been falsely classified as having a peripheral vestibular disorder instead of a central one. The peripheral AVS group in the study was defined as, a) the absence of acute stroke in the brainstem or cerebellum by MRI with diffusion-weighted imaging (DWI), b) lack of clinical signs on serial examination, and c) characteristic clinical course. However, this study and other studies ${ }^{44-46}$ have concluded that cerebral MRI with DWI has a limited sensitivity of about 90 percent for the detection of brainstem infarctions. Also, a) patients in the peripheral AVS group were not followed with serial MRI scans in contrast to some patients of the central AVS group, and b) the authors stated that the diagnosis of the peripheral AVS group was confirmed by caloric testing. However, caloric testing showed vestibular paresis in nineteen of the twenty-five patients (i.e. which is highly suggestive of a peripheral vestibular disorder), but showed a directional preponderance in three patients (i.e. which gives no information to the localisation of the problem concerning a possible peripheral or central origin), and could not be performed in another three patients. Therefore the diagnosis of a peripheral origin AVS could not be confirmed in six of the twenty-five patients (i.e. 24 percent), which could, in combination with the lack of serial MRI follow-up, have potentially lead to the misclassification of AVS patients (i.e. to the peripheral AVS group instead of the central origin group) and a falsely high sensitivity of the HINTS examination. Also, the HINTS examination was performed by an experienced neuro-ophthalmological subspecialist, and only moderate to high risk AVS patients were included in the study. Therefore the findings of this study cannot be easily generalized to the everyday practice of patients with acute vertigo visiting the emergency department. Additional VEMP testing possibly in combination with videonystagmography testing and calorics can be helpful in localizing the lesion in individual patients (with normal cerebral MRI with DWI results). VEMPs also are important for research. The issue of VEMP abnormalities in localized cerebellar lesions still has to be clarified as the literature on this topic has shown conflicting results. 
VEMPs might also be applied to assess the risk of falling in the individual patient. However the additional prognostic value of VEMP testing to assess the risk of falling has to be studied prospectively in a large cohort.

\section{Neurodegenerative Disease}

The literature concerning VEMPs in neurodegenerative disorders is very scarce. Takegoshi and Murofushi (2000) ${ }^{47}$ studied collic VEMPs in spinocerebellar degeneration. Ten patients with olivo-ponto-cerebellar atrophy (OPCA), three patients with cortical cerebellar atrophy (CCA), and three patients with Machado-Joseph disease (MJD) were studied with AC-cVEMPs and caloric testing. Machado-Joseph disease is also known as spinocerebellar ataxia type 3. MJD is an autosomal dominantly inherited disease (i.e. expansion of abnormal CAG trinucleotide repeats in the Ataxin-3 gene) which is characterized by cerebellar ataxia, pyramidal signs and progressive external ophthalmoplegia with square wave jerks. The disease is, to varying degrees, also associated with symptoms of polyneuropathy (sensory more than motor), bulging eyes, dystonia, and diplopia. OPCA is characterized by progressive cerebellar ataxia in combination with pyramidal and extrapyramidal signs whereas CCA only has signs of a pure progressive cerebellar ataxia without any additional symptoms. Patients with OPCA and CCA had reasonably well preserved AC-cVEMPs but in MJD VEMPs were abnormal in two out of three patients; in one patient AC-cVEMPs were bilaterally absent, in another AC-cVEMPs were absent on one side and significantly prolonged on the other side. The AC-VEMPs correlated with the caloric responses in these patients; when the AC-cVEMPs were absent, the caloric responses were absent too, and the caloric response in the patient with delayed AC-cVEMPs was decreased $)^{47}$.

The study performed by Takegoshi and Murofushi $(2000)^{47}$ has three methodological shortcomings which are, a) the study population is very small, however it should be noted that the studied spinocerebellar disorders are rare therefore making it difficult to obtain larger groups, b) the subjects in the patient group were not matched for age with the control group, and c) the level of muscle contraction of the sternocleidomastoid muscles was neither standardized nor was the raw p13-n23 amplitude corrected, as discussed above.

Pollak et al. (2009) ${ }^{48}$ described AC-cVEMP studies in fifty-four Parkinson's disease patients. In this group twenty patients had unilaterally absent responses (37 percent) and four had bilaterally absent responses (7.4 percent). However, mean peak latencies did not significantly differ between the groups with preserved AC-cVEMPs. There 
was no significant correlation between AC-cVEMPs and demographic features, disease characteristics and treatment modalities. Patients on anti-depressive agents, however, had a higher chance of having abnormal AC-cVEMPs; fifteen of the twenty-four patients with depression and anti-depressive medication had abnormal AC-cVEMPs, which was significant.

Progressive supranuclear palsy (PSP) is a parkinsonian disorder which distinguishes itself from Parkinson's disease by frequent falls, vertical gaze paresis or delayed vertical saccades, and dysphagia. Liao et al. $(2008)^{49}$ found normal AC-cVEMP latencies in nine out of ten PSP patients. However, the amplitude of the p13-n23 complex was significantly lower in the PSP group compared to the control group. The authors concluded that the diminished vestibulo-collic reflex in the PSP group could contribute to the higher fall-rate in PSP patients.

The study performed by Pollak et al. (2009) $)^{48}$ has two main shortcomings methodologically which are, a) the age difference between the control group and the Parkinson's disease group is significantly lower in favour of the control group (i.e. in the control group $46 \pm 15$ years, and in the Parkinson's disease group $66 \pm 10$ years; $\mathrm{p}<0.001$ ), and $\mathrm{b}$ ) the level of muscle contraction of the sternocleidomastoid muscles was neither standardized nor was the raw p13-n23 amplitude corrected. The authors mentioned the first shortcoming in their discussion, but not the second. Therefore the amplitude difference between the groups is not reliable. It should be stated that the authors only concluded that the cVEMPs were abnormal if the latency was significantly prolonged or if the response was absent (i.e. amplitude differences were not an argument). Thereby amplitude differences between the groups, possibly with lower amplitudes in Parkinson's disease patients as a hallmark of a reduced vestibulocollic reflex, could be missed. The study performed by Liao et al. $(2008)^{49}$ matched the PSP patients and the correct control group concerning age, but also in this study the raw cVEMP amplitudes were neither corrected nor standardized. The authors concluded that the mean p13-n23 amplitude differed significantly between the PSP and control group; however without correct standardization or correction of the raw amplitude this difference is far less reliable than stated by the authors and could possibly be influenced by technical issues and/or patient non-compliance.

Birdane et al. (2012) ${ }^{50}$ studied AC-cVEMPs in ten patients with mild cognitive impairment, ten with mild to moderate Alzheimer's disease, and ten with moderate to severe Alzheimer's disease. Neurofibrillary tangles and amyloid plaques in the neocortex are believed to be pathognomic for Alzheimer's disease. AC-cVEMPs could be obtained bilaterally in eighteen of the thirty patients, however the amplitudes were decreased when compared to the control group and the latencies were prolonged in all of them. In eight patients (26.6 percent) only unilateral responses could be obtained and in four patients AC-cVEMPs were bilaterally absent. The p13 latency was 
significantly prolonged and the p13-n23 amplitude was significantly lower in the patient group compared to the control subjects. The level of muscle contraction of the sternocleidomastoid muscle was not standardized nor was the raw p13-n23 amplitude corrected. Therefore amplitude differences should be interpreted with caution and are less reliable than stated by the authors, possibly because cognitive problems in patients with neurodegenerative disorders can easily result in noncompliance, secondarily resulting in decreased amplitudes or absent responses. The authors concluded that the brainstem too, was affected early in the course of Alzheimer's disease and that AC-cVEMPs could be a sensitive method and helpful in the early diagnosis of Alzheimer's disease ${ }^{50}$.

In our opinion at this moment there is no place for standard VEMP testing in patients with neurodegenerative disorders. However when confronted with a patient who has a neurodegenerative disorder with complaints of dizziness, which cannot be accurately localized to either the peripheral or central neurovestibular system, VEMPs can have additional localizing value in combination with video-, or electronystagmography. VEMP testing in patients with neurodegenerative disorders, however, is important for research, because the extent of brainstem involvement can be examined during the evolution of the disease possibly giving insights into the pathophysiological evolution of the disorder.

\section{Space occupying lesions in the posterior fossa}

Vestibular schwannomas are benign tumors arising from the vestibulocochlear nerve (VIII ${ }^{\text {th }}$ cranial nerve). These tumors most often arise from the superior or inferior vestibular nerve, although they sometimes arise from the cochlear nerve. The sensitivity of abnormal ipsilesional AC-cVEMPs in vestibular schwannomas is 64 to 80 percent according to the literature ${ }^{51-61}$. The most common finding is an ipsilaterally absent AC-cVEMP which is five times more common than decreased AC-cVEMPs amplitudes $^{13}$. Most studies ${ }^{51-54,57,59-61}$ have the same methodological shortcoming that raw p13-n23 cVEMP amplitudes are not corrected (i.e. by dividing them through the rectified pre-stimulus EMG signal) or standardized, as discussed earlier. Therefore amplitude differences between groups or between sides within the same patient (i.e. when cVEMPs are measured ipsilaterally during monaural acoustic stimulation) should be cautiously interpreted.

Ocular vestibular evoked myogenic potentials elicited by bone-conducted vibration (BCV-oVEMPs) were studied recently by Iwasaki et al. $(2010)^{53}$ and Kinoshita et al. 
$(2013)^{54}$. The authors concluded that the sensitivity of BCV-oVEMPs for contralateral abnormal results was 63 percent and 86 percent, respectively ${ }^{53,54}$. Chiarovano et al. $(2014)^{51}$ also studied cVEMPs and oVEMPs elicited by both bone-, and air-conducted stimuli in patients with vestibular schwannomas. The overall sensitivity of combined $\mathrm{BCV}$ - and AC-oVEMPs in their study was 62 percent (i.e. thirty-nine of the sixtythree patients) for the detection of superior vestibular nerve dysfunction in patients with vestibular schwannomas. Some studies did not find a positive correlation between vestibular schwannoma tumor size and AC-cVEMPs ${ }^{13,55,60,61}$. However, patients with larger extracanicular vestibular schwannomas tended to have a higher chance of having abnormal AC-cVEMPs in comparison with patients with a smaller tumor size (when intracanalicular vestibular schwannomas were excluded) ${ }^{60,61}$. Patients with smaller vestibular schwannomas with a mean tumor size between 10 and 19 millimetres without evident brainstem compression could have p13 latency prolongation in ACcVEMPs, possibly due to underlying demyelination of the inferior vestibular nerve. Patients with larger extracanicular vestibular schwannomas with brainstem compression also had p13 latency prolongation in AC-cVEMPs, perhaps due to additional demyelinating lesions in the central vestibulospinal tract. So, increased latency of the AC-cVEMPs could be explained by demyelination of the peripheral and or central neurovestibular pathways ${ }^{13,60,61}$. Hu et al. (2009) ${ }^{33}$ showed that the chance of finding vestibular schwannomas with a tumor size of more than 2.5 centimetres increased when both AC-cVEMPs and calorics were absent. Brantberg $(2009)^{13}$ concluded that vestibular evoked myogenic potentials are at present not a useful screening test for vestibular schwannomas because of their limited sensitivity, but they can be used for pre-operative vestibular function assessment or for additional follow-up and monitoring in a 'watchful waiting' policy ${ }^{13}$.

After vestibular schwannomas, meningiomas are the second most common tumors in the cerebellopontine angle. Hu et al. (2009) ${ }^{33}$ described five patients with large cerebellopontine angle meningiomas (i.e. mean tumor size more than 2.5 centimetres), however cVEMPs were only measured in four patients, three with abnormal ACcVEMPs and one patient with bilaterally absent AC-cVEMPs who also had bilateral absent calorics. This is in contrast to the abnormal AC-cVEMPs and calorics in all five patients with large vestibular schwannomas. Su and Young $(2011)^{38}$ studied three patients with posterior fossa meningiomas with localized cerebellar involvement without brainstem or vestibular nerve compression. All had normal BCV-oVEMPs and $\mathrm{AC}-\mathrm{cVEMPs}{ }^{38}$. From these studies one could conclude that VEMPs are more sensitive than calorics in detecting vestibulocochlear nerve involvement due to external compression (as is the case in large cerebellopontine angle meningioma) and that VEMPs and caloric testing are equally reliable in detecting parenchymal vestibulocochlear nerve involvement in vestibular schwannomas. These results should 
be cautiously interpreted as, a) the case series only contain a very small number of patients ${ }^{33,38}$, b) there was no age-matched control group in the study by Hu et al. $(2009)^{33}$, c) the control group in the study by $\mathrm{Su}$ and Young $(2011)^{38}$ was not matched for age (i.e. the mean age of the control group was significantly lower), and d) abnormal VEMPs were defined as absent or delayed responses, so amplitude differences between the groups, possibly as a hallmark of vestibular nerve dysfunction, could possibly be missed.

Chu et al. (2006) ${ }^{62}$ described cVEMPs in three patients with epidermoid cysts in the posterior fossa. Epidermoid cysts are slowly progressive congenital tumors derived from ectopic epithelial cells during neural tube closure and are most often located in the cerebellopontine angle. One patient had cVEMPs testing prior to and after surgery. Pre-operative cVEMPs testing showed absent ipsilateral responses which normalized after surgery in concordance with the caloric testing (ipsilesional pre-operative canal paresis which was post-operatively restored to normal). The other two patients only had post-operative cVEMP testing which showed normal responses in one patient and bilaterally delayed responses in the other patient (possibly due to brainstem demyelination secondary to compression ${ }^{62}$.

In three out of four patients with cerebellar tumors (i.e. two patients had metastatic adenocarcinomas, one patient had a metastatic neuroblastoma, and one patient had a primary lymphoma) $\mathrm{Su}$ and Young $(2011)^{38}$ found abnormal BCV-oVEMPs and AC-cVEMPs (i.e. two patients had bilaterally absent BCV-oVEMPs and AC-cVEMPs, one patient had ipsilesional delayed BCV-oVEMPs and AC-cVEMPs, and one patient had bilateral normal responses). However in this study the patients with abnormal VEMPs had extended cerebellar lesions with brainstem compression and the patient with normal VEMPs had a localized cerebellar lesion without brainstem involvement ${ }^{38}$. The authors concluded that abnormal oVEMPs could differentiate between isolated cerebellar involvement (i.e. normal oVEMPs) and cerebellar lesions with adjacent brainstem involvement (i.e. abnormal oVEMPs), but these conclusions should be interpreted with caution, because of the very limited number of patients included in this series, and the lack of an age-matched control group as discussed earlier.

In our opinion VEMPs should not be used as a screenings test or in the standard workup of patients with vestibular schwannomas or other posterior fossa tumours because of their limited sensitivity. MRI with gadolinium contrast is the investigation of first choice. However when patients have contra-indications for MRI (e.g. extreme claustrophobia, and some types of pacemakers or metal cardiac valve prostheses) screening by means of VEMP testing in combination with cerebral computed 
tomography (CT) could be considered as an alternative. We agree with Brantberg $(2009)^{13}$ that VEMPs could be useful in monitoring patients with vestibular schwannomas in whom a 'watchful-waiting' policy is applied without additional therapy (e.g. no radiation or surgical therapy). Chiarovano et al. (2014) ${ }^{51}$ also concluded that the role of VEMPs in vestibular schwannomas was not primarily relevant for the diagnosis (e.g. however, some of their patients were discovered through VEMP testing), but mainly in the function testing of the vestibular nerve prior to treatment. Preoperative VEMP testing measures the residual superior and inferior vestibular nerve function, and thus can give insight into the necessity for post-treatment vestibular rehabilitation. Also, baseline VEMP measurements in radiosurgically treated vestibular schwannoma patients can be helpful in the detection of further vestibular nerve compromise when confronted with progressive decline of balance ${ }^{51}$. However more long-term prospective follow-up research is needed in large groups to ascertain the additional prognostic or follow-up value of VEMP testing after a therapeutic intervention (i.e. radiation therapy or surgery) or rehabilitation therapy.

\section{Migraine}

Liao and Young (2004) $)^{63}$ described AC-cVEMPs, electronystagmography and calorics in twenty patients with migraine with brainstem aura (formerly known as basilar-type migraine) during the headache attack. The control group in this study was not clearly defined (e.g. the mean age is not known) and the laboratory's own upper limit cVEMP values for the latencies and amplitudes were used. Therefore the comparison of the results of migraine with brainstem aura patients with the control group should be interpreted with caution. Migraine with brainstem aura is a rare form of migraine with aura symptoms and signs which seem to originate from the brainstem. During the migraine attack 30 percent of the eye-tracking tests, 45 percent of the optokinetic response tests, 55 percent of the caloric tests (five out of twenty patients had a unilateral canal paresis, four out of twenty patients had a directional preponderance, and two patients had a combination of both), and 50 percent of the AC-cVEMPs were abnormal. Most prominent were absent responses in the AC-cVEMPs; eight out of twenty patients had absent unilateral or bilateral responses followed by delayed responses (two out of twenty patients had bilaterally delayed response and one patient had a unilaterally delayed response in combination with a unilaterally absent response). When AC-cVEMPs and caloric testing results were combined 75 percent of the patients had abnormal results (four patients had abnormal AC-cVEMPS only, five patients had abnormal calorics only, and six patients had abnormal calorics and ACcVEMPs). The authors concluded that these findings were due to ischemia, attributed to hypoperfusion in the territory of the basilar artery ${ }^{63}$. However, most experts 
nowadays believe that an aura also in migraine with brainstem aura is due to cortical spreading depression in which vascular constriction is a secondary phenomenon resulting from the neuronal depression due to neurometabolic coupling, instead of the previous explanation ${ }^{64,65}$.

Vestibular migraine (VM) is an uncommon subtype of migraine with paroxysmal vertigo as the main symptom. Recently, this disorder was included in the third edition of the International Classification of Headaches Disorders (ICHD-III). The estimated prevalence of VM is 9 percent of all the migraine patients ${ }^{66,67}$. Although the pathophysiology of VM is unknown, some experts believe that this disorder shares a pathophysiological mechanism with Menière's disease (i.e. endolymphatic hydrops). Murofushi et al. (2009) ${ }^{68}$ studied eleven patients with migraine associated vertigo, eleven patients with Menière's disease, and eight control subjects. Healthy control subjects had an average negative slope concerning the p13-n23 amplitudes when acoustic tone-burst stimulation at 500 Hertz was compared with 1000 Hertz stimulation (i.e. higher amplitudes at 500 Hertz). However, patients with Menière's disease had an average positive slope when the affected ear was tested with an amplitude shift towards the 1000 Hertz. The results of the unaffected ear were similar to those of the healthy subjects. On average, the VM patients showed a very mild negative slope, however, 27 percent of the VM patients showed a positive slope with an amplitude shift towards the $1000 \mathrm{Hertz}$, comparable with the Menière's disease patients. The authors concluded that the underlying pathophysiology concerning VM is probably heterogeneous, but might share a link with Menière's disease ${ }^{68}$. The authors also concluded that the mean corrected p13-n23 amplitude of the affected side of the Menière's disease patients was significantly lower than the VM and control group at $500 \mathrm{~Hz}$ and $1000 \mathrm{~Hz}$ auditive tone-burst stimulation. However the mean age differed between groups (i.e. Menière's disease group 58.2 years, VM 41.3 years, and control group 37.3 years). Therefore, the amplitudes of the Menière's disease group cannot be reliably compared to the other groups, even though cVEMP amplitudes were corrected for contraction force. This conclusion was also drawn by Baier and Dieterich $(2009)^{69}$ from a study in sixty-three patients with VM and sixteen patients with Menière's disease. In this study 52 percent of the VM patients and 25 percent of the Menière's disease patients had abnormal AC-cVEMPs (i.e. bilaterally reduced amplitudes) which could also suggest a possible underlying pathophysiological link between these diseases ${ }^{69}$. Abnormal responses in AC-cVEMPs testing in VM patients in literature ranged from 23 to 68 percent. The most common abnormality was a reduction in amplitude with mainly preserved latencies, followed by absent responses ${ }^{69-72}$. 
Most of the studies described above share the same methodological shortcoming, i.e. that studied groups were relatively small, and that the raw unrectified cVEMP p13-n23 amplitude was neither standardized nor corrected ${ }^{63,70-72}$, as described earlier, with the exception of the studies performed by Baier and Dieterich (2009) ${ }^{69}$, and Murofushi et al. $(2009)^{68}$. Therefore amplitude differences between the groups should be interpreted with caution. The study performed by Zuniga et al. $(2012)^{72}$ tested vertical saccades in both eyes prior to oVEMP testing. The electrodes beneath the eyes were replaced if the unrectified EMG signal amplitude during vertical saccades differed by more than 25 percent. This seams an interesting method for further oVEMP standardization, but further studies on this topic have to be performed in larger groups to confirm this finding.

In our opinion nowadays standard VEMP testing in headache patients has no additional value in the individual patient, because diagnostic or therapeutic consequences are lacking. VEMP testing in different headache disorders can be important for research, e.g. further elucidating the underlying pathophysiology, as shown in Menière's disease and vestibular migraine.

\section{In summary}

VEMP abnormalities in central neurological disorders may be seen rather often, but are not disease specific and usually do not give any information about the underlying aetiology. In contrast, VEMPs may give important localising information (table 1). In peripheral labyrinthine disorders like Menière's disease or labyrinthine infarction one may expect a reduction in amplitude or even disappearance of the ipsilateral cVEMP response and/or contralateral oVEMP response. In contrast to this, increased amplitudes and lower thresholds of the contralateral oVEMP and of the ipsilateral cVEMP may be found in the superior semicircular canal dehiscence syndrome, however, amplitude differences in oVEMPs are more pronounced. The cause of this phenomenon is supposed to be due to an increased sound and pressure sensitivity of the ipsilateral labyrinth in comparison to the contralateral side $e^{73,74}$. Delayed latencies are in general considered to be of retro-labyrinthine origin. As discussed elsewhere in this paper, VEMP changes are obviously not only affected by peripheral labyrinthine end-organ damage or vestibular nerve pathology, but also central neurological disorders can affect both cVEMP and oVEMP results. So VEMP testing done for suspected peripheral vestibular disorders should therefore be interpreted with great care when central neurological disorders, that could also possibly affect the VEMP results, are present and vice versa. 
Due to the physiology as described elsewhere in this paper, abnormalities limited to the inferior or superior vestibular nerve will result in either ipsilateral cVEMP or contralateral oVEMP abnormalities, respectively. This may be of help in differentiating these type of lesions. Most commonly decreased or abolished responses are found, as for example in vestibular schwannomas or vestibular neuritis ${ }^{3}$. Less commonly, prolongation of latencies can also occur, due to focal demyelination, as for example can be seen in external vestibular nerve compression due to a cerebellopontine angle meningioma ${ }^{38}$.

Tabel 1. Expected VEMP abnormalities at different anatomical localizations.

\begin{tabular}{|c|c|c|c|}
\hline \multirow{2}{*}{\multicolumn{2}{|c|}{$\begin{array}{l}\text { Anatomical } \\
\text { localization }\end{array}$}} & \multicolumn{2}{|l|}{ Expected abnormalities } \\
\hline & & oVEMP & cVEMP \\
\hline \multicolumn{2}{|c|}{ Peripheral labyrinth } & $\begin{array}{l}\text { Contralesionally absent responses } \\
\text { or low amplitudes. }\end{array}$ & $\begin{array}{l}\text { Ipsilesionally absent responses or } \\
\text { low amplitudes. }\end{array}$ \\
\hline \multicolumn{2}{|c|}{ Vestibular nerve } & $\begin{array}{l}\text { Contralesionally absent responses } \\
\text { or low amplitudes >> latency } \\
\text { prolongation (due to external } \\
\text { vestibular nerve compression). }\end{array}$ & $\begin{array}{l}\text { Ipsilesionally absent responses or } \\
\text { low amplitudes >> latency } \\
\text { prolongation (due to external } \\
\text { vestibular nerve compression). }\end{array}$ \\
\hline \multirow[t]{2}{*}{ Brainstem } & Pontine & $\begin{array}{l}\text { Contralesionally or bilaterally } \\
\text { absent responses, low amplitudes, } \\
\text { or latency prolongation are all } \\
\text { possible. }\end{array}$ & $\begin{array}{l}\text { Bilaterally normal responses are } \\
\text { expected. However, with more } \\
\text { caudal disease progression absent } \\
\text { responses, low amplitudes, or } \\
\text { latency prolongation, mostly } \\
\text { ipsilesional or bilateral, are all } \\
\text { possible. }\end{array}$ \\
\hline & Medulla & $\begin{array}{l}\text { Bilaterally normal responses are } \\
\text { expected. However, with more } \\
\text { rostral disease progression absent } \\
\text { responses, low amplitudes, or } \\
\text { latency prolongation mostly } \\
\text { contralesional or bilateral are all } \\
\text { possible. }\end{array}$ & $\begin{array}{l}\text { Ipsilesionally or bilaterally absent } \\
\text { responses, low amplitudes, or } \\
\text { latency prolongation are all } \\
\text { possible. }\end{array}$ \\
\hline \multicolumn{2}{|l|}{ Cerebellar } & $\begin{array}{l}\text { Normal responses or possibly } \\
\text { contralesionally absent responses } \\
\text { or amplitude differences (both } \\
\text { decrease and increase are possible). }\end{array}$ & $\begin{array}{l}\text { Normal responses or possibly } \\
\text { ipsilesionally absent responses or } \\
\text { amplitude differences (both } \\
\text { decrease and increase are possible). }\end{array}$ \\
\hline
\end{tabular}


In brainstem and cerebellar disorders, abnormal VEMPs may be found due to demyelination or axonal degeneration. Obviously, significant prolongation of response latencies is associated most commonly with demyelination and decreased response amplitudes with conduction block or axonal damage. As can be concluded from figure 1 for instance, brainstem lesions rostral to the decussation of the ascending medial longitudinal fasciculus (MLF) will most likely result in ipsilesionally absent oVEMPs, when concerning a unilateral lesion with normal cVEMPs (e.g. in case of internuclear ophthalmoplegia ${ }^{25,35}$. However, lesions caudal to the decussation of the ascending MLF and rostral to the vestibular nuclei will result in absent oVEMPs contralateral to the lesions, however, with bilaterally preserved and normal cVEMPs. In a similar way careful interpretation of VEMP abnormalities would be expected to lead to localization. The literature concerning VEMP abnormalities in localized cerebellar lesions is conflicting, because some studies could not show significant VEMP changes in localized cerebellar disorders without adjacent brainstem involvement, as discussed earlier ${ }^{37,38}$.

VEMPs may be easily performed by well-trained neurophysiological technicians, are well tolerated by patients, and therefore readily applicable in the outpatient clinic. Methodology and a correct control group are very important as stated before. Recently international guidelines were published concerning the clinical application as well as standardization of $\mathrm{cVEMPs}^{2}$ in research as well as the clinical testing. In addition, we would like to advice the acquisition of data from a healthy age-matched control group, particularly as amplitudes in acoustically elicited VEMPs decrease at advanced ages 2,29,30. $^{2}$.

Most studies, as discussed in the previous sections, share the same methodological and technical concerns which are, a) that the control group sometimes is not correctly matched for age to the patient group, b) that most case-series do not have a control group at all whereby laboratory's own reference values were used, c) that the VEMP amplitudes mostly are not standardized for force of contraction, nor corrected by the raw mean pre-stimulus EMG background signal, and d) that most studies consisted of small sized case-series or case-control studies with a few exceptions (mostly concerning diagnostic studies in Multiple Sclerosis, vestibular schwannomas, and vertebrobasilar stroke). Therefore the diagnostic level of evidence for all studies according to the Oxford Centre of Evidence-Based Medicine mainly consists of level 4 evidence with a few exceptions, reaching a maximum level of $2 b$ evidence, whereby the total diagnostic evidence is very limited concerning VEMPs in central neurological disorders. 
In our opinion the primary indication for VEMP testing should be in patients in whom the neurovestibular complaints cannot reliably be localized to the central or peripheral neurovestibular system clinically nor with (video)nystagmography or brain MRI. Also VEMP testing can help in confirming central nervous system involvement in patients with vestibular complaints with normal brain MRI. At the moment VEMPs are more important in research than in clinical practice in most patients with central nervous system disorders. However, VEMPs can have additional diagnostic value in a selected group of patients, as discussed earlier. Suggestions for future research have been made in the previous sections. 


\section{References}

1. Curthoys, I. S. A critical review of the neurophysiological evidence underlying clinical vestibular testing using sound, vibration and galvanic stimuli. Clinical Neurophysiology 121, 132-144 (2010).

2. Papathanasiou, E. S., Murofushi, T., Akin, F. W. \& Colebatch, J. G. International guidelines for the clinical application of cervical vestibular evoked myogenic potentials: an expert consensus report. Clin. Neurophysiol. 125, 658-66 (2014).

3. Rosengren, S. M., Welgampola, M. S. \& Colebatch, J. G. Vestibular evoked myogenic potentials: Past, present and future. Clin. Neurophysiol. 121, 636-651 (2010).

4. Rosengren, S. \& Kingma, H. New perspectives on vestibular evoked myogenic potentials. Curr. Opin. Neurol. 26, 74-80 (2013).

5. Welgampola, M. S., Welgampola, M. S., Colebatch, J. G. \& Colebatch, J. G. Characteristics and clinical applications of vestibular-evoked myogenic potentials. Neurology 64, 1682-1688 (2005).

6. Eleftheriadou, A. \& Koudounarakis, E. Vestibular-evoked myogenic potentials eliciting: An overview. European Archives of Oto-Rhino-Laryngology 268, 331-339 (2011).

7. Colebatch, J. G. Sound conclusions? Clin. Neurophysiol. 121, 124-126 (2010).

8. Curthoys, I. S. A balanced view of the evidence leads to sound conclusions. A reply to J.G. Colebatch 'Sound conclusions?'. Clin. Neurophysiol. 121, 977-8 (2010).

9. Papathanasiou, E. S. Ocular vestibular evoked myogenic potentials (OVEMPs): saccule or utricle? Clin. Neurophysiol. 123, 216 (2012).

10. Papathanasiou, E. S. \& Papacostas, S. S. Vestibular evoked myogenic potentials: the fuzzy picture of different stimulation types is beginning to come into focus. Clin. Neurophysiol. 124, 1926-7 (2013).

11. Todd, N. The ocular vestibular evoked myogenic potential (OVEMP), ten years old. Clin. Neurophysiol. 125, 439-41 (2014).

12. Walther, L. E., Rogowski, M., Hörmann, K., Schaaf, H. \& Löhler, J. Ocular vestibular evoked myogenic potentials to air conduction (AC oVEMP): useful in clinical practice? Otolaryngol. Pol. 65, 333-8

13. Brantberg, K. Vestibular evoked myogenic potentials (VEMPs): Usefulness in clinical neurotology. Seminars in Neurology 29, 541-547 (2009).

14. Compston, A. \& Coles, A. Multiple sclerosis. The Lancet 372, 1502-1517 (2008).

15. Alpini, D. et al. Vestibular evoked myogenic potentials in multiple sclerosis: clinical and imaging correlations. Mult. Scler. 10, 316-21 (2004).

16. Bandini, F. et al. The diagnostic value of vestibular evoked myogenic potentials in multiple sclerosis. J. Neurol. 251, 617-21 (2004).

17. Escorihuela, G., Llópez, C., AM, O. \& Marco, A. Hallazgos de potenciales vestibulares miogénicos evocados en la esclerosis multiple. Acta Otorrinolaringol Esp 64, 352-358 (2013).

18. Güven, H. et al. Vestibular-evoked myogenic potentials, clinical evaluation, and imaging findings in multiple sclerosis. Neurol. Sci. 35, 221-226 (2014).

19. Gazioglu, S. \& Boz, C. Ocular and cervical vestibular evoked myogenic potentials in multiple sclerosis patients. Clin. Neurophysiol. 123, 1872-1879 (2012).

20. Harirchian, M. H. et al. Vestibular evoked myogenic potential for diagnoses of multiple sclerosis: is it beneficial? Med. Glas. (Zenica). 10, 321-6 (2013).

21. Ivanković, A. et al. Auditory evoked potentials and vestibular evoked myogenic potentials in evaluation of brainstem lesions in multiple sclerosis. J. Neurol. Sci. 328, 24-27 (2013).

22. Patkó, T., Simó, M. \& Arányi, Z. Vestibular click-evoked myogenic potentials: sensitivity and factors determining abnormality in patients with multiple sclerosis. Mult. Scler. J. 13, 193-198 (2007).

23. Sartucci, F. \& Logi, F. Vestibular-evoked myogenic potentials: a method to assess vestibulo-spinal conduction in multiple sclerosis patients. Brain Res. Bull. 59, 59-63 (2002).

24. Versino, M. et al. Vestibular evoked myogenic potentials in multiple sclerosis patients. Clin. Neurophysiol. 113, 1464-9 (2002).

25. Rosengren, S. M. \& Colebatch, J. G. Ocular vestibular evoked myogenic potentials are abnormal in internuclear ophthalmoplegia. Clin. Neurophysiol. 122, 1264-1267 (2011).

26. Vanspauwen, R., Wuyts, F. L. \& Van De Heyning, P. H. Validity of a new feedback method for the VEMP test. Acta Otolaryngol. 126, 796-800 (2006). 
27. Vanspauwen, R., Wuyts, F. L. \& Van De Heyning, P. H. Vestibular evoked myogenic potentials: Test-retest reliability and normative values obtained with a feedback method for the sternocleidomastoid muscle contraction. J. Vestib. Res. Equilib. Orientat. 19, 127-135 (2009).

28. Colebatch, J. G., Halmagyi, G. M. \& Skuse, N. F. Myogenic potentials generated by a click-evoked vestibulocollic reflex. J. Neurol. Neurosurg. Psychiatry 57, 190-197 (1994).

29. Colebatch, J. G., Govender, S. \& Rosengren, S. M. Two distinct patterns of VEMP changes with age. Clin. Neurophysiol. 124, 2066-8 (2013).

30. Rosengren, S. M., Govender, S. \& Colebatch, J. G. Ocular and cervical vestibular evoked myogenic potentials produced by air- and bone-conducted stimuli: comparative properties and effects of age. Clin. Neurophysiol. 122, 2282-9 (2011).

31. Nouh, A., Remke, J. \& Ruland, S. Ischemic posterior circulation stroke: A review of anatomy, clinical presentations, diagnosis, and current management. Frontiers in Neurology 5 APR, (2014).

32. Ahn, B. H., Kim, H. A., Yi, H. A., Oh, S. Y. \& Lee, H. Abnormal cervical vestibular-evoked myogenic potential in anterior inferior cerebellar artery territory infarction: Frequency, pattern, and a determinant. J. Neurol. Sci. 307, 114-119 (2011).

33. Hu, Y.-F., Cheng, P.-W. \& Young, Y.-H. Comparison of vestibular function between large cerebellopontine angle meningioma and schwannoma. Acta Otolaryngol. 129, 161-165 (2009).

34. Choi, S. Y., Lee, S. H., Kim, H. J. \& Kim, J. S. Impaired modulation of the otolithic function in acute unilateral cerebellar infarction. Cerebellum 13, 362-371 (2014).

35. Kim, H.-J., Lee, J. H. \& Kim, J.-S. Ocular vestibular evoked myogenic potentials to head tap and cervical vestibular evoked myogenic potentials to air-conducted sounds in isolated internuclear ophthalmoplegia. Clin. Neurophysiol. 125, 1042-7 (2014).

36. Oh, S. Y. et al. Ocular vestibular evoked myogenic potentials induced by air-conducted sound in patients with acute brainstem lesions. Clin. Neurophysiol. 124, 770-778 (2013).

37. Pollak, L., Kushnir, M. \& Stryjer, R. Diagnostic value of vestibular evoked myogenic potentials in cerebellar and lower-brainstem strokes. Neurophysiol. Clin. 36, 227-33 (2006).

38. Su, C. H. \& Young, Y. H. Differentiating cerebellar and brainstem lesions with ocular vestibularevoked myogenic potential test. Eur. Arch. Oto-Rhino-Laryngology 268, 923-930 (2011).

39. Tseng, C.-L. \& Young, Y.-H. Topographical correlations of lateral medullary infarction with caloricand vestibular-evoked myogenic potential results. Eur. Arch. Otorhinolaryngol. 267, 191-5 (2010).

40. Weng, Y.-C. \& Young, Y.-H. Mapping affected territory of anterior/posterior inferior cerebellar artery infarction using a vestibular test battery. Acta Otolaryngol. 134, 268-274 (2014).

41. Miwa, T., Minoda, R. \& Matsuyoshi, H. Vestibular function in superficial siderosis. BMC Ear, Nose Throat Disord. 13, (2013).

42. Ushio, M., Iwasaki, S., Sugasawa, K. \& Murofushi, T. Superficial siderosis causing retrolabyrinthine involvement in both cochlear and vestibular branches of the eighth cranial nerve. Acta Otolaryngol. 126, 997-1000 (2006).

43. Chen, C.-H. \& Young, Y.-H. Vestibular evoked myogenic potentials in brainstem stroke. Laryngoscope 113, 990-3 (2003).

44. Kattah, J. C., Talkad, A. V., Wang, D. Z., Hsieh, Y. H. \& Newman-Toker, D. E. HINTS to diagnose stroke in the acute vestibular syndrome: Three-step bedside oculomotor examination more sensitive than early MRI diffusion-weighted imaging. Stroke 40, 3504-3510 (2009).

45. Etgen, T. et al. Detection of acute brainstem infarction by using DWI/MRI. Eur. Neurol. 52, 145-50 (2004).

46. Newman-Toker, D. E. et al. HINTS outperforms ABCD2 to screen for stroke in acute continuous vertigo and dizziness. Acad. Emerg. Med. 20, 987-996 (2013).

47. Takegoshi, H. \& Murofushi, T. Vestibular evoked myogenic potentials in patients with spinocerebellar degeneration. Acta Otolaryngol. 120, 821-4 (2000).

48. Pollak, L., Prohorov, T., Kushnir, M. \& Rabey, M. Vestibulocervical reflexes in idiopathic Parkinson disease. Neurophysiol. Clin. 39, 235-240 (2009).

49. Liao, K. et al. Why do patients with PSP fall? Evidence for abnormal otolith responses. Neurology 70, 802-809 (2008).

50. Birdane, L., Incesulu, A., Gurbuz, M. K. \& Ozbabalik, D. Sacculocolic reflex in patients with dementia: is it possible to use it for early diagnosis? Neurol. Sci. 33, 17-21 (2012). 
51. Chiarovano, E., Darlington, C., Vidal, P.-P., Lamas, G. \& de Waele, C. The role of cervical and ocular vestibular evoked myogenic potentials in the assessment of patients with vestibular schwannomas. PLoS One 9, e105026 (2014).

52. Day, A.-S., Wang, C.-T., Chen, C.-N. \& Young, Y.-H. Correlating the cochleovestibular deficits with tumor size of acoustic neuroma. Acta Otolaryngol. 128, 756-760 (2008).

53. Iwasaki, S. et al. Ocular vestibular evoked myogenic potentials to bone-conducted vibration in vestibular schwannomas. Otol. Neurotol. 31, 147-52 (2010).

54. Kinoshita, M. et al. Ocular vestibular evoked myogenic potentials in response to air-conducted sound and bone-conducted vibration in vestibular schwannoma. Otol. Neurotol. 34, 1342-8 (2013).

55. Murofushi, T., Matsuzaki, M. \& Mizuno, M. Vestibular evoked myogenic potentials in patients with acoustic neuromas. Arch. Otolaryngol. Head. Neck Surg. 124, 509-12 (1998).

56. Murofushi, T., Shimizu, K., Takegoshi, H. \& Cheng, P. W. Diagnostic value of prolonged latencies in the vestibular evoked myogenic potential. Arch. Otolaryngol. Head. Neck Surg. 127, 1069-1072 (2001).

57. Patko, T., Vidal, P.-P., Vibert, N., Tran Ba Huy, P. \& de Waele, C. Vestibular evoked myogenic potentials in patients suffering from an unilateral acoustic neuroma: a study of 170 patients. Clin. Neurophysiol. 114, 1344-50 (2003).

58. Suzuki, M. et al. Analysis of Vestibular Testing in Patients With Vestibular Schwannoma Based on the Nerve of Origin, the Localization, and the Size of the Tumor. Otol. Neurotol. 29, 1029-1033 (2008).

59. Takeichi, N., Sakamoto, T., Fukuda, S. \& Inuyama, Y. Vestibular evoked myogenic potential (VEMP) in patients with acoustic neuromas. Auris. Nasus. Larynx 28 Suppl, S39-41 (2001).

60. Ushio, M. et al. The diagnostic value of vestibular-evoked myogenic potential in patients with vestibular schwannoma. Clin. Neurophysiol. 120, 1149-1153 (2009).

61. Ushio, M. et al. Is the nerve origin of the vestibular schwannoma correlated with vestibular evoked myogenic potential, caloric test, and auditory brainstem response? Acta Otolaryngol. 129, 1095-1100 (2009).

62. Chu, C.-K., Tseng, H.-M. \& Young, Y.-H. Clinical presentation of posterior fossa epidermoid cysts. Eur. Arch. Oto-Rhino-Laryngology 263, 548-551 (2006).

63. Liao, L.-J. \& Young, Y.-H. Vestibular Evoked Myogenic Potentials in Basilar Artery Migraine. Laryngoscope 114, 1305-1309 (2004).

64. Cutrer, F. M. \& Huerter, K. Migraine aura. Neurologist 13, 118-25 (2007).

65. Richter, F., Bauer, R., Lehmenkühler, A. \& Schaible, H.-G. Spreading Depression in the Brainstem of the Adult Rat: Electrophysiological Parameters and Influences on Regional Brainstem Blood Flow. J. Cereb. Blood Flow Metab. 28, 984-994 (2008).

66. Lempert, T. Vestibular Migraine. Semin. Neurol. 33, 212-218 (2013).

67. Lempert, T. et al. Vestibular migraine: Diagnostic criteria. J. Vestib. Res. Equilib. Orientat. 22, 167-172 (2012).

68. Murofushi, T., Ozeki, H., Inoue, A. \& Sakata, A. Does Migraine-Associated Vertigo Share a Common Pathophysiology With Meniere's Disease? Study With Vestibular-Evoked Myogenic Potential. Cephalalgia 29, 1259-1266 (2009).

69. Baier, B. \& Dieterich, M. Vestibular-Evoked Myogenic Potentials in 'Vestibular Migraine' and Menière's Disease. Ann. N. Y. Acad. Sci. 1164, 324-327 (2009).

70. Boldingh, M. I., Ljøstad, U., Mygland, A. \& Monstad, P. Vestibular sensitivity in vestibular migraine: VEMPs and motion sickness susceptibility. Cephalalgia 31, 1211-1219 (2011).

71. Hong, S. M., Kim, S. K., Park, C. H. \& Lee, J. H. Vestibular-evoked myogenic potentials in migrainous vertigo. Otolaryngol. Head. Neck Surg. 144, 284-287 (2011).

72. Zuniga, M. G., Janky, K. L., Schubert, M. C. \& Carey, J. P. Can Vestibular-Evoked Myogenic Potentials Help Differentiate Meniere Disease from Vestibular Migraine? Otolaryngol. -- Head Neck Surg. 146, 788-796 (2012).

73. Yew, A. et al. Characteristics and management of superior semicircular canal dehiscence. J. Neurol. Surg. B. Skull Base 73, 365-70 (2012).

74. Zuniga, M. \& Janky, K. Ocular vs. cervical VEMPs in the diagnosis of superior semicircular canal dehiscence syndrome. Otol. Neurotol. Off. Publ. Am. Otol. Soc. Am. Neurotol. Soc. [and] Eur. Acad. Otol. Neurotol. Neurotol. 34, 121-126 (2013). 


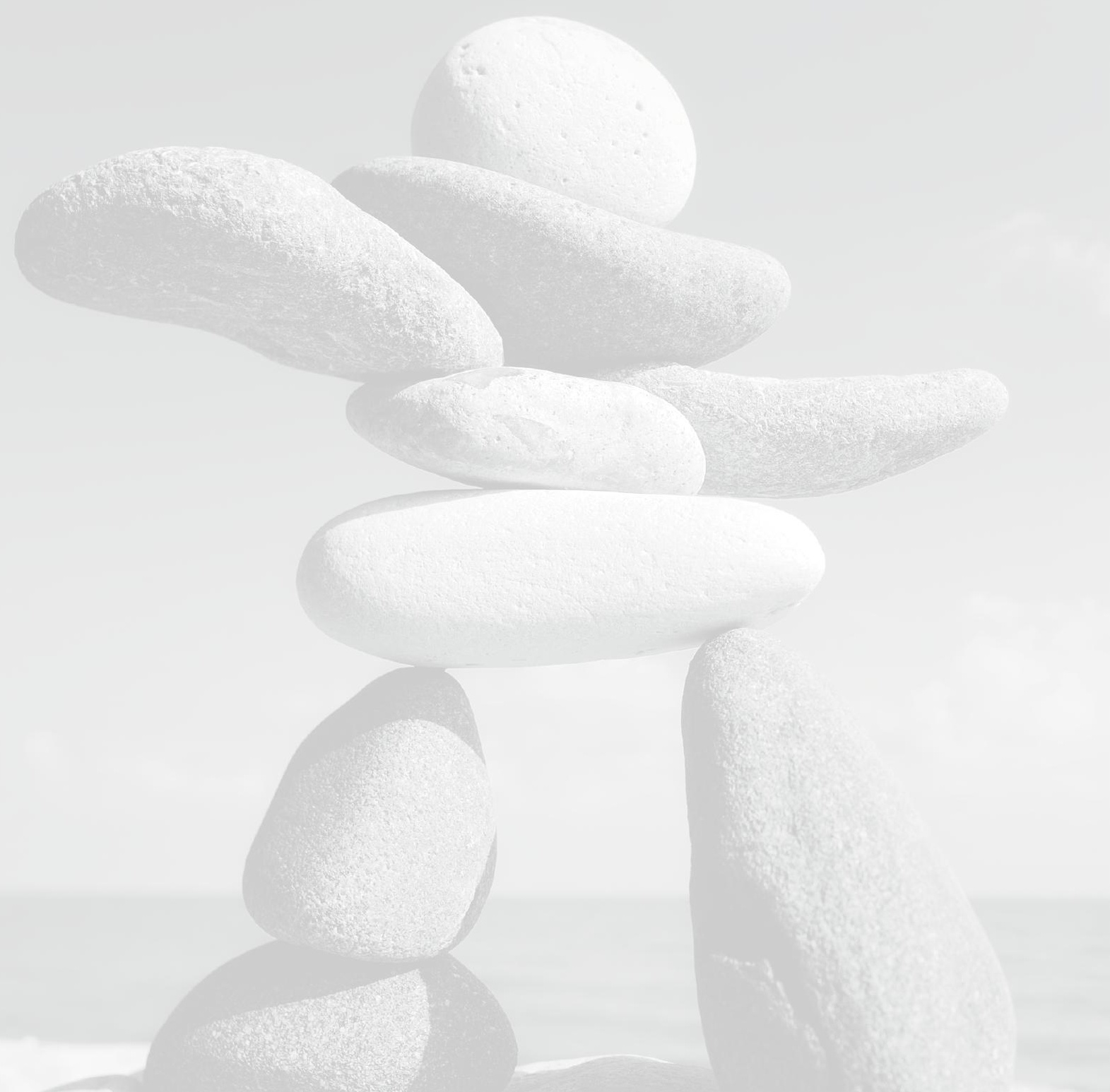




\section{Chapter 3}

Ocular and cervical vestibular evoked myogenic potentials (VEMPs) in healthy volunteers:

The intra-, interobserver, and the test re-test reliability

J. Venhovens, J. Meulstee, W.I.M. Verhagen.

J. Vestib. Res. 25, 161-7 (2015) 


\section{Abstract}

\section{Objective}

The aims of the study are to determine the intra-, interobserver, and the test retest reliability of the cervical and ocular vestibular evoked myogenic potentials (VEMPs).

\section{Methods}

Twenty healthy subjects underwent acoustically and forehead tap elicited cervical and ocular VEMPs. The measurements were repeated one week later.

\section{Results}

The intra- and interobserver reliability of both ocular and cervical VEMPs is excellent. The test re-test reliability of the raw p13n23 peak-to-peak amplitudes of the cervical VEMPs is excellent (ICC: $0.76,0.87$ ) and the p13 latencies show a good reliability (ICC: $0.56,0.73$ ). The raw $\mathrm{n} 1 \mathrm{p} 1$ peak-to-peak amplitudes of the ocular VEMPs show a fair-to-good test re-test reliability (ICC: $0.51,0.64$ ) and the $\mathrm{n} 1$ and $\mathrm{p} 1$ latencies show a poor reliability (ICC: $-0.17 \leq \mathrm{x} \leq 0.44$ ).

\section{Conclusions}

The intra- and interobserver reliability of the cervical and ocular VEMPs is excellent. The raw ocular and cervical VEMP peak-to-peak amplitudes are the most reliable parameters, followed by the cervical VEMP latencies. The ocular VEMP latencies shows a poor test re-test reliability. The individual VEMP results, however, remained within normal limits despite the test re-test variability. 


\section{Introduction}

The classical detailed neurophysiological examination of the vestibular system can be time consuming, uncomfortable for the patient, and is in most laboratories limited to the examination of the horizontal semicircular canal and the superior vestibular nerve by means of calorics and rotary chair testing in combination with electro- or videonystagmography. Vestibular evoked myogenic potentials (VEMPs), however, emerged as a test suitable for detailed neurophysiological examination of both the peripheral and the central vestibular system in the last two decades, and can be easily performed by well-trained technicians. VEMP responses can be divided into a cervical and an ocular response; which can be elicited by short intense auditory stimuli (i.e. tone bursts or clicks), bone-conducted vibration, forehead taps, and galvanic stimulation $^{1-6}$. We refer to the review by Brantberg $(2009)^{7}$ for a detailed overview concerning VEMP abnormalities in peripheral vestibular disorders, and to the recent review article by Venhovens et al. (2016) for an overview concerning VEMP abnormalities resulting from central nervous system disorders.

The cervical VEMPs can be recorded by placing active surface electrodes over the middle upper part of both sternocleidomastoid (SCM) muscle bellies with a reference electrode on the sternal manubrium. The SCM muscles, however, first have to be tightened before cervical VEMPs can be obtained. This can be done by rotating the head to the contralateral side in monaural stimulation (in auditory stimuli such as short tone bursts or clicks) or by flexing the neck (which can be used in all the types of stimuli and especially when bilateral responses need to be obtained simultaneously, for instance when tap stimulation is applied at $\mathrm{Fz}$ ). The primary end-organ responsible for generating cervical VEMPs is the sacculus. The signal is thereafter transmitted to the vestibular nuclei through the inferior vestibular nerve, and subsequently to the ipsilateral sternocleidomastoid muscle through the medial vestibulospinal tract, the cervical motor neurons, and the accessory nerve. The cervical VEMPs represent an inhibitory motor response in the ipsilateral sternocleidomastoid muscle, and measure the integrity of the vestibulo-collic reflex ${ }^{2-5,8,9}$.

The ocular VEMPs can be recorded by placing active surface electrodes beneath the infraorbital margin and reference electrodes 1 to 2 centimetres below them. The ocular VEMPs measure the integrity of the vestibulo-ocular reflex and represent an excitatory motor response of the inferior oblique muscles, mainly in the contralateral side in reference to the stimulated ear. The best method of measuring these ocular VEMP responses is to have the subject maintain an upward gaze during the stimulation. The exact otolith end-organ for generating the ocular VEMPs is uncertain (i.e. if it is the sacculus, the utriculus, or a combination of the both) and this matter is extensively 
debated in the literature. However, there is consensus that the responsible afferents travel through the superior division of the vestibular nerve to the vestibular nuclei, and subsequently to the contralateral oculomotor nerve through the medial longitudinal fasciculus and the oculomotor nucleus before reaching the contralateral inferior oblique muscle $e^{2-6,8-14}$.

The primary aim of our study is to determine the intra- and interobserver reliability concerning the assessments of individual ocular and cervical VEMP recordings by three different experienced clinical neurophysiologists. We hypothesize that the reliability within and between observers needs to be good to excellent in order for the VEMPs to be a reliable clinical tool; to our knowledge this is the first study on this important aspect of clinical VEMP judgement. The secondary aim of our study is to assess the test re-test reliability for ocular and cervical VEMPs by both acoustic and bone conducted stimuli; to our knowledge, only one prior study has been published concerning this reliability in ocular VEMPs ${ }^{15}$ and a few studies concerning the cervical VEMPs ${ }^{15-22}$, some of which show conflicting results as will be discussed later on. We calculated the reliability between the two consecutive tests, which were separated by one week, and compared the outcomes with the existing literature.

\section{Methodology}

\section{Study Participants}

Twenty volunteers, none of whom had a relevant medical history (they did not have neurological, otological, and/or ophthalmological diseases or complaints), were included. The study was approved by the local medical ethical committee and all volunteers signed an informed consent. Twenty participants (mean age 24, range 2129 years old, $8 \mathrm{men}$ ) were included in the study.

\section{Tests and procedure}

The VEMPs were recorded using $\mathrm{Ag} / \mathrm{AgCl}$ surface electrodes; abrasive gel was applied on the recording sites before the surface electrodes were placed to assure that the impedance levels were well below the maximum of $2 \mathrm{k} \Omega$. The difference between the side-to-side impedance levels had to be less than $1 \mathrm{k} \Omega$ to assure that the possible amplitude differences between both sides could not be attributed to differences in the background noise. The active electrodes for the cervical VEMP were applied on symmetrical sites over the upper middle part of the muscle belly of the sternocleidomastoid muscles and the reference electrode was placed on the sternal manubrium. The active electrodes for the ocular VEMP were symmetrically placed over the middle part of the lower eyelids, on top of the inferior orbital edges, and the 
reference electrodes were placed 2 centimeters below them. The ground electrode was placed at Cz. All subjects had a subjective hearing threshold of less than $20 \mathrm{~dB}$ for acoustic $500 \mathrm{~Hz}$ tone bursts.

The cervical and ocular VEMPs were measured separately and were recorded with a Medelec Synergy T-EP5 EMG/EP machine (Oxford Medical Instruments, Surrey, England). All evoked responses were amplified, band-pass filtered $(10-1000 \mathrm{~Hz})$, notch-filtered $(50 \mathrm{~Hz})$, and averaged. We did not apply artifact rejection during the recording and did not perform electromyographic analysis on the pre-stimulus signal. The cervical and ocular VEMPs were elicited by: a) forehead taps at Fz with a triggered reflex hammer (Aesculap, type AC012C, Oxford Medical Instruments, England), and subsequently b) acoustically by means of short tone bursts with an acoustically shielded headphone (TDH49P acoustically shielded headphone, Oxford Medical Instruments, England, rise/fall time: $1 \mathrm{~ms}$, plateau time $2 \mathrm{~ms}$, repetition rate $5 \mathrm{~Hz}$, frequency $500 \mathrm{~Hz}$, $100 \mathrm{~dB}$ NHL). For each trial, twenty forehead taps at Fz with a tap frequency of $1 \mathrm{~Hz}$ (in tap evoked VEMPs) or two hundred acoustic stimuli were averaged. We checked three trials for reproducibility.

During the cervical VEMP measurements, subjects were asked to sit upright and push their chins downward against an inflated blood pressure cuff (inflated to $60 \mathrm{mmHg}$ ) as proposed by Vanspauwen et al. (2006) ${ }^{20,21}$. A target pressure of $80 \mathrm{~mm} \mathrm{Hg}$ was chosen with a maximal variance of $2 \mathrm{mmHg}$ above or below this target pressure, as was visually monitored by both the examiner and the subject using the manometer. We interrupted the VEMP measurements every 30 seconds of contraction, with a pause of 30 seconds, to minimize the effects of muscle strain ${ }^{23}$. The peak latencies (in milliseconds, ms) of the $\mathrm{p} 13$ and $\mathrm{n} 23$ were measured, as well as the $\mathrm{p} 13-\mathrm{n} 23$ peakto-peak amplitude (in microvolts, $\mathrm{mcV}$ ).

During the ocular VEMP measurements, subjects were asked to sit upright and to look upwards at a fixed target (upward eye deviation of about 30 degrees). The peak latencies (in milliseconds, $\mathrm{ms}$ ) of the $\mathrm{n} 1, \mathrm{p} 1, \mathrm{n} 2$, and $\mathrm{p} 2$ were measured, as were the n1-p1 and n2-p2 peak-to-peak amplitudes (in microvolts, mcV).

The four VEMP tests (forehead tap and acoustically evoked cervical and ocular VEMPs) were performed at baseline in the subjects and all measurements were repeated one week later.

\section{Statistical analysis}

The three experienced clinical neurophysiologists (JV, WV, and JM) had a consensus meeting prior to the start of the study, where the local guidelines concerning the ocular and cervical VEMP assessment were run through. The three clinical neurophysiologists received the individual unmarked VEMP traces of all the subjects in print and in duplicate (both the test and re-test traces), and they were asked to assess al traces for reproducibility and to place the markers on the prints. Afterwards, the corresponding 
latencies and amplitudes of all the traces were calculated by the clinical neurophysiological research assistant and were entered into the SPSS 21.0 statistical database (by Mrs. P. Kap, SPSS software version 21.0, SPSS inc., USA). A significance level of 5 percent was adopted for each analysis.

The intra- and interobserver reliability was assessed by the intraclass correlation coefficient (ICC); a two way random effect model with absolute agreement and average measures was used for analysis. The same ICC ranking as in the other recent literature ${ }^{15,16,18,21,22}$ was adopted: ICC values above 0.75 represent excellent reliability, values between 0.4 and 0.75 represent fair-to-good reliability, and values below 0.4 represent poor reliability. The intraclass correlation coefficient is considered to be the key statistical indicator of relative reliability ${ }^{21}$.

The mean latencies (p13, n23, n1, p1, n2, and p2) and mean amplitudes (p13-n23, $\mathrm{n} 1-\mathrm{p} 1$, and $\mathrm{n} 2-\mathrm{p} 2)$ for both the test and re-test were calculated by averaging the results of all three clinical neurophysiologists. The ICC two way random effect model with absolute agreement and average measures was also used for the test re-test reliability analysis, and the ICC ranking that was described above was adopted.

\section{Results}

We refer to table 1 and 2 for the intra- and interobserver ICC values, for all three clinical neurophysiologists (WV, JV, and JM), for both the acoustically and forehead tap evoked cervical and ocular VEMPs. The overall reliability between the observers (interobserver reliability) is excellent for almost all ocular and cervical VEMP parameters with exception of the $\mathrm{p} 2$ latency reliability in the forehead tap evoked ocular VEMPs, which fluctuates between good and excellent. The overall intraobserver reliability too is excellent for most parameters, however particularly the $\mathrm{n} 2$ and $\mathrm{p} 2$ forehead tap evoked ocular VEMP latencies fluctuate between poor, fair-to-good, and excellent reliability between the different clinical neurophysiologists.

We refer to table 3 for the test re-test results concerning the acoustically and forehead tap evoked cervical and ocular VEMPs. The ICC was calculated to evaluate the test re-test reliability of different VEMP parameters. The test re-test reliability of the p13 latency in both acoustically and forehead tap evoked cervical VEMPs is overall good, with the p13n23 peak-to-peak amplitude having an excellent reliability in both. The overall test re-test reliability for both the acoustically and forehead tap evoked ocular VEMPs was poor concerning the latencies, fair-to-good for the acoustically elicited amplitudes, and good for forehead tap elicited amplitudes. 


\section{Table 1.}

Intra- and interobserver reliability concerning the acoustically evoked VEMP test and re-test assessments done by three different experienced clinical neurophysiologists (WV, JV, and JM) and analysed by the intraclass correlation coefficient (ICC, two way random effect model, average measures, and absolute agreement). We refer to the text for further explanation.

\begin{tabular}{|c|c|c|c|c|c|c|c|c|}
\hline & \multicolumn{4}{|c|}{ Acoustically evoked VEMPs (test) } & \multicolumn{4}{|c|}{$\begin{array}{c}\text { Acoustically evoked VEMPs } \\
\text { (re-test) }\end{array}$} \\
\hline & $\mathrm{WV}^{*}$ & $\mathbf{J V}^{*}$ & $\mathrm{JM}^{*}$ & INTER** & $\mathbf{W V}^{*}$ & $\mathbf{J V}^{*}$ & $\mathrm{JM}^{*}$ & INTER** \\
\hline \multicolumn{9}{|l|}{ Cervical VEMPs: } \\
\hline p13 latency & 0.99 & 0.97 & 0.99 & 0.99 & 0.90 & 0.91 & 0.85 & 0.99 \\
\hline n23 latency & 0.98 & 0.98 & 0.93 & 0.96 & 0.90 & 0.91 & 0.80 & 0.95 \\
\hline p13-n23 amplitude & 0.98 & 0.98 & 0.98 & 0.99 & 0.96 & 0.96 & 0.99 & 0.99 \\
\hline \multicolumn{9}{|l|}{ Ocular VEMPs: } \\
\hline n1 latency & 0.85 & 0.98 & 0.95 & 0.96 & 0.87 & 0.98 & 0.98 & 0.90 \\
\hline p1 latency & 0.97 & 0.99 & 0.98 & 0.99 & 0.72 & 0.99 & 0.98 & 0.87 \\
\hline n2 latency & 0.95 & 0.98 & 0.95 & 0.97 & 0.70 & 0.99 & 0.88 & 0.82 \\
\hline p2 latency & 0.95 & 0.97 & 0.95 & 0.97 & 0.92 & 0.94 & 0.91 & 0.90 \\
\hline n1-p1 amplitude & 0.99 & 0.99 & 0.99 & 0.99 & 0.96 & 0.97 & 0.99 & 0.99 \\
\hline n2-p2 amplitude & 0.99 & 0.97 & 0.98 & 0.99 & 0.99 & 0.99 & 0.99 & 0.99 \\
\hline
\end{tabular}

* Intraobserver reliability ICC values. ${ }^{* *}$ Interobserver reliability ICC values between the three different clinical neurophysiologists.

Table 2.

Intra- and interobserver reliability concerning the forehead tap evoked VEMP test and re-test assessments done by three different experienced clinical neurophysiologists (WV, JV, and JM) and analysed by the intraclass correlation coefficient (ICC, two way random effect model, average measures, and absolute agreement). We refer to the text for further explanation.

\begin{tabular}{|c|c|c|c|c|c|c|c|c|}
\hline & \multicolumn{4}{|c|}{ Forehead tap evoked VEMPs (test) } & \multicolumn{4}{|c|}{$\begin{array}{l}\text { Acoustically evoked VEMPs } \\
\text { (re-test) }\end{array}$} \\
\hline & $\mathbf{W V}^{*}$ & $\mathrm{JV}^{*}$ & $\mathrm{JM}^{*}$ & INTER $^{* *}$ & $\mathbf{W V}^{*}$ & $\mathbf{J V}^{*}$ & $\mathbf{J M}^{*}$ & INTER $^{* *}$ \\
\hline \multicolumn{9}{|l|}{ Cervical VEMPs: } \\
\hline p13 latency & 0.90 & 0.81 & 0.87 & 0.95 & 0.92 & 0.99 & 0.99 & 0.99 \\
\hline n23 latency & 0.98 & 0.98 & 0.80 & 0.98 & 0.99 & 0.99 & 0.96 & 0.94 \\
\hline p13-n23 amplitude & 0.99 & 0.98 & 0.99 & 0.99 & 0.99 & 0.98 & 0.98 & 0.98 \\
\hline \multicolumn{9}{|l|}{ Ocular VEMPs: } \\
\hline n1 latency & 0.99 & 0.83 & 0.73 & 0.80 & 0.86 & 0.77 & 0.75 & 0.90 \\
\hline p1 latency & 0.99 & 0.97 & 0.98 & 0.80 & 0.82 & 0.86 & 0.64 & 0.88 \\
\hline n2 latency & 0.99 & 0.99 & 0.98 & 0.80 & 0.33 & 0.73 & 0.47 & 0.83 \\
\hline p2 latency & 0.96 & 0.99 & 0.76 & 0.76 & 0.47 & 0.89 & 0.49 & 0.70 \\
\hline n1-p1 amplitude & 0.78 & 0.99 & 0.99 & 0.89 & 0.99 & 0.99 & 0.99 & 0.99 \\
\hline n2-p2 amplitude & 0.99 & 0.99 & 0.99 & 0.99 & 0.99 & 0.99 & 0.99 & 0.99 \\
\hline
\end{tabular}

* Intraobserver reliability ICC values. ${ }^{* *}$ Interobserver reliability ICC values between the three different clinical neurophysiologists. 


\section{Table 3.}

Test re-test reliability concerning the acoustically and forehead tap evoked VEMP assessments done by three different experienced clinical neurophysiologists (WV, JV, and JM) and analysed by the intraclass correlation coefficient (ICC, two way random effect model, average measures, and absolute agreement). We refer to the text for further explanation.

\begin{tabular}{lccccc}
\hline & \multicolumn{2}{c}{ First test } & \multicolumn{2}{c}{ Re-test } & \multirow{2}{*}{ ICC $^{* * *}$} \\
\cline { 2 - 4 } & Mean (SD)* & Range** & Mean (SD)* & Range** & \\
\cline { 2 - 4 } cVEMP (acoustic) & & & & \\
p13 latency (ms) & $15.7(2.2)$ & $11.3-20.1$ & $15.9(2.2)$ & $11.5-20.3$ & 0.56 \\
n23 latency (ms) & $25.1(2.8)$ & $19.5-30.7$ & $24.9(2.6)$ & $19.7-30.1$ & 0.23 \\
p13n23 amplitude (mcV) & $55.5(58.2)$ & $<171.9$ & $61.4(78.9)$ & $<219.2$ & 0.87 \\
cVEMP (forehead tap) & & & & \\
p13 latency (ms) & $15.0(3.0)$ & $9.0-21.0$ & $16.5(3.3)$ & $9.9-23.1$ & 0.73 \\
n23 latency (ms) & $25.2(2.7)$ & $19.8-30.6$ & $25.0(3.0)$ & $19.0-21.0$ & 0.74 \\
p13n23 amplitude (mcV) & $180.0(131.8)$ & $<443.6$ & $161.4(75.4)$ & $<312.2$ & 0.76 \\
oVEMP (acoustic) & & & & & \\
n1 latency (ms) & $12.1(2.2)$ & $7.7-16.5$ & $11.9(2.0)$ & $7.9-15.9$ & 0.41 \\
p1 latency (ms) & $17.8(2.3)$ & $13.2-22.4$ & $17.2(2.3)$ & $12.6-21.8$ & 0.053 \\
n2 latency (ms) & $24.5(3.2)$ & $18.1-30.9$ & $22.6(2.8)$ & $17.0-28.2$ & -0.44 \\
p2 latency (ms) & $31.1(4.6)$ & $21.9-40.3$ & $28.2(4.1)$ & $20.0-36.4$ & -0.45 \\
n1p1 amplitude (mcV) & $4.9(6.1)$ & $<17.1$ & $3.6(3.9)$ & $<11.4$ & 0.51 \\
n2p2 amplitude (mcV) & $4.9(4.0)$ & $<12.9$ & $3.5(2.9)$ & $<12.8$ & 0.50 \\
oVEMP (forehead tap) & & & & \\
n1 latency (ms) & $7.4(1.5)$ & $4.4-10.4$ & $7.9(1.9)$ & $4.1-11.7$ & -0.17 \\
p1 latency (ms) & $12.0(1.8)$ & $8.4-15.6$ & $12.0(1.6)$ & $8.8-15.2$ & 0.24 \\
n2 latency (ms) & $17.4(2.4)$ & $12.6-22.2$ & $17.1(1.5)$ & $14.1-20.1$ & 0.37 \\
p2 latency (ms) & $22.9(2.9)$ & $17.1-28.7$ & $22.4(2.1)$ & $18.2-26.6$ & 0.60 \\
n1p1 amplitude (mcV) & $16.4(14.7)$ & $<45.8$ & $18.1(21.3)$ & $<60.7$ & 0.64 \\
n2p2 amplitude (mcV) & $27.3(24.5)$ & $<76.3$ & $44.0(49.7)$ & $<143.4$ & 0.59 \\
\hline
\end{tabular}

${ }^{*}$ Mean (standard deviation). ${ }^{* *}$ Range is defined as the mean $\pm 2 \mathrm{x}$ standard deviations. ${ }^{* *}$ Test re-test reliability ICC

\section{Discussion}

The primary aim of our study was to determine the intra- and interobserver reliability in and between three experienced clinical neurophysiologists (WV, JV, and JM). However, JV was the only clinical neurophysiologist with extensive experience in performing and assessing VEMPs, and the other two experienced clinical neurophysiologists (WV and JM) did not have any clinical experience in relation to the assessment of VEMPs. Therefore, a consensus meeting was planned at the beginning of our study, at which the local guidelines concerning ocular and cervical VEMP assessments were run through, in combination with a general instruction during a period of about forty-five minutes. Our results show that the intra- and interobserver reliability is excellent overall and especially for the most important 
Table 4.

Comparison of the test re-test intraclass correlation coefficient (ICC) concerning the cervical and ocular VEMP parameters (i.e. $\mathrm{p} 13, \mathrm{n} 23, \mathrm{n} 1$, and $\mathrm{p} 1$ latencies; $\mathrm{p} 13 \mathrm{n} 23$ and $\mathrm{n} 1 \mathrm{p} 1$ peak-to-peak amplitudes) of the present study with the existing literature. We refer to the text for further explanation.

\begin{tabular}{lcccccc}
\hline & \multicolumn{3}{c}{ Cervical VEMP } & \multicolumn{3}{c}{ Ocular VEMP } \\
\cline { 2 - 7 } & $\mathbf{p 1 3}^{*}$ & $\mathbf{n 2 3}$ & $\mathbf{p 1 3 n 2 3}^{* *}$ & $\mathbf{n 1}^{*}$ & $\mathbf{p 1}^{*}$ & $\mathbf{n 1}^{*} \mathbf{1}^{* *}$ \\
\hline Forehead tap evoked & & & & & & \\
Present study & 0.73 & 0.74 & $\mathbf{0 . 7 6}$ & -0.17 & 0.24 & 0.51 \\
Nguyen et al. (2010) [8] & 0.57 & 0.63 & 0.54 & -0.033 & 0.44 & $\mathbf{0 . 8 2}$ \\
Acoustically evoked & & & & & & \\
Present study & 0.56 & 0.23 & $\mathbf{0 . 8 7}$ & -0.17 & 0.24 & 0.64 \\
Eleftheriadou et al. (2009) [5] & $0.61 / \mathbf{0 . 7 8}$ & $\mathbf{0 . 8 3 / 0 . 7 6}$ & $\mathbf{0 . 9 2} / \mathbf{0 . 8 9}$ & - & - & - \\
Isaradisaikul et al. (2008) [6] & $0.37 / 0.64$ & $0.65 / 0.70$ & $\mathbf{0 . 8 6 / 0 . 8 1}$ & - & - & - \\
Maes et al. (2009) [7] & $\mathbf{0 . 7 8}$ & $\mathbf{0 . 8 2}$ & $\mathbf{0 . 9 0}$ & - & - & - \\
Nguyen et al. (2010) [8] & 0.33 & 0.71 & 0.68 & 0.17 & 0.055 & $\mathbf{0 . 7 9}$ \\
Qian et al. (2012) [12] & $\mathbf{0 . 8 0}$ & $\mathbf{0 . 9 3}$ & $\mathbf{0 . 9 7}$ & - & - & - \\
Vanspauwen et al. (2009) [17] & 0.63 & $\mathbf{0 . 8 1}$ & $\mathbf{0 . 7 7}$ & - & - & - \\
Versino et al. (2001) [19] & $0.69 / 0.74$ & $\mathbf{0 . 8 9} / \mathbf{0 . 8 6}$ & $\mathbf{0 . 8 3} / \mathbf{0 . 7 6}$ & - & - & - \\
\hline
\end{tabular}

* cervical and ocular VEMP latencies. ${ }^{* *}$ cervical and ocular VEMP peak-to-peak amplitudes. VEMP parameters with ICC values corresponding with an excellent reliability are printed in bold.

VEMP parameters (i.e. ocular VEMPs: n1, p1 latencies, and n1-p1 peak-to-peak amplitude; cervical VEMPs: p13, n23 latencies, and p13-n23 peak-to-peak amplitude). Therefore, we demonstrated that experienced clinical neurophysiologists without clinical experience in relation to VEMP assessment can be trained in a very short time period, and that these assessments show an excellent reliability both between and within the assessors. To our knowledge, we are the first to study this important aspect of clinical VEMP assessment.

The second aim was to study the test re-test reliability for both forehead tap and acoustically elicited cervical and ocular VEMPs. We refer to table 4 for the comparison of our cervical VEMP test re-test reliability results with the existing literature. The raw peak-to-peak p13n23 amplitude seems to be the most reliable cervical VEMP parameter with an excellent test re-test reliability in both forehead tap and acoustically elicited cervical VEMPs, followed by the forehead tap evoked cervical VEMP latencies which consistently show a good reliability. The test re-test reliability of the acoustically evoked cervical VEMP latencies show results that fluctuate between poor and excellent, which are inconsistent and therefore less reliable. The studies have a few methodological differences, which may possibly explain these different results: (a) that in our and in some other studies ${ }^{18,19,21,22}$ patients were tested sitting in an upright position while in other studies ${ }^{15,17}$ patients were tested in a recumbent position, (b) that some studies used unilateral sternocleidomastoid muscle (SCM) activation ${ }^{9,17,18,21}$ (i.e. by rotating 
the head to the contralateral shoulder) while our and other studies ${ }^{15,19,22}$ used bilateral SCM activation (i.e. by flexing the neck), (c) that our and most other studies ${ }^{18,19,21,22}$ used pressure gauges to maintain a chosen target pressure during the VEMP measurements while others ${ }^{15,17}$ did not (however, Nguyen et al. (2010) ${ }^{15}$ corrected the raw peak-to-peak p13n23 amplitude after the measurements by dividing the amplitude by the rectified pre-stimulus EMG activity), and (d) that most studies $15,17,18,21$ used disposable surface electrodes without applying abrasive gel before attachment, possibly resulting in higher impedance levels which may have affected the measured VEMP parameters (and especially the amplitudes due to a higher level of background noise). However, it should be noted that all the acoustically and forehead tap evoked cervical VEMP parameters remained within normal limits despite the individual test re-test variability.

There was only one prior study which measured the test re-test variability in ocular VEMPs, which was performed by Nguyen et al. (2010) ${ }^{15}$. We refer to table 4 for the comparison of our ocular VEMP test re-test reliability results with the existing literature. Nguyen et al. (2010) ${ }^{15}$ concluded that the ocular VEMP test re-test reliability was excellent for $\mathrm{n} 1 \mathrm{p} 1$ peak-to-peak amplitudes and poor for the $\mathrm{n} 1$ and $\mathrm{p} 1$ latencies. We agree that the reliability concerning acoustically and forehead tap elicited ocular VEMP latencies is poor, however we found a fair-to-good reliability concerning the n1p1 amplitudes. There also were a few differences between the studies: (a) Nguyen et al. $(2010)^{15}$ used disposable surface electrodes, as was discussed earlier, (b) electrode placement in the study by Nguyen et al. $(2010)^{15}$ was checked by performing vertical saccades before ocular VEMP testing was performed, and the electrode placement was adjusted if there was an amplitude asymmetry of more than 25 percent resulting in an optimal electrode positioning, and (c) the patients in the study by Nguyen et al. $(2010)^{15}$ were tested in a recumbent position while patients in our study were tested while sitting upright. These differences can possibly explain the differences in the test re-test reliability between our studies. Correction of the ocular VEMP electrode placement, if the vertical saccade amplitude asymmetry is greater than 25 percent in particular, as was done by Nguyen et al. $(2010)^{15}$, could possibly significantly enhance the ocular VEMP n1p1 amplitude test re-test reliability. However, it should again be noted that, in spite of the test re-test variability, all the acoustically and forehead tap evoked ocular VEMP parameters remained within normal limits.

To conclude, the assessment of both cervical and ocular VEMPs shows an excellent intra- and interobserver reliability. The raw $\mathrm{p} 13 \mathrm{n} 23$ peak-to-peak amplitude in cervical VEMPs seems to be the most stable and reliable parameter with an excellent test retest reliability, in which the $\mathrm{p} 13$ and n 23 latencies show an overall good reliability. The raw $n 1 \mathrm{p} 1$ peak-to-peak amplitude in ocular VEMPs shows a good-to-excellent test re-test reliability, but the $\mathrm{n} 1$ and $\mathrm{p} 1$ latencies show an overall poor reliability. 
It should, however, be noted that the results of all subjects of both the test and the re-test remained within normal limits notwithstanding their individual variability. Correction of the ocular VEMP electrode position, when vertical saccade amplitudes show a greater asymmetry than 25 percent, seems to be a particular interesting method for further ocular VEMP standardization. This correction is very likely to enhance the n1p1 ocular VEMP raw peak-to-peak amplitude test re-test reliability and we advise further research concerning this method for standardization. 


\section{References}

1. Curthoys, I. S. A critical review of the neurophysiological evidence underlying clinical vestibular testing using sound, vibration and galvanic stimuli. Clin. Neurophysiol. 121, 132-144 (2010).

2. Papathanasiou, E. S., Murofushi, T., Akin, F. W. \& Colebatch, J. G. International guidelines for the clinical application of cervical vestibular evoked myogenic potentials: an expert consensus report. Clin. Neurophysiol. 125, 658-66 (2014).

3. Rosengren, S. M., Welgampola, M. S. \& Colebatch, J. G. Vestibular evoked myogenic potentials: Past, present and future. Clin. Neurophysiol. 121, 636-651 (2010).

4. Rosengren, S. \& Kingma, H. New perspectives on vestibular evoked myogenic potentials. Curr. Opin. Neurol. 26, 74-80 (2013).

5. Venhovens, J., Meulstee, J. \& Verhagen, W. I. M. Vestibular evoked myogenic potentials (VEMPs) in central neurological disorders. Clin. Neurophysiol. 127, 40-49 (2016).

6. Welgampola, M. S. \& Colebatch, J. G. Characteristics and clinical applications of vestibular-evoked myogenic potentials. Neurology 64, 1682-1688 (2005).

7. Brantberg, K. Vestibular evoked myogenic potentials (VEMPs): Usefulness in clinical neurotology. Seminars in Neurology 29, 541-547 (2009).

8. Curthoys, I. S. A balanced view of the evidence leads to sound conclusions. A reply to J.G. Colebatch 'Sound conclusions?'. Clin. Neurophysiol. 121, 977-8 (2010).

9. Eleftheriadou, A. \& Koudounarakis, E. Vestibular-evoked myogenic potentials eliciting: an overview. Eur. Arch. Otorhinolaryngol. 268, 331-9 (2011).

10. Curthoys, I. S., Manzari, L., Smulders, Y. E. \& Burgess, A. M. A review of the scientific basis and practical application of a new test of utricular function--ocular vestibular-evoked myogenic potentials to bone-conducted vibration. Acta Otorhinolaryngol. Ital. 29, 179-86 (2009).

11. Papathanasiou, E. S. Ocular vestibular evoked myogenic potentials (OVEMPs): saccule or utricle? Clin. Neurophysiol. 123, 216 (2012).

12. Papathanasiou, E. S. \& Papacostas, S. S. Vestibular evoked myogenic potentials: the fuzzy picture of different stimulation types is beginning to come into focus. Clin. Neurophysiol. 124, 1926-7 (2013).

13. Todd, N. The ocular vestibular evoked myogenic potential (OVEMP), ten years old. Clin. Neurophysiol. 125, 439-41 (2014).

14. Walther, L. E., Rogowski, M., Hörmann, K., Schaaf, H. \& Löhler, J. Ocular vestibular evoked myogenic potentials to air conduction (AC oVEMP): useful in clinical practice? Otolaryngol. Pol. 65, 333-8

15. Nguyen, K. D., Welgampola, M. S. \& Carey, J. P. Test-retest reliability and age-related characteristics of the ocular and cervical vestibular evoked myogenic potential tests. Otol. Neurotol. 31, 793-802 (2010).

16. Eleftheriadou, A. et al. Test-retest reliability of $\mathrm{p} 13 \mathrm{n} 23$ and n34p44 components of vestibular evoked myogenic potentials in a large healthy population. J. Otolaryngol. - Head Neck Surg. 38, 462-467 (2009).

17. Isaradisaikul, S. et al. Reliability of vestibular evoked myogenic potentials in healthy subjects. Otol. Neurotol. 29, 542-544 (2008).

18. Maes, L. et al. The vestibular evoked myogenic potential: A test-retest reliability study. Clin. Neurophysiol. 120, 594-600 (2009).

19. Qian, W., Houlden, D., Adamonis, J., Chen, J. M. \& Tao, Z. Z. Novel method for vestibular evoked myogenic potentials: technique and reproducibility. J. Otolaryngol. - Head Neck Surgery. Le J. d'Oto-Rhino-Laryngologie Chir. Cervico-Faciale 41, 303-308 (2012).

20. Vanspauwen, R., Wuyts, F. L. \& Van De Heyning, P. H. Validity of a new feedback method for the VEMP test. Acta Otolaryngol. 126, 796-800 (2006).

21. Vanspauwen, R., Wuyts, F. L. \& Van De Heyning, P. H. Vestibular evoked myogenic potentials: Test-retest reliability and normative values obtained with a feedback method for the sternocleidomastoid muscle contraction. J. Vestib. Res. Equilib. Orientat. 19, 127-135 (2009).

22. Versino, M., Colnaghi, S., Callieco, R. \& Cosi, V. Vestibular evoked myogenic potentials: test-retest reliability. Funct. Neurol. 16, 299-309 (2001).

23. Zapala, D. A. \& Brey, R. H. Clinical experience with the vestibular evoked myogenic potential. J. Am. Acad. Audiol. 15, 198-215 (2004). 


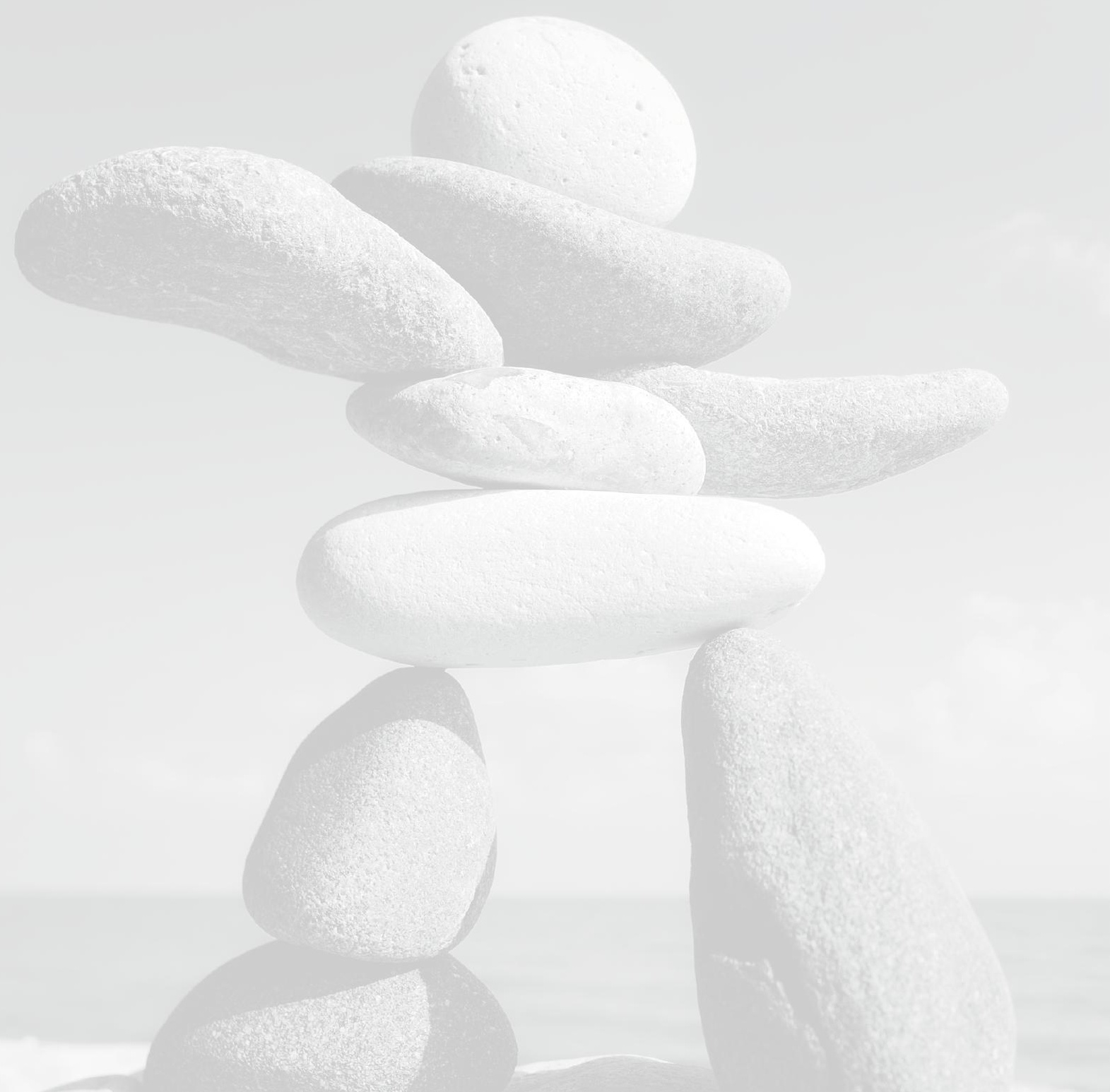




\section{Chapter 4}

Static subjective visual vertical in healthy volunteers:

The effects of different preset angle deviations and test-rest variability

J. Venhovens, J. Meulstee, W.I.M. Verhagen

Neuroophthalmology. 40, 113-119 (2016). 


\section{Abstract}

\section{Objective}

The effects of different preset angle deviations on the static subjective visual vertical (SVV) were assessed in healthy volunteers as the primary aim. The secondary aim was to assess the test re-test variability of the static SVV in healthy volunteers.

\section{Methods}

We recruited twenty-four healthy volunteers for the assessment of the static SVV under different preset angle deviations (i.e. 10, 20, and 30 degrees). In twenty other healthy volunteers the static SVV was tested and re-tested one week later.

\section{Results}

The static SVV did not differ significantly between the different preset angle deviations and between the test re-test measurements, at a group level. However, the combined clockwise deviations and counter-clockwise deviations differed significantly (Wilcoxon test, $\mathrm{P} \leq 0.001$ ). Also, the individual absolute test re-test SVV differences showed clinically relevant variations.

\section{Conclusions}

The static SVV results are influenced by the side of the preset angle, however not by the preset angle deviation (i.e. deviations greater than 10 degrees). The test re-test static SVV outcomes are stable at a group level, however, show clinically relevant differences at the individual level. A robust static SVV protocol was described in this paper. 


\section{Introduction}

Graviception is the perception of a person's orientation relative to the gravitational force, which can be measured by means of the subjective verticals. These subjective verticals can be divided into three tests, which are: a) the subjective visual vertical (SVV), b) the subjective postural vertical (SPV), and c) the subjective haptic vertical $(\mathrm{SHV})^{1}$. These graviceptive pathways integrate the vestibular, the visual, and the proprioceptive inputs, and subsequently estimate the relative position of one's body with respect to the absolute vertical, being the gravitational field of the earth ${ }^{2,7,9}$. The static SVV is an easily applicable test, which aims to detect otolithic imbalance and is a sensitive sign of brainstem dysfunction ${ }^{2}$. The static SVV is tested in complete darkness, in order for visual references to be excluded, and in an upright sitting position, so proprioceptive inputs contribute only minimally. Therefore, the static SVV is almost exclusively determined by the otolithic function and balance, as these are the main receptors in the peripheral vestibular system sensitive to gravitational forces (i.e. linear accelerations), with the semicircular canals being predominantly sensitive to angular accelerations ${ }^{7}$.

Clinical static SVV testing is severely hampered by the lack of a uniform static SVV testing protocol, subsequently resulting in a marked heterogeneity concerning the SVV methodology in the literature. This makes generalization of the SVV findings in the literature very difficult. The main goal of this study is to further explore and propose a robust static SVV protocol, as was already studied in previous reports by Crevits et al. $(2007,2012)^{3,4}$. We defined three aims concerning the SVV methodology and reliability to study in more detail.

The first aim of the present study was to measure the effects of different preset angle deviations on the final static SVV results in healthy volunteers. The second aim was to investigate the test re-test variability in a group of healthy volunteers, as this to our knowledge is unknown in the literature at this moment. We expected that the static SVV findings would not differ significantly at a group level, however, that small variations would very likely exist at an individual level. Therefore, we estimated the size of these variations and the possible clinical relevance. The third aim was to further explore and propose a robust study protocol combining monocular and binocular static SVV measurements by using the method of adjustment. 


\section{Methods}

\section{Study Participants}

From the neurological and clinical neurophysiological hospital staff forty-four healthy volunteers were recruited, none of whom had a relevant medical history (they did not have neurological, otological, and/or ophthalmological diseases or complaints). All had a normal vision or their vision had been corrected to normal with contact lenses or glasses. Clinical neurological and neurotological examinations (i.e. clinical oculomotor examination, testing for a skew-deviation with the alternate cover test, head-impulse test, Dix-Hallpike and supine roll tests) were normal in all healthy volunteers. The study was approved by the regional and local medical ethical committee (CMO Arnhem-Nijmegen, the Netherlands, number 2012/393) and all participants signed an informed consent.

Twenty-four participants (mean age 27 years, range 22-34 years old, 9 men) were included in the study concerning different preset angle deviations and twenty other participants (mean age 24 years, range 21-32 years old, 8 men) were included in the test re-test study.

\section{Tests and procedure}

Before measuring, the system was checked and if necessary adjusted to the absolute vertical with the aid of a plumb line. The subjects were sitting upright and 2 metres away from a viewing screen and we assured that both the (corrected) vision and the visual field of the subjects were sufficient to perform the test. The tests were performed in a totally darkened room to prevent visible landmarks from being seen, and the investigator operated the equipment in an adjacent room. Communication was possible by means of an intercom system. A laser projected straight red line (1.6 metres long and 5 millimetres wide) could be tilted and shown or taken away from a distance by the investigator (VERTITEST-II, Difra Instrumentation, Belgium). The subject was instructed to adjust the laser projected line to the gravitational vertical with a hand-held infrared remote controlled potentiometer (steps of $0.1 \%$ click and with an angle speed of $3.85 \%$ second when the button was pressed continuously) and to wait for 5 seconds at the chosen end position to reconsider and thereafter to vocally confirm this definitive position. After this definitive confirmation the line was taken away by the investigator and tilted to the new starting position before it was projected again to prevent the subject from being influenced. As a convention, deviations counterclockwise from the absolute vertical were indicated by negative values and deviations clockwise from the absolute vertical were indicated by positive values. The deviations were measured with a precision of $0.1^{\circ}$. 
For the first study aim, the static SVV was tested with different preset angle starting deviations. The investigator tilted the laser projected red line at random to a preset angle that was either clockwise or counter-clockwise with a random starting deviation of 10, 20, or 30 degrees. Every starting deviation was tested four times so that a total of twelve measurements were obtained for every testing condition. The measurements were obtained under three different testing conditions in a random order: (i) both eyes viewing, (ii) right eye viewing, and (iii) left eye viewing. When all measurements in all the testing conditions were combined, a total of thirty-six static SVV results were obtained per subject. The lights were turned on for a few minutes after each viewing condition to check the head position and to prevent the SVV drift, resulting from being in the darkness for prolonged periods of time ${ }^{8}$.

For the second study aim, the static SVV was tested at baseline and one week later in the same subject to assess the test re-test variability. The investigator tilted the laser projected red line at random to a preset angle that was either clockwise or counterclockwise with an absolute starting deviation of 20 degrees. The measurements were taken twice so that four measurements were obtained for every testing condition, and measurements were also made for all the three testing conditions, as described above. Thus, a total of twelve static SVV results were obtained per assessment and the test was repeated one week later. Also in this study, the lights were turned on for a few minutes after each viewing condition to check the head position and to prevent the SVV drift, resulting from being in the darkness for prolonged periods of time ${ }^{8}$.

For the third study aim, based on our findings of the observed variance between the methodologies of prior studies, we explored and constructed a robust SVV study protocol, mainly to improve observer variation and to make the test results suitable for comparison among different laboratories.

\section{Statistical analysis}

Because of the non-parametrical distribution of the three dependent groups concerning the different angle preset deviations study (i.e. 10, 20, and 30 degrees), the Friedman test for repeated measures analysis of variance by ranks was applied for comparison. Concerning the two non-parametrically distributed and dependent groups in the test re-test study, the Wilcoxon test was applied for comparison.

Additionally, the test re-test reliability was assessed by the intraclass correlation coefficient (ICC); a two way random effect model with absolute agreement and average measures was used for analysis. The following ICC ranking was adopted: ICC values above 0.75 represent excellent reliability, values between 0.4 and 0.75 represent fair-to-good reliability, and values below 0.4 represent poor reliability. The intraclass correlation coefficient is considered to be the key statistical indicator of relative reliability. The statistical database software SPSS version 23.0 (SPSS inc., USA) was used for statistical analyses. 


\section{Results}

We refer to table 1 for the results concerning the different preset angle deviations study. The Friedman test for repeated measures analysis of variance by ranks showed a P-value $<0.001$ for all binocular and monocular measurements in all different deviations (i.e. 10, 20, and 30 degrees) combined, meaning that some groups differed significantly. We subsequently ran a series of bivariate comparisons (Friedman test) for further analysis between the three groups themselves in different deviations (i.e. 10,20 , and 30 degrees). The binocular and monocular measurements between the different deviations did not differ significantly according to the Friedman test.

The combined clockwise preset angle measurements, which were taken from the three conditions (i.e. binocular and monocular viewing conditions), did not differ significantly either between the different deviations, according to the Friedman test.

The same was true for the combined counter-clockwise preset angle measurements. There was, however, a statistically significant difference between the static SVV results of the combined clockwise preset angle measurements in comparison to the combined counter-clockwise measurements at all deviations separately and combined according to the Wilcoxon test, see table 2 . The same was true for the test re-test measurements in the second part of the study $(\mathrm{P}=0.005$ for the first measurements, and $\mathrm{P}=0.002$ for the re-test measurements, according to the Wilcoxon test).

We refer to table 3 for test re-test study results. At a group level there was no statistically significant difference between the test and re-test measurements, according to the Wilcoxon test.

\section{Table 1.}

Effects of the different preset angle deviations study. The mean deviation for every condition was given in degrees (with the standard deviation). Abbreviations: CW, clockwise starting deviations; CCW, counter-clockwise starting deviations; OS, oculus sinister; OD, oculus dexter; SD, standard deviation.

\begin{tabular}{lcccc}
\hline & $\begin{array}{c}\text { 10 degrees } \\
\text { Mean (SD) [range] }\end{array}$ & $\begin{array}{c}\text { 20 degrees } \\
\text { Mean (SD) [range] }\end{array}$ & $\begin{array}{c}\text { 30 degrees } \\
\text { Mean (SD) [range] }\end{array}$ & $\begin{array}{c}\text { P-Value } \\
\text { (Friedman } \\
\text { test) }\end{array}$ \\
\cline { 2 - 5 } Binocular (CW+CCW) & $-0.4(1.2)[-2.5-2.2]$ & $-0.2(1.3)[-2.3-3.4]$ & $-0.2(1.1)[-3.0-1.4]$ & 0.236 \\
OS (CW+CCW) & $-0.2(1.4)[-3.4-3.1]$ & $-0.2(1.7)[-3.0-4.0]$ & $-0.4(1.6)[-3.1-3.4]$ & 0.243 \\
OD (CW+CCW) & $-0.2(1.4)[-3.1-3.0]$ & $-0.3(1.5)[-2.9-2.6]$ & $-0.2(1.5)[-3.0-2.5]$ & 0.989 \\
CW (all conditions) & $0.1(1.3)[-2.1-3.0]$ & $0.2(1.4)[-2.1-4.3]$ & $0.1(1.5)[-2.7-4.0]$ & 0.409 \\
CCW (all conditions) & $-0.6(1.2)[-2.4-2.3]$ & $-0.6(1.3)[-2.9-2.6]$ & $-0.6(1.0)[-3.0-2.5]$ & 0.620 \\
\hline
\end{tabular}


Also the individual absolute mean differences and standard deviations were calculated for all volunteers, which were defined as: ABSOLUTE $\Delta=$ ABSOLUTE (SVV firs test $\left.-S V V^{\text {re-test }}\right)$. However, at the individual level the test re-test reliability according to the intraclass correlation coefficient was poor overall.

\section{Table 2.}

Data concerning the clockwise starting deviations of all conditions in comparison with the counterclockwise starting deviations of all conditions.

\begin{tabular}{lllll}
\hline & $\begin{array}{l}\text { 10 degrees } \\
\text { Mean }(\text { SD) }\end{array}$ & $\begin{array}{l}\text { 20 degrees } \\
\text { Mean (SD) }\end{array}$ & $\begin{array}{l}\text { 30 degrees } \\
\text { Mean (SD) }\end{array}$ & $\begin{array}{l}\text { All deviations } \\
\text { combined }\end{array}$ \\
\hline CW (all conditions) & $0.1(1.3)$ & $0.2(1.4)$ & $0.1(1.5)$ & $0.1(1.4)$ \\
CCW (all conditions) & $-0.6(1.2)$ & $-0.6(1.3)$ & $-0.6(1.0)$ & $-0.6(1.2)$ \\
P-Value (Wilcoxon test) & $<0.001^{*}$ & $<0.001^{*}$ & $0.001^{*}$ & $<0.001^{*}$ \\
\hline
\end{tabular}

* statistically significant differences with a P-value $<0.05$. Abbreviations: CW, clockwise starting deviations; CCW, counter-clockwise starting deviations; SD, standard deviation.

\section{Table 3.}

Test re-test study. The mean deviation for test was given in degrees (with standard deviation). Also the absolute individual differences between the test and the re-test were given (with the standard deviation) [and range, mean $+/-2 \mathrm{x}$ standard deviations]. Abbreviations: CW, clockwise starting deviations; CCW, counter-clockwise starting deviations; OS, oculus sinister; OD, oculus dexter; SD, standard deviation.

\begin{tabular}{|c|c|c|c|c|c|}
\hline & $\begin{array}{c}\text { First Test } \\
\text { Mean (SD) [range] }\end{array}$ & $\begin{array}{c}\text { Re-Test } \\
\text { Mean (SD) [range] }\end{array}$ & $\begin{array}{c}\text { Absolute } \\
\text { Individual } \\
\text { difference between } \\
\text { tests Mean (SD) } \\
\text { [range] }\end{array}$ & $\begin{array}{l}\text { P-Value } \\
\text { Wilcoxon } \\
\text { test }\end{array}$ & $\begin{array}{l}\text { Intraclass } \\
\text { Correlation } \\
\text { Coefficient }\end{array}$ \\
\hline Binocular $(\mathrm{CW}+\mathrm{CCW})$ & $-0.6(1.1)[-2.7-1.5]$ & $-0.5(1.1)[-2.3-1.6]$ & $1.2(1.0)[0-3.2]$ & 0.705 & -0.095 \\
\hline $\mathrm{OS}(\mathrm{CW}+\mathrm{CCW})$ & $-0.1(1.2)[-1.9-2.0]$ & $-0.3(1.8)[-3.0-2.2]$ & $1.4(1.1)[0-3.6]$ & 0.395 & 0.508 \\
\hline $\mathrm{OD}(\mathrm{CW}+\mathrm{CCW})$ & $-0.4(1.9)[-3.1-3.0]$ & $-0.5(1.7)[-2.9-2.4]$ & 2.0 & 0.896 & -0.240 \\
\hline CW (all conditions) & $-0.8(1.3)[-2.4-2.6]$ & $-0.9(1.2)[-2.7-2.5]$ & $1.2(1.1)[0-3.4]$ & 0.601 & 0.234 \\
\hline CCW (all conditions) & $0(1.4)[-3.5-1.1]$ & $-0.1(1.6)[-2.7-1.5]$ & $1.5(1.2)[0-3.9]$ & 0.507 & 0.251 \\
\hline
\end{tabular}




\section{Discussion}

The first aim of our study was to assess the influence of different preset angle starting deviations (i.e. 10, 20 and 30 degrees) on the final static SVV outcomes. We found a statistically significant difference concerning the side of the preset angle (clockwise or counter-clockwise) in relation to the static SVV outcomes, with the static SVV measurements shifting towards the side of the preset angle. However, this SVV shift did not increase with increasing preset angle deviations, since the influence of the 10 degrees preset angle starting deviation was the same as in the 20 and 30 degrees deviations. This is in concordance with the previously published papers by Pagarkar et al. $(2008)^{6}$ and Baccini et al. $(2014)^{1}$; both noted that the static SVV results were biased in the direction of the preset angle. Baccini et al. $(2014)^{1}$ also tested the static SVV at different preset angle deviations (i.e. 1, 2, 4, 8, and 12 degrees according to the ADJ method) and concluded that the bias was more pronounced at higher deviations, without significant differences between 8 and 12 degrees of deviation. The authors speculated that deviations greater than 8 to 12 degrees do not result in a more pronounced bias of the final static SVV result. However, the methodology of their study in comparison to ours is markedly different, as we tested preset angle deviations with greater deviations and we also included monocular measurements besides the binocular measurements. We confirm the hypothesis of Baccini et al. $(2014)^{1}$ that preset angle starting deviations greater than 12 degrees do not have an additional effect on the static SVV shift.

A few explanations for the shift of the static SVV towards the side of the preset angle have been proposed so far. The first explanation is the entrainment effect proposed and studied by Mezey et al. (2004)5. The entrainment effect states that a rotating environment in the roll plane, but also a rotating line, causes a torsional movement of the eyes in the same direction as the rotation itself. They concluded that this is a kind of optokinesis, which, however, could not be classified as an optokinetic nystagmus since the mean decay time of this effect is about 1 second after cessation of the stimulus and is therefore too slow. This effect is predominantly active in the last 10 degrees of the rotating stimulus in reference to the absolute vertical. Surprisingly, it is not significantly influenced by the presence of a visible non-rotating background. The entrainment effect is present when the laser projected line is both actively or passively rotated. Mezey et al (2004) $)^{5}$ stated that the otoliths have a dampening influence on this entrainment effect; therefore, patients with a disturbed vestibular function have an increased visual reliance and this in combination with the lowered dampening effect results in an increased entrainment effect, secondarily possibly resulting in greater static SVV deviations. This could be the reason why preset angle deviations greater than 8 to 12 degrees do not result in a greater deviation of the static 
SVV towards the preset angle. In our opinion, however, there is one major problem with this explanation; the subjects in our study were instructed to reconsider their final line adjustments for 5 seconds after rotating before approving. In this period the line stood still and was not rotated. The entrainment effect could not play a significant part during this period of reconsideration, since the decay time of this effect is only 1 second. Therefore, the ocular torsion had normalized during the time of reconsideration and the volunteers were, at that moment, still able to change the rotation of the line.

The second explanation is the uncertainty theory ${ }^{1}$, which states that volunteers rotate the line towards the point at which they are uncertain whether the perceived line is already vertical. This uncertainty range is variable between healthy persons and extends from clockwise to counter-clockwise and also encloses the absolute vertical. Most people stop rotating the line when they just entered this uncertainty range and mostly without further rotation, until the moment that they perceive the line as directing towards the opposite side, before re-adjusting the line to their subjective vertical. We support Baccini et al. (2014) $)^{1}$, that the uncertainty theory in combination with the entrainment effect is the most likely explanation for the static SVV bias towards the preset angle. We hypothesize that the subject is at first biased by the entrainment effect, which could possibly increase the subject's uncertainty range during rotation, and is then possibly hesitant to second guess his or her first choice when the entrainment effect subsides. However, we have to state that the hypothesis above is purely speculative and we do not have definitive proof for confirmation.

A tilt in the static SVV could also be induced by tilting the head and/or the body. The E-effect was first described by Muller in 1916; a moderate lateral tilt of the head resulted in a tilt of the static SVV to the contralateral side. A more outspoken lateral tilt of the head and/or the body resulted in a static SVV tilt to the ipsilateral side. This effect is called the A-effect and was first described by Aubert in 1861. ${ }^{9}$ Both effects are thought to be somatosensory in origin. In our study the subjects sat upright in a vertical position in front of the viewing screen and the position was regularly checked during and between the measurements, so we do not believe that either the E-effect or the A-effect can explain our findings.

The second aim of our study was to assess the test re-test variability of the static SVV results. Our study could not demonstrate a significant difference of the static SVV results at a group level between the measurements. However, when the individual absolute differences were calculated, a variation could be seen. For instance, the absolute variation could be up to $3.2^{\circ}$ for binocular viewing, up to $5.2^{\circ}$ for monocular viewing, and up to $3.9^{\circ}$ for all conditions starting with the counter-clockwise preset 
angle. This was supported by the overall poor test re-test intraclass correlation coefficient results (ICC). To our knowledge, we are the first to note this individual static SVV test re-test variation. Tesio et al. $(2011)^{10}$ also calculated the static SVV test re-test reliability showing excellent reliability in young healthy volunteers and fair to good reliability in older volunteers (ICC values were respectively 0.84 and 0.48 ). However, the study of Tesio et al. ${ }^{10}$ has some important differences in comparison to our study, being: a) our study was performed in a totally darkened room to prevent visual references from biasing the static SVV results while Tesio et al. performed their study in a dim-light surrounding, b) the preset angle deviation in our study was greater in comparison with the study of Tesio et al. (respectively 20 and 2.8 degrees) and from the results of Baccini et al. ${ }^{1}$ one can conclude that greater preset angle deviations will result in greater static SVV deviations (with a maximum of 8 degrees), and c) the volunteers in our study used a remote control to manually rotate the laser line, whereas the volunteers in the study by Tesio et al. only had vocal control over the line rotations (rotations were performed manually by a technician) possibly influencing the amount of ocular torsion.

A limitation of our study is that we only studied the test re-test variability in a group of a relatively young age, and not in other groups, especially of a more advanced age. Baccini et al. (2014) ${ }^{1}$ demonstrated that the static SVV measurements were age dependent, and that older persons had more difficulty in judging the absolute vertical, resulting in higher deviations away from the true vertical at higher preset angle deviations (i.e. 8 and 12 degrees). We started our study before the publication of Baccini et al. (2014) ${ }^{1}$, therefore we were not able to look for the older age group. The second limitation is the rather small size of the group of healthy volunteers.

The third and last aim of our study was to explore and propose a robust static SVV study protocol, we refer to the methods section and table 4 for details. The static SVV is a psychophysical test, which can be measured by two methods. The most commonly used method is the adjustment method (ADJ), also known as the method of average error $^{1}$. However, recently Baccini et al. (2014) ${ }^{1}$ extensively tested the static SVV by means of the two-alternative forced choice method (2AFC) in comparison to the ADJ. They concluded that the two testing methods were equally reliable, however, that the 2AFC method was, in their opinion, easier to perform and therefore more practical to use. The main problem concerning static SVV testing, at this moment, is the lack of a uniform testing protocol resulting in a marked heterogeneity in the testing procedures across the different studies. Therefore, the different study results cannot be easily generalized to the everyday clinical practice and furthermore a few methodological issues still need to be addressed. The present study was started before the publication of the article by Baccini et al. $(2014)^{1}$; however, we feel that our study 
Table 4.

Static subjective visual vertical testing protocol.

\section{Static Subjective Visual Vertical (SVV) testing protocol}

Preset angle of 20 degrees (clockwise and counterclockwise)

\begin{tabular}{|c|c|c|}
\hline \multicolumn{3}{|l|}{ Binocular measurements } \\
\hline \multirow[b]{2}{*}{ Clockwise preset angle } & First measurement & Second measurement \\
\hline & $\mathrm{B} 1$ & $\mathrm{~B} 2$ \\
\hline Counter clockwise preset angle & B3 & B4 \\
\hline \multicolumn{3}{|c|}{ Monocular measurements (Oculus Sinister viewing) } \\
\hline \multirow{3}{*}{$\begin{array}{l}\text { Clockwise preset angle } \\
\text { Counter clockwise preset angle }\end{array}$} & First measurement & Second measurement \\
\hline & L1 & $\mathrm{L} 2$ \\
\hline & L3 & L4 \\
\hline \multicolumn{3}{|c|}{ Monocular measurements (Oculus Dexter viewing) } \\
\hline \multirow{3}{*}{$\begin{array}{l}\text { Clockwise preset angle } \\
\text { Counter clockwise preset angle }\end{array}$} & First measurement & Second measurement \\
\hline & $\mathrm{R} 1$ & $\mathrm{R} 2$ \\
\hline & R3 & $\mathrm{R} 4$ \\
\hline \multicolumn{3}{|l|}{ Results } \\
\hline Mean binocular deviation & \multicolumn{2}{|c|}{ Mean (B1, B2, B3, B4) } \\
\hline $\begin{array}{l}\text { Mean monocular oculus } \\
\text { (sinister viewing deviation) }\end{array}$ & \multicolumn{2}{|c|}{ Mean (L1, L2, L3, L4) } \\
\hline $\begin{array}{l}\text { Mean monocular oculus } \\
\text { (dexter viewing deviation) }\end{array}$ & \multicolumn{2}{|c|}{ Mean (R1,R2, R3, R4) } \\
\hline Mean clockwise deviation & \multicolumn{2}{|c|}{ Mean (B1, B2, L1, L2, R1, R2) } \\
\hline Mean counter-clockwise deviation & \multicolumn{2}{|c|}{ Mean (B3, B4, L3, L4, R3, R4) } \\
\hline
\end{tabular}

both supports and complements their findings, as was described earlier. We would like to refer to the methodology section and to prior papers by Crevits et al. (2007 and 2012) $)^{3,4}$ for a detailed overview concerning our proposed SVV protocol. The main advantage of this approach is that both binocular and monocular assessments are systematically made, which may give insight into the nature of the static SVV deviations. For instance, patients with an ocular tilt reaction secondarily resulting from a brainstem infarction are very likely to show an SVV tilt in at least two static SVV conditions, including the binocular viewing condition ${ }^{2}$. Patients with a monocular torsion secondarily resulting from an isolated oblique or vertical ocular motor palsy are very likely to show an abnormal static SVV under ipsilateral monocular viewing conditions, but the other monocular and binocular viewing conditions are 
expected to be normal ${ }^{3,4}$. The proposed study protocol is robust and incorporates 12 static SVV measurements per subject, which can be easily performed clinically in 15 to 20 minutes and which requires only minimal instrumentation.

The reference values for the static SVV testing according to our own normative data by using the protocol discussed above are: a) for binocular measurements $-3.0 \leq \mathrm{x}$ $\leq+3.0$ degrees; b) for monocular measurements $-3.5 \leq \mathrm{x} \leq+3.5$ degrees; $\mathrm{c}$ ) for counterclockwise measurements combined $-5.0 \leq \mathrm{x} \leq+2.0$ degrees; and d) for clockwise measurements combined $-2.0 \leq \mathrm{x} \leq+5.0$ degrees. However, we advise all laboratories to obtain their own reference values in different age groups, as the results of the static SVV are age dependent ${ }^{1}$. 


\section{References}

1. Baccini, M., Paci, M., Del Colletto, M., Ravenni, M. \& Baldassi, S. The assessment of subjective visual vertical: comparison of two psychophysical paradigms and age-related performance. Atten. Percept. Psychophys. 76, 112-22 (2014).

2. Brandt, T. \& Dieterich, M. Vestibular syndromes in the roll plane: topographic diagnosis from brainstem to cortex. Ann. Neurol. 36, 337-47 (1994).

3. Tarnutzer, a a, Bockisch, C., Straumann, D. \& Olasagasti, I. Gravity dependence of subjective visual vertical variability. J. Neurophysiol. 102, 1657-1671 (2009).

4. Yelnik, A. P. et al. Perception of verticality after recent cerebral hemispheric stroke. Stroke 33, 2247-2253 (2002).

5. Crevits, L., Vanacker, L. \& Verraes, A. Patients with migraine correctly estimate the visual verticality. Clin. Neurol. Neurosurg. 114, 313-315 (2012).

6. Crevits, L., Venhovens, J., Vanoutrive, J. \& Debruyne, J. False perception of visual verticality in multiple sclerosis. Eur. J. Neurol. 14, 228-232 (2007).

7. Tarnutzer, A. A., Fernando, D. P., Lasker, A. G. \& Zee, D. S. How stable is perceived direction of gravity over extended periods in darkness? Exp. Brain Res. 222, 427-436 (2012).

8. Pagarkar, W., Bamiou, D.-E., Ridout, D. \& Luxon, L. M. Subjective visual vertical and horizontal: effect of the preset angle. Arch. Otolaryngol. Head. Neck Surg. 134, 394-401 (2008).

9. Mezey, L. E., Curthoys, I. S., Burgess, A. M., Goonetilleke, S. C. \& MacDougall, H. G. Changes in ocular torsion position produced by a single visual line rotating around the line of sight--visual \&quot; entrainment\&quot; of ocular torsion. Vision Res. 44, 397-406 (2004).

10. Tesio, L., Longo, S. \& Rota, V. The subjective visual vertical: validation of a simple test. Int. J. Rehabil. Res. Int. Zeitschrift für Rehabil. Rev. Int. Rech. réadaptation 34, 307-15 (2011). 


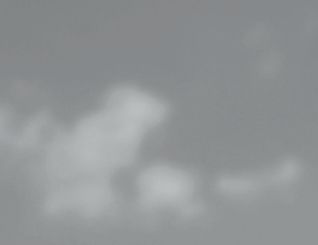

$-\infty$

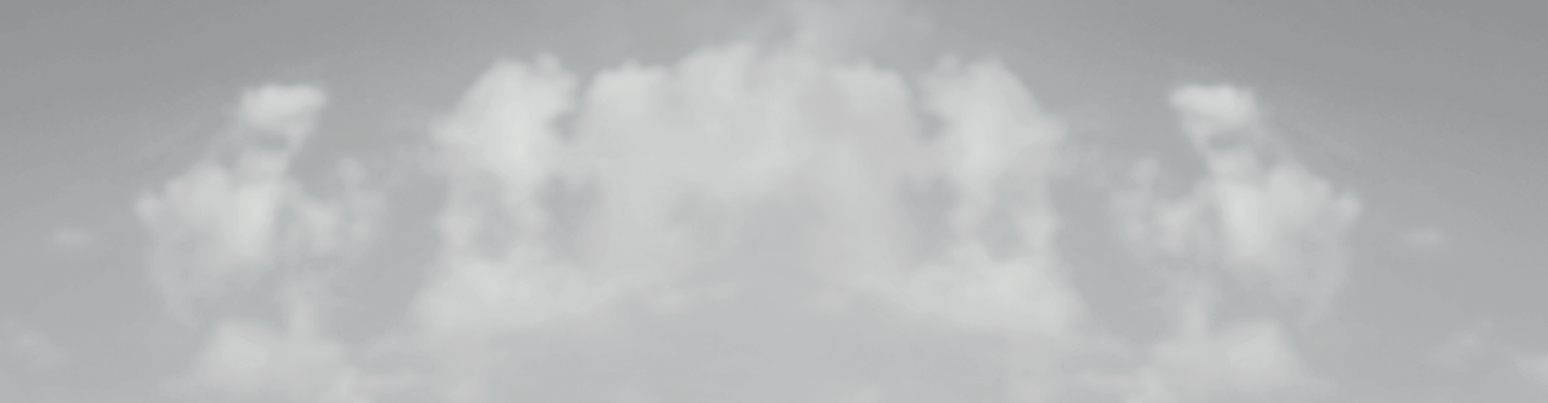




\section{PART TWO}

Neurovestibular analysis and falls in Parkinson's disease and atypical parkinsonism:

A baseline case-control study and prospective follow-up study after one year

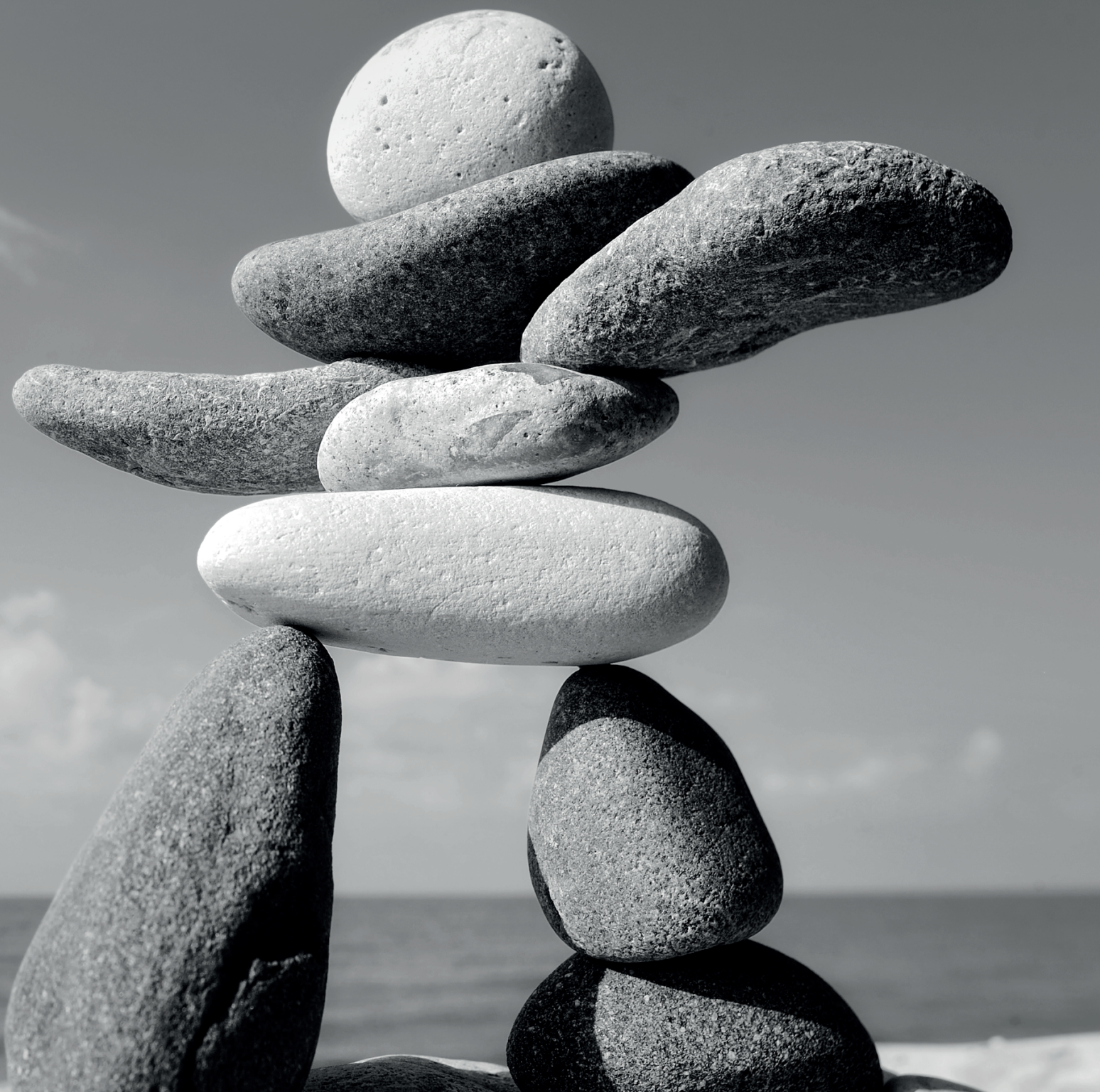




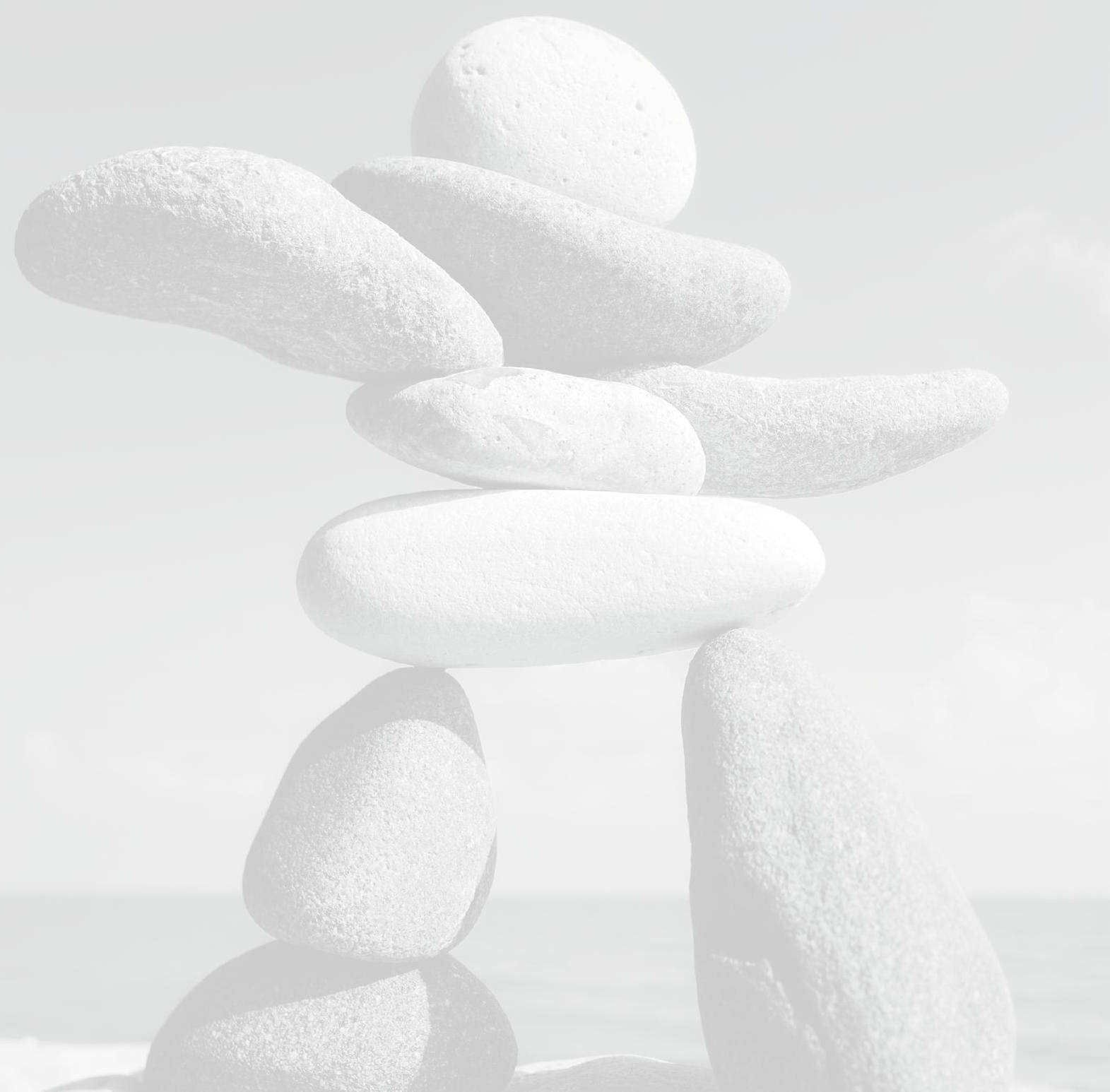




\section{Chapter 5}

Neurovestibular analysis and falls in Parkinson's disease and atypical parkinsonism:

Results from the baseline case-control study

Venhovens, J., Meulstee, J., Bloem, B. R. \& Verhagen, W. I. M.

Eur. J. Neurosci. 43, 1636-46 (2016). 


\section{Abstract}

\section{Objectives}

Our primary aim was to determine the extent of vestibular dysfunction in patients with Parkinson's disease (PD). Our secondary aim was to determine if vestibular dysfunction in PD is a risk factor for falling. The tertiary aim was to determine both the extent of vestibular dysfunction and if this dysfunction is a risk factor for falling in patients with atypical parkinsonism (AP).

\section{Methods}

25 healthy subjects, $30 \mathrm{PD}$ patients, and $14 \mathrm{AP}$ patients were matched for age and gender in a case-control study design. All subjects underwent clinical neurological and neurotological assessments, cervical and ocular vestibular evoked myogenic potentials (VEMPs), brainstem auditory evoked potentials (BAEPs), subjective visual vertical measurements, and videonystagmography with caloric and rotatory chair stimulation.

\section{Results}

$90 \%$ of PD patients (27 of 30) and all 14 AP patients had signs of vestibular dysfunction on laboratory examinations. The evoked potential (VEMPs and BAEPs) test results of PD patients showed significant prolongation of the p13, $\mathrm{n} 1$, interpeak III-V latencies on the symptomatic brainstem side $(0.003 \leq \mathrm{P} \leq$ 0.019) compared to healthy subjects. Also, vestibular testing abnormalities were correlated with an increased risk for falling when fallers among PD and AP patients were compared to the non-fallers $(\mathrm{P} \leq 0.001)$.

\section{Conclusions}

Vestibular dysfunction on vestibular laboratory testing is highly prevalent in both $\mathrm{PD}$ and AP patients compared to healthy subjects, and is associated with an increased risk for falling. 


\section{Introduction}

Postural instability and recurrent falls are a major problem in patients with Parkinson's disease (PD), and even more so for patients presenting with a form of atypical parkinsonism (AP). Approximately $70 \%$ of patients with PD fall each year ${ }^{1}$ and about $50 \%$ have at least two fall incidents annually ${ }^{2-4}$, some of which result in referrals to hospital for treatment of injuries 5 . Falls can induce a fear for renewed falls, resulting in a reduced quality of life because a self-imposed restriction of daily activities can lead to social isolation ${ }^{3,4}$.

A practical consensus-based overview concerning the risk factors and management of falls in PD emphasized the importance of a multifaceted and personalized approach ${ }^{6}$. One of the many contributing factors relates to vestibular dysfunction, but this aspect has thus far received rather limited attention in the literature. Indeed, among the few published studies on this subject, most only contained small case series or case-control studies $^{7-11}$. The vestibular system and especially the vestibular nuclei in the brainstem play a crucial role in integrating multiple sensory information (i.e. visual, proprioceptive, and vestibular sensory input) and to subsequently adjust the outgoing motor response to maintain balance.

The primary aim of this study is to determine the extent of vestibular dysfunction in a group of patients with PD. Four different tests may be useful for this purpose, being: a) the subjective visual vertical (SVV), b) ocular and cervical vestibular evoked myogenic potentials (VEMP), c) the brainstem auditory evoked potentials (BAEP), and d) videonystagmography, rotatory chair tests, and caloric stimulation. The SVV test focusses on the graviceptive pathways (which integrate the vestibular, proprioceptive and visual inputs) and subsequently estimate the relative position of one's body with respect to the absolute vertical (i.e. being the gravitational field of the earth ${ }^{12-15}$. The result of the SVV test is almost exclusively determined by the otolithic function and balance, and also is a sensitive sign for brainstem dysfunction besides the peripheral vestibular otolithic imbalance ${ }^{12,16}$. The combination of both monocular and binocular static SVV measurements may give important insight into the nature of the SVV deviation. For instance patients with a monocular isolated vertical or oblique ocular motor palsy will very likely only show an abnormal static SVV deviation in the ipsilateral viewing condition; whereas patients with an ocular tilt reaction due to a brainstem infarction will very likely show abnormal static SVV deviations in at least two viewing conditions ${ }^{15}$. The cervical and ocular VEMP are easily applicable neurophysiological tests suitable for detailed evaluation of both the peripheral and central vestibular system. Careful interpretation of possible VEMP abnormalities can 
be very helpful in localizing the vestibular abnormalities to the peripheral or central vestibular system ${ }^{17}$. The BAEP does not test the vestibular system, but the peripheral and central auditory systems; however, both the inner ear and the cochlear nerve are an integral part of the peripheral labyrinth and vestibulocochlear nerve. The BAEP is also able to detect and localize brainstem dysfunction. Therefore, the BAEP can be helpful in both localizing and determining the extent of the lesion in combination with the vestibular tests. (Video)nystagmography in combination with rotatory chair and caloric stimulation belong to the oldest and most widely used tests to determine the function of both the peripheral and central vestibular system, and oculomotor systems. However, these caloric and rotatory stimulation tests are mainly limited to the examination of the lateral semicircular canal, superior vestibular nerve and related central vestibular structures. So the combination of all tests described above (i.e. the vestibular testing battery) can give an insight into the function of both the peripheral (i.e. both the semicircular canals, otoliths, and vestibulocochlear nerve) and central vestibular apparatus. Also, abnormalities can be localized more easily to either the peripheral or central vestibular system when the vestibular battery tests are applied integrally.

The secondary aim is to test our hypothesis that vestibular system abnormalities are a risk factor for falling in PD patients. Finally, we want to determine the first two aims in a mixed group of AP patients.

\section{Methodology}

\section{Study Participants}

Sixty-nine volunteers were included in this case-control study; 25 healthy controls (mean age 67, range 42-81 years, 15 men, 10 women), 30 PD patients (mean age 70, range 59-81 years, 26 men, 4 women, all fulfilling the UK Parkinson's Disease Society Brain Bank criteria ${ }^{18}$ ), and $14 \mathrm{AP}$ patients (mean age 68, range 52-81 years, 9 men, 5 women, 6 multiple system atrophy, 3 progressive supranuclear palsy, and 5 vascular parkinsonism). The multiple system atrophy patients with predominant parkinsonism (MSA-P) all fulfilled the diagnostic criteria for probable MSA-P as proposed in the consensus statement by Gilman ${ }^{19}$. Supranuclear palsy patients (PSP) fulfilled the NINDS-SPSP criteria for possible PSP ${ }^{20}$. Vascular parkinsonism patients fulfilled the criteria of the Winikates and Jankovic vascular rating scale ${ }^{21}$. When patients took levodopa, they were tested during the subjective ON-state. 
The healthy controls and the patients did not have a relevant medical history (no relevant neurological, otological, ophthalmological diseases, and/or absence of moderate-to-severe cognitive problems) with the exception of PD or AP (in combination with a related cerebrovascular disorder in the vascular parkinsonism group). The controls were matched for age and gender to the PD and AP patients. All participants completed the study. The study was approved by the regional and local medical ethical committee (CMO Arnhem-Nijmegen, the Netherlands, number 2012/393), was registered in the Dutch trial register (Nederlands Trial Register, NTR3928); all volunteers signed an informed consent.

\section{Clinical neurological tests and questionnaires}

The participants were questioned about their medical history, medication, dizziness, gait and balance problems, prior falls and near falls, motor fluctuations, and freezing of gait. They underwent a detailed neurological and neurotological clinical examination with additional measurements for possible orthostatic hypotension (i.e. blood pressure measurement after lying supine for at least 15 minutes; followed by blood pressure measurements in a standing position after 1,3, and 5 minutes). All PD and AP patients were tested during a regular medication on-state.

All participants completed: a) the 16 items activities-specific balance confidence scale (ABC-16), b) the dizziness handicap inventory (DHI), c) the Edinburgh handedness inventory, d) all subscales of the standardized unified Parkinson's disease rating scale (UPDRS), e) the modified Hoehn and Yahr scale, f) the Schwab and England activities of daily life (ADL) scale, and g) a standardized falls questionnaire ${ }^{22}$.

All participants received a Berg balance scale examination for quantitative balance assessment with additional pull-testing and functional reach testing for the assessment of the degree of postural imbalance. Partial postural imbalance was defined as a normal functional reach test in combination with an abnormal pull-test (i.e. sudden unexpected forceful backward shoulder pull without any specific prior instructions other than to remain standing upright ${ }^{23}$; the patient was able to recover balance in more than two backward steps). Complete postural imbalance was defined as an abnormal pull-test: the patient would have fallen down if the examiner had not been present behind the patient to catch him/her during the fall. Patients that are informed in more detail about this test tend to shift their centre of mass more anterior by leaning forwards in anticipation of the backward shoulder pull, which makes the test less reliable. For this reason patients were not informed in more detail prior to the test. 


\section{Laboratory tests}

\section{Brainstem Auditory Evoked Potentials (BAEP)}

BAEPs were recorded by using $\mathrm{Ag} / \mathrm{AgCl}$-surface electrodes, and abrasive gel was applied before electrode placement to ensure impedance levels below $2 \mathrm{k} \Omega$, with a maximal side-to-side impedance difference of $1 \mathrm{k} \Omega$. Acoustic stimuli consisted of 80 $\mathrm{dBnHL},+/$ - polarity, clicks. Each click lasted $0.1 \mathrm{~ms}$ and was delivered monaurally with a repetition rate of $9.8 \mathrm{~Hz}$ through a calibrated headphone (TDH49P acoustically shielded headphone, Oxford Medical Instruments, England), while $40 \mathrm{dBnHL}$ white noise was delivered to the contralateral ear. The active electrodes were positioned bilaterally pre-auricular, the reference electrode at $\mathrm{Cz}$, and the ground electrode was positioned at Fpz. BAEPs were recorded with a Medelec Synergy T-EP5 EMG/EP machine (Oxford Medical Instruments, Surrey, England); two averages of 1000 stimuli each (bandpass filtered $300-1000 \mathrm{~Hz}$, notch-filtered and without artifact rejection) were compared for reproducibility. The latencies of peaks I, II, III, IV, and V, and the interpeak latencies of I-III, III-V, I-IV were analyzed.

\section{Vestibular Evoked Myogenic Potentials (VEMP)}

The methodology of both cervical and ocular VEMP testing have been described in detail previously ${ }^{24,17}$. All VEMPs were recorded using $\mathrm{Ag} / \mathrm{AgCl}$-surface electrodes; abrasive gel was applied before attaching the electrodes to ensure that the impedance levels were below $2 \mathrm{k} \Omega$, with a maximal side-to-side impedance difference of $1 \mathrm{k} \Omega$. The ground electrode was placed at $\mathrm{Cz}$. For cervical VEMP recording the active electrodes were placed symmetrically over the upper middle part of the sternocleidomastoid muscle bellies with the reference electrode over the sternal manubrium. The patients were asked to sit upright and to push their chins downward against an inflated blood pressure cuff (inflated to a baseline pressure of $60 \mathrm{mmHg}$ ), as proposed by Vanspauwen et al. (2006) ${ }^{25}$. A target pressure of $80 \mathrm{mmHg}$ was chosen with a maximal variance of $2 \mathrm{mmHg}$ above or below this target pressure, which was visually monitored by both the examiner and the subject using the manometer. We interrupted the VEMP measurements every 30 seconds of contraction, with a pause of 30 seconds, to minimize the effects of muscle strain ${ }^{26}$. The peak latencies (in milliseconds, ms) of the p13 and $\mathrm{n} 23$ were measured, as well as the p13-n23 peak-to-peak amplitude (in microvolts, $\mathrm{mcV}$ ). The active electrodes during the ocular VEMP recording were placed symmetrically over the middle part of the lower eyelids, on top of the inferior orbital edges, and the reference electrodes were placed 2 centimeters below them. During the ocular VEMP measurements, subjects were asked to sit upright and to look upwards at a fixed target (upward eye deviation of about 30 degrees). The peak latencies (in milliseconds, ms) of the $\mathrm{n} 1, \mathrm{p} 1, \mathrm{n} 2$, and $\mathrm{p} 2$ were measured, as were the $\mathrm{n} 1-\mathrm{p} 1$ and n2-p2 peak-to-peak amplitudes (in microvolts, $\mathrm{mcV}$ ). 
The cervical and ocular VEMPs were elicited acoustically by means of short tone bursts with an acoustically shielded headphone (TDH49P acoustically shielded headphone, Oxford Medical Instruments, Surrey, England, rise/fall time: $1 \mathrm{~ms}$, plateau time $2 \mathrm{~ms}$, repetition rate $5 \mathrm{~Hz}$, frequency $500 \mathrm{~Hz}, 100 \mathrm{~dB} \mathrm{NHL}$ ). The cervical and ocular VEMPs were recorded with a Medelec Synergy T-EP5 EMG/EP machine (Oxford Medical Instruments, Surrey, England). All evoked responses were amplified (5000x), band-pass filtered $(10-1000 \mathrm{~Hz})$, notch filtered, averaged, and were recorded without artifact rejection. For each trial two hundred acoustic stimuli were averaged. We checked three trials for reproducibility.

\section{Subjective Visual Vertical (SVV)}

The SVV tests were performed in a completely darkened room. We assured that both the (corrected) vision and the visual field of the participants were sufficient to perform the test. The participants were sitting upright 2 metres from the viewing screen. The investigator operated the equipment in an adjacent room (VERTITEST-II, Difra Instrumentation, Eupen, Belgium). The subject was instructed to adjust the laser projected red line (1.6 metres long and 5 millimetres wide) to the gravitational vertical with a hand-held infrared remote controlled potentiometer (steps of $0.1 \%$ click and with an angle speed of $3.85 \%$ second when the button was pressed continuously) and to wait for 5 seconds at the chosen end position to reconsider and thereafter to vocally confirm this definitive position. After this definitive confirmation the line was taken away by the investigator and tilted to the new starting position before it was projected again to prevent the subject from being influenced. As a convention, deviations counter-clockwise from the absolute vertical were indicated by negative values and deviations clockwise from the absolute vertical were indicated by positive values. The deviations were measured with a precision of $0.1^{\circ}$. The investigator tilted the laser projected red line at random to a preset angle that was either clockwise or counterclockwise with a random starting deviation between 20 and 25 degrees. A total of four measurements were obtained for every testing condition. The measurements were obtained under 3 different testing conditions in a random order: (i) both eyes viewing, (ii) right eye viewing, and (iii) left eye viewing. When all measurements in all the testing conditions were combined, a total of 12 static SVV results were obtained per subject. The lights were turned on for a few minutes after each viewing condition to check the head position and to prevent the SVV drift, resulting from being in the darkness for prolonged periods of time ${ }^{27}$.

\section{Videonystagmography, rotatory chair tests, and calorics}

Nystagmography was performed by using a monocular high quality infrared video goggle system (NysStar, Difra instrumentation, Eupen, Belgium). First, the infrared 
video goggle system was calibrated for both the participant's vertical and horizontal eye movements. Then, normal eye movement functions were tested using a standardized testing battery (i.e. slow pursuit eye movements at different velocities both in the horizontal and vertical plane, random saccades with amplitudes of up to -15 to +15 degrees, optokinetic nystagmus at different stimulus velocities of $24 / 42 / 60^{\circ}$ per second in both directions, and eye movements during eccentric gaze both in dark and light conditions). After this, eye movements secondary to rotatory stimulation (MicroTorque, Difra instrumentation, Eupen, Belgium) were studied (i.e. nystagmus during sinusoidal rotatory chair testing in darkness, the effects of visual fixation, and a velocity step test). Caloric stimulation was performed by monaural irrigation of water at $27^{\circ}$ and $44^{\circ}$ Celsius using a standardized testing protocol. Both the degree of vestibular paresis and the directional preponderance were calculated by means of the Jongkees' formulas (mean slow component velocities: RC, right cold; LC, left cold; RW, right warm; LW, left warm caloric stimulation)

- Vestibular paresis formula $=((\mathrm{RC}+\mathrm{RW})-(\mathrm{LC}+\mathrm{LW})) /(\mathrm{RC}+\mathrm{LC}+\mathrm{RW}+\mathrm{LW})($ normal value $\leq 25 \%)$

- Directional preponderance formula $=((\mathrm{RW}+\mathrm{LC})-(\mathrm{LW}+\mathrm{RC})) /(\mathrm{RC}+\mathrm{LC}+\mathrm{RW}+\mathrm{LW})$ (normal value $\leq 30 \%$ )

\section{Statistical analysis}

The statistical database software SPSS version 23.0 (SPSS inc., USA) was used for statistical analyses. The Shapiro-Wilks test was applied to determine normality. Because of the limited sample size of our study and the results of the normality tests we used non-parametrical tests for further statistical analyses. The Kruskal-Wallis one-way analysis by ranks test was applied for comparison of the continuous non-parametrically distributed data of the three independent groups (controls, PD, and AP), the MannWhitney-U test for a group to group comparison, and a significance level of 5 percent was used for all analyses. An ordinal logistic regression analysis was applied for comparison of the categorical variables.

\section{Results}

Individual patient data concerning clinical characteristics, questionnaire outcomes, Berg balance scale results, and outcomes of the laboratory examinations are presented in tables 1,2, and 3. The clinical parameters are summarized in table 4, and the laboratory examinations in table 5 and 6 . The three groups did not differ significantly 
(especially with respect to age) and were thus adequately matched. Orthostatic hypotension, freezing of gait, and postural instability, as well as the number of falls per person each year, the risk for falling and fear of falling were significantly more common in the PD and AP groups in comparison with healthy individuals.

BAEP abnormalities occurred in PD and AP patients only (86\% of the abnormal BAEP responses of both patient groups combined have interpeak III-V latency prolongation, some combined with peak V latency prolongation; 14\% have absent BAEP responses. The mean latencies of the III-V BAEP intervals that are abnormal are $120 \%$ of the upper limit of normal (range 102-134\% ULN; $20 \%>130 \%$ ULN). Abnormal test results of the ocular and cervical VEMP were seen significantly more often in both patient groups. Delayed latencies were about four times more common in abnormal ocular VEMP measurements in comparison with absent responses, and delayed latencies were equally common as absent responses in abnormal cervical VEMP measurements. The mean latencies of the $\mathrm{n} 1$ ocular VEMP that are abnormal are 128\% ULN (range 108-152\% ULN; 56\% > 130\% ULN) and the mean latencies of the p13 cervical VEMP that are abnormal are 134\% ULN (range 101-217\% ULN; $50 \%>130 \%$ ULN). The mean VEMP n1/p13 latencies and n1-p1/p13-n23 amplitudes did not differ significantly between the PD, AP, and the healthy control groups. The number of abnormal SVV results did not differ significantly between the groups. However, all abnormal SVV tests of healthy controls only showed abnormal results in one viewing condition, whereas $75 \%$ of the abnormal SVV tests of the PD group and $100 \%$ of the AP group showed abnormal results in two or more viewing conditions. The videonystagmography results show significantly more abnormal results in the patient groups with respect to saccade and optokinetic testing in comparison with the healthy controls. Saccadic slowing and increased saccade latency (a mean initiation time of more than $250 \mathrm{~ms}$ ) are abnormal results that are equally present in both groups. Abnormal optokinetic test results exclusively showed unilateral decreased responses in two or more optokinetic stimulation velocities. Also, fixation suppression tests during sinusoidal rotatory stimulation in the patient groups were significantly more often abnormal, especially in the AP group. The other videonystagmography, rotatory chair, and caloric tests did not result in significantly more abnormal results between all three of the groups. 
Table 1.

Individual clinical characteristics of patients with Parkinson's disease and atypical Parkinsonism.

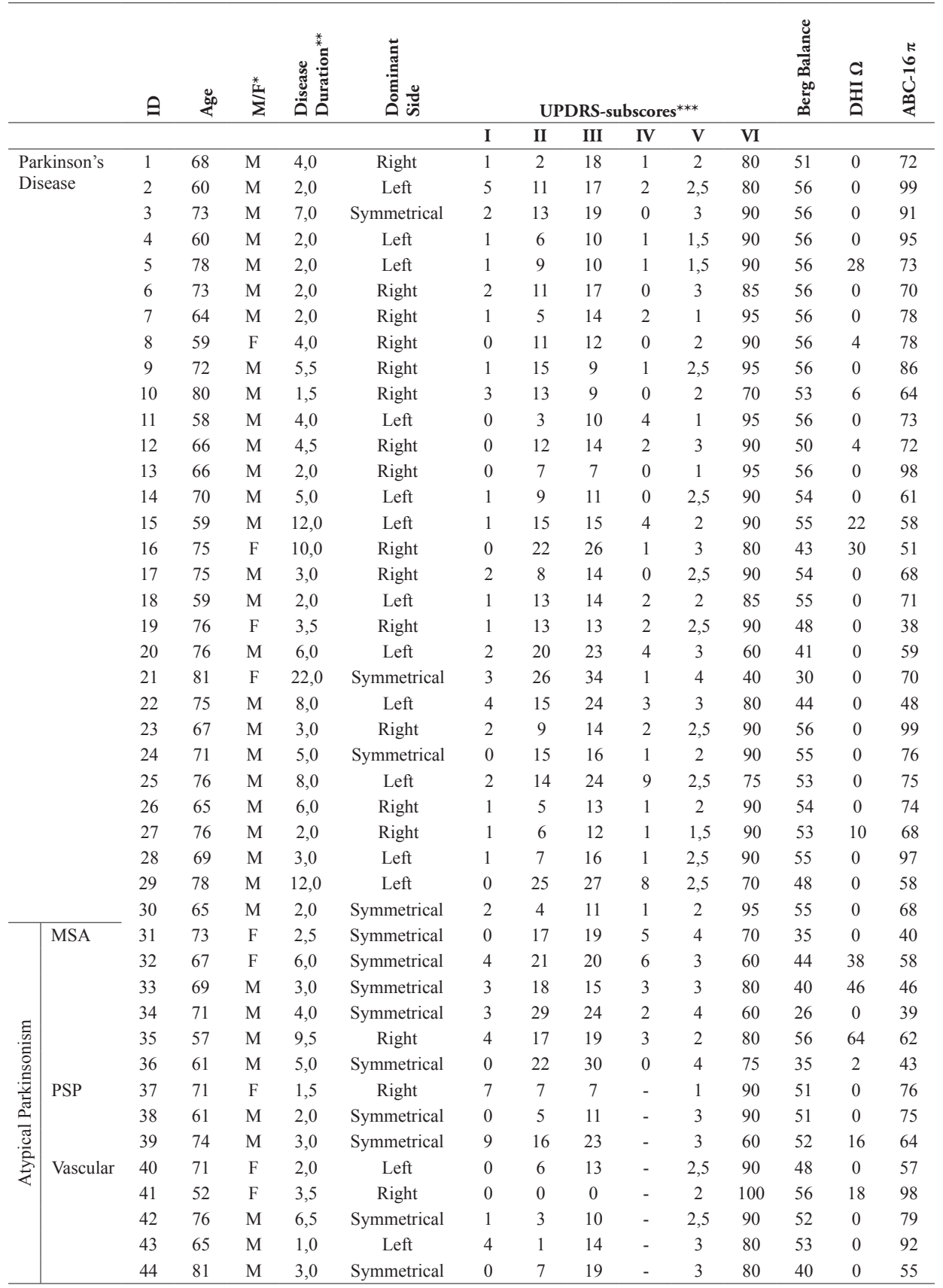

${ }^{*}$ Gender (male or female). ${ }^{* *}$ disease duration (calculated from symptom onset in years). ${ }^{* * *}$ Unified Parkinson's disease rating scale (UPDRS); part I (evaluation of mentation, behavior, and mood); part II (self-evaluation of the activities of daily life, ADL); part III (clinician-scored motor evaluation in the on-state); part IV (Parkinson's disease therapy related complications); part V (Hoehn and Yahr staging of severity of Parkinson's disease); part VI (Schwab and England ADL scale). $\Omega$ DHI (dizziness handicap inventory). $\pi$ ABC-16 (fear of falling questionnaire). 
Table 2.

Individual clinical characteristics and test results of patients with Parkinson's disease and atypical Parkinsonism

\begin{tabular}{|c|c|c|c|c|c|c|c|c|c|c|c|c|c|c|c|c|c|c|}
\hline & & \multirow[t]{2}{*}{ 目 } & \multirow[t]{2}{*}{ 萬 } & \multirow[t]{2}{*}{\begin{tabular}{l}
$*$ \\
*0 \\
\multirow{2}{*}{} \\
0
\end{tabular}} & \multirow[t]{2}{*}{ 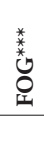 } & \multirow[t]{2}{*}{ 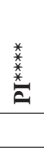 } & \multirow[t]{2}{*}{$\frac{a}{\tilde{g}}$} & \multirow[t]{2}{*}{ 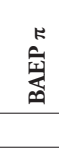 } & \multirow[t]{2}{*}{$\sum_{i=1}^{\infty}$} & \multirow[t]{2}{*}{$\sum_{0}^{\infty}$} & \multirow[t]{2}{*}{$\sum_{\infty}^{\infty}$} & \multicolumn{7}{|c|}{ VNG + calorisation $\varphi$} \\
\hline & & & & & & & & & & & & $\mathrm{C}$ & $S$ & $\mathrm{O}$ & $\mathrm{P}$ & $\mathrm{N}$ & $\mathrm{R}$ & $\mathrm{F}$ \\
\hline \multirow{30}{*}{\multicolumn{2}{|c|}{$\begin{array}{l}\text { Parkinson's } \\
\text { Disease }\end{array}$}} & 1 & - & - & - & - & - & $-1-$ & $-1-$ & $-/ A$ & + & - & - & - & - & - & - & - \\
\hline & & 2 & - & + & + & $\mathrm{C}$ & - & $-1-$ & $-1-$ & $-1-$ & + & $\mathrm{VP}(\mathrm{r})$ & - & - & - & - & - & - \\
\hline & & 3 & $\mathrm{Y}$ & + & - & $\mathrm{C}$ & - & $-/ \mathrm{A}$ & $-1-$ & $\mathrm{A} / \mathrm{A}$ & - & $\mathrm{VP}(\mathrm{l})$ & - & + & + & - & - & - \\
\hline & & 4 & - & - & - & $\mathrm{P}$ & - & $-/-$ & $-1-$ & $-/-$ & - & - & - & - & - & - & - & - \\
\hline & & 5 & - & + & - & $\mathrm{P}$ & - & $-1-$ & $-1-$ & $-1-$ & - & - & - & - & - & - & - & - \\
\hline & & 6 & - & - & - & $\mathrm{C}$ & - & $-/ \mathrm{D}$ & $-1-$ & $-/ \mathrm{A}$ & - & - & + & - & + & - & - & - \\
\hline & & 7 & - & - & - & - & - & $-1-$ & $-/ D$ & $-1-$ & - & - & - & - & - & - & - & - \\
\hline & & 8 & - & - & - & - & - & $-/-$ & $\mathrm{A} /-$ & $-/ \mathrm{D}$ & - & - & - & - & - & - & - & - \\
\hline & & 9 & - & - & - & $\mathrm{P}$ & - & $-1-$ & $-1-$ & $-1-$ & - & - & - & - & + & - & - & - \\
\hline & & 10 & $6 \mathrm{M}$ & - & - & - & - & $-1-$ & $\mathrm{D} /-$ & $\mathrm{D} /-$ & + & - & - & - & + & - & + & - \\
\hline & & 11 & $3 \mathrm{M}$ & - & - & $\mathrm{P}$ & - & $-1-$ & $\mathrm{D} / \mathrm{D}$ & $-/ \mathrm{A}$ & - & - & - & - & - & - & - & - \\
\hline & & 12 & - & - & - & $\mathrm{C}$ & - & $-/ \mathrm{D}$ & $-/-$ & $\mathrm{D} / \mathrm{A}$ & - & VP(l) & + & + & + & - & + & + \\
\hline & & 13 & - & - & - & - & - & $\mathrm{D} /-$ & $-1-$ & $-/ \mathrm{D}$ & - & - & - & - & - & - & - & - \\
\hline & & 14 & - & - & - & $\mathrm{P}$ & - & $-1-$ & $-1-$ & $-1-$ & - & - & - & - & + & - & + & - \\
\hline & & 15 & - & - & - & $\mathrm{P}$ & - & $-1-$ & $-1-$ & $-/ \mathrm{D}$ & - & - & - & - & - & - & - & - \\
\hline & & 16 & $1 \mathrm{M}$ & - & - & $\mathrm{C}$ & + & $-1-$ & $\mathrm{D} /-$ & $-/ A$ & + & - & - & - & + & - & - & - \\
\hline & & 17 & - & - & - & $\mathrm{P}$ & - & $-1-$ & $-1-$ & $-/-$ & + & - & + & + & + & - & - & - \\
\hline & & 18 & - & - & - & - & - & $-1-$ & $-/-$ & $-/-$ & - & - & + & - & + & - & + & - \\
\hline & & 19 & - & - & - & $\mathrm{P}$ & - & D/- & $-1-$ & $-/ \mathrm{D}$ & + & - & + & - & + & - & + & - \\
\hline & & 20 & W & - & + & $\mathrm{C}$ & - & $\mathrm{D} /-$ & $-1-$ & $-/ \mathrm{A}$ & + & - & + & - & + & - & - & - \\
\hline & & 21 & W & - & + & $\mathrm{C}$ & - & $-1-$ & $-1-$ & $\mathrm{D} / \mathrm{A}$ & - & - & - & - & + & - & - & + \\
\hline & & 22 & $1 \mathrm{M}$ & - & + & C & - & $-1-$ & $-/ \mathrm{D}$ & $\mathrm{D} / \mathrm{A}$ & - & - & + & - & + & - & - & - \\
\hline & & 23 & $6 \mathrm{M}$ & + & - & $\mathrm{P}$ & - & $\mathrm{D} /-$ & $-1-$ & $-/ A$ & - & - & - & - & - & - & - & - \\
\hline & & 24 & - & - & - & - & - & $\mathrm{D} / \mathrm{D}$ & $-/ \mathrm{D}$ & $-/ \mathrm{D}$ & + & - & + & - & - & - & - & - \\
\hline & & 25 & $\mathrm{Y}$ & - & + & $\mathrm{P}$ & - & $-1-$ & $\mathrm{D} /-$ & $-/-$ & + & - & + & + & + & - & - & - \\
\hline & & 26 & - & - & - & $\mathrm{P}$ & - & D/- & $-/ \mathrm{D}$ & $-/-$ & - & - & + & - & - & - & - & - \\
\hline & & 27 & - & + & - & - & - & $-1-$ & $-1-$ & $-/-$ & + & - & - & - & + & - & - & - \\
\hline & & 28 & - & - & - & $\mathrm{P}$ & - & $-1-$ & $-1-$ & $-1-$ & + & $\mathrm{VP}(\mathrm{b})$ & + & - & + & - & + & - \\
\hline & & 29 & W & + & + & $\mathrm{P}$ & - & $-1-$ & $\mathrm{A} / \mathrm{D}$ & $-/-$ & - & - & + & + & - & - & - & - \\
\hline & & 30 & $\mathrm{Y}$ & - & - & - & - & $-1-$ & $\mathrm{A} /-$ & $-1-$ & + & - & + & - & + & - & - & - \\
\hline \multirow{14}{*}{ 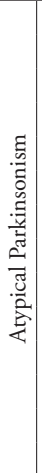 } & \multirow[t]{6}{*}{ MSA } & 31 & $1 \mathrm{M}$ & + & - & C & - & $-1-$ & $-1-$ & $\mathrm{A} / \mathrm{A}$ & - & - & + & + & + & - & - & + \\
\hline & & 32 & $3 \mathrm{M}$ & + & - & C & - & $-1-$ & $\mathrm{D} /-$ & $-/ \mathrm{A}$ & + & - & + & + & + & - & - & + \\
\hline & & 33 & W & + & + & $\mathrm{C}$ & - & $\mathrm{D} / \mathrm{D}$ & $-1-$ & $-/ A$ & - & - & + & + & + & - & + & - \\
\hline & & 34 & W & + & + & $\mathrm{C}$ & - & $-/ \mathrm{A}$ & $\mathrm{A} /-$ & $-1-$ & - & $\mathrm{DP}(\mathrm{l})$ & + & - & + & - & + & - \\
\hline & & 35 & - & + & - & - & + & $-/-$ & $-/ D$ & $-/ A$ & - & - & + & + & + & + & - & + \\
\hline & & 36 & $3 \mathrm{M}$ & + & - & C & - & $-1-$ & $\mathrm{D} / \mathrm{D}$ & $-1-$ & - & - & + & + & + & - & - & + \\
\hline & \multirow[t]{3}{*}{ PSP } & 37 & $6 \mathrm{M}$ & - & - & $\mathrm{P}$ & - & $-1-$ & $\mathrm{D} /-$ & $-1-$ & + & - & + & - & + & - & - & - \\
\hline & & 38 & - & - & - & $\mathrm{C}$ & - & $\mathrm{D} / \mathrm{D}$ & $-1-$ & $-1-$ & - & - & + & - & - & - & - & - \\
\hline & & 39 & - & - & - & $\mathrm{P}$ & - & $-1-$ & $-1-$ & $-/-$ & - & - & + & - & + & - & + & - \\
\hline & \multirow[t]{5}{*}{ Vascular } & 40 & $1 \mathrm{M}$ & + & - & $\mathrm{C}$ & - & $-1-$ & $\mathrm{D} /-$ & $\mathrm{D} /-$ & + & - & - & - & - & - & - & - \\
\hline & & 41 & $6 \mathrm{M}$ & - & - & - & - & $-1-$ & $-1-$ & $-/ \mathrm{A}$ & - & $\mathrm{VP}(\mathrm{r})$ & - & + & + & - & + & + \\
\hline & & 42 & $\mathrm{Y}$ & - & - & $\mathrm{P}$ & - & $\mathrm{D} / \mathrm{D}$ & $-1-$ & $-/-$ & + & - & + & - & + & - & - & - \\
\hline & & 43 & $6 \mathrm{M}$ & - & - & $\mathrm{P}$ & + & $-/-$ & $-1-$ & $-/ \mathrm{A}$ & - & $\mathrm{VP}(\mathrm{r})$ & + & - & + & - & - & - \\
\hline & & 44 & - & - & - & $\mathrm{C}$ & - & $\mathrm{D} /-$ & $\mathrm{D} / \mathrm{D}$ & $\mathrm{D} /-$ & - & - & - & - & - & - & - & - \\
\hline
\end{tabular}

* Number of fall episodes during the last year (Y: once a year; 6M: once every six months; 3M: once every three months; 1M: monthly; W: weekly; D: daily). * Orthostasis (ortho). ${ }^{* * *}$ Freezing of gait (FOG). ${ }^{* * * *}$ Postural imbalance, PI (C: complete imbalance on pull testing without unaided recovery of balance; P: partial imbalance on pull testing with unaided recovery of balance requiring 2 or more backward steps). $\Omega$ Abnormalities during clinical bedside neurovestibular testing. $\pi$ Brainstem Auditory Evoked Potentials (BAEP); Right/Left responses; A: absent response; D: delayed response; -: normal response. $\alpha$ cervical Vestibular Evoked Myogenic Potential (cVEMP). $\beta$ Ocular Vestibular Evoked Myogenic Potential (oVEMP). $\delta$ Subjective Visual Vertical (SVV). $\varphi$ Videonystagmography (VNG) and calorisation results; C(alorisation; VP: vestibular paresis (left/right/bilateral); DP: directional preponderance (left/right/bilateral)); S(accade testing); O(ptokinetics); smooth P(ursuit); spontaneous N(ystagmus) in dark and light conditions; R(otary) chair testing; F(ixation) suppression testing; +: abnormal; -: normal. 
Table 3.

Individual medication characteristics of patients with Parkinson's disease and atypical Parkinsonism.

\begin{tabular}{|c|c|c|c|}
\hline \multirow{3}{*}{\multicolumn{2}{|c|}{$\begin{array}{l} \\
\text { Parkinson's } \\
\text { Disease }\end{array}$}} & \multirow{2}{*}{ ID } & \multirow{2}{*}{$\begin{array}{l}\text { Medication } \\
\text { Levodopa/Benserazide } 300 / 75 \mathrm{mg}+\text { Trihexifenidyl } 1 \mathrm{mg} \text { daily }\end{array}$} \\
\hline & & & \\
\hline & & 2 & Levodopa/Benserazide 300/75mg daily \\
\hline & & 3 & Levodopa/Benserazide 550/137.5mg daily \\
\hline & & 4 & Levodopa/Benserazide 450/112.5mg daily \\
\hline & & 5 & Levodopa/Benserazide 400/100mg daily \\
\hline & & 6 & Levodopa/Benserazide 300/75mg daily \\
\hline & & 7 & Levodopa/Benserazide 800/200mg + Ropinirol 8mg daily \\
\hline & & 8 & Levodopa/Benserazide 800/200mg + Ropinirol 14mg daily \\
\hline & & 9 & Levodopa/Benserazide 400/100mg daily \\
\hline & & 10 & Levodopa/Benserazide 450/112.5mg daily \\
\hline & & 11 & Levodopa/Benserazide $150 / 37.5 \mathrm{mg}$ daily \\
\hline & & 12 & Levodopa/Benserazide 300/75mg daily \\
\hline & & 13 & Levodopa/Benserazide $800 / 200 \mathrm{mg}$ daily \\
\hline & & 14 & Levodopa/Benserazide 400/100mg daily \\
\hline & & 15 & Levodopa/Benserazide 300/75mg + Pramipexol 1.5mg daily \\
\hline & & 16 & Levodopa/Carbidopa/Entacapon 500/125/400mg + Pramipexol 1.5mg daily \\
\hline & & 17 & Levodopa/Benserazide 300/75mg daily \\
\hline & & 18 & Levodopa/Benserazide $450 / 112.5 \mathrm{mg}$ daily \\
\hline & & 19 & Levodopa/Benserazide $600 / 150 \mathrm{mg}$ daily \\
\hline & & 20 & Levodopa/Benserazide 300/75mg daily \\
\hline & & 21 & Levodopa/Benserazide 300/75mg daily \\
\hline & & 22 & Levodopa/Benserazide 900/175mg daily \\
\hline & & 23 & Levodopa/Benserazide 400/100mg daily \\
\hline & & 24 & Levodopa/Benserazide 550/137.5mg daily \\
\hline & & 25 & Levodopa/Benserazide 400/100mg daily \\
\hline & & 26 & Levodopa/Benserazide 600/150mg daily \\
\hline & & 27 & Levodopa/Benserazide 300/75mg daily \\
\hline & & 28 & Levodopa/Benserazide $450 / 112.5 \mathrm{mg}$ daily \\
\hline & & 29 & Levodopa/Benserazide 900/175mg daily \\
\hline & & 30 & Levodopa/Benserazide $150 / 37.5 \mathrm{mg}$ daily \\
\hline & MSA & 31 & Levodopa/Benserazide 300/75mg daily \\
\hline & & 32 & Levodopa/Benserazide $800 / 200 \mathrm{mg}$ + Ropinirol $8 \mathrm{mg}$ daily \\
\hline & & 33 & - \\
\hline & & 34 & Levodopa/Benserazide $150 / 37.5 \mathrm{mg}$ daily \\
\hline$\Xi$ & & 35 & - \\
\hline$\ddot{0}$ & & 36 & - \\
\hline 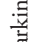 & PSP & 37 & - \\
\hline$\stackrel{\pi}{e}$ & & 38 & - \\
\hline s. & & 39 & - \\
\hline & Vascular & 40 & - \\
\hline & & 41 & - \\
\hline & & 42 & - \\
\hline & & 43 & Levodopa/Benserazide $450 / 112.5 \mathrm{mg}$ daily \\
\hline & & 44 & - \\
\hline
\end{tabular}


The combined results of the vestibular testing battery were significantly more frequently abnormal in the patient groups in comparison to the healthy control group, 93\% in PD and 100\% in AP compared to 60\% in the healthy controls. One patient in the PD group only had abnormal slow eye movement abnormalities during videonystagmography testing, which could also be caused by a lesion outside the vestibular pathways. Therefore, 27 of the PD patients (90\%) and 14 of the AP patients $(100 \%)$ have vestibular abnormalities on laboratory examinations. Of these patients $22 \mathrm{PD}$ patients (79\%) and $8 \mathrm{AP}$ patients (57\%) have no complaints of dizziness or vertigo. The vestibular deficits have a central profile in 21 of the $27 \mathrm{PD}$ patients $(78 \%)$ compared to 13 of the $14 \mathrm{AP}$ patients (93\%). Four PD patients (15\%) and $1 \mathrm{AP}$ patient $(7 \%)$ have a vestibular testing profile that was non localizing. One PD patient (4\%) has a testing profile suggestive of a peripheral vestibular problem.

The BAEP and both cervical and ocular VEMP test results of the symptomatic side in PD patients differed significantly when compared to healthy volunteers. The BAEP I-III and III-V interlatencies differed significantly on the symptomatic side of the PD patients (i.e. $\mathrm{P}=0.012$ and $\mathrm{P}=0.019$, respectively) in comparison to the healthy controls. The other BAEP parameters did not have significantly different results. Also, the cervical VEMP p13 latency and the ocular VEMP n1/p1 latencies had significantly different results on the symptomatic side in comparison to the healthy controls (cervical VEMP p13: P = 0.003; ocular VEMP n1: $\mathrm{P}=0.017$ and p1: $\mathrm{P}=0.009$ ). However, the comparison of BAEP and ocular VEMP results of the asymptomatic side of PD patients to the healthy controls or the side-to-side comparison within PD patients did not yield significantly different results; with exception of the p13 cervical VEMP latency comparison of the symptomatic side versus the asymptomatic side ( $\mathrm{P}$ $=0.020$ ). Also the other cervical and ocular VEMP variables did not differ significantly. Figure 1 shows that neurovestibular test abnormalities are significantly more common in the patients with falling incidents versus the non-falling patients. Eleven of the 30 PD patients fall at least once each year (37\%); 3 patients have orthostasis (27\%), 9 have postural instability (82\%), 5 have freezing of gait (45\%), and 11 have laboratory signs of vestibular dysfunction (100\%). If the number of patients with falling incidents was diminished with the number of falls due to one of the other causes mentioned above, 2 of the 11 PD patients (18\%) only had laboratory signs of vestibular dysfunction. Ten of the $14 \mathrm{AP}$ patients fall at least once each year (71\%); of the falling patients 6 have orthostasis $(60 \%), 2$ have freezing of gait (20\%), 9 have postural imbalance (90\%), and 10 have laboratory signs of vestibular dysfunction (100\%). Only 1 patient $(10 \%)$ has laboratory signs of vestibular dysfunction as the cause for falling, when other causes as mentioned above were corrected. The mean levodopa dosage in falling PD patients was $510 \mathrm{mg} /$ day $(\mathrm{SD} \pm 84 \mathrm{mg}$ ), and in non-falling PD 


\section{Table 4.}

Group characteristics and comparison between the groups concerning the different clinical variables in the Parkinson's disease, atypical Parkinsonism, and healthy control groups.

\begin{tabular}{|c|c|c|c|c|}
\hline & 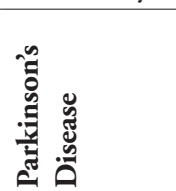 & 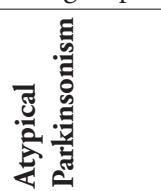 & 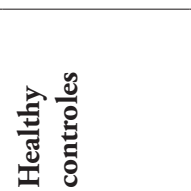 & 苞 \\
\hline Number of patients & 30 & 14 & 25 & 0.054 \\
\hline Gender (male/female) & M: 26 / F: 4 & M: $9 / \mathrm{F}: 5$ & M: $15 / F: 10$ & 0.056 \\
\hline Mean age (years old, SD) & $70(7)$ & $68(8)$ & $67(10)$ & 0.609 \\
\hline Average disease duration (years, SD) & $5,0(4,5)$ & $3,8(2,3)$ & - & 0.373 \\
\hline Orthostasis (N, percentage) & $6(20)$ & $7(50)$ & $0(0)$ & $<0.001$ \\
\hline Freezing of gait (N, percentage) & $6(20)$ & $2(14)$ & $0(0)$ & 0.018 \\
\hline \multicolumn{5}{|l|}{ Postural instability: } \\
\hline - Complete (N, percentage) & $8(27)$ & $8(57)$ & $1(4)$ & $<0.001$ \\
\hline - Partial (N, percentage) & $13(43)$ & $4(29)$ & $1(4)$ & \\
\hline - None (N, percentage) & $9(30)$ & $2(14)$ & $23(92)$ & \\
\hline $\begin{array}{l}\text { Vestibular bedside examination } \\
\text { (normal: N, percentage) }\end{array}$ & $29(97)$ & $12(86)$ & $24(96)$ & 0.387 \\
\hline Handedness (average, SD) & $29,0(3,7)$ & $27,9(5,6)$ & $28,4(5,5)$ & 0.728 \\
\hline ABC-16 fear of falling (average, SD) & $72,9(15,4)$ & $63,1(18,7)$ & $82,3(18,2)$ & 0.006 \\
\hline \multicolumn{5}{|l|}{ UPDRS score (average, SD): } \\
\hline - Subscore I & $1,4(1,2)$ & $2,5(2,9)$ & $0,4(1,2)$ & $<0.001$ \\
\hline - Subscore II & $11,4(6,0)$ & $12,8(8,4)$ & $0,1(0,3)$ & $<0.001$ \\
\hline - Subscore III & $15,8(6,3)$ & $16,0(7,7)$ & $0,1(0,4)$ & $<0.001$ \\
\hline - Subscore IV & $1,8(2,2)$ & $1,9(2,0)$ & $0(0)$ & $<0.001$ \\
\hline - Subscore V & $2,3(0,7)$ & $2,9(0,8)$ & $0(0)$ & $<0.001$ \\
\hline - Subscore VI & $84,7(12,0)$ & $78,9(12,7)$ & $99,2(2,8)$ & $<0.001$ \\
\hline Berg Balance score (average, SD) & $52,2(5,9)$ & $45,6(9,2)$ & $55,4(1,6)$ & $<0.001$ \\
\hline Dizziness Handicap Inventory (average, SD) & $3,5(8,3)$ & $13,1(21,2)$ & $7,4(16,6)$ & 0.239 \\
\hline $\begin{array}{l}\text { Falling patients (at least once in the last year, } \\
\mathrm{N} \text {, percentage) }\end{array}$ & $11(37)$ & $10(71)$ & $3(12)$ & 0.001 \\
\hline \multicolumn{5}{|l|}{ Average number of falls last year: } \\
\hline - All patients in total (average, SD) & $6,4(15,8)$ & $10,2(18,1)$ & $0,3(0,9)$ & 0.001 \\
\hline - Only falling patients (average, SD) & $16,0(22,1)$ & $14,3(20,3)$ & $2,7(1,2)$ & \\
\hline \multicolumn{5}{|l|}{ Fall injury (N, percentage): } \\
\hline - No injury & $1(9)$ & $1(10)$ & $0(0)$ & 0.714 \\
\hline - Minor (e.g. cuts and bruises) & $9(82)$ & $8(80)$ & $2(67)$ & \\
\hline - Intermediate (e.g. simple fractures) & $1(9)$ & $0(0)$ & $1(33)$ & \\
\hline - Severe (e.g. fractures requiring surgery) & $0(0)$ & $1(10)$ & $0(0)$ & \\
\hline \multicolumn{5}{|l|}{ Treatment (N, percentage): } \\
\hline - No treatment necessary & $2(18)$ & $1(10)$ & $0(0)$ & 0.714 \\
\hline - Self-treatment & $6(55)$ & $7(70)$ & $2(67)$ & \\
\hline - Outpatient doctor's treatment & $2(18)$ & $1(10)$ & $1(33)$ & \\
\hline - Hospital admission (no surgery) & $1(9)$ & $0(0)$ & $0(0)$ & \\
\hline - Hospital admission for surgery & $0(0)$ & $1(10)$ & $0(0)$ & \\
\hline
\end{tabular}

* The P-value is calculated by means of an ordinal regression calculation (in the categorical variables) and the Kruskal-Wallis one-way analysis of variance by ranks test (in the continuously distributed, independent, and non-parametrical variables). A significance level of 5 percent (i.e. P-value $\leq 0.05$ ) was adopted for each analysis and significant P-value results are printed in bold. 
patients was $467 \mathrm{mg} /$ day ( $\mathrm{SD} \pm 39 \mathrm{mg}$ ), $\mathrm{P}=0.779$, obviously statistically not different. Nor the disease severity, according to the Hoehn and Yahr classification, of the falling PD patients was statistically significant different from that in the non-falling patients; mean Hoehn and Yahr classification of the falling PD patients was 2.3 (SD \pm 0.6 ), and for the non-falling PD patients was $2.3(\mathrm{SD} \pm 0.9), \mathrm{P}=0.812$.

Table 5.

Group characteristics and comparison between the groups concerning the different test results in the Parkinson's disease, atypical Parkinsonism, and healthy control groups.

\begin{tabular}{|c|c|c|c|c|}
\hline & $\begin{array}{l}\text { Parkinson's } \\
\text { Disease }\end{array}$ & $\begin{array}{l}\text { Atypical } \\
\text { Parkinsonism }\end{array}$ & $\begin{array}{l}\text { Healthy } \\
\text { controles }\end{array}$ & P-value* \\
\hline Number of subjects & 30 & 14 & 25 & 0.054 \\
\hline BAEP abnormal subjects (percentage) $* *$ & $9(30)$ & $5(36)$ & $0(0)$ & 0.072 \\
\hline cVEMP abnormal subjects (percentage) *** & $12(40)$ & $7(50)$ & $2(8)$ & 0.005 \\
\hline oVEMP abnormal subjects (percentage) ${ }^{* * *}$ & $16(53)$ & $8(57)$ & $2(8)$ & $<0.001$ \\
\hline SVV abnormal subjects (percentage) $\alpha$ & $12(40)$ & $4(29)$ & $3(12)$ & 0.057 \\
\hline $\begin{array}{l}\text { VNG + Calorisation abnormal } \\
\text { (percentage): } \varphi \\
\text { - Calorisation (percentage) } \\
\text { - Saccade testing (percentage) } \\
\text { - Optokinetics (percentage) } \\
\text { - Smooth pursuit (percentage) } \\
\text { - Spontaneous nystagmus (percentage) } \\
\text { - Rotary chair testing (percentage) } \\
\text { - Fixation suppression (percentage) }\end{array}$ & $\begin{array}{l}4(13) \\
13(43) \\
5(17) \\
17(57) \\
0(0) \\
6(20) \\
2(7)\end{array}$ & $\begin{array}{l}3(21) \\
11(79) \\
6(43) \\
11(79) \\
1(7) \\
4(29) \\
5(36)\end{array}$ & $\begin{array}{l}2(8) \\
2(8) \\
2(8) \\
10(40) \\
0(0) \\
5(20) \\
0(0)\end{array}$ & $\begin{array}{l}0.627 \\
<0.001 \\
0.035 \\
0.058 \\
0.197 \\
0.795 \\
0.002\end{array}$ \\
\hline $\begin{array}{l}\text { Total neurovestibular testing battery: } \\
\text { oVEMP + cVEMP + VNG + calorisation + } \\
\text { SVV, } \geq 1 \text { abnormal test (percentage) }\end{array}$ & $28(93)$ & $14(100)$ & $17(60)$ & 0.004 \\
\hline $\begin{array}{l}\text { VEMP-testing only (cVEMP+oVEMP): } \\
\geq 1 \text { abnormal test (percentage) }\end{array}$ & $21(70)$ & $11(79)$ & $4(16)$ & $<0.001$ \\
\hline $\begin{array}{l}\text { VEMP-testing (cVEMP+oVEMP) }+ \text { SVV: } \\
\geq 1 \text { abnormal test (percentage) }\end{array}$ & $25(83)$ & $12(86)$ & $6(24)$ & $<0.001$ \\
\hline
\end{tabular}

* The P-value is calculated by means of an ordinal regression calculation (in the categorical variables) and the Kruskal-Wallis one-way analysis of variance by ranks test (in the continuously distributed, independent, and non-parametrical variables). A significance level of 5 percent (i.e. P-value $\leq 0.05$ ) was adopted for each analysis and significant P-value results are printed in bold. ** BAEP: Brainstem auditory Evoked Potentials. ${ }^{* * *}$ cVEMP: cervical Vestibular Evoked Myogenic Potentials, oVEMP: ocular Vestibular Evoked Myogenic Potentials. a SVV: Subjective Visual Vertical. $\varphi$ VNG: videonystagmography. 


\section{Table 6.}

Group characteristics, mean group results, and comparison between the groups concerning the different test results in the Parkinson's disease, atypical Parkinsonism, and healthy control groups.

\begin{tabular}{lllll}
\hline & $\begin{array}{l}\text { Parkinson's } \\
\text { Disease }\end{array}$ & $\begin{array}{l}\text { Atypical } \\
\text { Parkinsonism }\end{array}$ & $\begin{array}{l}\text { Healthy } \\
\text { controles }\end{array}$ & P-value* \\
\hline Number of subjects & 30 & 14 & 25 & 0.054 \\
BAEP** results: mean (SD) & & & & \\
- BAEP 1th peak latency (ms) & $1.54(0.25)$ & $1.44(0.26)$ & $1.54(0.17)$ & 0.867 \\
- BAEP 2th peak latency (ms) & $2.43(0.31)$ & $2.35(0.39)$ & $2.50(0.27)$ & 0.760 \\
- BAEP 3th peak latency (ms) & $3.56(0.39)$ & $3.61(0.41)$ & $3.70(0.25)$ & 0.335 \\
- BAEP 4th peak latency (ms) & $4.71(0.39)$ & $4.77(0.34)$ & $4.78(0.28)$ & 0.557 \\
- BAEP 5th peak latency (ms) & $5.69(0.31)$ & $5.74(0.42)$ & $5.61(0.25)$ & 0.361 \\
- BAEP interpeak I-III latency (ms) & $2.03(0.26)$ & $2.18(0.37)$ & $2.15(0.22)$ & 0.145 \\
- BAEP interpeak III-V latency (ms) & $2.13(0.44)$ & $2.13(0.36)$ & $1.90(0.32)$ & 0.068 \\
- BAEP interpeak I-V latency (ms) & $4.16(0.36)$ & $4.31(0.48)$ & $4.07(0.26)$ & 0.412 \\
Ocular VEMP***: mean (SD) & & & & \\
- n1 peak latency (ms) & $13.7(4.2)$ & $11.8(3.5)$ & $11.5(2.6)$ & 0.067 \\
- p1 peak latency (ms) & $20.8(5.3)$ & $19.4(4.1)$ & $17.5(4.0)$ & 0.021 \\
- n1p1 peak-to-peak amplitude (mcV) & $7.1(11.0)$ & $4.4(2.6)$ & $3.6(3.5)$ & 0.057 \\
Cervical VEMP***: mean (SD) & & & & \\
- p13 peak latency (ms) & $17.0(3.6)$ & $17.0(2.8)$ & $15.1(2.1)$ & 0.143 \\
- n23 peak latency (ms) & $25.1(4.2)$ & $26.0(5.0)$ & $23.3(3.0)$ & 0.318 \\
- p13n23 peak-to-peak amplitude (mcV) & $39.8(45.1)$ & $47.7(66.4)$ & $44.5(49.0)$ & 0.342 \\
SVV $\alpha$ : mean (SD) & & & & \\
- Binocular deviation (degrees) & $0.6(2.4)$ & $-0.3(3.5)$ & $-0.3(1.5)$ & 0.552 \\
- Monocular deviation OS (degrees) & $-0.3(3.0)$ & $0.6(5.4)$ & $-0.9(1.8)$ & 0.234 \\
- Monocular deviation OD (degrees) & $0.4(2.7)$ & $0.1(4.6)$ & $0.3(1.8)$ & 0.918 \\
- Counter-clockwise deviation (degrees) & $-1.0(2.8)$ & $-2.2(4.5)$ & $-1.5(1.6)$ & 0.310 \\
- Clockwise deviation (degrees) & $1.4(3.1)$ & $2.5(5.2)$ & $1.6(1.7)$ & 0.438 \\
\hline
\end{tabular}

* The P-value is calculated by means of the Kruskal-Wallis one-way analysis of variance by ranks test (in the continuously distributed, independent, and non-parametrical variables). A significance level of 5 percent (i.e. $P$-value $\leq 0.05$ ) was adopted for each analysis and significant $P$-value results are printed in bold. ${ }^{* *}$ BAEP: Brainstem auditory Evoked Potentials. ${ }^{* * *}$ VEMP: Vestibular Evoked Myogenic Potentials. a SVV: Subjective Visual Vertical. 


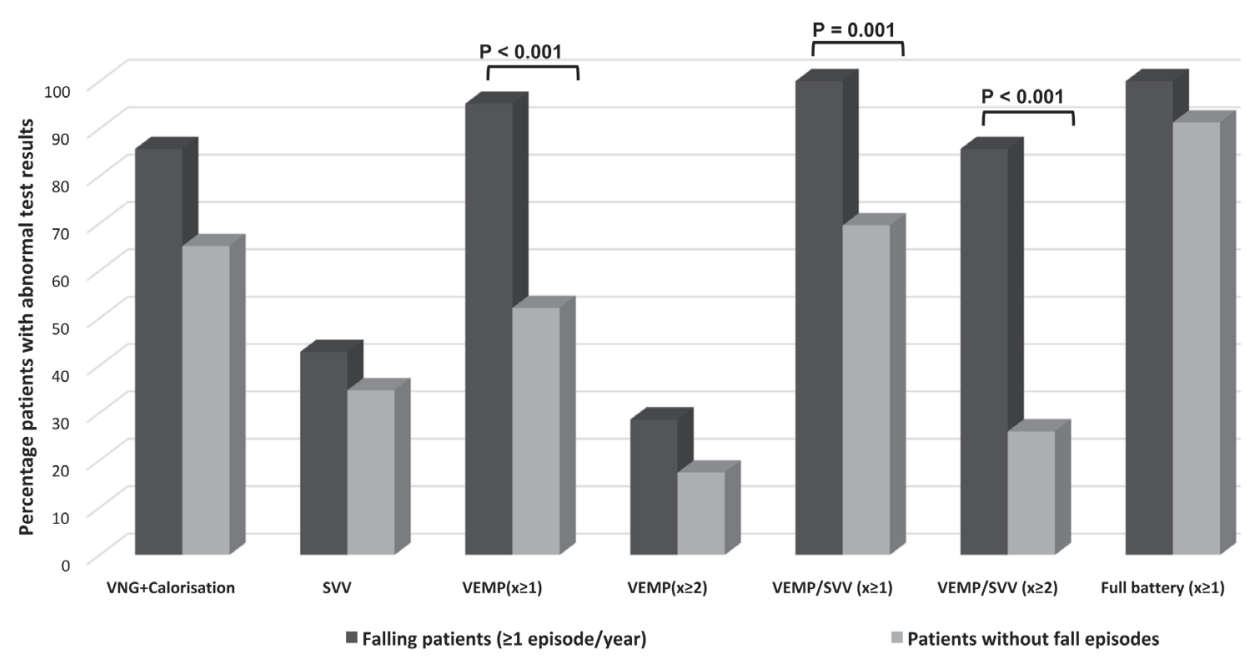

Figure 1.

Sensitivity of the different neurovestibular tests in Parkinson's disease and atypical Parkinsonism patients with and without fall episodes over the last year.

* VNG: videonystagmography, SVV: subjective visual vertical, VEMP: vestibular evoked myogenic potentials, Full battery: full neurovestibular test battery (VNG+calorisation, SVV, and VEMP testing), $\mathrm{x} \geq 1$ : at least one abnormal test result, $\mathrm{x} \geq 2$ : at least two abnormal test results. ${ }^{* *} \mathrm{P}$-values were calculated by means of ordinal regression analysis, only significantly different results (P-value $<0.05$ ) were shown.

\section{Discussion}

In our study abnormal central vestibular function test results are very common, but mostly asymptomatic in both $\mathrm{PD}$ and $\mathrm{AP}$ patients. $\mathrm{PD}$ or AP patients with falling incidents also have significantly more abnormal vestibular test results compared to the non-falling patients. Apart from well-established causes of falls (freezing of gait, orthostasis, cognitive problems, and postural instability), approximately 10 to $18 \%$ of the patients have vestibular dysfunction as the only identifiable cause for falling. We acknowledge that we may have missed additional contributions of e.g. mild orthostasis, which can be variably present during clinical examination, and which can be missed during routine testing ${ }^{28}$. We were not able to exclude all possible and previously reported risk factors for falling in $\mathrm{PD}^{6}$, but we excluded the most relevant causes. Therefore, the present study is mostly exploratory and hypothesis generating, however it holds in our opinion sufficient evidence that vestibular system abnormalities are associated with falling in $\mathrm{PD}$ and $\mathrm{AP}$ patients. To our knowledge this finding has not been reported before; especially the recently published consensus-based overview concerning the risk factors and management of falls in PD did not specifically mention vestibular dysfunction as a contributing cause ${ }^{6}$. 
Pollak ${ }^{7}$ demonstrated vestibular involvement in 54 patients with PD; $37 \%$ had unilaterally absent cervical VEMPs elicited with air-conducted stimuli, and 7\% had bilaterally absent responses; the mean latencies were not significantly different between groups. However, the patients and the control group were not adequately matched for age (PD group: $66 \pm 10$ years, control group: $46 \pm 15$ years, $P<0.001$ ) and the level of sternocleidomastoid muscle contraction was neither standardized, nor corrected for afterwards. Therefore, possible cervical VEMP amplitude differences between the two groups would not have been reliable a priori ${ }^{29}$. Liao ${ }^{8}$ found abnormal air-conducted cervical VEMP p13-n23 amplitudes in 10 patients with PSP and concluded that the reduced vestibulospinal reflex could possibly contribute to the elevated fall risk in PSP patients. The patients and the control group were correctly matched for age, but the cervical VEMP amplitudes were not corrected for the force of sternocleidomastoid muscle correction nor were they standardized, and thus they were not optimally reliable as suggested by the authors. We could not find reduced cervical VEMP amplitudes in the PSP group in our study after age-matching and standardization for the applied force of muscle contraction. Recent studies by De Natale ${ }^{30,31}$ in groups of 33 and 24 PD patients in total showed significantly more abnormal individual masseter, ocular, and cervical VEMPs in comparison with an age matched control group $(\mathrm{P}<0.001)$. At the group level the amplitudes of especially the masseter and ocular VEMPs were significantly smaller in comparison to the healthy control subjects, and the individual results showed a significant correlation with postural instability and REM sleep behavior disorder screening scores. The groups were adequately matched for age and the cervical VEMP amplitudes were corrected for the force of sternocleidomastoid muscle contraction. Therefore, the amplitude differences at the group level in their studies are reliable. These amplitude differences at the group level were not significantly different between the groups in our study, however we agree with the high level of individual VEMP abnormalities in the PD group in comparison to the control group. Pötter-Nerger ${ }^{9}$ concluded that cervical VEMP latencies and amplitudes did not differ significantly between PD patients and healthy controls at a group level. However, ocular VEMP latencies, especially in boneconducted stimuli at the symptomatic side were significantly prolonged, amplitudes on the other hand did not differ significantly. They adequately matched for age to the control group; the level of sternocleidomastoid muscle contraction was standardized. They concluded that the abnormal ocular VEMP latencies were probably caused by upper brainstem involvement, with the lower brainstem being relatively spared (hence the normal cervical VEMPs). Our study confirms the findings of Pötter-Nerger ${ }^{9}$ that ocular VEMP latencies are significantly prolonged at the symptomatic side in comparison to healthy controls. In addition, we found a significantly different result concerning cervical VEMP latencies at the symptomatic side. This difference 
concerning the cervical VEMP latencies can possibly be explained by differences in population size (30 PD patients versus 13 in the study by Pötter-Nerger ${ }^{9}$ ), since smaller differences between groups are easier to be detected in a larger study population. Therefore, we disagree with the conclusion of the previous authors that especially otolith-ocular projections on the symptomatic side in the upper brainstem of $\mathrm{PD}$ patients are more vulnerable to neurodegeneration in comparison to the lower brainstem structures; the otolith-cervical projections through the medial vestibulospinal tract on the symptomatic side are functionally involved (this is the same side as the patient's rigidity, resting tremors, and bradykinesia; as the vestibulospinal tract fibers descent from the vestibular nuclei at the ipsilateral side). Not only the otolith-ocular projections of the symptomatic side of the upper brainstem are especially vulnerable to neurodegeneration, but also the other brainstem projections such as the auditory brainstem pathways seem to be affected, which is illustrated especially by the prolonged BAEP III-V interlatencies. In the peripheral nervous system, considerable prolonged latencies are associated with demyelination. The same principle holds true for prolonged latencies of BAEP components in multiple sclerosis and other demyelinating disorders of the central nervous system. Whether the substantial delay encountered in components of the cervical and ocular VEMP, in both PD and AP patients, is caused by demyelination is rather speculative, although still a subject for further research, possibly with secondary axonal degeneration in the more advanced stages ${ }^{29}$. Primary axonal neurodegeneration is probably less likely to be an explanation for the prolonged latencies without relevant amplitude reduction, which is found in our group of PD and AP patients.

Our study has limitations. The size of the patient groups is rather small due to strict in-/exclusion criteria. The AP group was particularly small and also heterogeneous, so these results must be interpreted cautiously and as hypothesis-generating for further research. Also, the lengthy and cumbersome nature of neurovestibular testing is demanding for elderly patients, and especially for AP patients. Some symptoms (such as mild orthostasis) are fluctuating phenomena; so we are unable to fully exclude all other possible risk factors as a cause for falling. Finally, patients were tested during a subjective on state, so we cannot exclude that dopaminergic medication affected some of our findings. Recently, Pötter-Nerger $e t$ al. performed cervical and ocular VEMP studies in PD patients during both the subjective ON- and OFF-state9. Both the latencies and amplitudes of the cervical and ocular VEMPs did not show effects of dopaminergic modulation. However, in an earlier study Pötter-Nerger et al. demonstrated that PD patients have significantly lower cervical VEMP amplitudes in comparison with healthy controls both during the subjective ON- and OFF-state ${ }^{32}$. The amplitudes of the PD patients, however, were significantly higher during the 
subjective $\mathrm{ON}$-state in comparison to the OFF-state indicating that levodopa partially restores the decreased vestibular nuclear excitability ${ }^{32}$. Our patients were tested during the subjective $\mathrm{ON}$-state. Based upon the literature referenced above it seems likely that levodopa did not have any influence or would possibly partially decrease vestibular testing abnormalities. Therefore, our study may even show some underestimation of vestibular abnormalities.

To conclude, we found a high prevalence of abnormal vestibular test results in both $\mathrm{PD}$ and AP patients; specifically in the symptomatic side of the brainstem in PD patients. The quantitative extent of the electrophysiological abnormalities may suggest a predominant demyelinating component in the neurodegenerative process. The vestibular system abnormalities were associated with falling in both groups. 


\section{References}

1. Bloem, B. R., Steijns, J. A. G. \& Smits-Engelsman, B. C. An update on falls. Curr. Opin. Neurol. 16, 15-26 (2003).

2. Wood, B. H., Bilclough, J. A., Bowron, A. \& Walker, R. W. Incidence and prediction of falls in Parkinson's disease: a prospective multidisciplinary study. J. Neurol. Neurosurg. Psychiatry 72, $721-$ 725 (2002).

3. Grimbergen, Y. A. M., Munneke, M. \& Bloem, B. R. Falls in Parkinson's disease. Curr. Opin. Neurol. 17, 405-415 (2004).

4. Bloem, B. \& Bhatia, K. in Clinical disorders of balance, posture and gait. (ed. Bronstein AM, Brandt T, Nutt JG, W. M.) 173-206 (Arnold: London, 2004).

5. Pressley, J. C. et al. The impact of comorbid disease and injuries on resource use and expenditures in parkinsonism. Neurology 60, 87-93 (2003).

6. van der Marck, M. A. et al. Consensus-based clinical practice recommendations for the examination and management of falls in patients with Parkinson's disease. Parkinsonism and Related Disorders 20, 360-369 (2014).

7. Pollak, L., Prohorov, T., Kushnir, M. \& Rabey, M. Vestibulocervical reflexes in idiopathic Parkinson disease. Neurophysiol. Clin. 39, 235-240 (2009).

8. Liao, K. et al. Why do patients with PSP fall? Evidence for abnormal otolith responses. Neurology 70, 802-809 (2008).

9. Pötter-Nerger, M., Govender, S., Deuschl, G., Volkmann, J. \& Colebatch, J. G. Selective changes of ocular vestibular myogenic potentials in Parkinson's disease. Mov. Disord. 30, 584-9 (2015).

10. Wang, S. R. \& Young, Y. H. Multiple system atrophy manifested as dizziness and imbalance: A report of two cases. Eur. Arch. Oto-Rhino-Laryngology 260, 404-407 (2003).

11. Bassetto, J. M., Zeigelboim, B. S., Jurkiewicz, A. L. \& Klagenberg, K. F. Neurotological findings in patients with Parkinson's disease. Braz J Otorhinolaryngol 74, 350-355 (2008).

12. Brandt, T. \& Dieterich, M. Vestibular syndromes in the roll plane: topographic diagnosis from brainstem to cortex. Ann. Neurol. 36, 337-47 (1994).

13. Tarnutzer, a a, Bockisch, C., Straumann, D. \& Olasagasti, I. Gravity dependence of subjective visual vertical variability. J. Neurophysiol. 102, 1657-1671 (2009).

14. Yelnik, A. P. et al. Perception of verticality after recent cerebral hemispheric stroke. Stroke 33, 2247-2253 (2002).

15. Venhovens, J., Meulstee, J. \& Verhagen, W. Static Subjective Visual Vertical (SVV) in healthy volunteers: the effects of different preset angle deviations and test re-test variability. Neuroophthalmology. 40, 113-119 (2016).

16. Crevits, L., Venhovens, J., Vanoutrive, J. \& Debruyne, J. False perception of visual verticality in multiple sclerosis. Eur. J. Neurol. 14, 228-232 (2007).

17. Venhovens, J., Meulstee, J. \& Verhagen, W. I. M. Vestibular evoked myogenic potentials (VEMPs) in central neurological disorders. Clin. Neurophysiol. 127, 40-49 (2016).

18. Jankovic, J. Parkinson's disease: clinical features and diagnosis. J. Neurol. Neurosurg. Psychiatry 79, 368-76 (2007).

19. Gilman, S. et al. Second consensus statement on the diagnosis of multiple system atrophy. Neurology 71, 670-676 (2008).

20. Litvan, I. et al. Clinical research criteria for the diagnosis of progressive supranuclear palsy (SteeleRichardson-Olszewski syndrome): report of the NINDS-SPSP international workshop. Neurology 47, 1-9 (1996).

21. Thanvi, B., Lo, N. \& Robinson, T. Vascular parkinsonism--an important cause of parkinsonism in older people. Age Ageing 34, 114-119 (2005).

22. Bloem, B. R., Grimbergen, Y. A., Cramer, M., Willemsen, M. \& Zwinderman, A. H. Prospective assessment of falls in Parkinson's disease. J. Neurol. 248, 950-8 (2001).

23. Nonnekes, J., Goselink, R., Weerdesteyn, V. \& Bloem, B. R. The retropulsion test: a good evaluation of postural instability in Parkinson's disease? J. Parkinsons. Dis. 5, 43-7 (2015).

24. Venhovens, J., Meulstee, J. \& Verhagen, W. I. M. Ocular and Cervical Vestibular Evoked Myogenic Potentials (VEMPs) in healthy volunteers: the intra-, interobserver, and the test re-test reliability. J. Vestib. Res. 25, 161-7 (2015). 
25. Venhovens, J., Meulstee, J. \& Verhagen, W. Vestibular Evoked Myogenic Potentials (VEMPs) in Central Neurological Disorders. Clin. Neurophysiol. 127, 40-49 (2016).

26. Vanspauwen, R., Wuyts, F. L. \& Van De Heyning, P. H. Validity of a new feedback method for the VEMP test. Acta Otolaryngol. 126, 796-800 (2006).

27. Zapala, D. A. \& Brey, R. H. Clinical experience with the vestibular evoked myogenic potential. J. Am. Acad. Audiol. 15, 198-215 (2004).

28. Tarnutzer, A. A., Fernando, D. P., Lasker, A. G. \& Zee, D. S. How stable is perceived direction of gravity over extended periods in darkness? Exp. Brain Res. 222, 427-436 (2012).

29. Thijs, R. D., Bloem, B. R. \& Dijk, J. G. Falls, faints, fits and funny turns. J. Neurol. 256, 155-167 (2009).

30. de Natale, E. R. et al. Paired neurophysiological and clinical study of the brainstem at different stages of Parkinson's Disease. Clin. Neurophysiol. 126, 1871-8 (2015).

31. de Natale, E. R. et al. Abnormalities of vestibular-evoked myogenic potentials in idiopathic Parkinson's disease are associated with clinical evidence of brainstem involvement. Neurol. Sci. 36, 995-1001 (2015).

32. Pötter-Nerger, M., Reich, M. M., Colebatch, J. G., Deuschl, G. \& Volkmann, J. Differential effect of dopa and subthalamic stimulation on vestibular activity in Parkinson's disease. Mov. Disord. 27, 1268-75 (2012). 


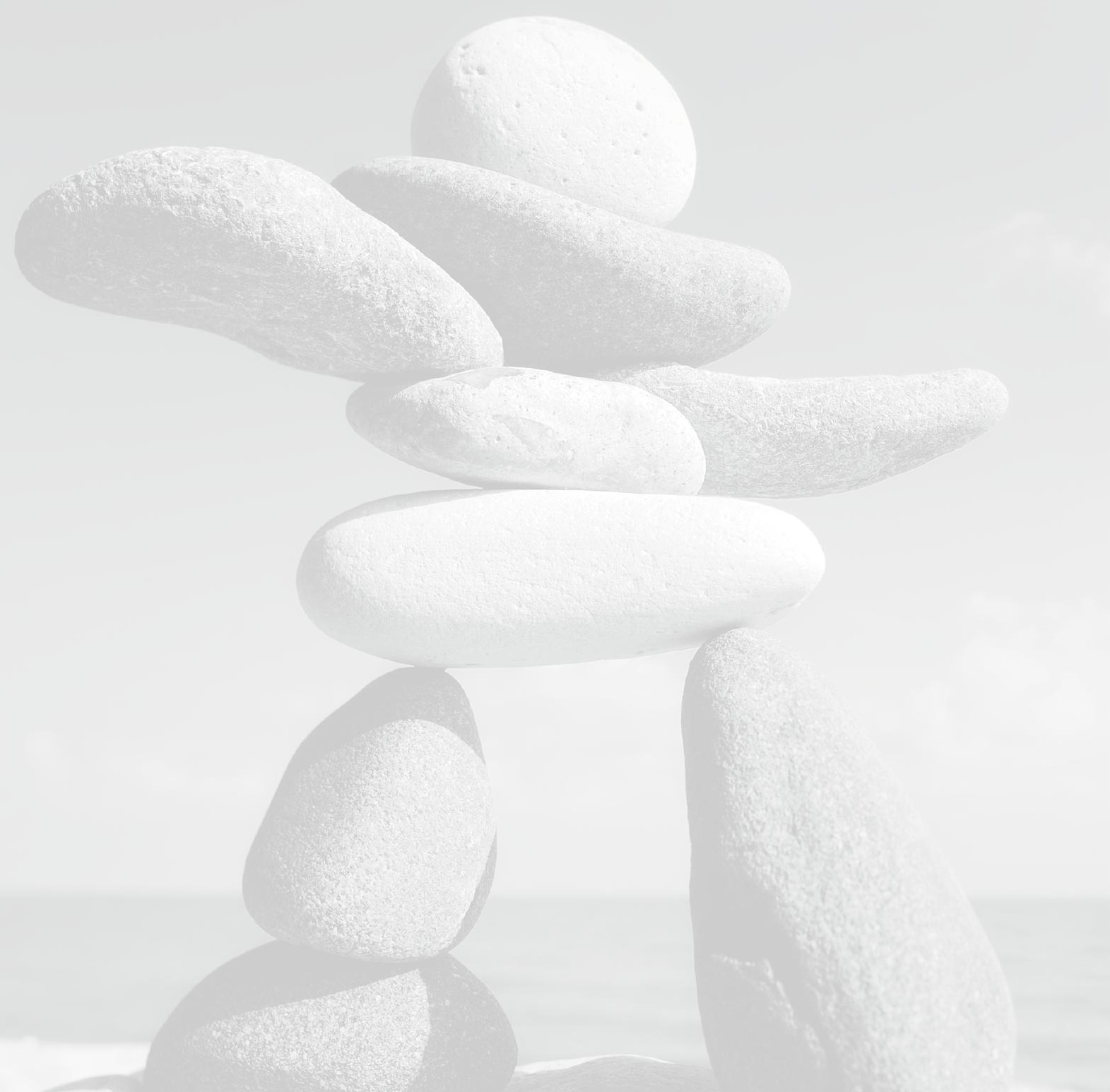




\section{Chapter 6}

Neurovestibular analysis and falls in Parkinson's disease and atypical parkinsonism:

Results from the prospective one-year follow-up study

Venhovens, J., Meulstee, J., Bloem, B. R. \& Verhagen, W. I. M 


\section{Abstract}

\section{Objectives}

Our primary aim was to determine whether neurovestibular laboratory tests can predict future falls in patients with either Parkinson's disease (PD) or atypical parkinsonism (AP).

\section{Methods}

We included 25 healthy subjects, 30 PD patients (median Hoehn and Yahr stage 2.5, range 1-4), and $14 \mathrm{AP}$ patients (5 multiple system atrophy, 3 progressive supranuclear palsy, and 5 vascular parkinsonism) in a case-control study design (all matched for age and gender). At baseline, all subjects underwent clinical neurological and neurotological assessments, cervical and ocular vestibular evoked myogenic potentials (cervical and ocular VEMPs), brainstem auditory evoked potentials (BAEPs), subjective visual vertical measurements (SVV), and video nystagmography (VNG) with caloric and rotary test stimulation. After one year follow-up, all subjects were contacted by telephone for an interview concerning their fall frequency based upon their fall diaries and balance confidence (according to the $\mathrm{ABC}-16$ questionnaire); only one was lost to follow-up (attrition bias of only $1.4 \%)$.

\section{Results}

Cervical and ocular VEMPs combined with clinical tests for postural imbalance predict future fall incidents in both PD and AP groups with a sensitivity of $100 \%$. A positive predictive value (PPV) of $68.2 \%$ was achieved, if only one VEMP test was abnormal, and a PPV of $83.3 \%$ when both VEMP tests were abnormal. The fall frequency both at baseline and after one year was significantly higher and the balance confidence scale (ABC-16) was significantly lower in both the PD and AP groups compared to healthy controls.

\section{Conclusions}

Cervical and ocular VEMP testing can predict the risk of future fall incidents in $\mathrm{PD}$ and $\mathrm{AP}$ patients with postural imbalance in the absence of freezing of gait. Obviously, at this moment we cannot predict whether the knowledge of this increased risk will subsequently result in effective strategies to diminish or even prevent falling. 


\section{Introduction}

Falls are highly prevalent in patients with Parkinson's disease (PD) or atypical parkinsonism (AP). Approximately $70 \%$ of PD patients have at least one fall episode annually ${ }^{1}$. Fall incidents often lead to social isolation which may result in a reduced quality of life, because fall incidents can cause a fear of renewed fall episodes, possibly resulting in a self-imposed restriction of daily activities ${ }^{2-4}$.

We previously showed that vestibular dysfunction is an independent risk factor for the occurrence of falls in PD and AP patients ${ }^{4}$. The results of vestibular tests mainly reflect central neurological vestibular dysfunction, even though these patients usually do not complain of vertigo or dizziness ${ }^{4}$. Patients with PD or AP who had experienced prior falls had more abnormal vestibular test results compared to non-falling patients. After exclusion of the well-established causes of falls (e.g. orthostasis, freezing of gait, cognitive problems and postural instability) $10-18 \%$ of the falling PD and AP patients had vestibular system abnormalities as the only identifiable cause for falling ${ }^{4}$. We therefore concluded that vestibular system dysfunction, as established with neurovestibular laboratory tests, is an independent and relevant risk factor for falling in $\mathrm{PD}$ and $\mathrm{AP}$.

The primary aim of this prospective study was to determine whether neurovestibular laboratory tests have predictive value for the occurrence of future falls in PD and AP patients and, if so, to determine their sensitivity, specificity, and positive predictive values. The secondary aim was to determine the fall frequency and balance confidence in both PD and AP after one year of follow-up, as compared with an age- and gendermatched healthy control group.

\section{Methodology}

\section{Study Participants}

Previously we described the methodology and baseline measurements of our study cohort ${ }^{4}$. Now we present the data after one year follow-up (median 12 months, range 12-14 months). The neurovestibular laboratory tests conducted at baseline were: a) cervical and ocular vestibular evoked myogenic potentials (VEMPs), b) subjective visual vertical (SVV), and c) video nystagmography (VNG) with additional caloricand rotatory chair stimulation. The study was approved by the regional and local medical ethical committee (CMO Arnhem-Nijmegen, the Netherlands, number 2012/393) and was registered as well in the Dutch trial register (Nederlands Trial 
Register, NTR-3928). All volunteers signed an informed consent. Healthy controls and patients did not have a relevant medical history (i.e. no relevant neurological, otological, ophthalmological diseases, and/or absence of moderate-to-severe cognitive problems) with the exception of PD or AP (in combination with a related cerebrovascular disorder in the vascular parkinsonism group). Controls were matched for age and gender with the PD and AP patients. Sixty-nine volunteers were included in the baseline case-control study. Only one patient with multiple system atrophy with predominant parkinsonism (MSA-P) was lost to follow-up, resulting in an attrition bias of only $1.4 \%$.

Sixty-eight volunteers completed the follow-up study; 25 healthy controls (mean age 67, range 42-81, 15 men), $30 \mathrm{PD}$ patients (mean age 70, range 59-81, 26 men, all fulfilling the UK Parkinson's Disease Society Brain Bank criteria ${ }^{5}$, median Hoehn and Yahr stage 2.5, range 1-4), and 13 atypical parkinsonism (AP) patients (mean age 68, range 52-81, 8 men, 5 multiple system atrophy, 3 progressive supranuclear palsy, and 5 vascular parkinsonism). The MSA-P patients all fulfilled the diagnostic criteria for probable MSA-P as proposed in the consensus statement by Gilman ${ }^{6}$. Supranuclear palsy patients (PSP) all fulfilled the NINDS-SPSP criteria for possible $\mathrm{PSP}^{7}$. Vascular parkinsonism patients all fulfilled the criteria of the Winikates and Jankovic vascular rating scale ${ }^{8}$.

\section{Follow-up by telephone interview}

All participants were contacted by telephone for an interview one year after the baseline measurements. At the end of the baseline examinations, they were asked to keep track of their fall incidents during the following year by means of a fall diary. During the telephone interview, they were questioned about their fall frequency during the previous year, their fear of falling according to the ABC-16 questionnaire (16-items activities-specific balance confidence scale), acquired injuries related to fall incidents, and whether they had received medical treatment for such injuries.

\section{Statistical analysis}

The statistical database software SPSS version 23.0 (SPSS inc., USA) was used for statistical analyses. The Shapiro-Wilks test was applied to determine whether parametrical tests were applicable. Because of this test result and due to the limited sample size of our study we had to apply non-parametrical tests for further statistical analyses. The Kruskal-Wallis one-way analysis by ranks test was applied for comparison of the continuous non-parametrically distributed data of the three independent groups (controls, PD, and AP), the Mann-Whitney-U test for a group to group comparison, and a significance level of 5 percent was used for all analyses. 
An ordinal logistic regression analysis was applied for comparison of the categorical variables. We did not perform a multivariate regression analysis of the data due to the limited sample size.

\section{Results}

We refer to table 1 for the PD and AP patients' individual data concerning the oneyear follow-up results in relation to the baseline measurements, and to table 2 for the group characteristics. Both tables were adapted from our baseline study ${ }^{4}$.

From our data in table 2 and additional group-to-group comparisons it may be inferred that PD and AP patients fall statistically significantly more often in comparison to age- and gender-matched healthy control subjects at baseline $\left(\mathbf{P}_{\mathbf{P D}-\text {-controls }}\right.$ $\left.=\mathbf{0 . 0 3 7} ; \mathbf{P}_{\text {AP-controls }}<\mathbf{0 . 0 0 1}\right)$. In addition, the percentage of falling AP patients compared to healthy controls during the follow-up year was statistically significantly higher $\left(\mathrm{P}_{\mathrm{PD} \text {-controls }}=0.571 ; \mathbf{P}_{\text {AP-controls }}=\mathbf{0 . 0 3 4}\right)$. Also, the percentage of falling AP patients was higher than the PD patients, however the difference was only statistically significant at baseline $\left(\mathbf{P}_{\text {baseline-PD-PA }}=\mathbf{0 . 0 3 2} ; \mathrm{P}_{\text {follow-up-PD-PA }}=0.090\right)$. The PD patients and the AP patients have a statistically significantly higher fall frequency at baseline in comparison to controls (Baseline: $\mathbf{P}_{\text {PD-controls }}=\mathbf{0 . 0 3 5} ; \mathbf{P}_{\text {AP-controls }}=\mathbf{0 . 0 0 1}$ ); and both also have a higher fall frequency during follow-up, however it is only the difference between the AP patients and controls that is statistically significant (Follow-up: $\mathrm{P}_{\mathrm{PD}-}$ controls $=0.394 ; \mathbf{P}_{\text {AP-controls }}=\mathbf{0 . 0 3 2}$ ). AP patients have a higher frequency of falling in comparison to the PD patients, however only the baseline measurements were statistically significant $\left(\mathbf{P}_{\text {baseline-PD-PA }}=\mathbf{0 . 0 3 9}\right.$; $\left.\mathrm{P}_{\text {follow-up-PD-PA }}=0.116\right)$. Also, the increase in fall frequency was especially striking in the AP group in comparison to both the PD patients and controls, however the difference is not statistically significant between the groups $(\mathrm{P}=0.164)$. The number of fall incidents did increase in the AP and PD groups when comparing the baseline and follow-up measurements, however the difference is not statistically significant $\left(\mathrm{P}_{\text {baseline-follow-up-AP }}=0.257 ; \mathrm{P}_{\text {baseline-follow-up- } \mathrm{PD}}=\right.$ $0.705)$. The increase in the healthy control group, however, is statistically significant $\left(P_{\text {baseline-follow-up-control }}=\mathbf{0 . 0 4 6}\right)$

The 16-items activities-specific balance confidence scale (ABC-16) differed significantly between the groups in total both at baseline $\left(\mathbf{P}_{\text {total }}=\mathbf{0 . 0 0 6} ; \mathbf{P}_{\mathrm{PD} \text {-controls }}=\mathbf{0 . 0 2 8} ; \mathbf{P}_{\text {AP- }}\right.$ controls $\left.=\mathbf{0 . 0 0 4} ; \mathrm{P}_{\mathrm{PD}-\mathrm{AP}}=0.096\right)$; and during follow-up one year later $\left(\mathbf{P}_{\text {total }}=\mathbf{0 . 0 0 1}\right.$; $P_{\text {PD-controls }}=\mathbf{0 . 0 2 2} ; P_{\text {AP-controls }}=\mathbf{0 . 0 0 1} ; P_{\text {PD-AP }}=\mathbf{0 . 0 1 8}$ ). Moreover, the difference was statistically significant between the groups when only the non-falling patients were considered both at baseline $\left(P_{\text {total }}=\mathbf{0 . 0 1 0} ; P_{\text {PD-controls }}=\mathbf{0 . 0 1 4 ;} \mathbf{P}_{\text {AP-controls }}=\mathbf{0 . 0 0 9}\right.$; 
PART TWO I Chapter 6

Table 1.

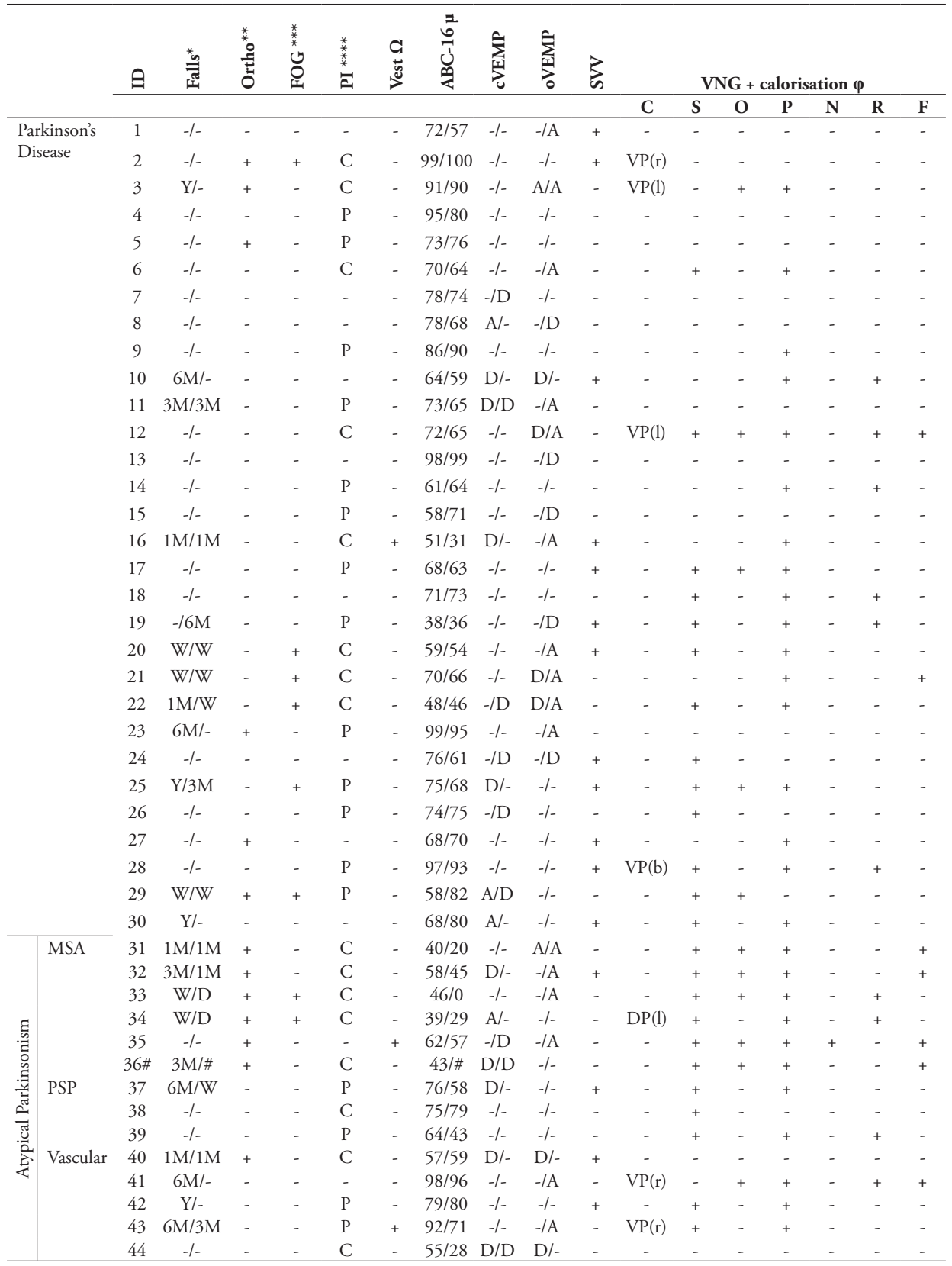

Adapted from Venhovens et al. (Venhovens et al., 2016). Individual clinical characteristics and test results of patients with Parkinson's disease and atypical Parkinsonism. * Number of fall episodes during the last year (fall frequency at baseline / fall frequency during one-year follow-up; Y: once a year; 6M: once every six months; 3M: once every three months; $1 \mathrm{M}$ : monthly; W: weekly; D: daily). Orthostasis (ortho). ${ }^{* * *}$ Freezing of gait (FOG). ${ }^{* * * *}$ Postural imbalance, PI (C: complete imbalance on pull testing without unaided recovery of balance; P: partial imbalance on pull testing with unaided recovery of balance requiring 2 or more backward steps). $\Omega$ Abnormalities during clinical bedside neurovestibular testing. $\mu$ ABC-16 questionnaire (16-items specific confidence of balance scale at baseline / ABC-16 score after one-year follow-up). Evoked potentials: Right/Left responses; A: absent response; D: delayed response; -: normal response. $\varphi$ Videonystagmography (VNG) and calorisation results; C(alorisation; VP: vestibular paresis (left/right/bilateral); DP: directional preponderance (left/right/bilateral)); $\mathrm{S}$ (accede testing); $\mathrm{O}$ (ptokinetics); smooth $\mathrm{P}$ (ursuit); spontaneous $\mathrm{N}$ (nystagmus) in dark and light conditions; Rotary) chair testing; F(ixation) suppression testing; +: abnormal; -: normal. \# Loss to follow-up.

106 
$\left.\mathrm{P}_{\mathrm{PD}-\mathrm{AP}}=0.116\right)$; and during follow-up one year later $\left(\mathbf{P}_{\text {total }}=\mathbf{0 . 0 0 9} ; \mathbf{P}_{\mathrm{PD} \text {-controls }}=\mathbf{0 . 0 1 0}\right.$; $\left.\mathbf{P}_{\mathrm{AP}-\text { controls }}=\mathbf{0 . 0 0 4} ; \mathrm{P}_{\mathrm{PD}-\mathrm{AP}}=0.081\right)$. However, only the change in ABC-16 scores between the baseline measurements and after one year follow-up was statistically significant in the total group $\left(P_{\text {total }}=\mathbf{0 . 0 2 8} ; \mathbf{P}_{\mathrm{PD} \text {-controls }}=\mathbf{0 . 0 2 2 ;} \mathbf{P}_{\text {AP-controls }}=\mathbf{0 . 0 0 1}\right.$; $P_{\text {PD-AP }}=\mathbf{0 . 0 1 8}$ ). However, the $\mathrm{ABC}-16$ results of the falling patients were similar in the groups. The fall related injuries both at baseline or during follow-up and their treatments did not differ significantly.

\section{Discussion}

Laboratory examinations, and especially the vestibular evoked myogenic measurements, VEMPs (an abnormal VEMP result, defined as having at least one abnormal result at both the cervical and/or ocular VEMP tests combined) have a sensitivity of $100 \%$ to predict the occurrence of falls, at the cost of a low PPV of 46.8\% (15 of the 32 patients will fall during follow-up). The presence of freezing of gait is also a strong predictor for the occurrence of falls (seven out of eight patients will fall, yielding a PPV of $87.5 \%$ ). However, the sensitivity for detecting patients at risk for future falls is limited as only seven out of 15 patients will be detected (46.6\%). The sensitivity for detecting patients at risk for falling is very high concerning the clinical testing for the presence of postural instability (100\%), but the PPV is only $46.9 \%$.

Therefore, there is no single clinical or laboratory test (that is independent of clinical tests) with a high positive predictive value and a high sensitivity for detecting patients with a high risk for falling. Both kinds of tests are needed to make a screening test that is useful in clinical practice in order to detect all patients at risk, however to also prevent a large number of false positive results which would lower the diagnostic value.

However, when both tests are used in combination (i.e. to use VEMP testing for additional screening for future fall incidents in those patients who at least have partial postural imbalance) the sensitivity will still remain 100\% (fifteen out of the fifteen patients will be detected); and the PPV will subsequently be $68.2 \%$ when only the ocular or the cervical VEMP is abnormal (15 out of the 22 patients with positive results will fall during the year follow-up), or $83.3 \%$ when both VEMP tests are abnormal ( 5 out of 6 patients with positive results will fall during the follow-up year; with 5 out of 15 falling patients having abnormal results in both VEMP tests). 
Table 2.

Adapted from Venhovens et al. (Venhovens et al., 2016).

\begin{tabular}{|c|c|c|c|c|}
\hline & $\begin{array}{l}\text { Parkinson's } \\
\text { Disease }\end{array}$ & $\begin{array}{l}\text { Atypical } \\
\text { Parkinsonism }\end{array}$ & $\begin{array}{l}\text { Healthy } \\
\text { controles }\end{array}$ & P-value* \\
\hline Number of subjects $(\mathrm{N})$ & 30 & $14^{* *}$ & 25 & 0.054 \\
\hline \multicolumn{5}{|l|}{ Number of falling patients: } \\
\hline - Baseline ( $\mathrm{N}$, percentage) & $11(37)$ & $10(71)$ & $3(12)$ & $<0.001$ \\
\hline - One year follow-up ( $\mathrm{N}$, percentage) & $8(27)$ & $7(54)$ & $5(20)$ & 0.043 \\
\hline \multicolumn{5}{|l|}{ Average number of falls/year (baseline): } \\
\hline - All patients in total (average, SD) & $6,4(15,8)$ & $10,2(18,1)$ & $0,3(0,9)$ & $<0.001$ \\
\hline - Only falling patients (average, SD) & $16,0(22,1)$ & $14,3(20,3)$ & $2,7(1,2)$ & \\
\hline \multicolumn{5}{|l|}{ Average number of falls/year (one-year follow-up): } \\
\hline - All patients in total (average, SD) & $7,7(3,3)$ & $63,2(37,4)$ & $0,9(0,5)$ & 0.032 \\
\hline - Only falling patients (average, SD) & $28,8(8,8)$ & $117,4(64,2)$ & $4,4(2,0)$ & \\
\hline \multicolumn{5}{|l|}{ Change in number falls/year (absolute, percentage) } \\
\hline - All patients in total (absolute, percentage) & $+1,3(+21,1)$ & $+53,0(+518,7)$ & $+0,6(+175,0)$ & 0.164 \\
\hline - Only falling patients (absolute, percentage) & $+9,8(+51,3)$ & $+103,1(+721,0)$ & $+1,7(+65,0)$ & \\
\hline \multicolumn{5}{|l|}{ ABC-16 fear of falling, Baseline: } \\
\hline - All patients in total (average, SD) & $72,9(2,8)$ & $64,7(5,1)$ & $82,3(3,6)$ & 0.006 \\
\hline - Only falling patients (average, SD) & $59,0(4,6)$ & $57,9(6,4)$ & $60,8(9,4)$ & 0.914 \\
\hline - Only non-falling patients (average, SD) & $78,0(2,8)$ & $72,2(6,3)$ & $87,7(3,0)$ & 0.010 \\
\hline \multicolumn{5}{|l|}{ ABC-16 fear of falling, Follow-up after one-year: } \\
\hline - All patients in total (average, SD) & $70,5(3,1)$ & $51,2(7,5)$ & $80,3(3,6)$ & 0.001 \\
\hline - Only falling patients (average, SD) & $56,0(6,2)$ & $38,8(8,4)$ & $53,8(7,9)$ & 0.190 \\
\hline - Only non-falling patients (average, SD) & $75,8(2,9)$ & $63,8(10,5)$ & $87,0(2,4)$ & 0.009 \\
\hline \multicolumn{5}{|l|}{ Change in $\mathrm{ABC}-16$ : } \\
\hline - All patients in total (absolute, percentage) & $-2,4(-3,3)$ & $-13,5(-20,9)$ & $-2,0(-2,4)$ & 0.028 \\
\hline - Only falling patients (absolute, percentage) & $-3,0(-5,1)$ & $-19,1(-33,0)$ & $-7,0(-11,5)$ & 0.156 \\
\hline - Only non-falling patients (absolute, percentage) & $-2,2(-2,8)$ & $-8,4(-11,6)$ & $-0,7(-0,8)$ & 0.506 \\
\hline \multicolumn{5}{|l|}{ Fall injury, Baseline (N, percentage): } \\
\hline - No injury & $1(9)$ & $1(10)$ & $0(0)$ & 0.714 \\
\hline - Minor (e.g. cuts and bruises) & $9(82)$ & $8(80)$ & $2(67)$ & \\
\hline - Intermediate (e.g. simple fractures) & $1(9)$ & $0(0)$ & $1(33)$ & \\
\hline - Severe (e.g. fractures requiring surgery) & $0(0)$ & $1(10)$ & $0(0)$ & \\
\hline \multicolumn{5}{|l|}{ Fall injury, Follow-up after one-year (N, percentage): } \\
\hline - No injury & $0(0)$ & $0(0)$ & $1(20)$ & 0.414 \\
\hline - Minor (e.g. cuts and bruises) & $7(88)$ & $6(86)$ & $3(60)$ & \\
\hline - Intermediate (e.g. simple fractures) & $0(0)$ & $1(14)$ & $0(0)$ & \\
\hline - Severe (e.g. fractures requiring surgery) & $1(12)$ & $0(0)$ & $1(20)$ & \\
\hline \multicolumn{5}{|l|}{ Treatment, Baseline (N, percentage): } \\
\hline - No treatment necessary & $2(18)$ & $1(10)$ & $0(0)$ & 0.714 \\
\hline - Self-treatment & $6(55)$ & $7(70)$ & $2(67)$ & \\
\hline - Outpatient doctor's treatment & $2(18)$ & $1(10)$ & $1(33)$ & \\
\hline - Hospital admission (no surgery) & $1(9)$ & $0(0)$ & $0(0)$ & \\
\hline - Hospital admission for surgery & $0(0)$ & $1(10)$ & $0(0)$ & \\
\hline \multicolumn{5}{|l|}{ Treatment, One-year follow-up (N, percentage): } \\
\hline - No treatment necessary & $0(0)$ & $0(0)$ & $1(20)$ & 0.427 \\
\hline - Self-treatment & $7(88)$ & $5(72)$ & $2(40)$ & \\
\hline - Outpatient doctor's treatment & $0(0)$ & $1(14)$ & $1(20)$ & \\
\hline - Hospital admission (no surgery) & $0(0)$ & $1(14)$ & $0(0)$ & \\
\hline - Hospital admission for surgery & $1(12)$ & $0(0)$ & $1(20)$ & \\
\hline
\end{tabular}

Group characteristics and comparison between the groups concerning the different test results in the Parkinson's disease, atypical Parkinsonism, and healthy control groups. * The P-value is calculated by means of an ordinal regression calculation (in the categorical variables) and the Kruskal-Wallis one-way analysis of variance by ranks test (in the continuously distributed, independent, and nonparametrical variables). A significance level of 5 percent (i.e. P-value $\leq 0.05$ ) was adopted for each analysis and significant $\mathrm{P}$-value results are printed in bold. ${ }^{* *}$ Fourteen patients completed the baseline examinations and one patient was lossed to follow-up. 
Cervical and ocular VEMP testing combined with the clinical evaluation for postural instability gives additional information concerning the future fall risk of PD and AP patients compared to clinical evaluation of postural instability alone. However, the presence of freezing of gait is such a strong predictor for future falls in both PD and AP patients (PPV 87.5\%; all the falling patients with freezing of gait also had abnormal VEMPs and postural instability) that VEMP testing in these patients does not have any additional value. Therefore, cervical and ocular VEMP testing seems to give additional information concerning the future fall risk in selected PD and AP patients (those patients who have postural instability in the absence of freezing of gait). However, one could speculate whether this additional information (possible increase of the PPV from $46.9 \%$ to $68.2-83.3 \%$ ) will aid in guiding future fall prevention therapies (for instance through physical therapy) as $\mathrm{PD}$ and AP patients with postural imbalance already have a high risk for falling. Recently, a practical consensus-based overview concerning the risk factors and management of falls in PD was published emphasizing the multifaceted origin of the falls and the need for a personalized approach?

Decreasing the risk of falling is important, as fall incidents will result in a lowered subjective balance confidence (as can be concluded from the data in table 2.), secondarily resulting in self-imposed restrictions in daily life, ultimately leading to social isolation ${ }^{2,3}$. Moreover, patients with parkinsonism are more at risk for fall-related injuries, such as hip fractures secondarily leading to a higher morbidity, mortality, and health care costs in comparison to individuals without Parkinsonism ${ }^{3,10}$.

Our study also has some important limitations, which we also mentioned earlier ${ }^{4}$. The first limitation relates to the small sample size; this was explained by the strict inclusion/exclusion criteria and the lengthy nature of the neurovestibular testing, which is especially demanding for elderly and AP patients. Therefore, the results from this study need to be interpreted cautiously and as hypothesis-generating for further research, especially in the heterogeneous AP group. We advocate further research concerning the additional value of VEMP testing for predicting the risk of falling in larger PD and AP groups. The second limitation is, that the volunteers may have had a bias concerning the recollection of their falling incidents. We tried to overcome this issue by asking the volunteers to keep track of their fall incidents by keeping personalized fall diaries. However, mal-compliance could bias the results possibly leading to an underestimation of the true fall incidence. Therefore, we questioned the volunteers about their fall frequency instead of the absolute number of falls, to minimize the effects of mal-compliance and recollection bias. 
To conclude, we found a high prevalence in the number of falling patients and fall incidents in our follow-up study after one year in both PD and AP patients. After one year, especially the frequency of the fall incidents in the AP group increased in comparison to the PD and control group, which was not statistically significant (probably as a result of the small group size) as the other groups did also show a less pronounced increase in the number of fall incidents. The risk of future falls in $\mathrm{PD}$ and AP patients can be predicted better when patients with postural imbalance on clinical testing are additionally tested by means of cervical and ocular VEMP testing (with the exclusion of patients with freezing of gait). However, it remains unclear if the increase in future fall risk (possible PPV increase from 46.9\% to 68.2-83.3\%) will aid in the different utilization of fall prevention strategies as $\mathrm{PD}$ and $\mathrm{AP}$ patients with postural imbalance already have a high risk of falling. 


\section{References}

1. Bloem, B. R., Steijns, J. A. G. \& Smits-Engelsman, B. C. An update on falls. Curr. Opin. Neurol. 16, 15-26 (2003).

2. Bloem, B. \& Bhatia, K. in Clinical disorders of balance, posture and gait. (ed. Bronstein AM, Brandt T, Nutt JG, W. M.) 173-206 (Arnold: London, 2004).

3. Grimbergen, Y. A. M., Munneke, M. \& Bloem, B. R. Falls in Parkinson's disease. Curr. Opin. Neurol. 17, 405-415 (2004).

4. Venhovens, J., Meulstee, J., Bloem, B. R. \& Verhagen, W. I. M. Neurovestibular analysis and falls in Parkinson's disease and atypical parkinsonism. Eur. J. Eur. J. Neurosci. 43, 1636-46 (2016).

5. Jankovic, J. Parkinson's disease: clinical features and diagnosis. J. Neurol. Neurosurg. Psychiatry 79, 368-76 (2007).

6. Gilman, S. et al. Second consensus statement on the diagnosis of multiple system atrophy. Neurology 71, 670-676 (2008).

7. Litvan, I. et al. Clinical research criteria for the diagnosis of progressive supranuclear palsy (SteeleRichardson-Olszewski syndrome): report of the NINDS-SPSP international workshop. Neurology 47, 1-9 (1996).

8. Thanvi, B., Lo, N. \& Robinson, T. Vascular parkinsonism--an important cause of parkinsonism in older people. Age Ageing 34, 114-119 (2005).

9. van der Marck, M. A. et al. Consensus-based clinical practice recommendations for the examination and management of falls in patients with Parkinson's disease. Parkinsonism Relat. Disord. 20, 3609 (2014).

10. Pressley, J. C. et al. The impact of comorbid disease and injuries on resource use and expenditures in parkinsonism. Neurology 60, 87-93 (2003). 


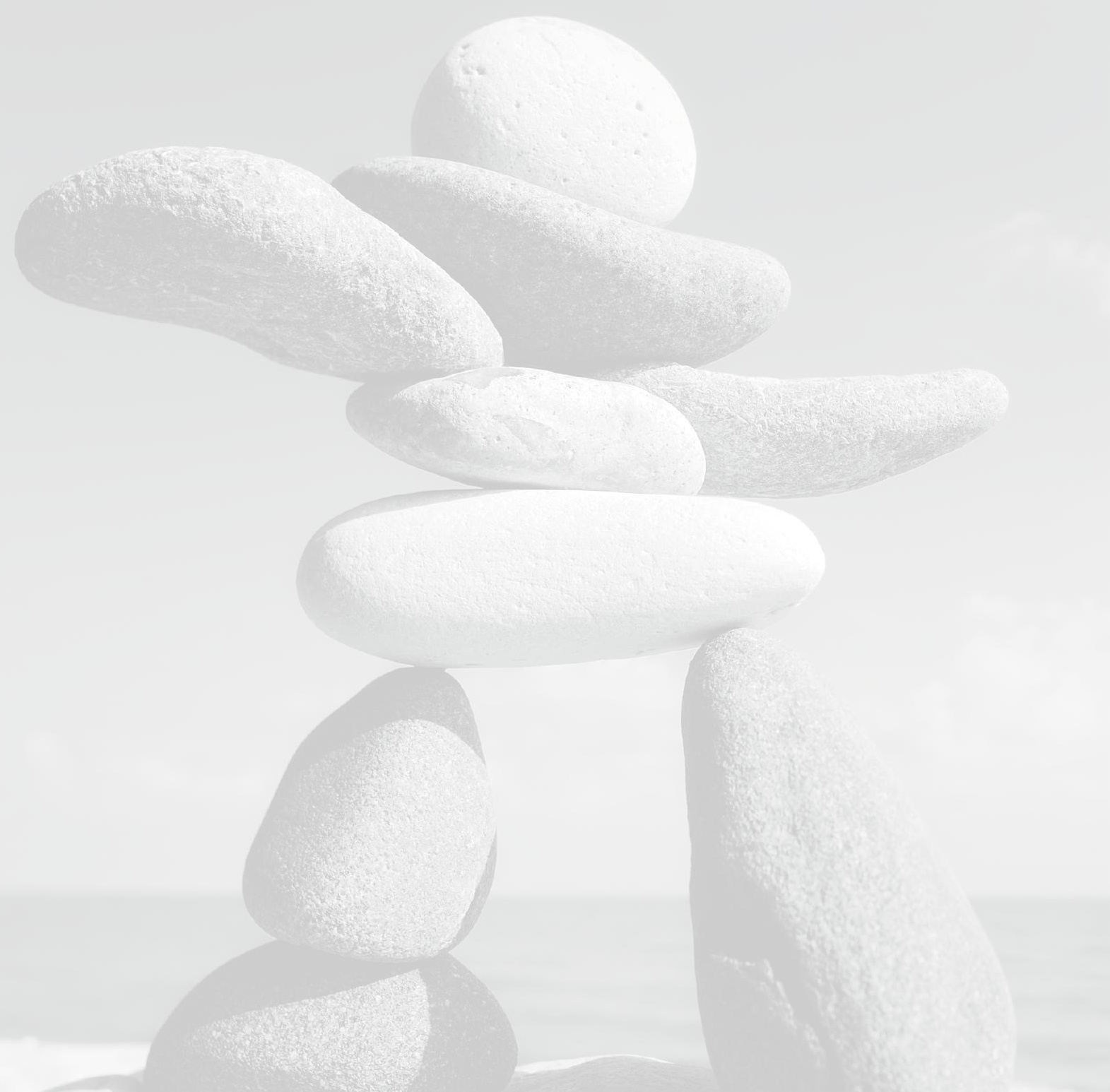




\section{Chapter 7}

Summary, general discussion, and perspectives for future research 
As mentioned earlier, this thesis is divided into two parts which will be discussed separately. The first part covers technical and practical issues of neurovestibular laboratory examinations, with an emphasis on vestibular evoked myogenic potentials (VEMP) and static subjective visual vertical testing (SVV). The second part offers a detailed overview of a study concerning neurovestibular analysis in persons with Parkinson's disease (PD) or atypical parkinsonism (AP), both at baseline and after one-year follow-up. Each part begins with a brief summary of the respective chapter(s), followed by a general discussion and some thoughts on the perspectives for future research.

\section{PART ONE}

\section{Neurovestibular laboratory testing - practical and technical issues}

\section{Vestibular evoked myogenic potentials (VEMP)}

\section{Summary chapters 2 and 3}

Over the past two decades, vestibular evoked myogenic potential (VEMP) testing has come into use as a test suitable for detailed neurophysiological assessment of both the central and peripheral vestibular system. VEMP testing can be divided into a cervical and an ocular response. The cervical VEMP test assesses the integrity of the vestibulo-spinal reflex arc beginning at the saccular afferents to ultimately evoke an inhibitory motor response in the ipsilateral sternocleidomastoid muscle (by subsequently travelling through the ipsilateral inferior vestibular nerve, vestibular nuclei, medial vestibulospinal tract, cervical motor neurons, and the accessory nerve $)^{1-5}$. The ocular VEMP test assesses the integrity of the vestibuloocular reflex arc most likely beginning at the utricular afferents (however, it is debated extensively in literature whether the precise origin is predominately the utriculus or a combination of both the utriculus and the sacculus) to ultimately evoke an excitatory motor response in the contralateral inferior oblique external eye muscle (by subsequently travelling through the ipsilateral superior vestibular nerve, vestibular nuclei, medial longitudinal fasciculus, and contralateral oculomotor nucleus and nerve) ${ }^{1-10}$. We refer to figure 1 , chapter 2 , for a schematic representation of the complex neurophysiological pathways concerning the vestibulo-ocular and vestibulo-spinal reflex arcs and to figure 2 , chapter 2 , for an example of typical normal VEMP responses ${ }^{5}$. Chapter 2 gives a detailed overview 
concerning VEMP abnormalities in different central neurological disorders, the interpretation of these results, and possible clinical applications. This was achieved through critical assessment of the available literature; the level of diagnostic evidence was subsequently estimated. The general level of diagnostic evidence according to the Oxford centre of evidence-based medicine mainly exists of level 4 evidence with exceptions reaching a maximum of level $2 \mathrm{~b}$ evidence (for Multiple Sclerosis, vestibular schwannomas, PD, and vertebrobasilar stroke) leaving the total diagnostic evidence concerning VEMPs in central neurological disorders to be limited at this moment. However, VEMP abnormalities are seen rather often in central neurological disorders and careful interpretation of these abnormalities may give important localizing information, but mostly are not disease specific. We refer to table 1, chapter 2, for a detailed description of VEMP abnormalities at different anatomical localizations ${ }^{5}$. In Chapter $\mathbf{3}$ a prospective ocular and cervical VEMP study is described concerning the intra-, interobserver, and the test re-test reliability in twenty healthy volunteers. The intra- and interobserver reliability, which is an important aspect of clinical VEMP judgement, concerning the three experienced clinical neurophysiologists is excellent for both ocular and cervical VEMP studies. The group results concerning the first test and the re-test were similar and did not differ significantly. However, the individual results showed relevant variability. Despite this individual variability, the results of all volunteers remained within normal limits. The raw ocular and cervical VEMP peak-to-peak amplitudes were the most reliable test re-test parameters, followed by cervical VEMP latencies. The ocular VEMP latencies showed a poor test re-test reliability ${ }^{11}$.

VEMP abnormalities are seen rather often in patients with either peripheral or central neurovestibular disorders, as well as in other central neurological disorders ${ }^{5,12}$. Careful interpretation of these abnormalities may give important localizing information concerning the side and the origin of the lesions within the central or peripheral vestibular system, which is the main strength of VEMP testing. However, VEMP testing performed for suspected peripheral vestibular disorders should be interpreted with great care when the patient has a concomitant central neurological disorder and vice versa. Chapter 2 gives additional information regarding the anatomical localization of VEMP abnormalities. In addition, I have to stress that the level of diagnostic evidence according to the Oxford centre of evidence-based medicine is currently rather limited to a general level 4 evidence with exceptions reaching a maximum of level $2 \mathrm{~b}$ evidence (for Multiple Sclerosis, vestibular schwannomas, PD, and vertebrobasilar stroke). Therefore, in my opinion the primary indication for 
VEMP testing in relationship to neurovestibular disorders should at this moment be patients whose neurovestibular complaints cannot be reliably localized to or within the central or peripheral neurovestibular system clinically, neither with (video) nystagmography and additional caloric stimulation, nor with brain magnetic resonance imaging (MRI) ${ }^{5}$. There is however, one exception being the diagnostic work-up of a patient with a suspected superior semicircular canal dehiscence syndrome (SCDS), in which decreased ipsilateral cervical VEMP stimulation thresholds and especially increased contralateral ocular VEMP amplitudes are a very sensitive sign, which can help in identifying the pathological side. The main cause of this phenomenon is supposed to be the increased sound and pressure sensitivity of the ipsilateral labyrinth in comparison to the contralateral side $\mathrm{e}^{13,14}$.

In general, VEMP abnormalities are not considered to be disease specific and thus do not give additional information concerning the nature of the lesion. However, one could speculate whether careful interpretation of the VEMP abnormalities can give insight into the pathophysiological hallmark of the disorder (whether the lesion is primary demyelinating, axonal, or a combination of both), similar to for example peripheral neuropathies. Demyelinating peripheral neuropathies are defined by a severe reduction in nerve conduction velocities, conduction blocks, increased temporal dispersion, severely delayed F-waves, and/or severely delayed distal nerve conduction latencies. Moreover, axonal peripheral neuropathies are defined by reduced amplitudes in the presence of relatively spared nerve conduction velocities ${ }^{15,16}$.

To our knowledge, there are currently no reliable neurophysiological criteria to differentiate primary demyelinating or axonal disorders in relationship to nerve conduction studies by means of evoked potentials for central nervous system disorders. For example, optic neuritis in Multiple Sclerosis (which pathophysiologically has a primary demyelinating hallmark) typically causes asymmetric severe latency delays of the ipsilateral visual evoked potential (VEP) measurements with relatively spared amplitudes. Moreover, optic neuropathies caused by external compression, ischemia, metabolic disturbances, and neurodegenerative disorders tend to cause less pronounced latency delays and possibly a larger reduction in amplitudes in comparison to VEP abnormalities caused by optic neuritis ${ }^{17,18}$. However, brainstem auditory evoked potential (BAEP) abnormalities in Multiple Sclerosis patients are very aspecific, mainly causing peak IV and $\mathrm{V}$ abnormalities followed by latency prolongation of the I-V interpeak and especially the III-V interpeak latencies. The abnormalities described above, however, are not typical for demyelinating disorders and can be found in numerous other disorders, for instance brainstem gliomas, spinocerebellar degeneration, and hydrocephalus ${ }^{19}$. The VEMP results in Multiple Sclerosis patients 
mainly show increased latencies followed by absent responses. However, delayed latencies were also reported in patients with Alzheimer's disease, in patients with migraine, and in a few patients with vertebrobasilar stroke (most had absent responses followed by diminished amplitudes) 5 .

To conclude on a hypothetical and purely speculative basis, on could argue, based upon all the arguments mentioned above, that severe asymmetrically prolonged VEMP latencies (more than 130 percent of the upper limit of normal) with relatively spared amplitudes and wave morphology may be caused by demyelinating lesions (analogous to VEP abnormalities in optic neuritis and nerve conduction abnormalities in demyelinating peripheral neuropathies). However, one could also state the opposite: that evoked potential measurements (and especially the BAEP and VEMP) do at this moment not allow this pathophysiological differentiation concerning the underlying aetiology based upon the numerous BAEP and VEMP case-reports in different disorders, which show that delayed latencies can also be found in numerous other conditions, which do not have a primary demyelinating hallmark (e.g. vertebrobasilar stroke and migraine). However, these reports do not contain detailed descriptions concerning the extend of the latency prolongation, which is expected to be more severe in demyelinating lesions compared to axonal lesions. Further clarification concerning this pathophysiological differentiation based upon evoked potential measurements for central neurological disorders seems interesting for further research. Not only could this pathophysiological differentiation be helpful diagnostically, if possible at all this differentiation could aid in unravelling the underlying pathophysiology of different central nervous system disorders (e.g. neurodegenerative disorders) and to follow their progression over time. To begin with, a similar studydesign which was used in the peripheral polyneuropathy study by Tankisi et al. ${ }^{15}$, comparing amplitudes and nerve conduction velocities in different medical disorders with a well-known pathophysiological hallmark, could be followed (however, adapted for VEMP and BAEP measurements in central nervous system disorders).

In Chapter 3, an important aspect of clinical VEMP judgement is described, being the intra- and interrater reliability, which was excellent in our study ${ }^{11}$. This study also demonstrates that experienced clinical neurophysiologists / neurologists with limited experience in the judgement of individual VEMP recordings could be reliably trained to judge individual examinations in a very short time ( 45 minutes).

The VEMP test re-test study demonstrates an individual variability, however all results remained within normal limits, so this does not seem to be relevant for healthy subjects. One could speculate whether this is also the case for patients with abnormal 
test results (whether their test results remained abnormal if these patients were retested). The ocular and especially the cervical VEMP peak-to-peak amplitudes seem to be the most reliable test re-test parameters, followed by the cervical VEMP latencies. The ocular VEMP latencies on the other hand show a poor test re-test reliability. Standardization of the cervical VEMP amplitudes by means of standardizing the level of Sternocleidomastoid muscle contraction during the measurements, also is an important aspect of performing VEMP testing (for instance with a pressure gauge to assure that the pre-defined target level of force is applied consistently, as described by Vanspauwen et al. ${ }^{20,21}$ ). An alternative method for standardization of the cervical VEMP amplitudes is to correct them by using the rectified mean pre-stimulus electromyographic background signal. Unfortunately, at this moment well-defined methods for standardization of the ocular VEMP amplitudes do not exist. Nguyen et $a l .^{22}$ described an interesting method which they used for standardization of their ocular VEMP amplitudes, which to our knowledge has not been studied extensively in literature elsewhere. The authors used vertical saccades to correct their electrode placement. The recording electrodes were replaced when the inter eye amplitude caused by the vertical saccades was greater than 25 percent. The ocular VEMP test re-test study by Nguyen et al. ${ }^{22}$ showed a more reliable ocular VEMP amplitude in comparison to our study (moderate-to-good reliability in our study compared to an excellent reliability in the study by $\mathrm{Nguyen} \mathrm{et} \mathrm{al.} .^{22}$ ). However, unfortunately the overall poor reliability of the ocular VEMP latencies did not improve with these standardization procedures. These standardization methods for the ocular VEMP are possibly effective and should certainly be investigated further.

\section{Static subjective visual vertical (SVV)}

\section{Summary chapter 4}

Graviception is the perception of a person's orientation relative to the gravitational force, which can be measured by means of the (static) subjective visual vertical (SVV). These graviceptive pathways integrate multimodal sensory input (i.e. vestibular, proprioceptive, and visual input) and subsequently estimate the relative position of one's body with respect to the absolute vertical: the gravitational field of the Earth ${ }^{23-27}$. The static SVV results are almost exclusively determined by the otolith function and balance, as these are the main receptors in the peripheral vestibular system sensitive to gravitational forces (i.e. linear accelerations). However, neurovestibular lesions located more proximally both in the peripheral 
and central vestibular system can obviously also cause abnormal static SVV results by disrupting the corresponding gravitational pathways secondarily resulting in an otolithic imbalance. Static SVV abnormalities for instance are a sensitive sign of brainstem dysfunction ${ }^{25,28}$. We refer to chapter 4 concerning the static SVV testing methodology. A technical prospective static SVV study in 44 healthy volunteers concerning the effects of different preset angle deviations and the test re-test variability is described in chapter 4 . The static SVV results were significantly biased toward the side of the preset angle (Wilcoxon test, $\mathrm{P} \leq 0.001$ ), however not by the preset angle deviation itself (when deviations of 10, 20 and 30 degrees were compared). The test re-test results of the healthy volunteers were stable at a group level without significant differences, however individually they showed relevant variability with an overall poor reliability. However, all individual results remained within normal limits. The last aim of this study was to explore and further construct a robust static SVV testing protocol incorporating both monocular and binocular measurements.

The final aim of the research described in Chapter $\mathbf{4}$ was to explore and construct a robust static SVV testing protocol incorporating both binocular and monocular measurements. The literature is severely hampered by the lack of a uniform testing protocol, resulting in a marked heterogeneity, which makes generalization of SVV findings in literature very difficult. At this moment, most studies in literature only perform binocular measurements, however the performance of both binocular and monocular measurements combined, gives additional information concerning the underlying localization of the lesion. Excellent research performed by Dieterich and Brandt $25,28-30$ showed that $94 \%$ of 111 patients with acute brainstem infarctions have a direction specific pathological binocular static SVV tilt. The binocular static SVV tilt is ipsiversive in peripheral unilateral vestibular disorders or pontomedullary lesions below the crossing of the graviceptive pathways. The binocular static SVV tilt is contraversive in pontomesencephalic brainstem lesions and indicates involvement of the medial longitudinal fasciculus (MLF), interstitial nucleus of Cajal (INC), and/or the rostral interstitial nucleus of the MLF. However, about $64 \%$ of the patients with lesions in the vestibular subnuclei of the thalamus and $44 \%$ of the patients with lesions in the vestibular cortex (i.e. insular, superior temporal, and transverse temporal gyri) too, showed binocular pathologic static SVV tilts, which mainly were contraversive and some were ipsiversive. Yet, it is not only central or peripheral neurovestibular disorders that cause pathologic deviations of the static SVV tilt. Pathologic static SVV tilts also occurred in $67 \%$ of the patients with vertical external eye muscle paresis of 
peripheral origin due to ipsilateral third and fourth cranial nerve palsies. The pathologic static SVV tilts, however, were only monocular and confined to the paretic eye in the acute stage up till three months. Thereafter, the monocular static SVV tilt gradually transferred to the non-paretic eye as a result of compensation mechanisms ${ }^{29}$. The main difference between the pathologic static SVV tilts in supranuclear and infranuclear ocular motor disorders, is that infranuclear abnormalities are strictly confined to the paretic eye in the acute setting and non-paretic eye in the chronic phase after three months. Therefore, binocular static SVV tilts were normal in all patients with infranuclear ocular motor disorders in contrast with supranuclear lesions which were abnormal in $94 \%$ of the brainstem strokes, all showing pathological binocular SVV tilts. Also, bilateral fourth cranial nerve palsies could be localized by means of static SVV testing, which showed bilateral pathologic monocular tilts with an opposite direction and normal binocular static SVV tilts. Moreover, pathologic SVV tilts in supranuclear lesions are always directed towards the same side 25,29 . Therefore, additional static SVV testing incorporating both monocular and binocular measurements gives additional important diagnostic information.

We also demonstrated that static SVV tilts are biased in the direction of the preset angle, but are not influenced by the deviation of the preset angle (for deviations larger than 10 degrees). Our findings are in concordance with earlier studies ${ }^{24,31}$, however preset angle deviations smaller than 8 degrees had less influence on the final static SVV results. In our study and in literature pathologic static SVV tilts between the 10 and 20 degrees were frequently encountered. Therefore, I would advise to use a preset angle deviation of 20 degrees and to divide counter-clockwise and clockwise preset angles equally when performing repeated measurements (because of the bias of the static SVV result in the direction of the preset angle). Both of the recommendations were incorporated in the final static SVV testing protocol, as well as the combination of monocular and binocular measurements.

Analogous to VEMP testing, static SVV testing in healthy volunteers also showed an individual test re-test variability, which was stable at a group level without significant differences. All the results of the healthy volunteers, however, remained within normal limits. One could wonder, if the same is true for patients with pathologic SVV tilts. However, pathologic SVV tilts tend to normalize over time, for instance in brainstem strokes $s^{25,28}$ and to gradually transfer from the paretic eye in the acute phase to the nonparetic eye after a time period of three months in infranuclear ocular motor disorders ${ }^{29}$. We would advise further research concerning the test re-test variability in patients with pathological static SVV tilts, but to keep the time between the tests very short (for instance a few days at the most) because of the dynamic nature of the pathological static SVV tilts. 
The application of static SVV testing outside specialized tertiary dizziness clinics is also hampered by the high costs of the specialized equipment. Interestingly, Zwergal et al..$^{32}$ studied a low-cost, easily performed, and reliable bedside static SVV test by using a selfmade modified bucket, see figure 1 . The conversion of the bucket takes less than one hour and costs less than 5 dollars. The authors compared the bucket-test with conventional static SVV testing by using a hemispheric dome and they found an excellent inter- and intra-test reliability (Pearson correlation coefficients respectively 0.89-0.90 and 0.92) both in healthy subjects and in patients with acute neurovestibular disorders. Brodsky et al. ${ }^{33}$ recently repeated the bucket version of the static SVV test in paediatric patients with suspected neurovestibular disorders, however the authors modified the original bucket test by using a smartphone with a subjective vertical application which costs about 18 dollars. The authors also compared the smartphonebased bucket version of the static SVV test with conventional static SVV testing while using a hemispheric dome and found a moderate correlation (Pearson correlation coefficient 0.43 ). However, despite the moderate correlation with conventional static SVV testing smartphone-based bucket static SVV testing seems to be a useful screening test for acute neurovestibular disorders with a sensitivity of $75 \%$, specificity of $100 \%$, positive predictive value of $100 \%$, and negative predictive value of $92 \%$. However, as promising as these new methods seem, further research with additional comparisons of the (smartphone-assisted) bucket version with the conventional static SVV test is needed in larger groups in order to confirm the earlier research reports.
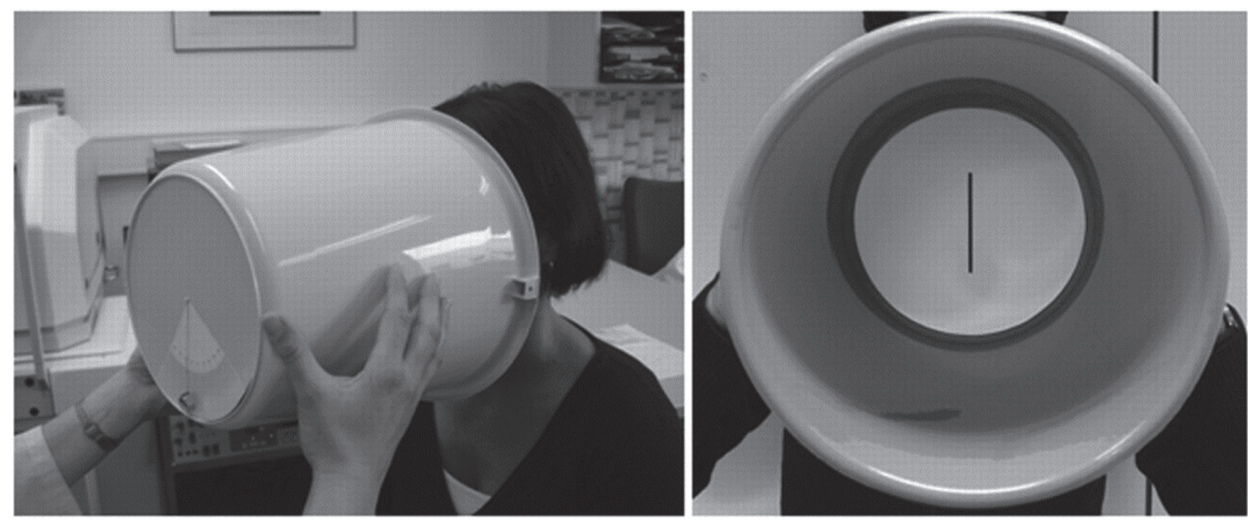

Figure 1.

Bucket version of the SVV test, adapted from Zwergal et al. (2009) $)^{32}$ 


\section{PART TWO}

\section{Neurovestibular analysis and falls in Parkinson's disease and atypical parkinsonism. A baseline study and one-year follow-up study}

\section{Summary chapter 5}

Recurrent falls are a major problem in PD patients, and even more so in patients with AP. About $50 \%$ of the PD patients have recurrent fall episodes, and approximately $70 \%$ fall (at least once) annually ${ }^{34-37}$. PD patients have a 2.2 -fold increase in fractures overall, and a 3 to 4 -fold increase in hip fractures compared to a healthy age-matched population. Moreover, PD patients with hip fractures have higher overall mortality rates, and higher rates of surgical and post-operative complications $^{38}$. Recently, a practical consensus-based overview concerning the risk factors for falling in PD patients was published ${ }^{39}$. However, vestibular function abnormalities were not addressed as an individual risk factor, which is surprising as the vestibular system is one of the key systems in maintaining balance by integrating multimodal sensory information (i.e. vestibular, proprioceptive, and visual sensory input) and by secondarily adjusting the outgoing motor response by means of the vestibulospinal reflexes ${ }^{37}$. Chapter 5 shows the results of a casecontrol study in which the vestibular system function in 25 healthy age-matched volunteers, $30 \mathrm{PD}$, and $14 \mathrm{AP}$ patients (6 patients with multiple system atrophy, 3 with progressive supranuclear palsy, and 5 with vascular parkinsonism) was systematically investigated and compared to an age and gender-matched healthy control group ${ }^{37}$. Ninety percent of PD patients (27 of 30 ) and $100 \%$ of AP patients (all 14) showed signs of neurovestibular dysfunction, mainly with a central vestibular dysfunction profile, on laboratory examinations (i.e. VEMP, SVV, videonystagmography with additional caloric and rotatory chair stimulation). Moreover, vestibular testing abnormalities were correlated with an increased risk of falling when fallers among $\mathrm{PD}$ and $\mathrm{AP}$ patients were compared with non-fallers $(\mathrm{P} \leq 0.001)$.

Abnormal neurovestibular laboratory function test results are seen remarkably often in both PD and AP patients (90\% of the PD patients and 100\% of the AP patients). About $66 \%$ of the patients with mild Parkinson's disease had abnormal neurovestibular laboratory function test results in comparison to $96 \%$ and $100 \%$ of the patients with moderate and severe Parkinson's disease respectively (however, only 1 patient with 
severe Parkinson's disease was included). Therefore, abnormal neurovestibular laboratory test results are very common in early Parkinson's disease, however, they seem to increase in advancing stages of the disorder. Also, patients with atypical Parkinsonism were clinically more affected in comparison to the patients with Parkinson's disease (respectively Hoehn and Yahr stages $2.9 \pm 0.8$ and $2.3 \pm 0.7$; $\mathrm{P}=$ 0.0152). Persons with vestibular and or other neurological disorders (i.e. other than Parkinson's disease or atypical parkinsonism) in their medical history were excluded from the study. Moreover, none of the patients had relevant actual complaints or signs during the neurological and neuro-otological examinations suggestive of other vestibular disorders. We were obviously not able to exclude all other (central) neurovestibular disorders, mainly because the absence of current cerebral imaging studies. However, we did not have any clues suggestive of other neurovestibular disorders in the absence of relevant current medical complaints or abnormalities during neurological and neurovestibular clinical testing.

These abnormal neurovestibular function test results mainly have a central vestibular dysfunction profile in $78-93 \%$ of the PD and AP patients, whereas only one PD patient $(4 \%)$ had a profile suggestive of a peripheral vestibular disorder (the other vestibular dysfunction profiles were non-localizing). Also, 57-79\% of the AP and PD patients did not have complaints of dizziness or vertigo. However, one can question whether these central neurovestibular laboratory testing abnormalities are truly asymptomatic. Indeed, $10-18 \%$ of the AP and PD patients with falls had neurovestibular testing abnormalities as the only identifiable risk factor for falling (after exclusion of orthostatic hypotension, postural instability, freezing of gait, and moderate-to-severe cognitive problems $)^{37}$. However, we acknowledge that we were not able to exclude all possible and previously reported risk factors for falling in $\mathrm{PD}^{39}$, but we did exclude the most relevant causes. Also, we may have missed additional contributions of, for example, mild orthostatic hypotension which can be variably present during clinical examination, and which can be missed during routine testing ${ }^{40}$. However, neurovestibular testing abnormalities were strongly correlated with an increased risk of falling when $\mathrm{PD}$ and AP patients were compared with non-falling patients $(\mathrm{P} \leq 0.001)$. Therefore, it was concluded that central neurovestibular dysfunction on vestibular laboratory testing is highly prevalent in both PD and AP patients compared to healthy volunteers, and is strongly associated with an increased risk of falling, which has not been reported earlier in the literature ${ }^{37}$. I acknowledge that the studied groups in the study were small, and also had a heterogeneous population (especially in the AP group, where subgroups were small). Therefore, the results must be interpreted cautiously and as hypothesis-generating for future research in larger groups. Moreover, it is currently unknown whether these strongly associated 
neurovestibular laboratory abnormalities are directly associated with falling or a secondary epiphenomenon, for instance as a result of deconditioning. Also, dopaminergic medication does not seem to be the cause of these abnormalities as Pötter-Nerger demonstrated that levodopa may partially decrease vestibular testing abnormalities ${ }^{41,42}$. However, the central vestibular system is one of the key systems for maintaining balance by integrating multimodal sensory input (i.e. the visual, peripheral vestibular, and proprioceptive input) and by subsequently adjusting the outgoing motor response by means of the vestibulospinal reflexes. Therefore, one could argue, purely speculative and based upon theoretical physiological evidence, that vestibular dysfunction is causally linked to falling as a possible cause and not an epiphenomenon.

These central vestibular testing abnormalities in PD patients mostly consisted of abnormal evoked potential test results (VEMPs and BAEPs), which showed significant prolongation of the latencies ( $\mathrm{p} 13, \mathrm{n} 1$, and interpeak III-V latencies) on the symptomatic brainstem side compared to healthy volunteers $(0.003 \leq \mathrm{P} \leq 0.019)$. However, comparison of these latencies at the asymptomatic side of PD patients with those of healthy volunteers, or a side-to-side comparison within PD patients, did not yield significantly different results, with the exception of the p13 cervical VEMP latency side-to-side comparison $(\mathrm{P}=0.020)$. These results point to an asymmetrical brainstem involvement in the neurodegenerative process, mainly affecting the symptomatic brainstem side, and subsequently traveling along with the affected fibre tracts (i.e. the affected brainstem side is ipsilateral to the patient's resting tremors, rigidity, and bradykinesia at the pontomedullary junction below the level of the vestibular nuclei; and the affected fibre tracts cross over to the contralateral brainstem side above the level of the vestibular nuclei along with the decussation of the medial longitudinal fasciculus). There is just one prior study by Pötter-Nerger et al. ${ }^{41}$, which only shows asymmetrical abnormalities of the ocular VEMP tests involving the symptomatic brainstem side, whereas the cervical VEMP tests did not show these asymmetrical abnormalities. However, the differences between Pötter-Nerger et al. ${ }^{41}$ 's study and ours can possibly be explained by the difference in population size (i.e. 30 PD patients in our study versus 13 patients in the study by Pötter-Nerger et al. ${ }^{41}$ ), as small differences between groups are more easily detected in a larger study population. Our study demonstrates that not only the central vestibular brainstem pathways are affected at the symptomatic brainstem side, but also that the central auditory brainstem pathways seem to be affected, which is illustrated by the prolonged III-V BAEP interlatencies at the symptomatic brainstem side. As discussed earlier, the main BAEP and VEMP abnormalities in our study consisted of severely prolonged cervical VEMP p13 latency, ocular VEMP n1 latency, and BAEP III-V inter-latency prolongations 
(i.e. p13 mean latency prolongation of $134 \%$ upper limit of normal (ULN); n1 mean latency prolongation of $128 \%$ ULN; mean III-V BAEP inter-latency prolongation of $120 \%$ ULN) with preserved amplitudes ${ }^{37}$. As discussed in paragraph 1.1 of this chapter, asymmetrically severely prolonged evoked potential latencies combined with relatively preserved amplitudes can theoretically be caused by a primary demyelinating neurodegeneration. However, the pathophysiological distinction between axonal and demyelinating central nervous system pathologies based upon evoked potential measurements is purely speculative and has to our knowledge not been studied earlier in literature. At this moment, clear neurophysiological criteria for this pathophysiological differentiation in central nervous system disorders are lacking, in contrast to the neurophysiological criteria that are present for this differentiation of the peripheral neuropathies. Further research concerning this pathological differentiation between axonal and demyelinating central nervous system disorders, based upon evoked potential measurements seems interesting, as this gives important insights into the pathophysiological hallmark of the underlying disease.

Parkinson's disease is defined pathophysiologically by the presence of abnormal alphasynuclein protein aggregations in neurons and glial cells, and by progressive neurodegeneration of selected brain regions (e.g. cholinergic pedunculopontine nucleus, dopaminergic substantia nigra and ventral tegmental area). These protein aggregates are called: a) Lewy bodies in neuronal perikarya, b) Lewy neuritis in neuronal processes, and c) coiled bodies in affected oligodendrocytes ${ }^{43,44}$. Also, multiple system atrophy (MSA) - one of the forms of atypical parkinsonism - is characterized by neuronal and glial alpha-synuclein aggregates, however, it is generally believed that neurons in MSA also have inclusions within their brainstem nuclei in contrast to $\mathrm{PD}^{44}$. Seidel et al. ${ }^{43}$ demonstrated Lewy bodies and Lewy neuritis in all cranial nerve nuclei, premotor oculomotor, precerebellar, and vestibular nuclei for the first time in PD patients; this contrasted with the general belief that such abnormalities were limited to MSA patients. Moreover, Lewy neuritis and coiled bodies were demonstrated in all brainstem fibre tracts. These abnormalities may be the result of a transneuronal disease spread along the anatomical pathways due to disturbed intra-axonal transport processes ${ }^{43}$. When we combine both the neuropathological and neurophysiological research data in PD patients, a transneuronal disease progression along the anatomical pathways, asymmetrically affecting the brainstem seems most likely (i.e. brainstem fibre tracts ipsilateral to the patient's clinically most affected side at the medullopontine junction, and contralateral to the patient's clinically most affected side above the motor decussation at the pontine and pontomesencephalic junction). However, asymmetrical brainstem degeneration spreading along with the brainstem fibre tracts below the level of the substantia nigra 
in the mesencephalon has not yet been confirmed in neuropathological studies and seems an interesting topic for future research. The neuropathological brainstem abnormalities suggest a neurodegenerative process with a primary axonal hallmark ${ }^{43}$, however the electrodiagnostic evoked potential abnormalities (i.e. severely delayed latencies with relatively spared amplitudes) suggest an important demyelinating neurodegenerative component. However, recent experimental evidence in different animal models suggests that synaptic dysfunction is an early and important feature in $\mathrm{PD}^{45}$, which might also (partially) explain the evoked potential abnormalities found in VEMP and BAEP testing (apart from the possible concomitant primary axonal and or demyelinating features of the disorder). Future neuropathological brainstem studies in PD should therefore also focus on the myelination of affected brainstem fibre tracts containing Lewy neuritis and coiled bodies, with the primary question whether concomitant demyelination is an important part of the neurodegenerative process. Obviously, more studies are needed concerning the process of synaptic transmission to confirm whether the synaptic dysfunction, which is found in animal $\mathrm{PD}$ models, is present in PD patients.

\section{Summary chapter 6}

The one-year follow-up results of the case-control study described above in paragraph 2 of this chapter, and which is discussed in chapter 5 in more detail, are discussed in Chapter 6. All 25 healthy volunteers, 14 AP, and 30 PD patients were contacted for a telephone interview one-year following the baseline examinations, and only 1 AP (MSA) patient was lost to follow-up (attrition bias of only $1.4 \%$ ). All participants were questioned about their fall frequency based upon their fall diaries, and their subjective balance confidence (according to the ABC-16 questionnaire). The results were compared to the baseline results. The primary aim was to determine whether neurovestibular laboratory tests (collected at baseline) can predict future falls in PD and AP patients. Cervical and ocular VEMPs combined with clinical tests for postural imbalance were the best predictors of future fall incidents, both in $\mathrm{PD}$ and AP groups, with a sensitivity of $100 \%$. The positive predictive value was $68.2 \%$ when only one VEMP test was abnormal, and $83.3 \%$ when both VEMP tests were abnormal. The fall frequency at baseline and after one year was significantly higher and the balance confidence scale (ABC-16) was significantly lower in both the PD and AP groups compared to healthy controls. Therefore, abnormal VEMP tests are associated with an increased risk of future fall incidents in $\mathrm{PD}$ and $\mathrm{AP}$ patients with postural imbalance in the absence of freezing of gait $^{46}$. 
A screening test is only useful in clinical practice when it has both a high sensitivity and a positive predictive value (PPV). Knowing that freezing of gait is a common risk factor for falls $s^{47,48}$, we examined the association between freezing at baseline and the subsequent risk of falls. In our study, assessment of freezing of gait (by qualitative assessment of straight walking in combination with the assessment of rapid clockwise and count-clockwise turning) was the clinical test with the highest PPV (namely $87.5 \%$ ), but the sensitivity was only $46.9 \%$ (as only 7 of the 15 falling patients were detected). This makes testing for freezing of gait by itself inadequate for screening purposes, since half of the potential fallers are missed. Moreover, clinical testing for postural imbalance (using the retropulsion test) had a sensitivity of $100 \%$ for detection of falling among PD and AP patients, but the PPV was only $46.9 \%$. So again, this clinical test is, by itself, unsuitable for screening purposes because about half of the patients with abnormal results will not fall in the following year. However, the combination of both cervical and ocular VEMP testing, combined with clinical testing for postural imbalance, seemed to be the optimal combination (yielding a sensitivity of $100 \%$ for detecting patients at risk of future falling, with a PPV of $68.2 \%$ for one abnormal VEMP test and a PPV of $83.3 \%$ when both VEMP tests are abnormal). However, the presence of freezing of gait is such a strong, but insensitive, predictor for future falls in both AP and PD patients that VEMP testing in these patients does not have any additional value (i.e. all patients with freezing of gait also had postural imbalance and abnormal VEMPs). Therefore, I concluded that cervical and ocular VEMP testing can possibly give additional information concerning the future fall risk in selected PD and AP patients (i.e. those patients with postural imbalance in the absence of freezing of gait $)^{46}$. However, these results, as discussed above, must be interpreted cautiously as the studied groups contained small numbers of patients, with a heterogeneous population in the AP patient group.

One can speculate whether this additional information will aid in guiding future fall prevention therapies as $\mathrm{PD}$ and AP patients with postural imbalance already have a high risk for falling. Multiple meta-analyses and Cochrane reviews of physiotherapy in PD reported short-term statistically significant, but clinically modest positive effects concerning balance-related activity performance and improvement of gait. However, there was no significant evidence concerning the reduction of the risk of falling ${ }^{49-52}$. Moreover, one Cochrane review ${ }^{49}$ compared the different physical therapies and concluded that a formal qualitative comparison was not possible because of the heterogeneity of the studied techniques, small sample sizes of the studied groups, methodological flaws, and risk of publication bias. Therefore, there is no evidence to choose or advise one form of physical therapy over another, as was concluded by the authors $^{49}$. High-quality prospective studies with large study groups and long-term 
follow-up are needed to assess the effect of physiotherapy in PD and AP concerning the reduction of the risk of falling, and to assess which form of physical therapy is superior in achieving this goal. Such studies should follow evidence-based guidelines for physiotherapy strategies in $\mathrm{PD}^{53,54}$, and the interventions should ideally be delivered by therapists experienced in the management of patients with $\mathrm{PD}^{55,56}$.

A recent single-case study described the motor and non-motor effects of repeated caloric vestibular stimulation in a patient with $\mathrm{PD}^{57}$. The motor performance and the non-motor scores (i.e. Montreal cognitive assessment, hospital depression scale, and Epworth sleepiness scale) improved significantly during repeated caloric vestibular stimulation and persisted during follow-up 5 months after stimulation. Moreover, these improvements were not seen during the first phase of the study which consisted of sham-stimulation. Moreover, recent studies in PD patients provide preliminary evidence that (stochastic) galvanic vestibular stimulation can possibly significantly reduce postural instability, improve corrective postural responses, improve anterior bending posture, and is, above all, safe for short term use ${ }^{58-62}$. These studies, however, consist of single case-reports or small case series without further evidence, and should therefore in my opinion be considered as proof-of-concept evidence. Obviously, large scale randomized controlled double-blinded trials are needed in PD and AP patients to demonstrate whether (stochastic) galvanic vestibular or repeated caloric stimulation is able to clinically and statistically significantly reduce fall episodes and/or to improve motor and non-motor symptoms.

At this moment, I do not advise to perform VEMP testing in PD or AP patients as it does not have any treatment consequences. However, vestibular laboratory testing (i.e. VEMP, SVV, and or videonystagmography with caloric and rotatory chair stimulation) might be helpful in elucidating the underlying physiological changes secondary to repeated vestibular stimulation as mentioned earlier.

\section{Highlights of the most important findings}

- Abnormal VEMP and SVV laboratory test results can give important clues to the localization of the underlying neurovestibular problem, but are not disease specific.

- Approximately $90 \%$ of PD patients and $100 \%$ of AP patients have abnormal neurovestibular laboratory test results, with a predominantly central neurovestibular dysfunction profile.

- In PD patients, it is mainly the symptomatic brainstem side that is electrophysiologically affected, compared to healthy volunteers (i.e. the affected brainstem side is ipsilateral to the patient's resting tremors, rigidity, and bradykinesia at the pontomedullary junction below the level of the vestibular nuclei; and the 
affected fibre tracts cross over to the contralateral brainstem side above the level of the vestibular nuclei along with the decussation of the medial longitudinal fasciculus)

- PD and AP patients with laboratory signs of neurovestibular dysfunction have a statistically significant increased risk of falling.

- Cervical and ocular VEMP testing in combination with clinical testing for postural imbalance can predict future fall incidents in PD and AP patients (sensitivity of $100 \%$, PPV of $68.2 \%$ with one abnormal VEMP test, and a PV of $83.3 \%$ when both VEMP tests are abnormal). 


\section{References}

1. Curthoys, I. S. A critical review of the neurophysiological evidence underlying clinical vestibular testing using sound, vibration and galvanic stimuli. Clin. Neurophysiol. 121, 132-144 (2010).

2. Eleftheriadou, A. \& Koudounarakis, E. Vestibular-evoked myogenic potentials eliciting: An overview. European Archives of Oto-Rhino-Laryngology 268, 331-339 (2011).

3. Rosengren, S. M., Welgampola, M. S. \& Colebatch, J. G. Vestibular evoked myogenic potentials: Past, present and future. Clin. Neurophysiol. 121, 636-651 (2010).

4. Rosengren, S. \& Kingma, H. New perspectives on vestibular evoked myogenic potentials. Curr. Opin. Neurol. 26, 74-80 (2013).

5. Venhovens, J., Meulstee, J. \& Verhagen, W. Vestibular Evoked Myogenic Potentials (VEMPs) in Central Neurological Disorders. Clin. Neurophysiol. 127, 40-49 (2016).

6. Colebatch, J. G. Sound conclusions? Clin. Neurophysiol. 121, 124-126 (2010).

7. Curthoys, I. S. A balanced view of the evidence leads to sound conclusions. A reply to J.G. Colebatch 'Sound conclusions?'. Clin. Neurophysiol. 121, 977-8 (2010).

8. Papathanasiou, E. S. Ocular vestibular evoked myogenic potentials (OVEMPs): saccule or utricle? Clin. Neurophysiol. 123, 216 (2012).

9. Papathanasiou, E. S. \& Papacostas, S. S. Vestibular evoked myogenic potentials: the fuzzy picture of different stimulation types is beginning to come into focus. Clin. Neurophysiol. 124, 1926-7 (2013).

10. Todd, N. The ocular vestibular evoked myogenic potential (OVEMP), ten years old. Clin. Neurophysiol. 125, 439-41 (2014).

11. Venhovens, J., Meulstee, J. \& Verhagen, W. I. M. Ocular and Cervical Vestibular Evoked Myogenic Potentials (VEMPs) in healthy volunteers: the intra-, interobserver, and the test re-test reliability. J. Vestib. Res. 25, 161-7 (2015).

12. Brantberg, K. Vestibular evoked myogenic potentials (VEMPs): Usefulness in clinical neurotology. Seminars in Neurology 29, 541-547 (2009).

13. Yew, A. et al. Characteristics and management of superior semicircular canal dehiscence. J. Neurol. Surg. B. Skull Base 73, 365-70 (2012).

14. Zuniga, M. \& Janky, K. Ocular vs. cervical VEMPs in the diagnosis of superior semicircular canal dehiscence syndrome. Otol. Neurotol. Off. Publ. Am. Otol. Soc. Am. Neurotol. Soc. [and] Eur. Acad. Otol. Neurotol. Neurotol. 34, 121-126 (2013).

15. Tankisi, H., Pugdahl, K., Johnsen, B. \& Fuglsang-Frederiksen, A. Correlations of nerve conduction measures in axonal and demyelinating polyneuropathies. Clin. Neurophysiol. 118, 2383-2392 (2007).

16. Chung, T., Prasad, K. \& Lloyd, T. E. Peripheral neuropathy. Clinical and electrophysiological considerations. Neuroimaging Clinics of North America 24, 49-65 (2014).

17. Nuwer, M. R. Fundamentals of evoked potentials and common clinical applications today. Electroencephalography and Clinical Neurophysiology 106, 142-148 (1998).

18. Lascano, A. M., Lalive, P. H., Hardmeier, M., Fuhr, P. \& Seeck, M. Clinical evoked potentials in neurology: a review of techniques and indications. J. Neurol. Neurosurg. Psychiatry jnnp-2016314791 (2017). doi:10.1136/jnnp-2016-314791

19. O.N., M. Brainstem auditory evoked potentials. J. Clin. Neurophysiol. 11, 319-342 (1994).

20. Vanspauwen, R., Wuyts, F. L. \& Van De Heyning, P. H. Validity of a new feedback method for the VEMP test. Acta Otolaryngol. 126, 796-800 (2006).

21. Vanspauwen, R., Wuyts, F. L. \& Van De Heyning, P. H. Vestibular evoked myogenic potentials: Test-retest reliability and normative values obtained with a feedback method for the sternocleidomastoid muscle contraction. J. Vestib. Res. Equilib. Orientat. 19, 127-135 (2009).

22. Nguyen, K. D., Welgampola, M. S. \& Carey, J. P. Test-retest reliability and age-related characteristics of the ocular and cervical vestibular evoked myogenic potential tests. Otol. Neurotol. 31, 793-802 (2010).

23. Venhovens, J., Meulstee, J. \& Verhagen, W. I. M. Static Subjective Visual Vertical in Healthy Volunteers: The Effects of Different Preset Angle Deviations and Test-Retest Variability. Neuroophthalmology. 40, 113-119 (2016). 
24. Baccini, M., Paci, M., Del Colletto, M., Ravenni, M. \& Baldassi, S. The assessment of subjective visual vertical: comparison of two psychophysical paradigms and age-related performance. Atten. Percept. Psychophys. 76, 112-22 (2014).

25. Brandt, T. \& Dieterich, M. Vestibular syndromes in the roll plane: topographic diagnosis from brainstem to cortex. Ann. Neurol. 36, 337-47 (1994).

26. Tarnutzer, a a, Bockisch, C., Straumann, D. \& Olasagasti, I. Gravity dependence of subjective visual vertical variability. J. Neurophysiol. 102, 1657-1671 (2009).

27. Yelnik, A. P. et al. Perception of verticality after recent cerebral hemispheric stroke. Stroke 33, 2247-2253 (2002).

28. Dieterich, M. \& Brandt, T. Ocular torsion and tilt of subjective visual vertical are sensitive brainstem signs. Ann. Neurol. 33, 292-299 (1993).

29. Dieterich, M. \& Brandt, T. Ocular torsion and perceived vertical in oculomotor, trochlear and abducens nerve palsies. Brain 116, 1095-1104 (1993).

30. Brandt, T., Dieterich, M. \& Danek, A. Vestibular cortex lesions affect the perception of verticality. Ann. Neurol. 35, 403-412 (1994).

31. Pagarkar, W., Bamiou, D.-E., Ridout, D. \& Luxon, L. M. Subjective visual vertical and horizontal: effect of the preset angle. Arch. Otolaryngol. Head. Neck Surg. 134, 394-401 (2008).

32. Zwergal, A. et al. A bucket of static vestibular function. Neurology 72, 1689-1692 (2009).

33. Brodsky, J. R., Cusick, B. A., Kawai, K., Kenna, M. \& Zhou, G. Peripheral vestibular loss detected in pediatric patients using a smartphone-based test of the subjective visual vertical. Int. J. Pediatr. Otorhinolaryngol. 79, 2094-2098 (2015).

34. Wood, B. H., Bilclough, J. A., Bowron, A. \& Walker, R. W. Incidence and prediction of falls in Parkinson's disease: a prospective multidisciplinary study. J. Neurol. Neurosurg. Psychiatry 72, $721-$ 725 (2002).

35. Bloem, B. \& Bhatia, K. in Clinical disorders of balance, posture and gait. (ed. Bronstein AM, Brandt T, Nutt JG, W. M.) 173-206 (Arnold: London, 2004).

36. Grimbergen, Y. A. M., Munneke, M. \& Bloem, B. R. Falls in Parkinson's disease. Curr. Opin. Neurol. 17, 405-415 (2004).

37. Venhovens, J., Meulstee, J., Bloem, B. R. \& Verhagen, W. I. M. Neurovestibular analysis and falls in Parkinson's disease and atypical parkinsonism. Eur. J. Neurosci. 43, 1636-46 (2016).

38. Critchley, R. J., Khan, S. K., Yarnall, A. J., Parker, M. J. \& Deehan, D. J. Occurrence, management and outcomes of hip fractures in patients with Parkinson's disease. Br. Med. Bull. 115, 135-42 (2015).

39. van der Marck, M. A. et al. Consensus-based clinical practice recommendations for the examination and management of falls in patients with Parkinson's disease. Parkinsonism and Related Disorders 20, 360-369 (2014).

40. Thijs, R. D., Bloem, B. R. \& Dijk, J. G. Falls, faints, fits and funny turns. J. Neurol. 256, 155-167 (2009).

41. Pötter-Nerger, M., Govender, S., Deuschl, G., Volkmann, J. \& Colebatch, J. G. Selective changes of ocular vestibular myogenic potentials in Parkinson's disease. Mov. Disord. 30, 584-9 (2015).

42. Pötter-Nerger, M., Reich, M. M., Colebatch, J. G., Deuschl, G. \& Volkmann, J. Differential effect of dopa and subthalamic stimulation on vestibular activity in Parkinson's disease. Mov. Disord. 27, 1268-75 (2012).

43. Seidel, K. et al. The brainstem pathologies of Parkinson's disease and dementia with Lewy bodies. Brain Pathol. 25, 121-35 (2015).

44. Dickson, D. W. Parkinson's disease and parkinsonism: neuropathology. Cold Spring Harb. Perspect. Med. 2, (2012).

45. Schirinzi, T. et al. Early synaptic dysfunction in Parkinson's disease: Insights from animal models. Mov. Disord. 0, 1-12 (2016).

46. Venhovens, J., Meulstee, J., Bloem, B. R. \& Verhagen, W. I. M. Neurovestibular dysfunction and falls in Parkinson's disease and atypical Parkinsonism: a prospective one year follow-up study. Artic. Submitt.

47. Bloem, B. R., Hausdorff, J. M., Visser, J. E. \& Giladi, N. Falls and freezing of gait in Parkinson's disease: A review of two interconnected, episodic phenomena. Mov. Disord. 19, 871-884 (2004).

48. Okuma, Y. Freezing of gait and falls in Parkinson's disease. J. Parkinsons. Dis. 4, 255-60 (2014). 
49. Tomlinson, C. L. et al. Physiotherapy for Parkinson's disease: a comparison of techniques. Cochrane database Syst. Rev. CD002815 (2014). doi:10.1002/14651858.CD002815.pub2

50. Tomlinson, C. L. et al. Physiotherapy versus placebo or no intervention in Parkinson's disease. Cochrane database Syst. Rev. CD002817 (2013). doi:10.1002/14651858.CD002817.pub4

51. Tomlinson, C. et al. Physiotherapy intervention in Parkinson's disease: systematic review and metaanalysis. Bmj 345, e5004 (2012).

52. Allen, N. E., Sherrington, C., Paul, S. S. \& Canning, C. G. Balance and falls in Parkinson's disease: a meta-analysis of the effect of exercise and motor training. Mov. Disord. 26, 1605-15 (2011).

53. Keus, S. H. J., Munneke, M., Nijkrake, M. J., Kwakkel, G. \& Bloem, B. R. Physical therapy in Parkinson's disease: evolution and future challenges. Mov. Disord. 24, 1-14 (2009).

54. Radder, D. L. M. et al. Physical therapy and occupational therapy in Parkinson's disease. Int. J. Neurosci. 1-14 (2017). doi:10.1080/00207454.2016.1275617

55. Bloem, B. R. \& Munneke, M. Revolutionising management of chronic disease: the ParkinsonNet approach. BMJ 348, g1838-g1838 (2014).

56. de Vries, N. M., Sturkenboom, I. H. \& Bloem, B. R. Physiotherapy and Occupational Therapy and Mild to Moderate Parkinson Disease. JAMA Neurol. 73, 893-4 (2016).

57. Wilkinson, D., Podlewska, A. \& Sakel, M. A durable gain in motor and non-motor symptoms of Parkinson's Disease following repeated caloric vestibular stimulation: A single-case study. NeuroRehabilitation 38, 179-82 (2016).

58. Samoudi, G., Jivegård, M., Mulavara, A. P. \& Bergquist, F. Effects of Stochastic Vestibular Galvanic Stimulation and LDOPA on Balance and Motor Symptoms in Patients With Parkinson's Disease. Brain Stimul. 8, 474-80

59. Rizzo-Sierra, C. V, Gonzalez-Castaño, A. \& Leon-Sarmiento, F. E. Galvanic vestibular stimulation: a novel modulatory countermeasure for vestibular-associated movement disorders. Arq. Neuropsiquiatr. 72, 72-7 (2014).

60. Kataoka, H. et al. Can Postural Instability Respond to Galvanic Vestibular Stimulation in Patients with Parkinson's Disease? J. Mov. Disord. 9, 40-3 (2016).

61. Okada, Y. et al. Galvanic vestibular stimulation may improve anterior bending posture in Parkinson's disease. Neuroreport 26, 405-10 (2015).

62. Pal, S., Rosengren, S. M. \& Colebatch, J. G. Stochastic galvanic vestibular stimulation produces a small reduction in sway in Parkinson's disease. J. Vestib. Res. 19, 137-42 (2009). 


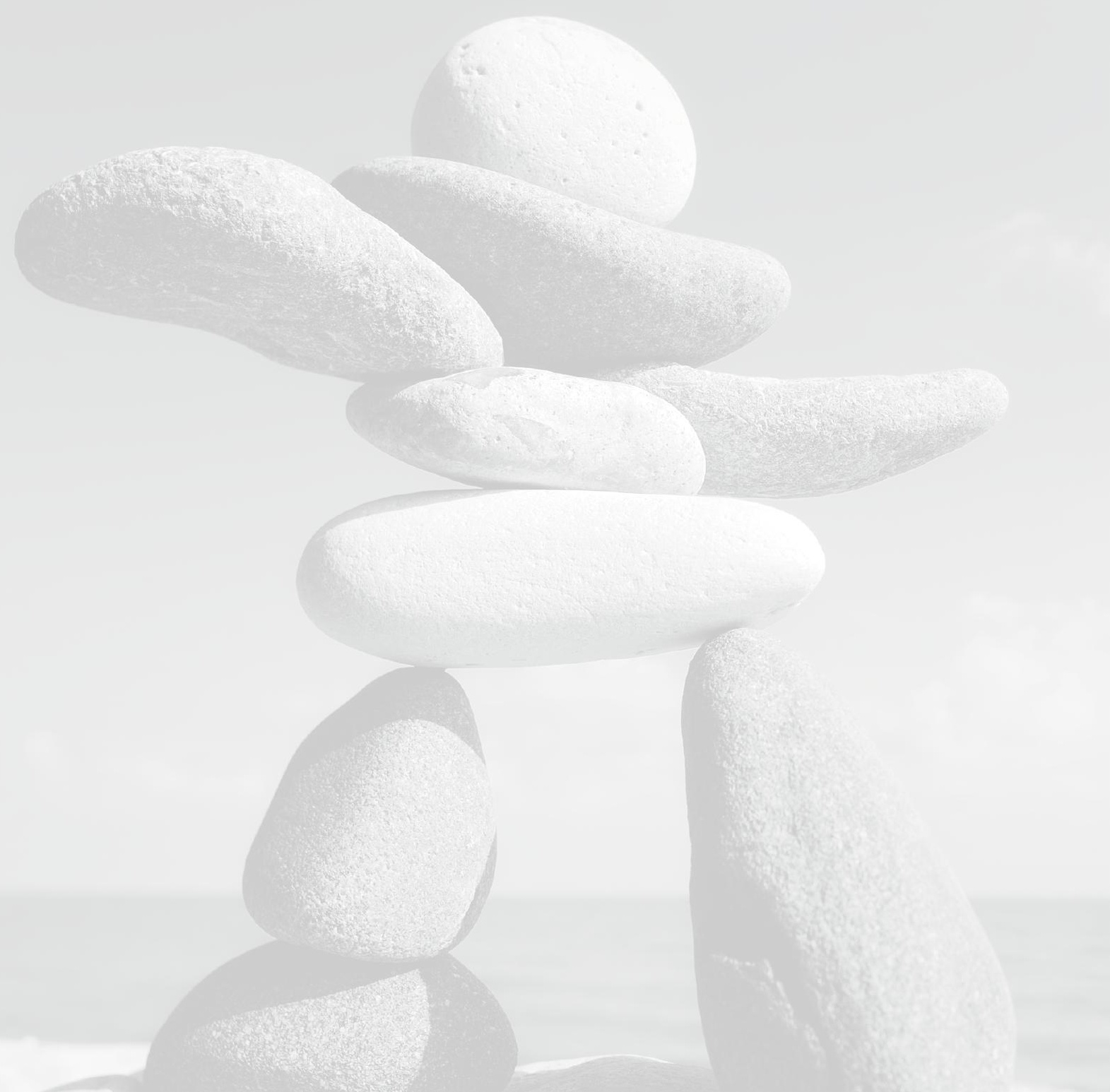




\section{Chapter 8}

Nederlandse samenvatting, algemene discussie en perspectieven voor toekomstig wetenschappelijk onderzoek 
Deze thesis is verdeeld in twee delen welke apart van elkaar zullen worden bediscussieerd. Het eerste deel omvat technische en praktische problemen van neurovestibulaire onderzoeken met de nadruk op vestibulaire geëvoceerde potentialen (VEMP) en de statische subjectieve visuele verticaliteit (SVV). Het tweede deel geeft een gedetailleerd overzicht van een wetenschappelijke studie met betrekking tot neurovestibulaire onderzoeken bij personen met de ziekte van Parkinson (PD) en atypische parkinsonisme (AP) - zowel de baseline resultaten als na een follow-up duur van één jaar. Ieder deel begint met een korte samenvatting van het betreffende hoofdstuk en wordt gevolgd een algemene discussie en perspectieven voor toekomstig wetenschappelijk onderzoek.

\section{DEEL EEN}

\section{Neurovestibulaire testen - praktische en technische problemen}

Vestibulair geëvoceerde potentialen (VEMP)

\section{Samenvatting hoofdstukken 2 en 3}

Vestibulair geëvoceerde potentialen testen (VEMP) kwamen ongeveer twee decades geleden in gebruik als testen welke geschikt zijn voor gedetailleerde neurofysiologische beoordeling van het centrale en perifere vestibulaire systeem. VEMP testen kunnen worden onderverdeeld in een cervicale en oculaire respons. De cervicale VEMP test beoordeelt de integriteit van de vestibulospinale reflexboog welke begint bij de sacculus om uiteindelijk een inhibitoir motore respons te veroorzaken in de ipsilaterale sternocleidomastoïdeus spier (door vervolgens via de ipsilaterale inferieure vestibulaire zenuw, vestibulaire kernen, mediale vestibulospinale tractus, cervicale spinale motore neuronen en de nervus accessorius te reizen) $)^{1-5}$. De oculaire VEMP test beoordeelt de integriteit van de vestibulo-oculaire reflexboog welke meest waarschijnlijk begint in de utriculus (over de precieze origine wordt echter uitgebreid gedebatteerd in de literatuur en met name of de exacte origine voornamelijk de utriculus is of een combinatie van de utriculus en de sacculus) om uiteindelijk een excitatoire motore respons te veroorzaken in de contralaterale musculus obliquus inferior (door vervolgens via de ipsilaterale superieure vestibulaire zenuw, vestibulaire kernen, fasciculus longitudinalis medialis, contralaterale nucleus oculomotorius en nervus oculomotorius te reizen $)^{1-10}$. Voor een schematische representatie van deze 
complexe neurofysiologische baansystemen ${ }^{5}$ van de vestibulo-oculaire en vestibulospinale reflexbogen verwijzen we gaarne naar figuur 1, hoofdstuk 2. Voor een voorbeeld met betrekking tot de normale cervicale en oculaire VEMP responsen verwijzen we u gaarne naar figuur 2, hoofdstuk 2 . Hoofdstuk 2 geeft een gedetailleerd overzicht van de VEMP afwijkingen in verschillende centraal neurologische aandoeningen, de interpretatie van deze VEMP responsen en mogelijke klinische toepassingen. Hiervoor werd de beschikbare wetenschappelijke literatuur kritisch beoordeeld en vervolgens werd op basis van deze gegevens het niveau van diagnostische evidentie ingeschat. Het algemene niveau van diagnostische evidentie volgens het Oxford centrum voor evidence-based medicine bestaat voornamelijk uit een graad 4 evidentie voor het gebruik van VEMP testen voor centraal neurologische aandoeningen met enkele uitzonderingen welke een maximum graad van $2 \mathrm{~b}$ bereiken (voor Multiple Sclerosis, vestibulaire schwannomen, ziekte van Parkinson en herseninfarcten in het vertebrobasilaire stroomgebied). Hierbij blijft de totale diagnostische evidentie met betrekking tot VEMP afwijkingen in centraal neurologische aandoeningen erg beperkt op het moment. VEMP afwijkingen worden regelmatig gezien in centraal neurologische aandoeningen en gedetailleerde interpretatie van de resultaten kan belangrijke lokaliserende informatie geven, echter de afwijkingen zijn meestal wel aspecifiek met betrekking tot de onderliggende ziekte. Voor een gedetailleerde beschrijving van de te verwachten VEMP afwijkingen voor aandoeningen op verschillende anatomische lokalisaties verwijzen we gaarne naar tabel 1 , hoofdstuk 2 . In hoofdstuk 3 wordt een prospectieve oculaire en cervicale VEMP studie beschreven met betrekking tot de intra-, interbeoordelaar en test her-test betrouwbaarheid in twintig gezonde studie deelnemers. De intra- en interbeoordelaar betrouwbaarheid, welke een belangrijk aspect is van klinische VEMP beoordeling, betreffende de drie ervaren klinische neurofysiologen is zeer goed voor zowel de oculaire als cervicale VEMP responsen. De groepsresultaten met betrekking tot de test en de her-test waren vergelijkbaar en er was geen significant verschil tussen de groepen. De individuele resultaten, echter vertoonden wel een relevante variabiliteit waarbij alle resultaten wel binnen de normale grenzen bleven. De oculaire en cervicale VEMP amplitudes waren de meest betrouwbare test her-test parameters gevolgd door de cervicale VEMP latenties. De oculaire VEMP latenties vertoonden een matige test her-test betrouwbaarheid ${ }^{11}$.

VEMP afwijkingen worden regelmatig gezien bij patiënten met zowel centrale als perifere neurovestibulaire aandoeningen en overige centraal neurologische 
aandoeningen ${ }^{5,12}$. Gedetailleerde interpretatie van deze afwijkingen kan mogelijks belangrijke lokaliserende informatie geven met betrekking tot de zijde en origine van de laesie binnen het centrale en perifere vestibulaire systeem (wat de voornaamste kracht van VEMP testen is). De uitslagen van VEMP testen bij verdenking op een perifeer vestibulaire problematiek moeten echter met grote zorg worden geïnterpreteerd wanneer er bij een patiënt sprake is van een centraal neurologische aandoening en vice versa. Hoofdstuk 2 geeft aanvullende informatie met betrekking tot de mogelijke anatomische lokalisaties van VEMP afwijkingen. De diagnostische graad van evidencebased medicine voor het gebruik van VEMP testen voor centraal neurologische aandoeningen is momenteel erg beperkt tot een algemene graad 4 evidentie met enkele uitzonderingen welke een maximum graad van $2 \mathrm{~b}$ bereiken (voor Multiple Sclerosis, vestibulaire schwannomen, ziekte van Parkinson en herseninfarcten in het vertebrobasilaire stroomgebied). De primaire indicatie voor het uitvoeren van VEMP testen is daardoor in mijn opinie enkel patiënten wier neurovestibulaire klachten niet betrouwbaar kunnen worden gelokaliseerd in het centrale of perifere vestibulaire systeem noch met MRI-cerebrum, noch met videonystagmografie met calorisatie ${ }^{5}$. Een uitzondering is de diagnostische work-up van een patiënt met een verdenking op een superieure semicirculair kanaal dehiscentie syndroom (SCDS) waarbij er ipsilateraal verminderde cervicale VEMP stimulatie drempels zijn en voornamelijk toegenomen contralaterale oculaire VEMP amplitudes. Deze VEMP afwijkingen zijn een sensitief teken bij het lokaliseren van de aangedane zijde. De voornaamste oorzaak van dit fenomeen is waarschijnlijk een toegenomen geluid en drukgevoeligheid van het ipsilaterale labyrint in vergelijking met de contralaterale zijde $e^{13,14}$.

VEMP afwijkingen zijn in het algemeen niet ziekte specifiek en geven daardoor geen additionele informatie met betrekking tot de etiologie van de onderliggende ziekte. Men kan er echter over speculeren of gedetailleerde interpretatie van de VEMP afwijkingen geen inzicht kan geven in de onderliggende pathofysiologische kenmerken van de ziekte (of de onderliggende ziekte primair demyeliniserend, axonaal of een combinatie van beiden is) vergelijkbaar met bijvoorbeeld perifere neuropathieën. Demyeliniserende perifere neuropathieën kenmerken zich door een forse reductie van de zenuwgeleidingssnelheden, conductie blokkades, toegenomen temporale dispersie, fors vertraagde F-waves en/of fors vertraagde distale zenuwgeleidingstijden. Daarentegen worden axonale perifere neuropathieën gekenmerkt door afgenomen amplitudes in de anwezigheid van relatief gespaarde zenuwgeleidingssnelheden ${ }^{15,16}$.

Op dit moment zijn er geen betrouwbare neurofysiologische criteria voor geëvoceerde potentialen welke de differentiatie tussen primair demyeliniserende en axonale 
aandoeningen in het centrale zenuwstelsel mogelijk maken. Neuritis optica in Multiple Sclerosis bijvoorbeeld (welke zich pathofysiologisch kenmerkt door primaire demyelinisatie) veroorzaakt typisch asymmetrisch sterk verlengde latenties van de ipsilaterale visueel geëvoceerde potentialen meting (VEP) met relatief gespaarde amplitudes. Opticus neuropathieën ten gevolge van externe compressie, ischemie, metabole ontregelingen en neurodegeneratieve aandoeningen echter veroorzaken meestal minder uitgesproken latentie verlengingen en een meer uitgesproken reductie van de VEP amplitude ${ }^{17,18}$. Afwijkingen met betrekking tot de auditieve hersenstam geëvoceerde potentialen (BAEP) in Multiple Sclerosis patiënten zijn daarentegen erg aspecifiek en veroorzaken voornamelijk top IV en $\mathrm{V}$ afwijkingen gevolgd door verlenging van de intertop I-V en voornamelijk III-V latenties. De bovenbeschreven BAEP afwijkingen zijn echter niet typisch voor demyeliniserende aandoeningen en worden ook gevonden in vele andere centraal neurologische aandoeningen zoals hersenstam gliomen, spinocerebellaire degeneratieve aandoeningen en hydrocephalus ${ }^{19}$. De VEMP resultaten in Multiple Sclerosis patiënten tonen voornamelijk toegenomen latenties gevolgd door afwezige responsen. Toegenomen latenties worden echter ook beschreven in patiënten met de ziekte van Alzheimer, migraine en met een vertebrobasilair herseninfarct (bij de laatste aandoening hadden de meeste patiënten afgenomen amplitudes) $)^{5}$.

Concluderend kan men op hypothetische en puur speculatieve basis beargumenteren dat sterk asymmetrisch toegenomen VEMP latenties (meer dan 130 procent van de bovenste limiet van de normaalwaarden) met relatief gespaarde amplitudes en golf morfologie mogelijks worden veroorzaakt door demyeliniserende aandoeningen (vergelijkbaar met de VEP afwijkingen in neuritis optica en zenuwgeleidingsbevindingen in perifere neuropathieën). Omgekeerd kan eveneens worden beweerd dat geëvoceerde potentialen metingen op dit moment (en voornamelijk de BAEP en VEMP testen) geen pathofysiologische differentiatie toelaten met betrekking tot de onderliggende aandoening. Dit is gebaseerd op de BAEP en VEMP case-beschrijvingen in de literatuur bij verschillende aandoeningen welke geen primair demyeliniserend kenmerk hebben (zoals vertebrobasilaire herseninfarcten en migraine). De bovenvermelde casebeschrijvingen in de literatuur bevatten helaas geen gedetailleerde beschrijvingen met betrekking tot de mate van latentietijd toename van de geëvoceerde potentialen waarbij de verwachting is dat deze veel meer uitgesproken zijn bij demyeliniserende aandoeningen in vergelijking met axonale aandoeningen. Verdere verduidelijking van deze pathofysiologische differentiatie voor centraal neurologische aandoeningen gebaseerd op geëvoceerde potentialen metingen lijkt een interessant onderzoeksgebied voor toekomstig onderzoek. Deze pathofysiologische differentiatie lijkt niet enkel nuttig voor diagnostische doeleinden, eveneens kan deze differentiatie behulpzaam zijn voor 
het ontrafelen van de onderliggende pathofysiologische mechanismen in verschillende centraal neurologische aandoeningen welke tot op heden niet volledig bekend zijn (zoals bijvoorbeeld verschillende neurodegeneratieve aandoeningen) en om hun progressie over het beloop van de tijd te volgen. Eenzelfde studie opzet kan hiervoor worden toegepast als werd gebruikt door de auteurs Tankisi et al. ${ }^{15}$ in hun studie naar perifere polyneuropathieën waarbij de geleidingssnelheden en amplitudes werden bepaald in aandoeningen met goed bekende pathofysiologische kenmerken, echter dan toegepast voor VEMP en BAEP metingen in centraal neurologische aandoeningen.

In hoofdstuk 3 wordt de intra- en interbeoordelaar betrouwbaarheid van VEMP beoordeling besproken welke zeer goed was in onze studie ${ }^{11}$. De studie toont eveneens aan dat ervaren klinisch neurofysiologen en neurologen met beperkte ervaring in de beoordeling van individuele VEMP curven in een korte tijd (ongeveer 45 minuten) kunnen worden getraind om deze beoordelingen betrouwbaar uit te voeren. De VEMP test her-test studie toont aan dat er een individuele variabiliteit is in de resultaten, echter dat deze allen binnen de normale grenzen blijven waarbij deze variabiliteit niet relevant lijkt te zijn voor gezonde proefpersonen. Men kan hier echter over speculeren of dit ook het geval is voor patiënten met abnormale test resultaten en of deze ook afwijkend blijven indien patiënten opnieuw getest worden. De oculaire en met name de cervicale VEMP amplitudes lijken de meest betrouwbare test her-test parameters te hebben, gevolgd door de cervicale VEMP latenties. De oculaire VEMP latenties vertonen echter een matige test her-test betrouwbaarheid. Standaardisatie van de cervicale VEMP amplitudes door de mate van sternocleidomastoideus spiercontractie te controleren gedurende de VEMP meting is eveneens een belangrijk aspect van het uitvoeren van VEMP metingen (bijvoorbeeld door een bloeddruk cuff en manometer te gebruiken waarbij een vooraf gekozen spierkracht kan worden aangehouden en gecontroleerd gedurende de meting zoals omschreven door de auteurs Vanspauwen et al. ${ }^{20,2 I}$ ). Een alternatieve methode hiervoor is om de cervicale VEMP amplitudes te corrigeren door het ruwe pre-stimulus EMG achtergrond signaal te gebruiken. Op dit moment zijn er helaas geen goed omschreven en gestandaardiseerde methoden om de oculaire VEMP amplitudes te corrigeren. Nguyen et al..$^{22}$ beschreven in hun studies een interessante methode om de oculaire VEMP amplitudes te standaardiseren welke tot onze kennis tot op heden nog niet uitvoerig bestudeerd is in de literatuur. De auteurs gebruikten verticale saccades voorafgaande de meting om de plaatsing van de elektroden te corrigeren. Indien het verschil tussen de amplitudes van de saccades tussen de beide ogen meer dan 25 procent bedroeg werden de elektrodes qua plaatsing gecorrigeerd. De oculaire VEMP test her-test studie van Nguyen et al. ${ }^{22}$ vertoonden een betere betrouwbaarheid (namelijk een zeer goede betrouwbaarheid) van de oculaire VEMP amplitudes in vergelijking met onze studie welke slechts matig tot goed was. Helaas verbeterde de algemeen matige betrouwbaarheid van 
de oculaire VEMP latenties niet met de bovenvermelde standaardisatie procedures. De bovenvermelde standaardisatie procedures voor de oculaire VEMP amplitudes lijken om de bovenstaande redenen effectief te zijn en daarom zeker de moeite waard om door middel van verder wetenschappelijk onderzoek verder uit te zoeken.

\section{Statische subjectieve visuele verticaliteit (SVV)}

\section{Samenvatting hoofdstuk 4}

Graviceptie is de mogelijkheid van een persoon om de oriëntatie relatief tot de zwaartekracht in te schatten welke kan worden gemeten door de (statische) subjectieve visuele verticaliteit (SVV). De graviceptieve baansystemen zorgen voor een multimodale sensore integratie van de vestibulaire, proprioceptieve en visuele input en bepaald aan de hand hiervan de relatieve positie van het lichaam ten opzichte van de absolute verticaliteit (zijnde het zwaartekrachtsveld van de aarde ${ }^{23-27}$ ). De resultaten van de statische SVV worden voornamelijk bepaald door de otoliet functie en balans waarbij dit de voornaamste receptoren in het perifere vestibulaire systeem zijn die gevoelig zijn voor de zwaartekracht (zijnde lineaire versnellingen). Neurovestibulaire laesies meer proximaal gelokaliseerd in zowel het perifere en centrale vestibulaire systeem kunnen natuurlijk ook afwijkingen in de statische SVV resultaten veroorzaken door verstoring van de graviceptieve baansystemen welke secundair resulteren in een otoliet dysbalans. Bijvoorbeeld statische SVV afwijkingen zijn een sensitief teken van hersenstam dysfunctie ${ }^{25,28}$. Voor het uitvoeren van de statische SVV testen verwijzen we naar hoofdstuk 4, methodologie paragraaf. In hoofdstuk $\mathbf{4}$ wordt een studie beschreven met 44 gezonde proefpersonen met betrekking tot de effecten van verschillende uitgangshoek deviaties en zijden en de test her-test variabiliteit. De statische SVV resultaten worden beïnvloed door de zijde van de uitgangshoek (Wilcoxon test, $\mathrm{P} \leq 0.001$ ), echter niet door de grote van de hoek zelf (uitgangshoeken van 10, 20 en 30 graden werden vergeleken). De test her-test resultaten van de gezonde proefpersonen waren vergelijkbaar op groepsniveau, echter vertoonden een relevante variabiliteit op individueel niveau met matige betrouwbaarheid. Alle individuele resultaten bleven echter binnen de normale grenzen ondanks deze variabiliteit. Het laatste doel van de studie was om een robuust statisch SVV protocol te exploreren en verder uit te bouwen waarbij zowel monoculaire als binoculaire metingen werden gebruikt. Het statische SVV studie protocol staat uitvoerig beschreven in hoofdstuk 4 . 
Het laatste doel van de studie in hoofdstuk $\mathbf{4}$ was om een praktisch statisch SVV protocol te exploreren en uit te bouwen met hierin zowel monoculaire als binoculaire metingen. Op dit moment is het vergelijken van literatuurbevindingen omtrent de statische SVV erg moeizaam gezien het ontbreken van een eenduidig test protocol waarbij veel auteurs de testen op verschillende manieren uitvoeren. Tevens worden op dit moment in de wetenschappelijke literatuur bijna alleen maar binoculaire metingen uitgevoerd, echter de toevoeging van monoculaire metingen gecombineerd met de binoculaire geeft additionele informatie met betrekking tot de mogelijke onderliggende lokalisatie van de laesie. Dieterich en Brandt $t^{25,28-30}$ toonden aan dat $94 \%$ van de 111 patiënten met acute hersenstam herseninfarcten een kant specifieke pathologische binoculaire statische SVV afwijking heeft. De binoculaire statische SVV afwijking is ipsiversief in perifere unilaterale vestibulaire aandoeningen en pontomedullaire laesies (onder het niveau waar de graviceptieve baansystemen kruisen naar de contralaterale zijde). De binoculaire statische SVV afwijkingen zijn contraversief in pontomesencephale aandoeningen en geeft betrokkenheid van de fasciculus longitudinalis medialis (FLM), interstitiële nucleus van Cajal (INC) en/of rostrale interstitiële nucleus van de FLM aan. Ongeveer 64\% van de patiënten met laesies waarbij de vestibulaire subnuclei van de thalamus betrokken zijn en $44 \%$ van de patiënten met laesies in de vestibulaire cortex (insulaire, superieure temporale en transverse temporale gyri) vertonen binoculaire pathologische statische SVV afwijkingen welke voornamelijk contraversief zijn. Niet enkel centrale of perifere neurovestibulaire aandoeningen veroorzaken pathologische statische SVV deviaties. Pathologische statische SVV deviaties worden ook teruggevonden in $67 \%$ van de patiënten met verticale externe oogspierparesen ten gevolge van ipsilaterale IIIe en IVe hersenzenuw (respectievelijk nervus oculomotorius en nervus trochlearis) verlammingen. De pathologische statische SVV afwijkingen waren echter enkel monoculair en beperkt tot het aangedane oog in het acute stadium tot 3 maanden erna. Daarna gaan de pathologische monoculaire statische SVV afwijkingen geleidelijk over naar het contralaterale niet-aangedane oog ten gevolge van compensatie mechanismen ${ }^{29}$. Het grote verschil tussen de supranucleaire en infranucleaire oculomotore aandoeningen is dat statische SVV afwijkingen in infranucleaire aandoeningen beperkt zijn tot het aangedane oog in de acute setting en overgaan naar het niet-aangedane oog in de chronische setting na 3 maanden. De binoculaire statische SVV resultaten zijn normaal in alle patiënten met infranucleaire oculomotore aandoeningen in tegenstelling tot de patiënten met supranucleaire aandoeningen waarbij tot $94 \%$ van de patiënten met een herseninfarct van de hersenstam afwijkende binoculaire statische SVV resultaten vertoonden. Tevens kan een bilaterale nervus trochlearis parese worden gelokaliseerd middels statische SVV testen welke een bilaterale monoculaire statische SVV tilt vertoonden met tegengestelde richting in 
combinatie met een normale binoculaire statische SVV meting. Pathologische statische SVV afwijkingen in supranucleaire laesies zijn namelijk altijd gericht naar dezelfde zijde $^{25,29}$. Samenvattend geven additionele statische SVV testen waarbij zowel monoculaire als binoculaire metingen worden gecombineerd, dus belangrijke additionele diagnostische informatie.

In onze studie toonden we eveneens aan dat statische SVV metingen werden beïnvloed in de richting van de uitgangshoek, echter niet door de grootte van de hoek zelf (voor deviaties van de hoek groter dan 10 graden). Onze bevindingen zijn in overeenstemming met eerder gepubliceerde studies in de wetenschappelijke literatuur ${ }^{24,31}$, echter uitgangshoek deviaties kleiner dan 8 graden hebben geleidelijk minder invloed op de uiteindelijke statische SVV resultaten. In onze studie en in de literatuur worden statische SVV afwijkingen tussen de 10 en 20 graden frequent gezien. Daardoor wordt geadviseerd om een uitgangshoek met een begindeviatie van 20 graden te nemen en om de uitgangshoeken met de richting van de klok en daartegen in gelijk maar wel ad random te verdelen bij herhaalde metingen (ten gevolge van de bias van de resultaten in de richting van de uitgangshoek). Beide aanbevelingen zijn geïncorporeerd in het uiteindelijke voorstel voor het statische SVV test protocol.

Vergelijkbaar met de VEMP testen vertoonden ook de statische SVV testen bij de gezonde vrijwilligers een individuele test her-test variabiliteit die stabiel was op groepsniveau zonder statistisch significante verschillen. De resultaten van alle gezonde vrijwilligers bleven echter binnen de normale grenzen zowel in de test als her-test metingen. Men kan zich echter afvragen of dit ook geldt voor patiënten met afwijkende statische SVV metingen. Statische SVV metingen neigen echter te normaliseren over het beloop van de tijd bijvoorbeeld bij herseninfarcten in de hersenstam ${ }^{25,28}$ en gaan geleidelijk over van het paretische oog bij infranucleaire oculomotore aandoeningen in de acute fase naar de contralaterale zijde na een periode van 3 maanden ${ }^{29}$. Daardoor adviseren we toekomstig onderzoek naar de test her-test variabiliteit bij patiënten met afwijkende statische SVV metingen, echter om de periode tussen de testen zeer kort te houden met een maximum van enkele dagen gezien het dynamische karakter van de statische SVV afwijkingen.

De toepassing van statische SVV testen buiten gespecialiseerde derdelijns duizeligheidsklinieken wordt bemoeilijkt door de hoge kosten van gespecialiseerde apparatuur. Zwergal et al. ${ }^{32}$ bestudeerden een draagbare emmer-versie van de statische SVV test welke zelf gemaakt kan worden tegen lage kosten, zie figuur 1 van dit hoofdstuk. De conversie van een huis-tuin-en-keuken emmer duurt minder dan een uur en kost ongeveer 5 US dollars. De auteurs vergeleken deze emmer-versie van de 
statische SVV test met de conventionele statische SVV test en vonden een zeer goede inter- en intra-test betrouwbaarheid (Pearson correlatie coëfficiënten van respectievelijk 0.89-0.90 and 0.92) zowel in gezonde proefpersonen als in patiënten met acute neurovestibulaire aandoeningen. Brodsky et al..$^{33}$ onderzochten recent een smartphone versie van de statische SVV emmer-versie bij pediatrische patiënten met een verdenking op een neurovestibulaire aandoening. Deze subjectieve verticaliteit applicatie op de smartphone koste ongeveer 18 US dollars en werd vergeleken met de conventionele statische SVV test. Een matige Pearson correlatie coëfficiënt van 0.43 werd gevonden, echter ondanks de matige correlatie was de SVV een nuttige screeningsmethode voor acute neurovestibulaire aandoeningen in kinderen (sensitiviteit 75\%, specificiteit $100 \%$, positief voorspellende waarde van $100 \%$ en negatief voorspellende waarde van $92 \%)$. Ondanks de veelbelovende resultaten van de bovenstaande onderzoeken is verder onderzoek in grotere groepen aangewezen waarbij de (smartphoneondersteunende) emmer-versie van de statische SVV test wordt vergeleken met conventioneel statisch SVV onderzoek om de bovenstaande bevindingen te bevestigen.
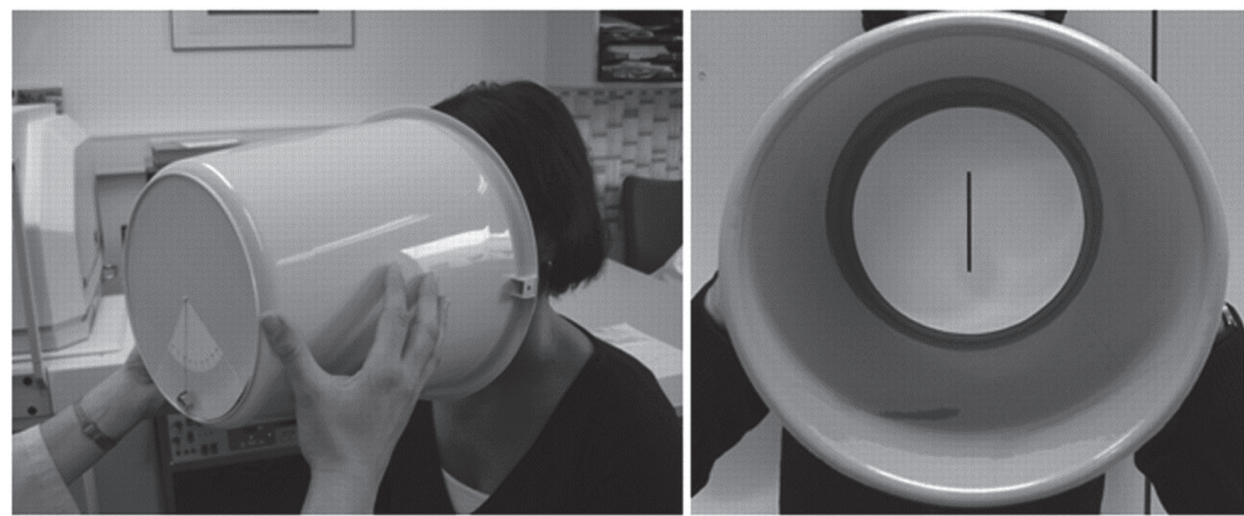

Figuur 1

Emmer-versie van de statische SVV test, bewerking van Zwergal et al. (2009) ${ }^{32}$ 


\section{DEEL TWEE}

\section{Neurovestibulaire analyse en vallen bij personen met de ziekte van Parkinson en atypische parkinsonismen. De resultaten van de baseline studie en 1-jaars follow-up.}

\section{Samenvatting hoofdstuk 5}

Terugkerende valepisoden zijn een groot probleem bij personen met de ziekte van Parkinson en nog meer uitgesproken bij personen met atypische parkinsonismen. Ongeveer 50\% van personen met de ziekte van Parkinson vallen regelmatig en ongeveer $70 \%$ valt minimaal eens per jaar ${ }^{34-37}$. Personen met de ziekte van Parkinson hebben 2.2x meer kans op fracturen in het algemeen en 3-4x meer kans op heupfracturen in vergelijking met een gezonde groep proefpersonen met dezelfde leeftijd. Tevens hebben personen met de ziekte van Parkinson over het algemeen hogere mortaliteitskansen en hogere risico's op peri- en postoperatieve complicaties gedurende de behandeling van deze fracturen ${ }^{38}$. Onlangs werd een praktische consensusafspraak gepubliceerd met betrekking tot de risico factoren voor vallen bij personen met de ziekte van Parkinson en adviezen voor de behandeling hiervan ${ }^{39}$. Vestibulaire dysfunctie werd echter niet besproken als een van de mogelijke oorzaken voor het vallen wat verassend is, aangezien het vestibulaire systeem onmisbaar is in het bewaren van de balans door multimodale sensore input te verwerken (zijnde de perifeer vestibulaire, proprioceptieve en visuele input) en om vervolgens de motore respons bij te sturen door middel van de vestibulospinale reflexen ${ }^{37}$. Hoofdstuk 5 toont de resultaten van een casecontrol studie waarbij de functie van het vestibulaire systeem bij 25 gezonde proefpersonen systematisch werd onderzocht en vergeleken met 30 personen met de ziekte van Parkinson en 14 personen met atypische parkinsonismen (6 personen met multipele systeem atrofie, 3 met progressieve supranucleaire verlamming en 5 met vasculair parkinsonisme) $)^{37}$. Ongeveer $90 \%$ van de personen met de ziekte van Parkinson (27 van de 30) en $100 \%$ van de personen met atypische parkinsonismen (alle 14) vertoonden bij het neurofysiologisch onderzoek tekenen van vestibulaire dysfunctie met voornamelijk een centraal vestibulair dysfunctie profiel (bij de VEMP, SVV en videonystagmografie metingen met additionele calorische stimulatie en draaistoelonderzoek). Daarnaast waren neurofysiologische afwijkingen bij de vestibulaire metingen sterk geassocieerd met een toegenomen risico voor vallen (wanneer de resultaten van vallende personen met de ziekte van Parkinson en atypische parkinsonismen werden vergeleken met de resultaten van niet vallende personen, $\mathrm{P} \leq 0.001$ ). 
Afwijkende neurovestibulaire functie testen worden opvallend vaak in zowel personen met de ziekte van Parkinson en atypische parkinsonismen gezien (90\% van de personen met de ziekte van Parkinson en 100\% bij atypische parkinsonismen). Ongeveer 66\% van de patiënten met een milde vorm van de ziekte van Parkinson heeft afwijkende functie testen, waarbij deze testen bij $96 \%$ en $100 \%$ afwijkend zijn bij patiënten met een respectievelijk een matige tot ernstige vorm van de ziekte. Daarbij kan worden geconcludeerd dat afwijkende neurovestibulaire functie testen vaak worden aangetroffen bij personen met een mild stadium van de ziekte van Parkinson, maar dat de afwijkingen toenemend aanwezig zijn bij meer gevorderde stadia van de ziekte. Tevens waren de personen met atypische parkinsonismen ernstiger aangedaan in vergelijking met personen met de ziekte van Parkinson (respectievelijk Hoehn en Yahr stadia $2.9 \pm 0.8$ en $2.3 \pm 0.7 ; \mathrm{P}=0.0152$ ). Personen met neurovestibulaire en/of andere neurologische aandoeningen (anders dan de ziekte van Parkinson of atypische parkinsonismen) in hun voorgeschiedenis werden geëxcludeerd. Tevens had geen van de proefpersonen relevante medische klachten anamnestisch danwel symptomen bij klinisch neurologisch en neuro-otologisch onderzoek welke suggestief waren voor een andere onderliggende neurovestibulaire stoornis. Logischerwijs konden niet alle bestaande andere (centrale) neurovestibulaire stoornissen middels deze onderzoeken worden geëxcludeerd, voornamelijk door de afwezigheid van recente cerebrale MRI beeldvormende studies. Gezien echter het ontbreken van relevante klachten anamnestisch danwel symptomen bij neurologisch en neuro-otologisch onderzoek waren hiervoor op klinische gronden geen aanwijzingen.

De abnormale neurovestibulaire functie testen hadden voornamelijk een centraal vestibulair dysfunctie profiel in $78-93 \%$ van de personen met respectievelijk de ziekte van Parkinson en atypische parkinsonismen. Slechts één persoon met de ziekte van Parkinson (4\%) had een profiel suggestief voor een perifere vestibulaire aandoening waarbij de overige profielen niet lokaliserend waren. Tevens hadden $57-79 \%$ van de personen met respectievelijk atypische parkinsonismen en de ziekte van Parkinson anamnestisch geen klachten van duizeligheid of vertigo. Men kan zich echter afvragen of de gevonden neurovestibulaire functie afwijkingen bij deze groep inderdaad asymptomatisch zijn. Ongeveer 10-18\% van de personen met atypische parkinsonismen en de ziekte van Parkinson hebben neurovestibulaire functie afwijkingen bij neurofysiologische testen als de enige identificeerbare oorzaak voor vallen (na exclusie van orthostatische hypotensie, posturale instabiliteit, freezing of gait en matige-tot-ernstige cognitieve problemen) ${ }^{37}$. Helaas konden niet alle overige mogelijke oorzaken voor vallen behoudens de vestibulaire dysfunctie bij de bovenstaande patiënten worden uitgesloten (zoals bijvoorbeeld milde orthostatische hypotensie welke wisselend aanwezig kan zijn bij routine testen) ${ }^{39,40}$, echter wel de 
overige meest relevante oorzaken. Daarnaast zijn vestibulaire afwijkingen bij neurofysiologische testen sterk gecorreleerd met een verhoogd risico voor vallen wanneer vallende personen met de ziekte van Parkinson en atypische parkinsonismen werden vergeleken met niet-vallende personen $(\mathrm{P} \leq 0.001)$. Uit de bovenstaande gegevens werd geconcludeerd dat neurovestibulaire dysfunctie zich uitende in afwijkende neurofysiologische vestibulaire testen veel voorkomen bij personen met de ziekte van Parkinson en atypische parkinsonismen en sterk geassocieerd zijn met een verhoogd valrisico (wat tot op heden niet eerder in de wetenschappelijke literatuur werd gerapporteerd $)^{37}$. Met betrekking tot de bovenstaande studie moet worden gemeld dat de bestudeerde patiënten groepen relatief klein en heterogeen zijn (met name met betrekking tot de atypische parkinsonismen). De resultaten van deze studie moeten daarom voorzichtig worden geïnterpreteerd en voornamelijk worden gezien als hypothese genererend voor toekomstig onderzoek waarbij bevestiging van de resultaten in grotere patiëntengroepen wordt geadviseerd. Daarbij is het momenteel niet bekend of de sterk geassocieerde neurovestibulaire test afwijkingen direct een causaal verband hebben met vallen danwel een epifenomeen zijn (bijvoorbeeld door deconditionering ten gevolge van het vallen). Dopaminerge medicatie lijkt echter geen goede verklaring te zijn voor de gevonden neurovestibulaire test afwijkingen aangezien studies door de auteurs Pötter-Nerger et al. . $1,42^{4}$ aantoont dat levodopa mogelijks vestibulaire test afwijkingen partieel verbeterd. Het centrale vestibulaire systeem is echter een van de belangrijke systemen in het behoudt van balans door de integratie van multimodale sensore informatie (perifeer vestibulaire, visuele en proprioceptieve input) en om vervolgens de uitgaande motore respons te moduleren middels de vestibulospinale reflexen. Op basis van de bovenstaande redenering kan men puur hypothetisch gebaseerd op theoretische fysiologische evidentie concluderen dat vestibulaire dysfunctie causaal verantwoordelijk is voor vallen als een mogelijke oorzaak en niet gebaseerd is op een epifenomeen.

De centrale vestibulaire test afwijkingen in personen met de ziekte van Parkinson bestaan voornamelijk uit afwijkende geëvoceerde potentialen testen (VEMPs en BAEPs), welke significant verlengde latenties (p13, n1 en inter-top III-V latenties) lieten zien aan de symptomatische zijde van de hersenstam in vergelijking met gezonde proefpersonen $(0.003 \leq \mathrm{P} \leq 0.019)$. Vergelijking van de meetresultaten van de asymptomatische zijde van personen met de ziekte van Parkinson en gezonde proefpersonen of de kant-tot-kant vergelijkingen binnen de personen met de ziekte van Parkinson lieten geen significant afwijkende waarden zien met uitzondering van de p13 latentie van de cervicale VEMP kant-tot-kant vergelijking $(P=0.020)$. Deze resultaten wijzen op een asymmetrische hersenstam betrokkenheid in het neurodegeneratieve proces welke voornamelijk de aangedane zijde betreft en vervolgens 
via de aangedane zenuwvezelbanen mee verloopt (de aangedane hersenstam zijde is ipsilateraal ten opzichte van de patiënt zijn rusttremoren, rigiditeit en bradykinesie op het niveau van de pontomedullaire overgang onder het niveau van de vestibulaire kernen; de aangedane zenuwvezelbanen kruisen naar de contralaterale hersenstam zijde boven het niveau van de vestibulaire kernen samen met de decussatio van de fasciculus longitudinalis medialis). Pötter-Nerger et al. ${ }^{41}$ laten enkel asymmetrische afwijkingen van de oculaire VEMP testen zien aan de symptomatische hersenstam zijde waarbij de cervicale VEMP deze asymmetrische afwijkingen niet liet zien. Het voornaamste verschil tussen de studie door Pötter-Nerger et al. ${ }^{41}$ en onze studie is de grootte van de populatie (30 personen met de ziekte van Parkinson in onze studie versus 13 personen in de studie van Pötter-Nerger et al. $\left.{ }^{41}\right)$. Kleinere verschillen tussen groepen worden namelijk sneller gedetecteerd in grotere studie populaties. Onze studie demonstreert eveneens dat niet enkel centrale vestibulaire banen zijn aangedaan aan de symptomatische zijde van de hersenstam, maar dat dit tevens geldt voor de centrale auditieve banen wat wordt geillustreerd door latentie verlenging van de BAEP intertop III-V latenties aan de symptomatische zijde.

Zoals eerder vermeld bestaan de BAEP en VEMP afwijkingen in onze studie voornamelijk uit sterk verlengde cervicale VEMP p13 latenties, oculaire VEMP n1 latenties en BAEP inter-top III-V latenties (p13 134\%, n1 128\% en III-V inter-top latenties $120 \%$ van de bovenste limiet van normaal) met relatief gespaarde amplitudes ${ }^{37}$. Zoals bediscussieerd in paragraaf 1.1 van dit hoofdstuk worden op theoretische gronden asymmetrisch sterk verlengde geëvoceerde potentialen latenties met relatief gespaarde amplitudes mogelijks verklaard door primair demyeliniserende aandoeningen. Het pathofysiologisch onderscheid tussen axonale en demyeliniserende centraal neurologische neurodegeneratieve aandoeningen gebaseerd op de resultaten van geëvoceerde potentialen metingen is puur speculatief en tot zover onze kennis reikt nog niet eerder bestudeerd. Op dit moment zijn er dan ook geen neurofysiologische criteria voor deze pathofysiologische differentiatie voor aandoeningen van het centrale zenuwstelsel, zoals deze er bijvoorbeeld wel zijn voor de differentiatie van perifere neuropathieën. Meer wetenschappelijk onderzoek met betrekking tot deze differentiatie tussen axonale en demyeliniserende centrale zenuwstelsel aandoeningen gebaseerd op geëvoceerde potentialen metingen lijken dan ook interessant omdat dit belangrijke inzichten kan geven in de onderliggende pathofysiologische mechanismen van centrale zenuwstelsel aandoeningen waarvan dit tot op heden niet goed bekend is.

De ziekte van Parkinson is pathofysiologisch gedefinieerd bij de aanwezigheid van abnormale alfa-synucleïne eiwit aggregaties in neuronen en gliale cellen; en door de aanwezigheid van neurodegeneratie van specifieke hersenregio's (cholinerge 
pedunculopontiene nucleus, dopaminerge substantia nigra en de ventrale tegmentale area). Deze eiwit aggregaties worden als volgt genoemd: a) Lewy bodies in neuronale perikarya, b) Lewy neurieten in de axonen en dendrieten, c) coiled bodies in aangedane oligodendrocyten ${ }^{43,44}$. Tevens wordt multisysteem atrofie (MSA, een vorm van atypisch parkinsonisme) gekarakteriseerd door neuronale en gliale alfa-synucleïne aggregaten, echter waarbij er in het algemeen wordt aangenomen dat deze eveneens in contrast tot de ziekte van Parkinson ook aanwezig zijn in hersenstam nuclei ${ }^{-44}$. Seidel et al. ${ }^{43}$ demonstreerden met hun neuropathologische studie dat Lewy bodies en neurieten eveneens aanwezig zijn in alle hersenstam craniale zenuwkernen, premotore, oculomotore, precerebellaire en vestibulaire nucleï bij patiënten met de ziekte van Parkinson. Dit was in contrast met de algemene aanname dat deze afwijkingen beperkt waren tot personen met MSA. Daarbij werden er in de bovenvermelde studie ook Lewy neurieten en coiled bodies aangetroffen in alle hersenstam zenuwvezelbanen. Deze afwijkingen zijn mogelijks het resultaat van een transneuronale ziekte verspreiding via de anatomische baansystemen ten gevolge van verstoorde intra-axonale transport mechanismen ${ }^{43}$. Wanneer we de neuropathologische en neurofysiologische wetenschappelijke kennis van de ziekte van Parkinson tot op heden combineren, lijkt een transneuronale ziekteprogressie via de anatomische zenuwvezelbanen het meest waarschijnlijk welke de hersenstam asymmetrisch aantast aan de symptomatische zijde van de patiënt. Asymmetrische aantasting van de hersenstam onder het niveau van het mesencefalon (substantia nigra) is tot op heden nog niet bevestigd middels neuropathologische studies en lijkt een interessant gebied voor toekomstig onderzoek. De afwijkingen welke neuropathologisch worden gevonden suggereren een neurodegeneratief proces met een primair axonaal kenmerk $^{43}$, echter de neurofysiologische afwijkingen (fors verlengde latenties met relatief gespaarde amplitudes) passen beter bij een primair demyeliniserende neurodegeneratieve component. Recent dierexperimenteel onderzoek in verschillende diermodellen suggereren dat synaptische dysfunctie eveneens een vroeg en belangrijke component is in de ziekte van Parkinson ${ }^{45}$, welke eveneens de neurofysiologische afwijkingen ten dele kan verklaren. Toekomstige neuropathologische hersenstam studies bij de ziekte van Parkinson zouden daardoor tevens gefocust moeten zijn op de myelinisatie van aangedane zenuwvezelbanen welke Lewy neurieten en coiled bodies bevatten met de primaire vraag of begeleidende demyelinisatie een belangrijk kenmerk van de neurodegeneratie bij deze aandoening is. Natuurlijk zijn er ook meer toekomstige studies in mensen noodzakelijk omtrent de analyse naar mogelijke synaptische dysfunctie bij de ziekte van Parkinson, welke bij diermodellen werd teruggevonden. 


\section{Samenvatting hoofdstuk 6}

De één-jaar follow-up resultaten van de case-control baseline studie, zoals hierboven beschreven in paragraaf 2 van dit hoofdstuk en welke in hoofdstuk 5 in meer detail zijn bediscussieerd, worden weergegeven in hoofdstuk 6 . Alle 25 gezonde vrijwilligers, 14 personen met atypische parkinsonismen en 30 personen met de ziekte van Parkinson werden telefonisch benaderd voor een interview één jaar volgend op de baseline onderzoeken. Slechts 1 patiënt met een atypisch parkinsonisme (MSA) kon niet meer telefonisch worden bereikt ondanks herhaaldelijke oproepen en was daarom lossed to follow-up (attrition bias van slechts $1,4 \%$ ). Alle deelnemers werden ondervraagd met betrekking tot hun val frequentie gebaseerd op de valkalenders en over hun subjectief vertrouwen in de eigen balans (middels de $\mathrm{ABC}-16$ vragenlijst). De resultaten na één jaar follow-up werden met de baseline resultaten vergeleken. De voornaamste onderzoeksvraag was of neurovestibulaire laboratorium testen (verricht bij aanvang van het onderzoek) toekomstige valepisoden bij personen met de ziekte van Parkinson en atypische parkinsonismen kunnen voorspellen. Cervicale en oculaire VEMP testen in combinatie met klinische testen voor posturale instabiliteit zijn de beste voorspellers van toekomstige valepisoden met een sensitiviteit van $100 \%$, positief voorspellende waarde van $68,2 \%$ bij slechts één afwijkende VEMP test en 83,3\% wanneer beide VEMP testen afwijkend zijn. De valfrequentie zowel bij baseline als na één jaar follow-up was statistisch significant hoger en het subjectieve vertrouwen in de eigen balans (volgens de $\mathrm{ABC}-16$ vragenlijst) was statistisch significant lager bij zowel Parkinson patiënten en patiënten met atypische parkinsonismen in vergelijking met gezonde vrijwilligers. Concluderend kan men stellen dat afwijkende VEMP testen geassocieerd zijn met een toegenomen risico op toekomstige valepisoden bij Parkinson patiënten en patiënten met atypische parkinsonismen met posturale instabiliteit in de afwezigheid van freezing of gait ${ }^{46}$.

Een screenings test is enkel bruikbaar in de klinische praktijk wanneer deze zowel een hoge sensitiviteit heft, als een positief voorspellende waarde. Wetende dat freezing of gait een grote risicofactor voor toekomstige valepisoden is ${ }^{47,48}$, bestudeerden we de associatie tussen freezing bij de baseline metingen en het toekomstige valrisico. In onze studie bleek de sensitiviteit van freezing of gait slechts $46,9 \%$ (slechts 7 van de 15 vallende patiënten warden gedetecteerd), echter waarbij dit symptoom wel de hoogste positief voorspellende waarde had van namelijk 87,5\% (bij kwalitatieve beoordeling van freezing middels het beoordelen van rechtdoor lopen en vlug en bloc draaien in beide richtingen). Voornamelijk de zeer beperkte sensitiviteit maakt het 
testen van freezing of gait en zich een slechte screeningstest voor toekomstige valepisoden (aangezien ruim de helft van de potentieel vallende patiënten zal worden gemist). Het klinisch testen van de houdingsreflexen voor posturale instabiliteit (middels de retropulsietest) heeft een sensitiviteit van 100\% voor toekomstige valepisoden in Parksinon patiënten en patiënten met atypische parkinsonismen, echter de positief voorspellende waarde is slechts $46,9 \%$. Dus het testen van de houdingsreflexen alleen is niet bruikbaar als een screeningstest aangezien de helft van de patiënten met afwijkende houdingsreflexen het komende jaar niet zal vallen. De combinatie van zowel de cervicale en oculaire VEMP testen met klinische testen van de posturale stabiliteit lijken de ideale combinatie voor een screeningstest (met een sensitiviteit van $100 \%$ en positief voorspellende waarde van $68,2 \%$ wanneer slechts één VEMP test afwijkend is en van 83,3\% wanneer beide VEMP testen afwijkend zijn) voor het voorspellen van toekomstige valepisoden bij Parkinson patiënten en patiënten met atypische parkinsonismen. De aanwezigheid van freezing of gait is echter zo'n sterke, maar insensitieve, voorspeller voor vallen dat aanvullende VEMP testen bij Parkinson patiënten en patiënten met atypische parkinsonismen met het symptoom freezing geen toegevoegde waarde hebben (alle patiënten met freezing of gait hadden eveneens posturale instabiliteit en afwijkende VEMP testen). Concluderend kan worden gesteld dat cervicale en oculaire VEMP testen aanvullende informatie kunnen geven over het toekomstige valrisico in geselecteerde personen met de ziekte van Parkinson en atypische parkinsonisme (namelijk bij personen zonder de aanwezigheid van freezing of gait ${ }^{46}$. De bovenstaande resultaten moeten echter voorzichtig worden geïnterpreteerd aangezien de patiënten groepen kleine aantallen bevatten met voornamelijk een heterogene populatie in de patiënten groep met atypische parkinsonismen.

Men kan er echter over speculeren of deze additionele informatie met betrekking tot het toekomstige valrisico bij personen met de ziekte van Parkinson of met atypische parkinsonismen zal resulteren in andere val preventieve maatregelen aangezien patiënten met een verstoorde posturale stabiliteit reeds een hoog valrisico hebben. Meerdere meta-analyses en Cochrane reviews met betrekking tot het effect van fysiotherapie bij Parkinson patiënten rapporteren enkel korte-termijn statistisch significante, maar klinisch bescheiden, positieve effecten ten aanzien van balansgerelateerde taken en verbetering van het looppatroon. Er is echter geen wetenschappelijke evidentie dat het valrisico eveneens afneemt onder deze therapie ${ }^{46-52}$. Eén Cochrane review ${ }^{49}$ vergeleek de verschillende vormen van fysiotherapie en concludeerde dat een formele kwalitatieve vergelijking van de therapieën niet mogelijk is gezien de uitgesproken heterogeniteit van de studies, kleine patiënten aantallen, methodologische tekortkomingen en het risico van publicatie bias. Daardoor is er 
geen wetenschappelijke evidentie om één vorm van fysiotherapie te verkiezen boven een andere. Prospectieve studies met een hoge kwaliteit en lange follow-up zijn daarom nodig om het effect van fysiotherapie bij zowel personen met de ziekte van Parkinson of met atypische parkinsonismen te beoordelen met betrekking tot de afname van het valrisico en om te beoordelen welke vorm van therapie superieur is om dit te bereiken boven een andere. Deze studies zouden evidence-gebaseerde richtlijnen voor fysiotherapie bij de ziekte van Parkinson moeten volgen en zouden idealiter moeten worden uitgevoerd door therapeuten met ervaring in behandeling van personen met de ziekte van Parkinson ${ }^{53-56}$.

Een recente case-studie beschreef de motore en niet-motore effecten van herhaalde calorische vestibulaire stimulatie bij een patiënt met de ziekte van Parksinon ${ }^{47}$. De motore en niet-motore scores (Montreal cognitieve beoordeling, MOCA; ziekenhuis depressie schaal, depressie deel van de HADS vragenlijst; en de Epworth slaperigheids score) verbeterden allen duidelijk zowel gedurende de herhaalde calorische stimulatie en na 5 maanden follow-up na het staken hiervan. Deze verbeteringen werden niet gezien gedurende de eerste fase van de studie welke bestond uit placebo stimulatie. Tevens laten andere recente studies bij personen met de ziekte van Parkinson voorlopig bewijs zien dat (stochastische) galvanische vestibulaire stimulatie mogelijks posturale instabiliteit verbetert, correctieve posturale responsen versterkt, voorover gebogen houding corrigeert en boven alles veilig is voor gebruik op de relatief korte termijn ${ }^{58-62}$. Deze studies bestaan echter uit kleine case-studies of kleine case-series. Naar onze mening zouden deze studies gezien moeten worden als proof-of-concept bewijs en zouden deze herhaald moeten worden in grotere dubbel blind gerandomiseerde studies om hun toegevoegde therapeutische waarde in de preventie van valepisoden en verbetering van zowel motore als non-motore symptomen bij personen met de ziekte van Parkinson als atypische parkinsonismen definitief vast te leggen.

Op dit moment is er geen bewijs om standaard VEMP testen uit te voeren bij personen met de ziekte van Parkinson of met atypische parkinsonismen aangezien het geen therapeutische consequenties heeft. Vestibulaire laboratorium testen (VEMP, SVV en/of videonystagmografie met calorisatie en draaistoelstimulatie) kunnen echter wel erg behulpzaam zijn in wetenschappelijke studies in het ophelderen van de onderliggende fysiologische veranderingen secundair aan bijvoorbeeld herhaalde calorische therapeutische stimulatie of stochastische vestibulaire stimulatie zoals hierboven beschreven in de mogelijke preventie van valepisoden. 


\section{Referenties}

1. Curthoys, I. S. A critical review of the neurophysiological evidence underlying clinical vestibular testing using sound, vibration and galvanic stimuli. Clin. Neurophysiol. 121, 132-144 (2010).

2. Eleftheriadou, A. \& Koudounarakis, E. Vestibular-evoked myogenic potentials eliciting: An overview. European Archives of Oto-Rhino-Laryngology 268, 331-339 (2011).

3. Rosengren, S. M., Welgampola, M. S. \& Colebatch, J. G. Vestibular evoked myogenic potentials: Past, present and future. Clin. Neurophysiol. 121, 636-651 (2010).

4. Rosengren, S. \& Kingma, H. New perspectives on vestibular evoked myogenic potentials. Curr. Opin. Neurol. 26, 74-80 (2013).

5. Venhovens, J., Meulstee, J. \& Verhagen, W. Vestibular Evoked Myogenic Potentials (VEMPs) in Central Neurological Disorders. Clin. Neurophysiol. 127, 40-49 (2016).

6. Colebatch, J. G. Sound conclusions? Clin. Neurophysiol. 121, 124-126 (2010).

7. Curthoys, I. S. A balanced view of the evidence leads to sound conclusions. A reply to J.G. Colebatch 'Sound conclusions?'. Clin. Neurophysiol. 121, 977-8 (2010).

8. Papathanasiou, E. S. Ocular vestibular evoked myogenic potentials (OVEMPs): saccule or utricle? Clin. Neurophysiol. 123, 216 (2012).

9. Papathanasiou, E. S. \& Papacostas, S. S. Vestibular evoked myogenic potentials: the fuzzy picture of different stimulation types is beginning to come into focus. Clin. Neurophysiol. 124, 1926-7 (2013).

10. Todd, N. The ocular vestibular evoked myogenic potential (OVEMP), ten years old. Clin. Neurophysiol. 125, 439-41 (2014).

11. Venhovens, J., Meulstee, J. \& Verhagen, W. I. M. Ocular and Cervical Vestibular Evoked Myogenic Potentials (VEMPs) in healthy volunteers: the intra-, interobserver, and the test re-test reliability. J. Vestib. Res. 25, 161-7 (2015).

12. Brantberg, K. Vestibular evoked myogenic potentials (VEMPs): Usefulness in clinical neurotology. Seminars in Neurology 29, 541-547 (2009).

13. Yew, A. et al. Characteristics and management of superior semicircular canal dehiscence. J. Neurol. Surg. B. Skull Base 73, 365-70 (2012).

14. Zuniga, M. \& Janky, K. Ocular vs. cervical VEMPs in the diagnosis of superior semicircular canal dehiscence syndrome. Otol. Neurotol. Off. Publ. Am. Otol. Soc. Am. Neurotol. Soc. [and] Eur. Acad. Otol. Neurotol. Neurotol. 34, 121-126 (2013).

15. Tankisi, H., Pugdahl, K., Johnsen, B. \& Fuglsang-Frederiksen, A. Correlations of nerve conduction measures in axonal and demyelinating polyneuropathies. Clin. Neurophysiol. 118, 2383-2392 (2007).

16. Chung, T., Prasad, K. \& Lloyd, T. E. Peripheral neuropathy. Clinical and electrophysiological considerations. Neuroimaging Clinics of North America 24, 49-65 (2014).

17. Nuwer, M. R. Fundamentals of evoked potentials and common clinical applications today. Electroencephalography and Clinical Neurophysiology 106, 142-148 (1998).

18. Lascano, A. M., Lalive, P. H., Hardmeier, M., Fuhr, P. \& Seeck, M. Clinical evoked potentials in neurology: a review of techniques and indications. J. Neurol. Neurosurg. Psychiatry jnnp-2016314791 (2017). doi:10.1136/jnnp-2016-314791

19. O.N., M. Brainstem auditory evoked potentials. J. Clin. Neurophysiol. 11, 319-342 (1994).

20. Vanspauwen, R., Wuyts, F. L. \& Van De Heyning, P. H. Validity of a new feedback method for the VEMP test. Acta Otolaryngol. 126, 796-800 (2006).

21. Vanspauwen, R., Wuyts, F. L. \& Van De Heyning, P. H. Vestibular evoked myogenic potentials: Test-retest reliability and normative values obtained with a feedback method for the sternocleidomastoid muscle contraction. J. Vestib. Res. Equilib. Orientat. 19, 127-135 (2009).

22. Nguyen, K. D., Welgampola, M. S. \& Carey, J. P. Test-retest reliability and age-related characteristics of the ocular and cervical vestibular evoked myogenic potential tests. Otol. Neurotol. 31, 793-802 (2010).

23. Venhovens, J., Meulstee, J. \& Verhagen, W. I. M. Static Subjective Visual Vertical in Healthy Volunteers: The Effects of Different Preset Angle Deviations and Test-Retest Variability. Neuroophthalmology. 40, 113-119 (2016). 
24. Baccini, M., Paci, M., Del Colletto, M., Ravenni, M. \& Baldassi, S. The assessment of subjective visual vertical: comparison of two psychophysical paradigms and age-related performance. Atten. Percept. Psychophys. 76, 112-22 (2014).

25. Brandt, T. \& Dieterich, M. Vestibular syndromes in the roll plane: topographic diagnosis from brainstem to cortex. Ann. Neurol. 36, 337-47 (1994).

26. Tarnutzer, a a, Bockisch, C., Straumann, D. \& Olasagasti, I. Gravity dependence of subjective visual vertical variability. J. Neurophysiol. 102, 1657-1671 (2009).

27. Yelnik, A. P. et al. Perception of verticality after recent cerebral hemispheric stroke. Stroke 33, 2247-2253 (2002).

28. Dieterich, M. \& Brandt, T. Ocular torsion and tilt of subjective visual vertical are sensitive brainstem signs. Ann. Neurol. 33, 292-299 (1993).

29. Dieterich, M. \& Brandt, T. Ocular torsion and perceived vertical in oculomotor, trochlear and abducens nerve palsies. Brain 116, 1095-1104 (1993).

30. Brandt, T., Dieterich, M. \& Danek, A. Vestibular cortex lesions affect the perception of verticality. Ann. Neurol. 35, 403-412 (1994).

31. Pagarkar, W., Bamiou, D.-E., Ridout, D. \& Luxon, L. M. Subjective visual vertical and horizontal: effect of the preset angle. Arch. Otolaryngol. Head. Neck Surg. 134, 394-401 (2008).

32. Zwergal, A. et al. A bucket of static vestibular function. Neurology 72, 1689-1692 (2009).

33. Brodsky, J. R., Cusick, B. A., Kawai, K., Kenna, M. \& Zhou, G. Peripheral vestibular loss detected in pediatric patients using a smartphone-based test of the subjective visual vertical. Int. J. Pediatr. Otorhinolaryngol. 79, 2094-2098 (2015).

34. Wood, B. H., Bilclough, J. A., Bowron, A. \& Walker, R. W. Incidence and prediction of falls in Parkinson's disease: a prospective multidisciplinary study. J. Neurol. Neurosurg. Psychiatry 72, $721-$ 725 (2002).

35. Bloem, B. \& Bhatia, K. in Clinical disorders of balance, posture and gait. (ed. Bronstein AM, Brandt T, Nutt JG, W. M.) 173-206 (Arnold: London, 2004).

36. Grimbergen, Y. A. M., Munneke, M. \& Bloem, B. R. Falls in Parkinson's disease. Curr. Opin. Neurol. 17, 405-415 (2004).

37. Venhovens, J., Meulstee, J., Bloem, B. R. \& Verhagen, W. I. M. Neurovestibular analysis and falls in Parkinson's disease and atypical parkinsonism. Eur. J. Neurosci. 43, 1636-46 (2016).

38. Critchley, R. J., Khan, S. K., Yarnall, A. J., Parker, M. J. \& Deehan, D. J. Occurrence, management and outcomes of hip fractures in patients with Parkinson's disease. Br. Med. Bull. 115, 135-42 (2015).

39. van der Marck, M. A. et al. Consensus-based clinical practice recommendations for the examination and management of falls in patients with Parkinson's disease. Parkinsonism and Related Disorders 20, 360-369 (2014).

40. Thijs, R. D., Bloem, B. R. \& Dijk, J. G. Falls, faints, fits and funny turns. J. Neurol. 256, 155-167 (2009).

41. Pötter-Nerger, M., Govender, S., Deuschl, G., Volkmann, J. \& Colebatch, J. G. Selective changes of ocular vestibular myogenic potentials in Parkinson's disease. Mov. Disord. 30, 584-9 (2015).

42. Pötter-Nerger, M., Reich, M. M., Colebatch, J. G., Deuschl, G. \& Volkmann, J. Differential effect of dopa and subthalamic stimulation on vestibular activity in Parkinson's disease. Mov. Disord. 27, 1268-75 (2012).

43. Seidel, K. et al. The brainstem pathologies of Parkinson's disease and dementia with Lewy bodies. Brain Pathol. 25, 121-35 (2015).

44. Dickson, D. W. Parkinson's disease and parkinsonism: neuropathology. Cold Spring Harb. Perspect. Med. 2, (2012).

45. Schirinzi, T. et al. Early synaptic dysfunction in Parkinson's disease: Insights from animal models. Mov. Disord. 0, 1-12 (2016).

46. Venhovens, J., Meulstee, J., Bloem, B. R. \& Verhagen, W. I. M. Neurovestibular dysfunction and falls in Parkinson's disease and atypical Parkinsonism: a prospective one year follow-up study. Artic. Submitt.

47. Bloem, B. R., Hausdorff, J. M., Visser, J. E. \& Giladi, N. Falls and freezing of gait in Parkinson's disease: A review of two interconnected, episodic phenomena. Mov. Disord. 19, 871-884 (2004).

48. Okuma, Y. Freezing of gait and falls in Parkinson's disease. J. Parkinsons. Dis. 4, 255-60 (2014).

49. Tomlinson, C. L. et al. Physiotherapy for Parkinson's disease: a comparison of techniques. Cochrane 
database Syst. Rev. CD002815 (2014). doi:10.1002/14651858.CD002815.pub2

50. Tomlinson, C. L. et al. Physiotherapy versus placebo or no intervention in Parkinson's disease. Cochrane database Syst. Rev. CD002817 (2013). doi:10.1002/14651858.CD002817.pub4

51. Tomlinson, C. et al. Physiotherapy intervention in Parkinson's disease: systematic review and metaanalysis. Bmj 345, e5004 (2012).

52. Allen, N. E., Sherrington, C., Paul, S. S. \& Canning, C. G. Balance and falls in Parkinson's disease: a meta-analysis of the effect of exercise and motor training. Mov. Disord. 26, 1605-15 (2011).

53. Keus, S. H. J., Munneke, M., Nijkrake, M. J., Kwakkel, G. \& Bloem, B. R. Physical therapy in Parkinson's disease: evolution and future challenges. Mov. Disord. 24, 1-14 (2009).

54. Radder, D. L. M. et al. Physical therapy and occupational therapy in Parkinson's disease. Int. J. Neurosci. 1-14 (2017). doi:10.1080/00207454.2016.1275617

55. Bloem, B. R. \& Munneke, M. Revolutionising management of chronic disease: the ParkinsonNet approach. BMJ 348, g1838-g1838 (2014).

56. de Vries, N. M., Sturkenboom, I. H. \& Bloem, B. R. Physiotherapy and Occupational Therapy and Mild to Moderate Parkinson Disease. JAMA Neurol. 73, 893-4 (2016).

57. Wilkinson, D., Podlewska, A. \& Sakel, M. A durable gain in motor and non-motor symptoms of Parkinson's Disease following repeated caloric vestibular stimulation: A single-case study. NeuroRehabilitation 38, 179-82 (2016).

58. Samoudi, G., Jivegård, M., Mulavara, A. P. \& Bergquist, F. Effects of Stochastic Vestibular Galvanic Stimulation and LDOPA on Balance and Motor Symptoms in Patients With Parkinson's Disease. Brain Stimul. 8, 474-80

59. Rizzo-Sierra, C. V, Gonzalez-Castaño, A. \& Leon-Sarmiento, F. E. Galvanic vestibular stimulation: a novel modulatory countermeasure for vestibular-associated movement disorders. Arq. Neuropsiquiatr. 72, 72-7 (2014).

60. Kataoka, H. et al. Can Postural Instability Respond to Galvanic Vestibular Stimulation in Patients with Parkinson's Disease? J. Mov. Disord. 9, 40-3 (2016).

61. Okada, Y. et al. Galvanic vestibular stimulation may improve anterior bending posture in Parkinson's disease. Neuroreport 26, 405-10 (2015).

62. Pal, S., Rosengren, S. M. \& Colebatch, J. G. Stochastic galvanic vestibular stimulation produces a small reduction in sway in Parkinson's disease. J. Vestib. Res. 19, 137-42 (2009). 

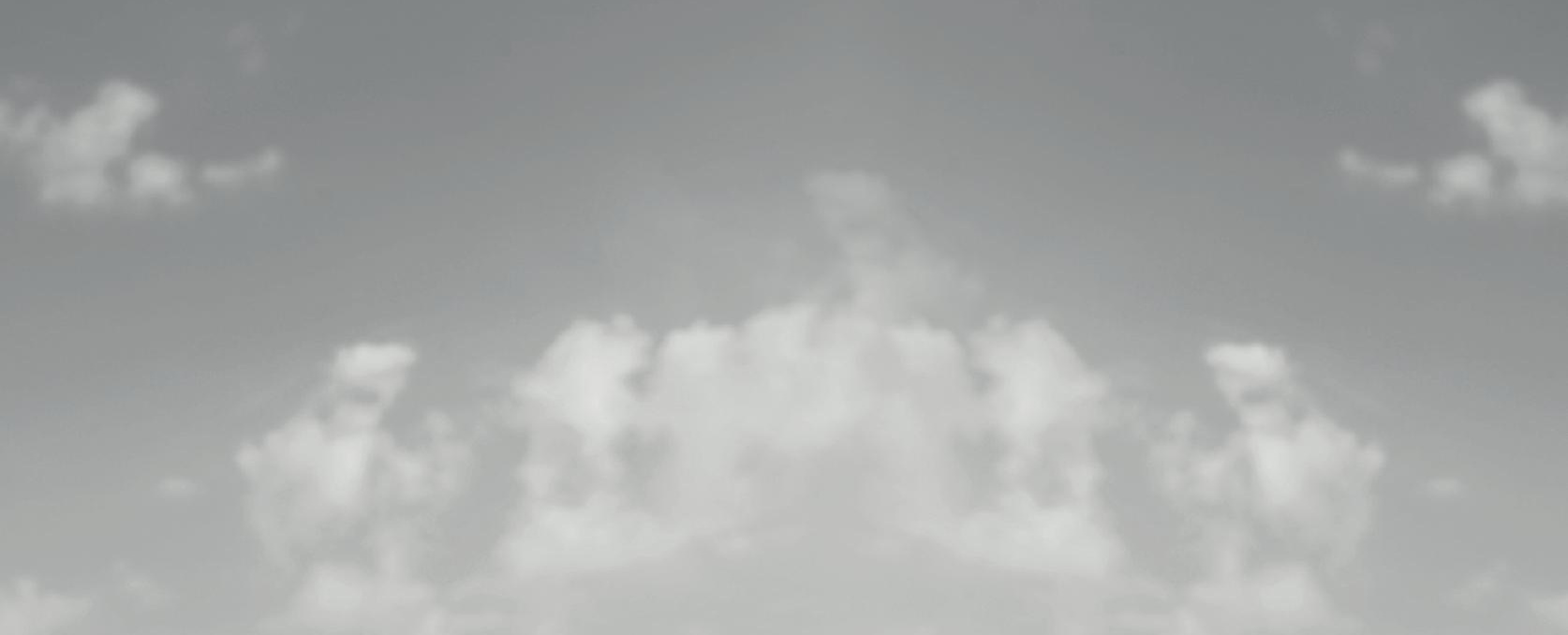

(1)
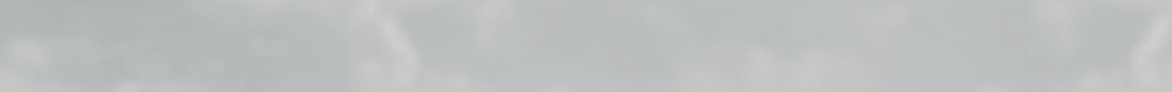

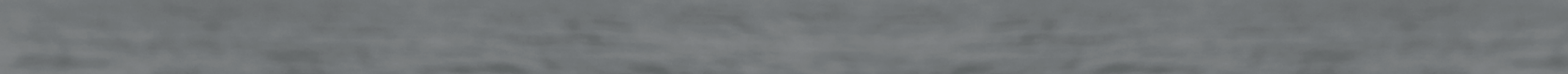

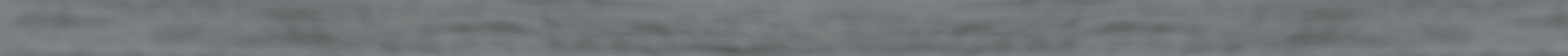
Wom

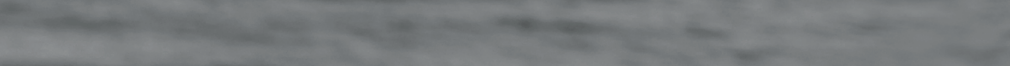
$=0$ 


\section{Appendices}

\section{List of abbreviations \\ List of publications \\ Dankwoord (Acknowledgements) \\ Curriculum Vitae}

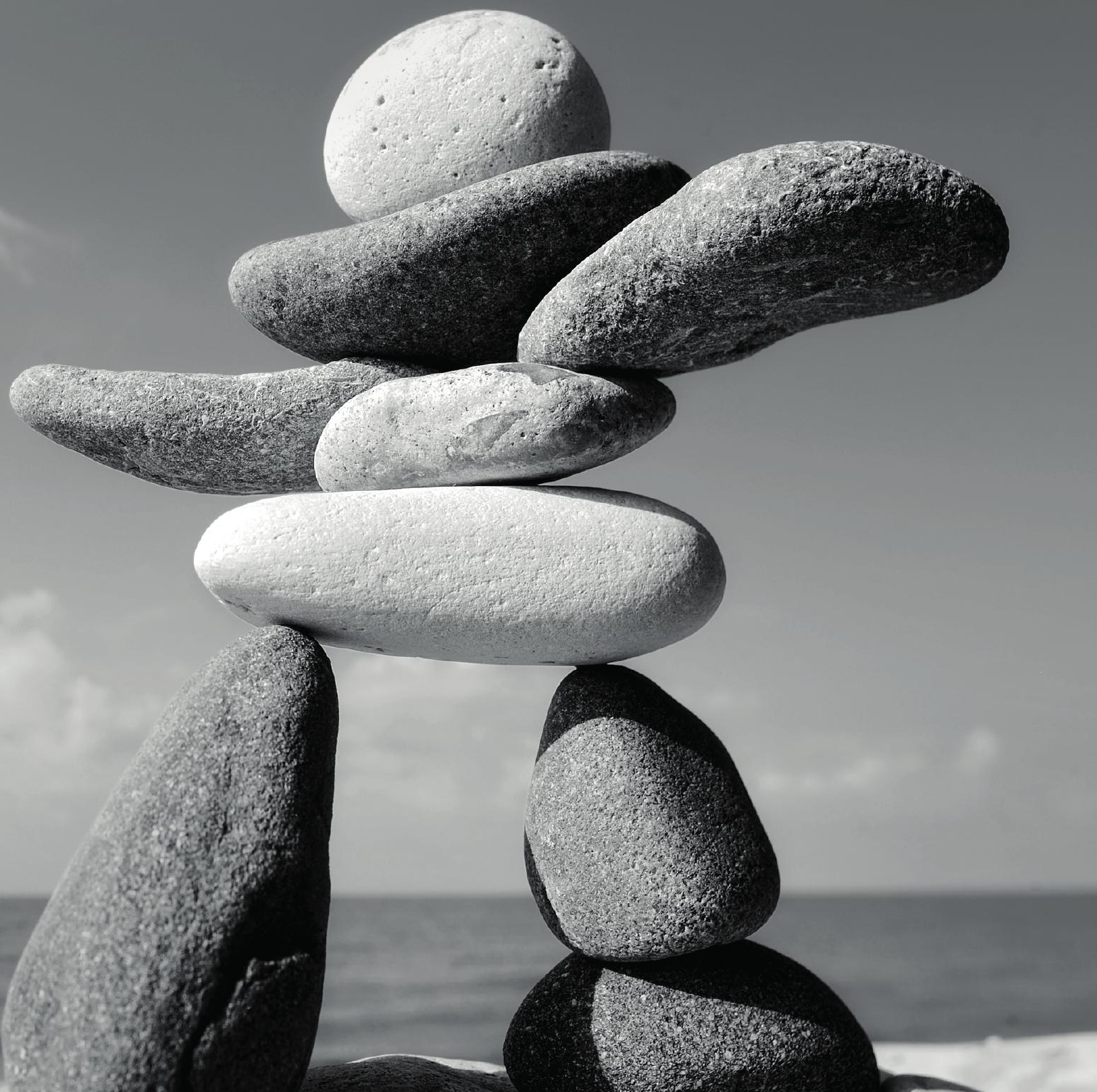




\section{List of abbreviations}

2AFC Two-alternative forced choice method

ABC-16 16 items activities-specific balance confidence scale

AC-cVEMP Air-conducted cervical vestibular evoked myogenic potentials

AC-oVEMP Air-conducted ocular vestibular evoked myogenic potentials

ADJ

ADL

AICA

Method of adjustment

AP

AVS

Activities of daily living

Anterior inferior cerebellar artery

BAEP

Atypical parkinsonism

Acute vestibular syndrome

BCV-cVEMP Bone-conducted cervical vestibular evoked myogenic potentials

BCV-oVEMP Bone-conducted ocular vestibular evoked myogenic potentials

CCA Cortical cerebellar atrophy

CT Computed tomography

cVEMP Cervical vestibular evoked myogenic potentials

dBNHL Decibels above normale adult hearing level

DHI Dizziness handicap inventory

DWI Diffusion weighted imaging

EDSS Expanded disability status scale

EMG Electromyography

ENG Electronystagmography

HINTS Head impulse, nystagmus, and skew deviation testing

ICC Intraclass correlation coefficient

ICHD-III International classification of headache disorders, 3th edition

INC Interstitial nucleus of Cajal

INO Internuclear ophthalmoplegia

LC Left cold

LW Left warm

$\mathrm{mcV} \quad$ Microvolts

MJD Machado-Jospeh disease

MLF Medial longitudinal fasciculus

$\mathrm{mmHg} \quad$ pressure in millimetres Mercury

MRI Magnetic resonance imaging

ms milliseconds

MS Multiple Sclerosis

MSA Multi system atrophy

MSA-p MSA with predominant parkinsonism 


$\begin{array}{ll}\text { OPCA } & \text { Olivo-ponto-cerebellar atrophy } \\ \text { oVEMP } & \text { Ocular vestibular evoked myogenic potentials } \\ \text { PD } & \text { Parkinson's disease } \\ \text { PICA } & \text { Posterior inferior cerebellar artery } \\ \text { PPV } & \text { Positive predictive value } \\ \text { PSP } & \text { Progressive supranuclear palsy } \\ \text { RC } & \text { Right cold } \\ \text { REM } & \text { Rapid eye-movement sleep } \\ \text { RW } & \text { Right warm } \\ \text { SCDS } & \text { Superior semicircular canal dehiscence syndrome } \\ \text { SCM } & \text { Sternocleidomastoid muscle } \\ \text { SHV } & \text { Subjective haptic vertical } \\ \text { SSCNS } & \text { Superficial siderosis of the central nervous system } \\ \text { SVV } & \text { Subjective visual vertical } \\ \text { ULN } & \text { Upper limit of normal } \\ \text { UPDRS } & \text { Unified Parkinson's disease rating scale } \\ \text { VEMP } & \text { Vestibular evoked myogenic potentials } \\ \text { VEP } & \text { Visual evoked potentials } \\ \text { VM } & \text { Vestibular migraine } \\ \text { VNG } & \text { Videonystagmography }\end{array}$




\section{List of publications}

1. Crevits, L., Venhovens, J., Vanoutrive, J. \& Debruyne, J. False perception of visual verticality in multiple sclerosis. Eur. J. Neurol. 14, 228-232 (2007).

2. Venhovens, J., Stelten, B., Feyen, B. F. E., van Dijk, G. \& Meulstee, J. Ischemic Stroke as a Complication of Varicella Zoster Encephalitis: A Case Report with Detailed EEG Discussion. Clin. EEG Neurosci. (2013).

3. Venhovens, J., Meulstee, J. \& Verhagen, W. I. M. The Negative Predictive Value of the Head Impulse Test, Nystagmus, and Test of Skew Deviation Bedside Oculomotor Examination in Acute Vestibular Syndrome. Ann. Emerg. Med. 66, 91-2 (2015).

4. Venhovens, J., Meulstee, J. \& Verhagen, W. I. M. Ocular and Cervical Vestibular Evoked Myogenic Potentials (VEMPs) in healthy volunteers: the intra-, interobserver, and the test re-test reliability. J. Vestib. Res. 25, 161-7 (2015).

5. Stelten, B. M., Venhovens, J., van der Velden, L. B., Meulstee, J. \& Verhagen, W. I. Syndrome of transient headache and neurological deficits with cerebrospinal fluid lymphocytosis (HaNDL): A case report with serial electroencephalography (EEG) recordings.. Is there an association with human herpes virus type 7 (HHV7) infection? Cephalalgia 36, 1296-1301 (2016).

6. Venhovens, J., Meulstee, J. \& Verhagen, W. I. M. Static Subjective Visual Vertical in Healthy Volunteers: The Effects of Different Preset Angle Deviations and TestRetest Variability. Neuroophthalmology. 40, 113-119 (2016).

7. Venhovens, J., Meulstee, J. \& Verhagen, W. I. M. Vestibular evoked myogenic potentials (VEMPs) in central neurological disorders. Clin. Neurophysiol. 127, 40-49 (2016).

8. Venhovens, J., Meulstee, J. \& Verhagen, W. I. M. Acute vestibular syndrome: a critical review and diagnostic algorithm concerning the clinical differentiation of peripheral versus central aetiologies in the emergency department. J. Neurol. 263, 2151-2157 (2016).

9. Venhovens, J., Meulstee, J., Bloem, B. R. \& Verhagen, W. I. M. Neurovestibular analysis and falls in Parkinson's disease and atypical parkinsonism. Eur. J. Neurosci. 43, 1636-46 (2016). 


\section{Dankwoord (Acknowledgements)}

$\mathrm{Na}$ een intensieve periode van ongeveer 6 jaar, waarbij ik mijn promotieonderzoek initieel heb uitgevoerd naast mijn medisch specialistische opleiding tot neuroloog (in het Canisius-Wilhelmina ziekenhuis, Nijmegen) en later naast mijn klinische werkzaamheden als neuroloog / klinisch neurofysioloog (in het Albert Schweitzer ziekenhuis, Dordrecht), is het uiteindelijk gekomen tot de laatste stap zijnde de openbare verdediging van dit proefschrift. Een wetenschappelijk onderzoek is nooit het werk van een individu, maar altijd het resultaat van een teamprestatie. Daarom zou ik graag van de gelegenheid gebruik willen maken om alle betrokkenen te bedanken.

Graag zou ik mijn dankwoord willen beginnen met het bedanken van alle patiënten, gezonde deelnemers en hun familieleden. Zonder uw inzet en bijdrage was het wetenschappelijk onderzoek, uiteindelijk leidend tot dit proefschrift, niet mogelijk geweest.

Professor dr. Bas Bloem (Radboud Universiteit, Nijmegen), graag wil ik je hartelijk bedanken dat je mijn promotor wilde zijn. Jouw gedrevenheid, enthousiasme en creatieve out-of-the-box ideeën waardeer ik zeer. Dr. Wim Verhagen en dr. Jan Meulstee (Canisius-Wilhelmina ziekenhuis, Nijmegen), jullie waren als copromotoren mijn directe begeleiders en daardoor de drijvende kracht achter dit proefschrift. Altijd to-the-point, kritisch, constructief, secuur en zeer snel in het terugsturen van jullie commentaar, altijd leidend tot een duidelijke verbetering van de manuscripten. Hierbij kon ik me geen betere begeleiding wensen. Beste Wim, naast jouw begeleiding als copromotor wil ik je ook heel erg bedanken voor jouw goed vervulde rol als opleider. Jouw recente verkiezing tot opleider van het jaar is dan ook niets minder dan verdiend. Naast de overdracht van algemeen neurologische en met name neurovestibulaire medische kennis, heb je als rolmodel mij ook duidelijk ondersteund in mijn persoonlijke ontwikkeling tot medisch specialist. Tot op heden profiteer ik hier nog dagelijks van en kijk ik met een zeer goed gevoel terug op mijn opleiding tot medisch specialist. Beste Jan, dank voor het delen van jouw uitgebreide klinisch neurofysiologische kennis. Deze gedegen kennis maakt me niet alleen een betere klinisch neurofysioloog, maar ik ben er tevens van overtuigd dat dit me ook een betere klinisch neuroloog maakt. Naast het medisch inhoudelijke deel van het vak kijk ik ook met een goed gevoel terug op zowel onze persoonlijke gesprekken alsmede op de gesprekken over onze gedeelde hobbie, zijnde de astrofysica. Na het afronden van dit promotieonderzoek kom ik graag een keer langs om jouw observatorium te komen bewonderen en onze gesprekken voort te zetten. 
Zonder de ondersteuning van de laboranten en secretaresse van de afdeling klinische neurofysiologie uit het Canisius-Wilhelmina ziekenhuis te Nijmegen was dit onderzoek eveneens niet mogelijk geweest. Dank jullie voor de praktische ondersteuning, constructieve blik op de technische uitvoering van het onderzoek, maar vooral ook voor de aangename en erg gezellige samenwerking.

De manuscriptcommissie bestaande uit professor dr. Henri Marres (Radboud Universiteit Nijmegen), professor dr. Jelte Bos (Vrije Universiteit, Amsterdam) en professor dr. Sander Geurts (Radboud Universiteit, Nijmegen) wil ik hartelijk bedanken voor de bereidheid dat ik u mijn proefschrift ter beoordeling mocht voorleggen. Naast de manuscriptcommissie zou ik graag professor emeritus dr. Luc Crevits (Universiteit Gent, België), professor dr. Floris Wuyts (Universiteit Antwerpen, België), professor dr. Henk Berendse (Vrije Universiteit, Amsterdam) en dr. Bart van de Warrenburg (Radboud Universiteit, Nijmegen) als overige leden van de promotiecommissie (naast de leden eerder vermeld in de manuscriptcommissie) bij voorbaat willen bedanken voor hun kritische beschouwing van mijn proefschrift. In het bijzonder zou ik hierbij zeer graag professor emeritus dr. Luc Crevits willen bedanken. Beste Luc, de basis van dit proefschrift en mijn neurovestibulaire interesse werden beide al zeer vroeg tijdens mijn opleiding geneeskunde bij u gelegd. Graag wil ik u nogmaals zeer hartelijk bedanken voor de mogelijkheden die u me heeft geboden om me zowel klinisch medisch als wetenschappelijk te ontwikkelen op uw afdeling neurologie aan de Universiteit Gent. Vooral uw scherpe kritische blik, oog voor details, zeer uitgebreide klinisch neurologische kennis, maar voornamelijk ook uw zeer vriendelijke en rustige bejegening waren en zijn voor mij een voorbeeld. Graag zou ik direct van de gelegenheid gebruik willen maken om alle stafleden, (oud) arts-assistenten, verpleegkundigen, secretaresses en het ondersteunend personeel van de afdeling neurologie uit het Canisius-Wilhelmina ziekenhuis te Nijmegen willen bedanken voor de samenwerking, collegialiteit en zeer goede sfeer tijdens mijn opleiding tot neuroloog.

Mijn collega neurologen uit het Albert Schweitzer ziekenhuis, waar ik tegenwoordig werkzaam ben, wil ik graag bedanken voor hun collegialiteit, vriendelijkheid, vertrouwen en geduld. We hebben samen een drukke praktijk, maar omdat we allen tezamen onze schouders eronder zetten, maken vele handen het werk een stuk lichter en leuker. Ik kijk uit naar onze langdurige samenwerking, waarbij er naast het medisch inhoudelijke aspect, gelukkig ook aandacht is voor humor en de persoon achter de dokter. Dank jullie voor de fijne samenwerking. Naast natuurlijk de neurologen dragen de physician assistants, arts-assistenten, doktersassistenten, KNF-laboranten, secretaresses, verzorgenden en verpleegkundigen in belangrijke mate bij aan de fijne 
werkomgeving. Dankzij jullie ga ik iedere dag met een glimlach aan het werk waarvoor heel hartelijk bedankt.

Beste Mischa en Monique Vreeburg, jullie steun als paranimfen betekent veel voor me. Sinds we elkaar kennen, vanaf onze studie scheikunde aan de Universiteit Utrecht, beschouw ik onze vriendschap als zeer hecht en waardevol. Zowel in goede tijden als in tijden wanneer het even tegenzat, kon ik altijd op jullie bouwen.

Buiten het medische en het wetenschappelijk vlak zijn er natuurlijk ook veel mensen, die in zeer belangrijke mate hebben bijgedragen aan de voltooiing van dit proefschrift. Als eerste zou ik heel graag mijn vrouw Mariska Venhovens-Janssen willen bedanken voor alle fijne liefdevolle jaren samen en ook voor het op de wereld brengen van onze dochter Nienke en onze aankomende dochter, die binnenkort zal worden geboren. Zonder jouw steun was dit proefschrift er nooit geweest en was mijn leven een stuk minder compleet. Daarnaast wil ik ook heel erg graag mijn (schoon)ouders, (schoon) familie en al mijn lieve vrienden bedanken voor hun onvoorwaardelijke steun. 


\section{Curriculum Vitae}

Jeroen Venhovens werd op 14 maart 1981 geboren in Gorinchem. In 2000 behaalde hij zijn VWO diploma aan de Christelijke scholengemeenschap de Oude Hoven te Gorinchem. Vanwege de numerus fixus op de opleiding geneeskunde, heeft hij in de periode 2000-2001 de studie scheikunde aan de Universiteit Utrecht gevolgd als vooropleiding, waarbij het propaedeutisch examen met succes werd afgerond. In het daaropvolgende studiejaar is hij vanwege de numerus fixus uitgeweken naar de Universiteit Gent te België om aldaar de studie geneeskunde aan te vangen. Gedurende de studie geneeskunde groeide zijn interesse voor de neurologie (en met name de neurovestibulaire problematiek) door de verschillende wetenschappelijke en klinische stages bij de afdeling neurologie van het Universitair ziekenhuis Gent te België onder begeleiding van professor dr. Luc Crevits. Na het succesvol behalen van zijn master diploma geneeskunde aan de Universiteit Gent te België in 2008 (afstudeerrichting ziekenhuisarts - wetenschappelijk onderzoek), begon hij direct aansluitend met zijn werkzaamheden als arts-assistent neurologie niet in opleiding (ANIOS) in het Jeroen Bosch ziekenhuis te's Hertogenbosch tot medio 2009. Eind 2009 begon hij met de opleiding tot neuroloog in het Canisius-Wilhelmina ziekenhuis te Nijmegen (opleider dr. Wim Verhagen). Het wetenschappelijk onderzoek wat uiteindelijke leidde tot dit proefschrift begon hij in 2012 en werd met name uitgevoerd tijdens zijn verdere verdieping in de klinische neurofysiologie in 2014 en 2015 (opleider dr. Jan Meulstee). $\mathrm{Na}$ het afronden van zijn opleiding tot neuroloog in 2015, is hij vanaf die tijd werkzaam in het Albert Schweitzer ziekenhuis te Dordrecht als neuroloog met de aandachtsgebieden klinische neurofysiologie en neurovestibulaire stoornissen. 

